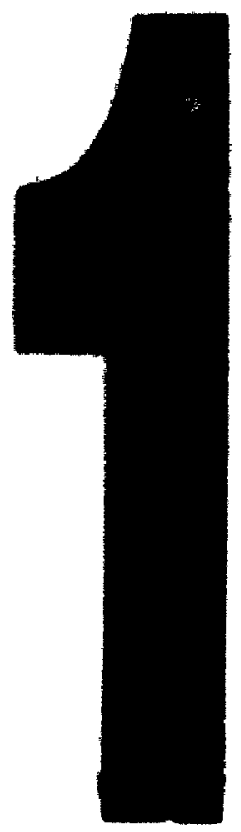

PM-1 31/2" "4" PHOTOCRAPHC MICAOCOPY TARCET nes nove AMsh/SO 2 EOUIVALENT

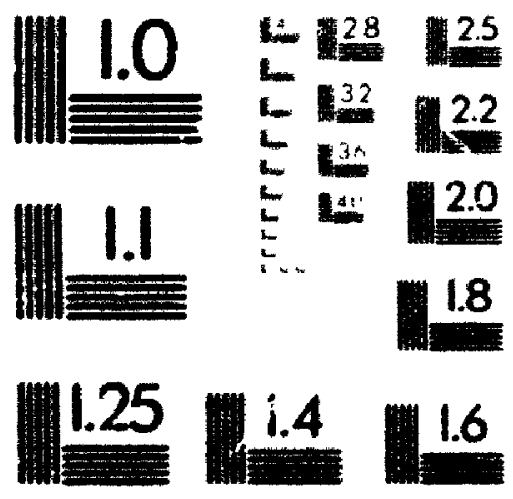

preciosonem nesoumion tancets 


\title{
Meson Properties in the Quark Model: A Look at Some Outstanding Problems
}

\author{
b.
}

\author{
Harry G. Blundell, B.Sc. \\ A thesis submitled io \\ the Faculty of Ciraduate Studien anc Research \\ in partial fultilment of \\ the reciuirements for the degrere of \\ Doctor of Phikesephy \\ Ottawa-Carleton Inctitute for Physics \\ Department of Physics \\ Carleton University \\ Ottaw. Ontario. Canada \\ July 19. 199\%, \\ CHarry (i. Blundell. I996;
}


National Library

of Canada

Acquisitions and

Bibliographic Services Branch

395 Wethrington Street

Ontawa Onlano

KIA ONA
Bibiolheque nationale

du Canada

Direction des acquisitions ef des services bibliographiques

395, rue Wellington

Ottawa (Ontario)
The author has granted an irrevocable non-exclusive licence allowing the National Library of Canada to reproduce, loan, distribute or sell copies of his/her thesis by any means and in any form or format, making this thesis avallable to interested persons.

\begin{abstract}
L'auteur a accordé une licence irrévocable et non exclusive permettant à la Bibliothèque nationale du Canada de reproduire, prêter, distributer ou vendre des copies de sa thèse de quelque manière et sous quelque forme que ce soit pour mettre des exemplaires de cette thèse à la disposition des personnes intéressées.
\end{abstract}

The author retains ownership of the copyright in his/her thesis. Nelther the thesis nor substantial extracts from it may be printed or otherwise reproduced without his/her permission.
L'auteur conserve la propriété du droit d'auteur qui protege sa thèse. Ni la these ni des extraits substantiels de celle-ci ne doivent être imprimés ou autrement reproduits sans son autorisation.

ISBN C 512-19357-8 
Nome HARRY BLUNDELL.

Dissertation Abstracts International is arrany ud by brood, general subject calogories. Please select the one subject which most neorly describes the content of your dissentotion Enter the corresponding four-digit code in the spaces provided

\section{ELEMENTARY PARTICLES AND HIEN ENERGYOT7918 U.M.I

\section{Subiect Colvopries}

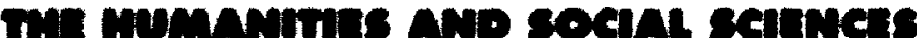

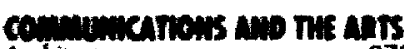

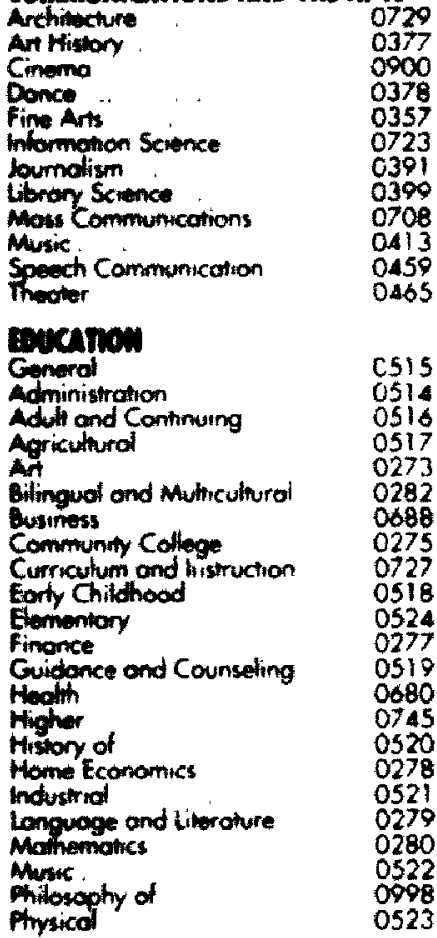

Psychotogy

Reoding

Reingrous

Scrences

Secondary

Soctal Sciences

Saciolagy of

Special

Teacher Troining

Technology

0,710

Tests and Meosurements $\quad 0288$

Vocotiona?

Ineunet, umathat ans uncatrics

Languoge

$\begin{array}{ll}\text { Einquage } & 067 \\ \text { Ancient } & 028 \\ \text { Lingustics } & 029 \\ \text { Madern } & 029\end{array}$

Moder

Genero

Clossucol

Comparativo

Alediewal

Modern

Afreon

Americon

Asion

Conodian (English)

Conodian (French)

English

Germonic

Luth Americon

Middle Eostern

Romonce

Stovic and Fost Eutopean

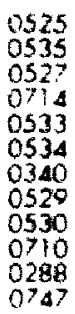

0679

0290

0401

0294

0295

0297

0208

0310

0591

0.305

0352

0355

0593

0311

$\mathrm{C}+12$

0315

0313 monosowr, ntuenow ans

mitoras

Philosoptiv

Relghon

Gernesal

Biblical Studies

Chetgy

History of

Phitosopty of

Theology

\section{soan scitnces}

American Studies

Antropology

Archomolrox

Cultura

Physicat

Busines, Administrofion

Genero

Acrounting

Bonking

Monogement

Marketing

Conodian Studie:

Eroncmiss

General

Aquiruhural

Commerre

Finance

History

theory

Foltotor

Geogrophy

Garoritriog

Aistory

brement?

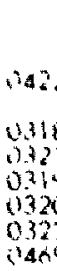

0,318

वर्ष

1320

0325
$\sin$

13,129

0324

6)

(4) 390

427

$0+7$

1454

is

$63 \mathrm{H}^{\circ}$

6501

asins

osust

Gros

$0 \times 19$

(15) 16

बiा

9356

()) $16 \mathrm{se}$

$\operatorname{lis} 7 \mathrm{~B}$

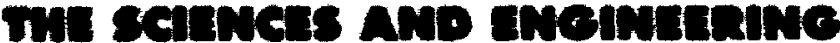

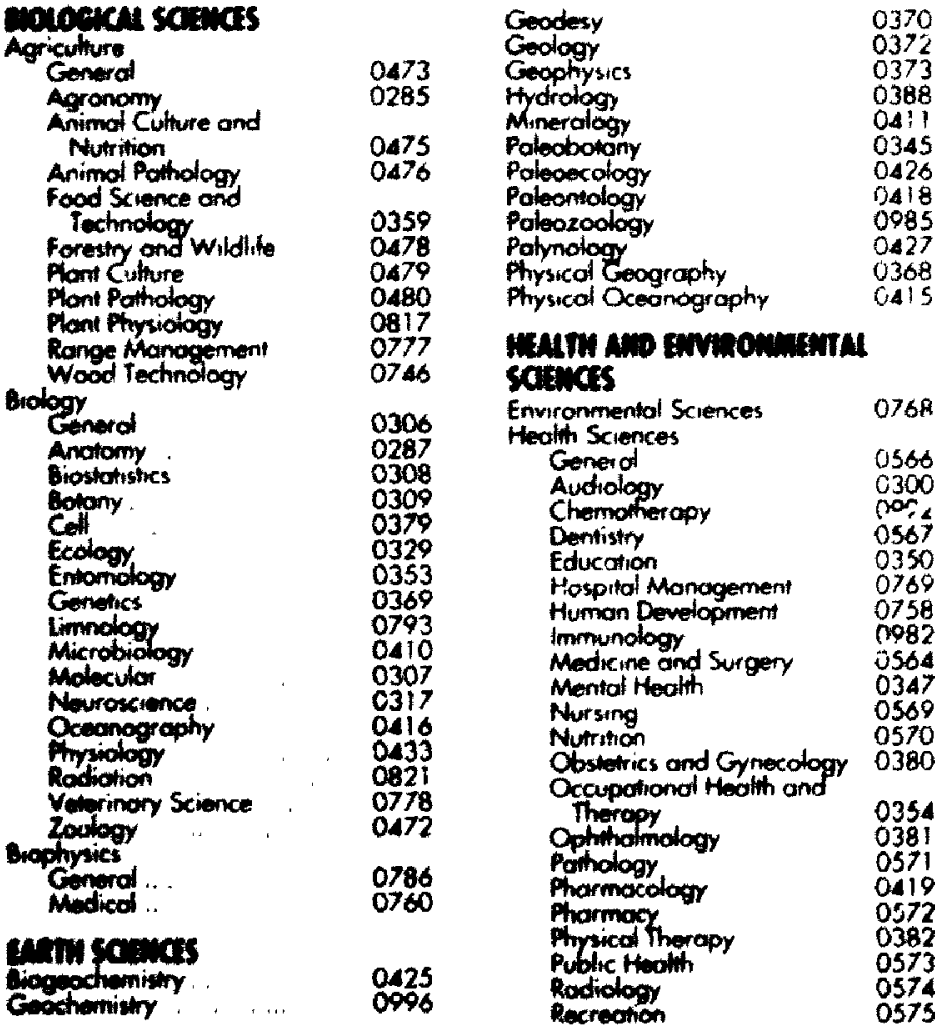

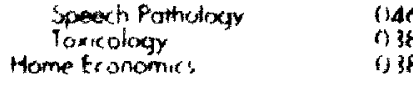

\section{mistcul somas}

Pure Sciences

Chemistry

Genero:

Agrecutisers

Broxhemistry

inorganic

Pvicteor

Organir

Phermareuterts

Physur at

Polvonion

Mathernatics

Physirs

Conerol

Astronomy and

Astronomy and

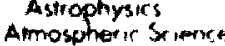

Alomic

Electronks and E ist miectity

Elementary Porticies and

High Energy

Fluor ind Plasmo

Matecular

Nuxleor

Optics

Rodiction

Solud Sicie

Stotistics

Applind sciences

Applied Mechemes

Computer Scrence
() $36(4)$

G) BBe

(2) 485

0748

(1)485

0,487

$04 \mathrm{~KB}$

07 is

(1) 140

$04 \% 1$

0494

$04{ }^{\circ}$

(1)734

0465

usins

orpses

$\cos 50$

OSOSO

$0 / 48$

Das?

0798

075

$060 \%$

0610

075

QSiI

0463

0345
Anuent:

Mardereys

Miniters:"

Atow

Alitum

Asio Austo

Carkidit

cisten Ament on

Modithe tristern

itrinel sum:

thestion w werter

taw

Proluted at ivereme

(a)remist

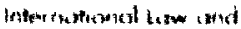

Ratations

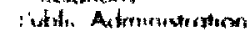

Remingines?

"xx toil W.."

in w thlixgy

I. The is?

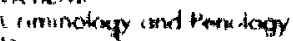

Chetmerritionty

Ethren and Row iol Stentmes

indiventerl and lomity

Sturties

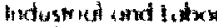

Reikntionts

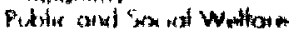

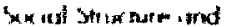

Devef.. yomerit

Theowy and Methaxh.

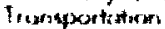

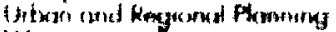

Wremers sitturlies:

$\sin \theta$

MBs

ASB 2

a.t!

a.134

0,334

atis

033

(1) 38 :

mats

oblo

si.

O45?

00.0

1602

$1 x+1$

onde

1620

$\tan x$

n7wo

0344

oroy

0.53)

Pregunation

Ateriseyst

Aggrse whitor

Autrenistives

atremers

ivil

fleotiones and chertiral

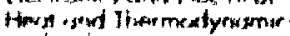

Ayetrous:

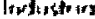

Mkstitio

Mesamests "n inere

Mesturain on

Metrithivey

Minsines

the best

iratexpiry

Petrosteriyn

condesty omd Mylut apes

'systert: "or senre

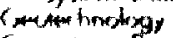

cperenton is Renwerh

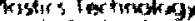

extite intirnotsy

FrTamesy

cences

(lience)

Davehaponation

fopmitruerters 
The nuderigned reesmmend to

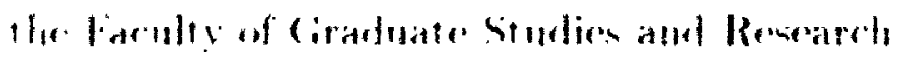
aenertaner of the thesis

\section{Meson Properties in the Quark Model:}

\section{A Look at Some Outstanding Problems}

shmined hy Harry G. Blundell, B.Sc.

in partial fulfiment of the recuirement o for

the degree of I Docter of Phit sophy

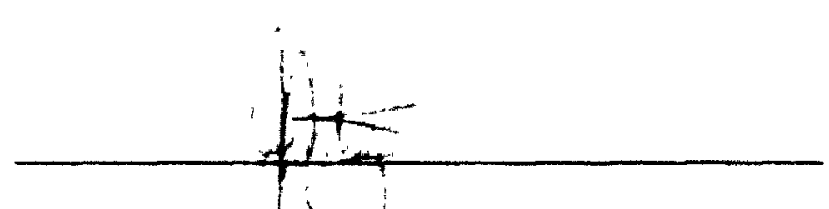

Chair, Bepertment of Physics

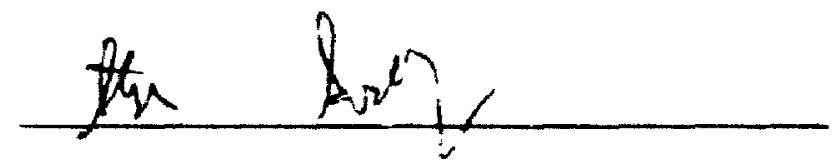

Thesis Supervisor

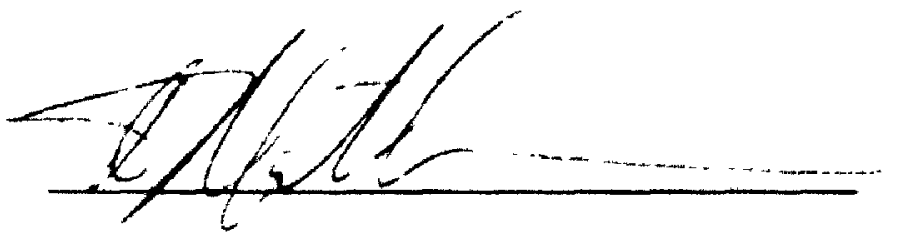

External Examiner

Carkon Iniversity

Dat" Angust $25,19: 6$ 


\section{Abstract}

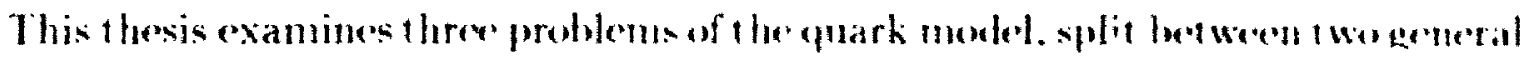
areas.

The first area involves mocels of meson decal. I he calculations of dende widehs in two such models. the "II moded and the flus-tube breaking model. were antomated so that they could be easily done for any decay. Mle modeds wee then wed wo

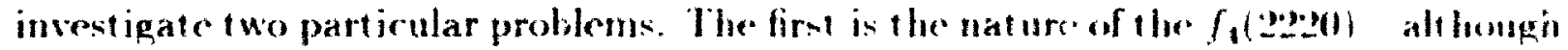

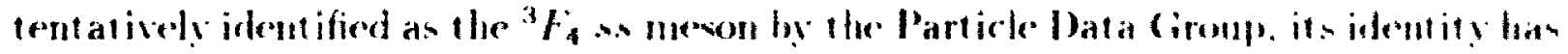

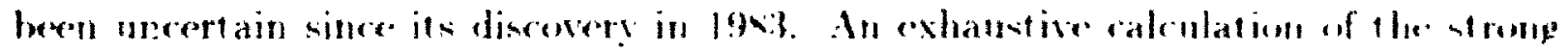

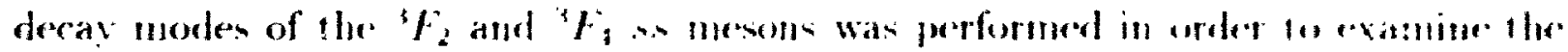

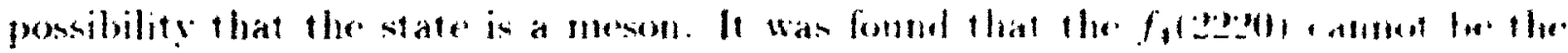

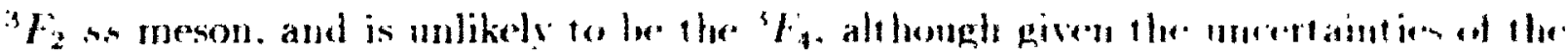

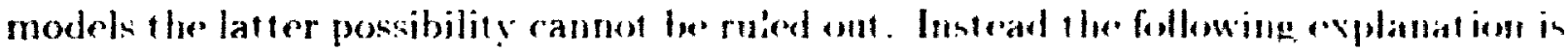

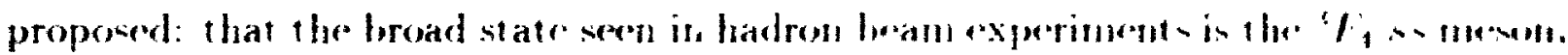

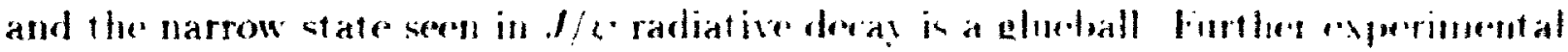
data is needed to tinally idemtify the $f, 1222021)$

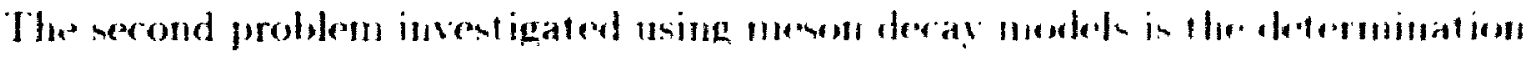

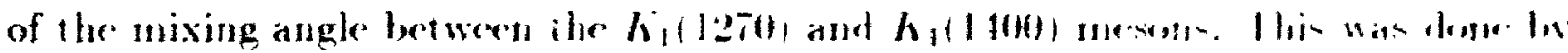

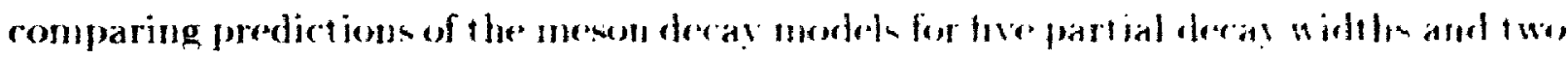
ratios of D to Samplitudes to experimentaldata. A miximg ande of appleximatels $1 f^{\prime}$

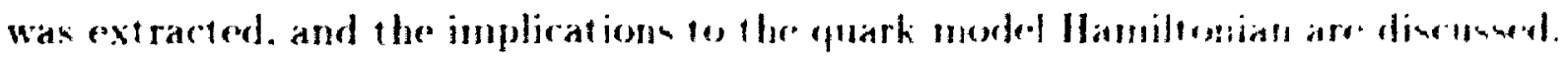

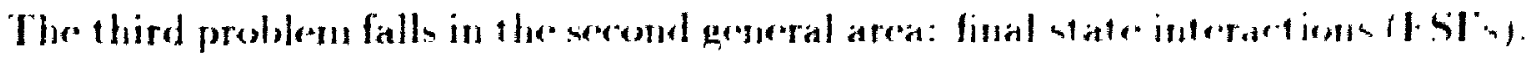

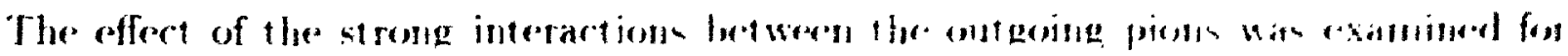

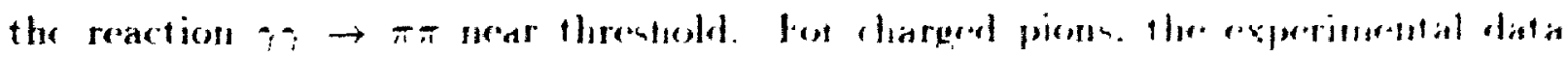

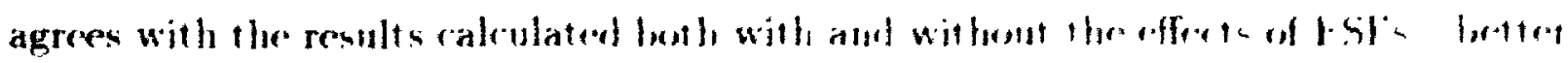




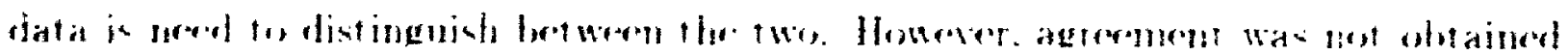

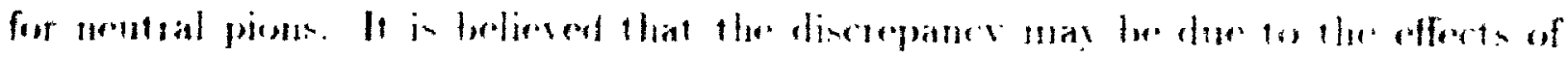

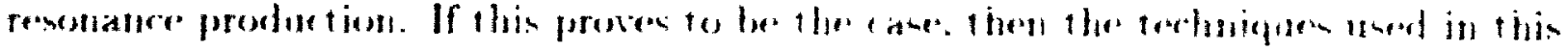

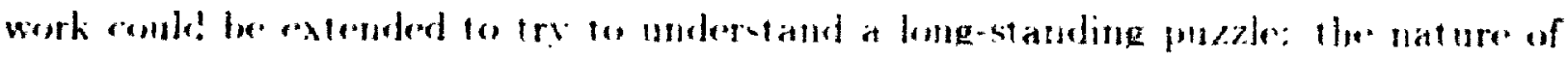

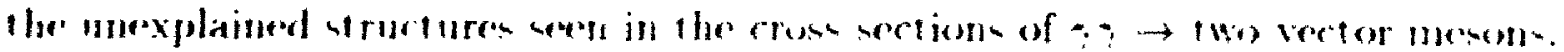




\section{Acknowledgments}

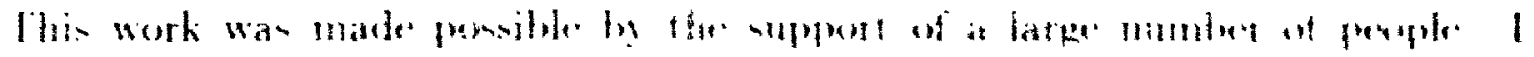

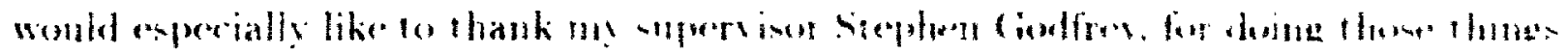

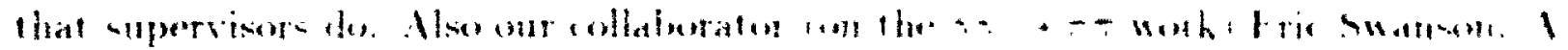

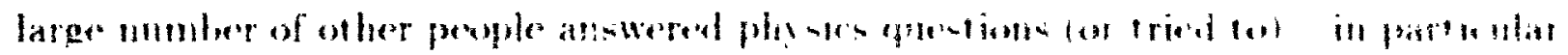

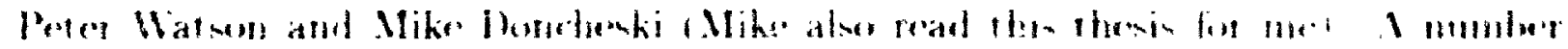

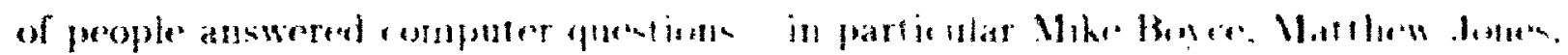

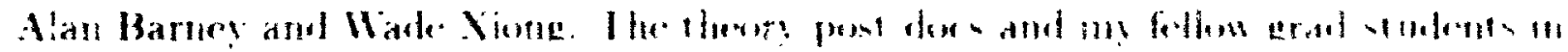

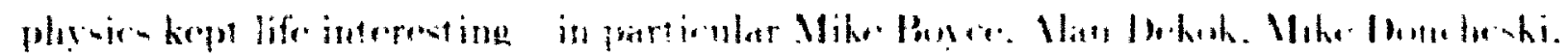

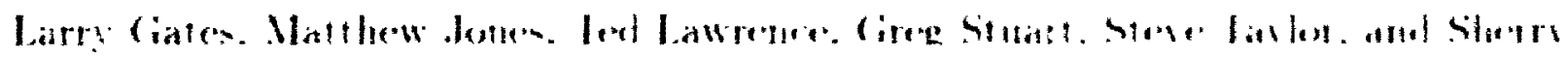

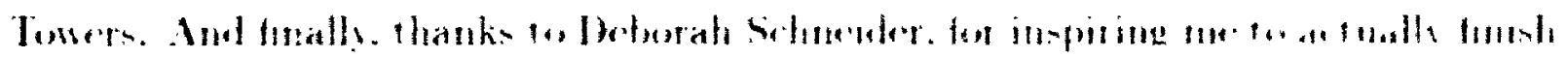




\section{Contents}

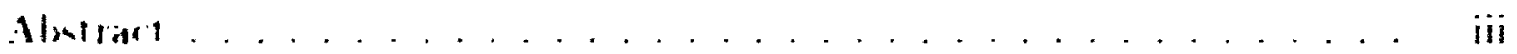

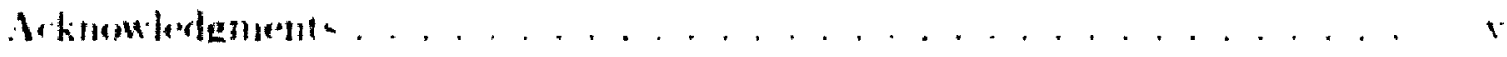

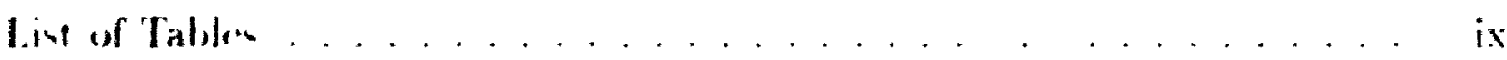

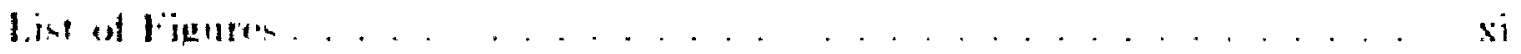

1 Introduction 1

1.1 The Standard Model of Particle Phycir . . . . . . . . . . . 2

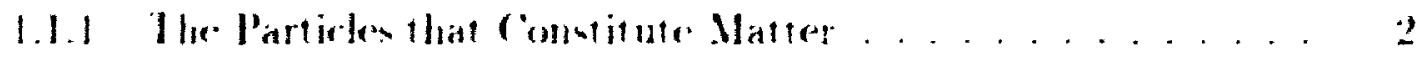

1.1.2 The Interartion ................. 1

l.I The Hadrons .......................

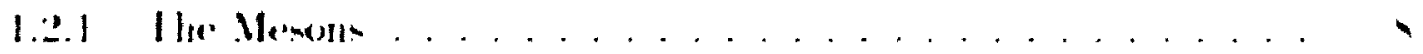

1.2.2 Th. Quark Modet . . . . . . . . . . . . . . . . . 4

l.8 The llork of this The . . . . . . . . . . . . . . . . . 12

2 Two Models of Meson Decay 15

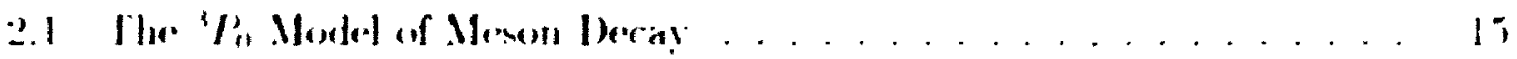

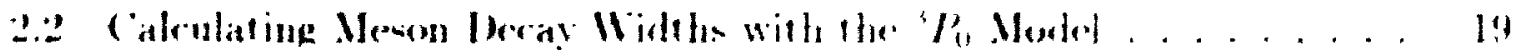

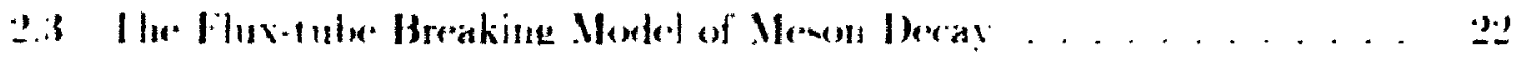

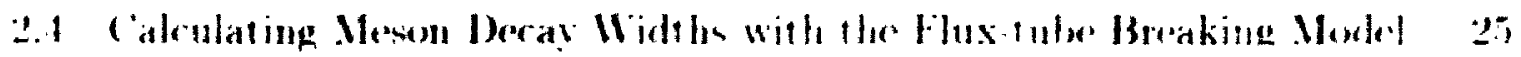

3 Models of Meson Decay: Two Applications 31 


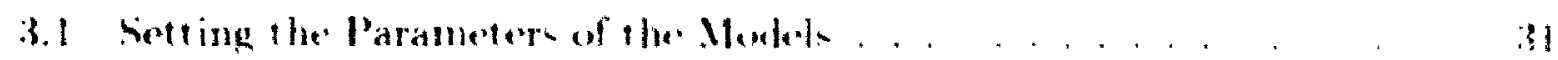

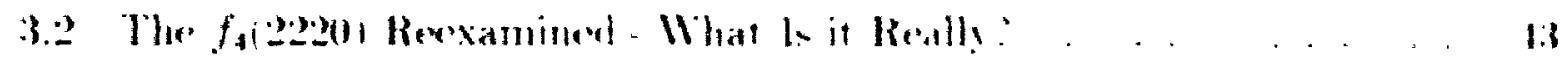

3.3 Comstraining the $h$ i Mixing dngk . . . . . . . . . . . . . . .

4 Effects of Final State Interactions

1.1 Introduction to Final state Interaction-............ toll

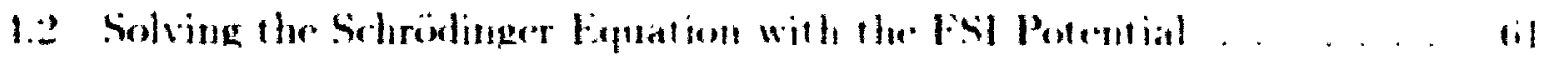

1.3 The Fermi Approximation . . . . . . . . . . . . . . . . . .3

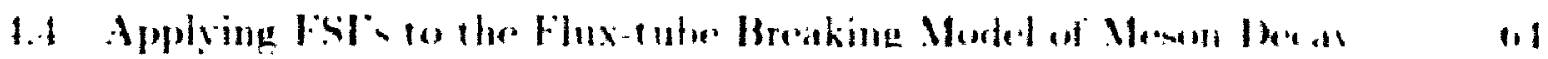

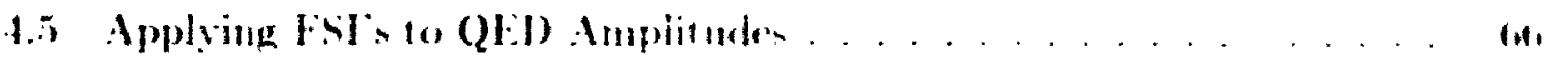

5 Final State Interactions: An Application

5.1 The Interaction $\ldots \rightarrow-. \ldots \ldots \ldots$

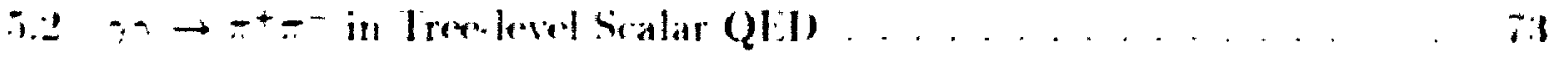

j.3 Applying the fist corrertions . . . . . . . . . . . . . . . . iti

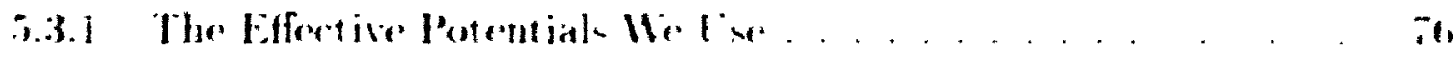

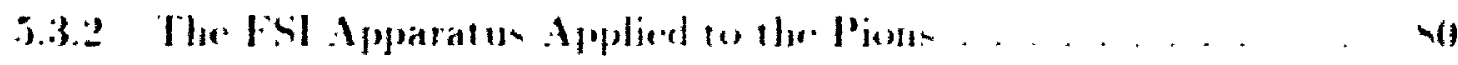

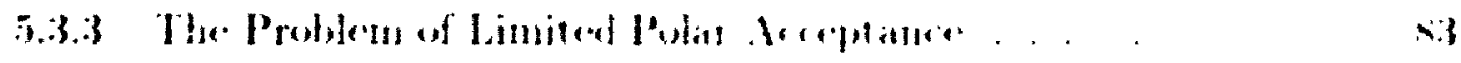

$\$ .3 .1$ Ninmerical betail. . . . . . . . . . . . . .

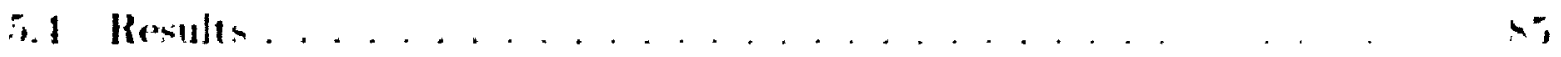

6 Conclusions $\quad 93$

A Some Tools of Particle Physics M;

1.1 Crom-section and llidth . . . . . . . . . . . . "1,

1.2 Fegnman Diagrams. . . . . . . . . . . . . . . . . . . 17

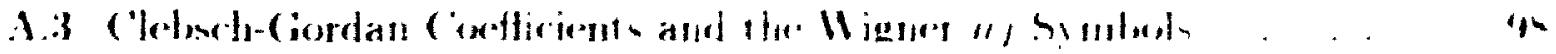

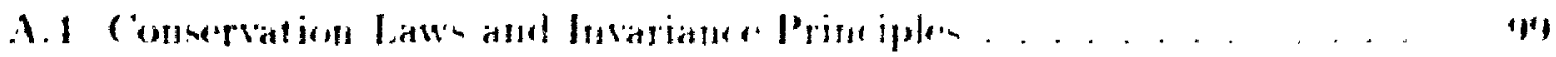

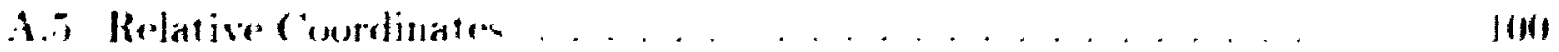




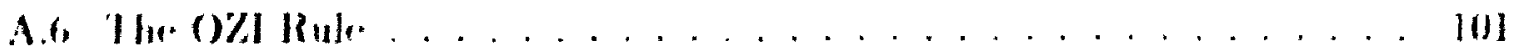

A.t Menon Mixings . . . . . . . . . . . . . . . . 101

B Meson Wavefunctions Ised in this Work 104

B.1 Sipare llincefunctions . . . . . . . . . . . . . . . . 101

B.: Flasour Wavefunctions . . . . . . . . . . . . IOti

C Field Theory Conventions Lised in this Work 108

D Evaluating the Calour. Flavour and Spin Overlaps for Models of Meson Decay 110

10.1 Colom Overlap . . . . . . . . . . . . . . . . 110

D.: Hanour Overlap . . . . . . . . . . . . . . . IIZ

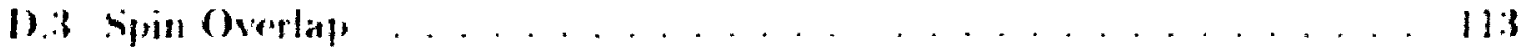

E Converting to Partial Wave Amplitudes for Models of Meson Decay $\quad 116$

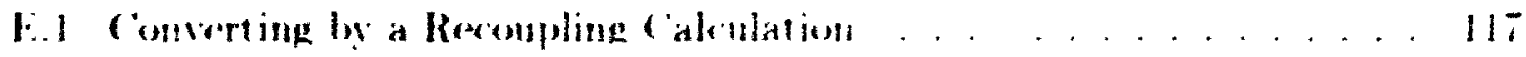

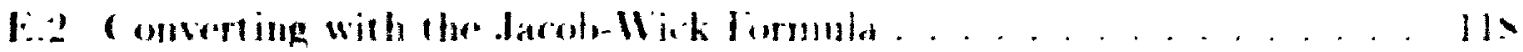

$\begin{array}{ll}\text { Bibliography } & 119\end{array}$ 


\section{List of Tables}

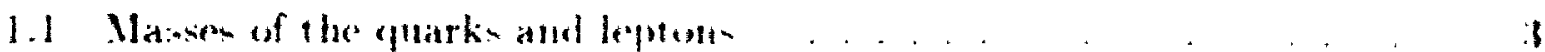

1.2 The known memon spectrum for the likh quark-. . . . . . II

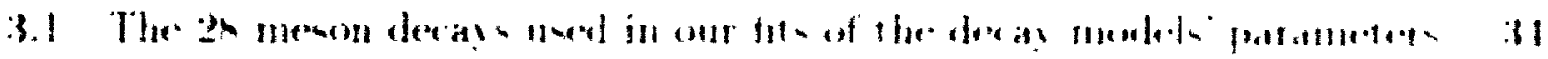

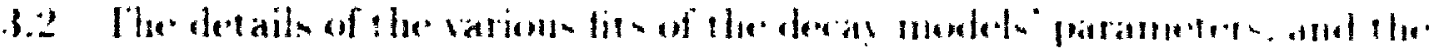

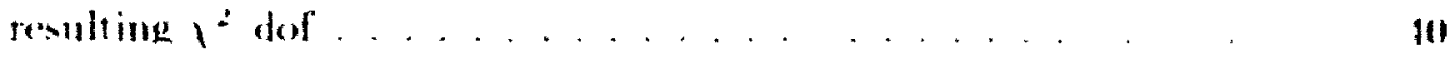

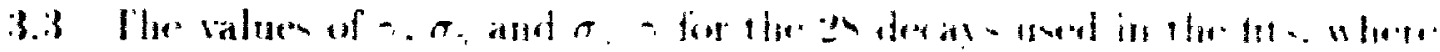

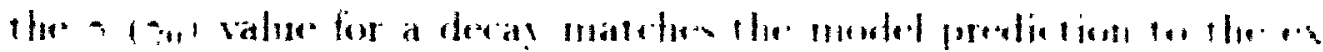

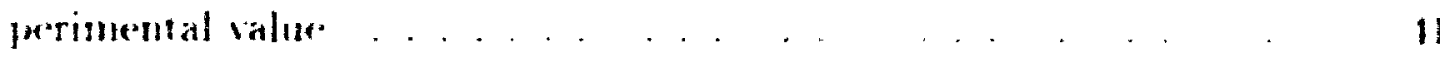

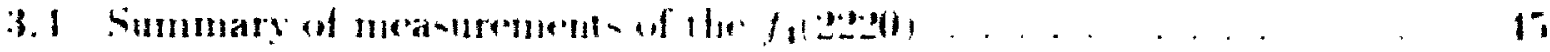

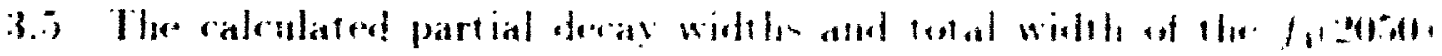

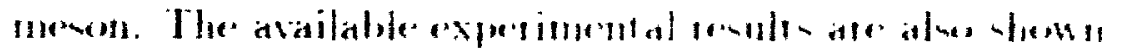

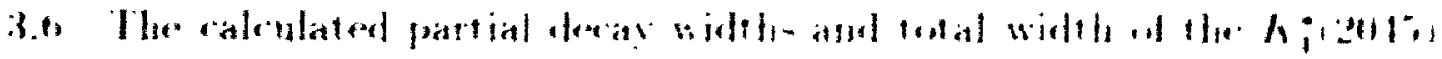

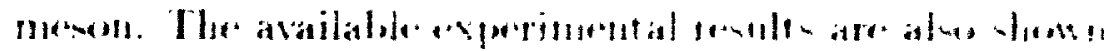

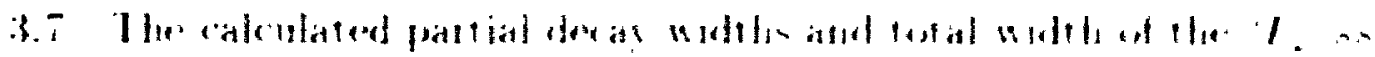
mentil

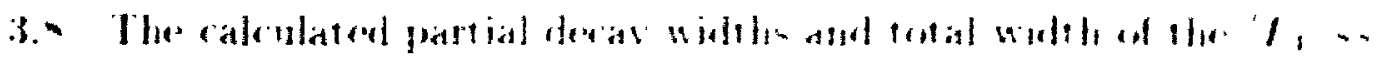
messhl . . . . . . . . . . .

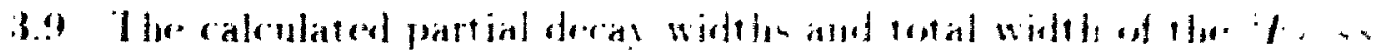
III $3 \times(3)$ 


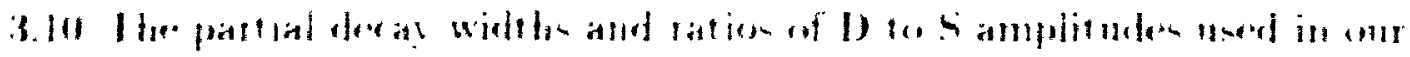

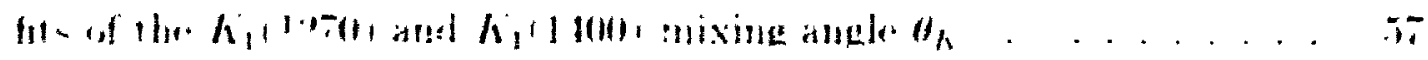

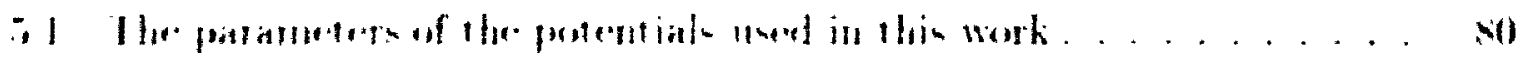




\section{List of Figures}

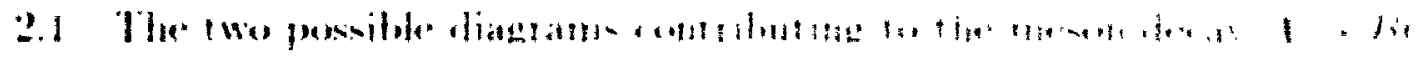

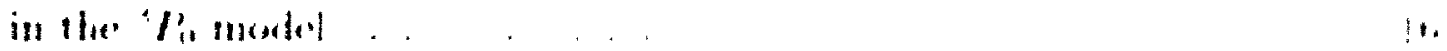

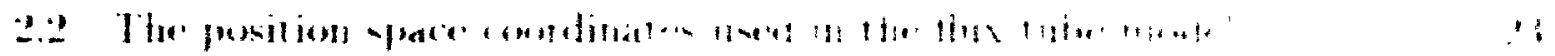

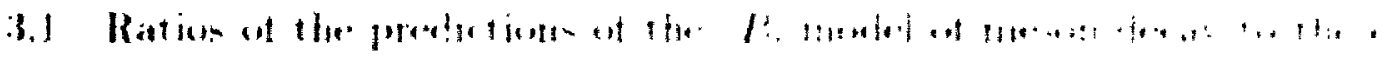

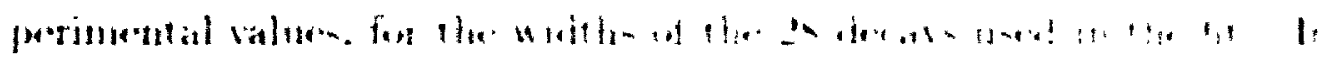

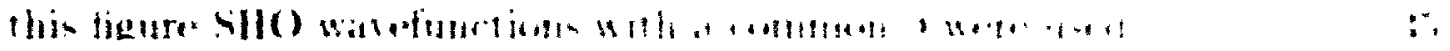

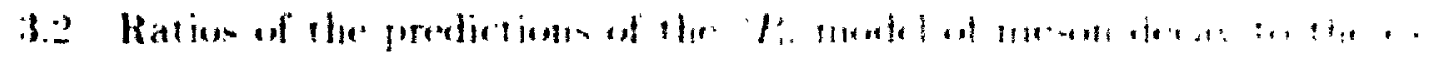

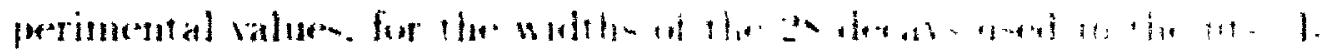

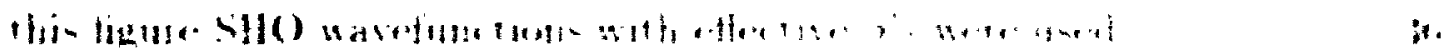

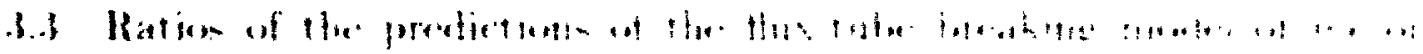

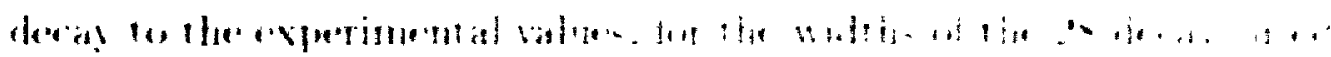

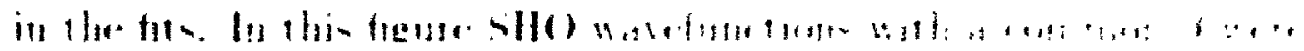
iared

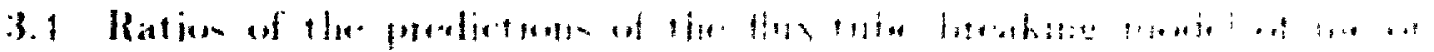

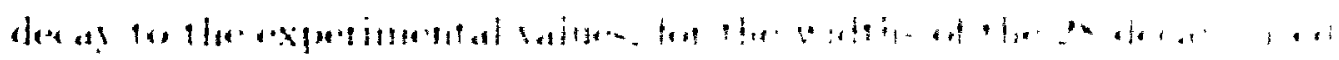

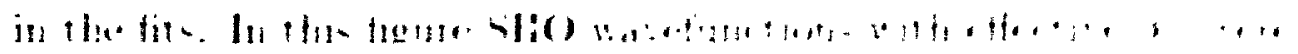
unsed 
3.5 Ratios of the predictions of the flux-tube breaking model of meson deray to the experimental values. for the widths of the 28 decays used in the fits, In this figure RQM wavefunctions were used . . . . .

3.6 Ratios of the predictions of the ${ }^{3} P_{0}$ model of meson decay to the experimental values. for the widths of the 28 decays used in the fits. In this figure SHO wavefunctions with o common 3 were used. but both I and $y$ were fit simultaneously ..................

3.7 The oniginal dat a from the discovery of the $f_{4}(2220)$ by the MARK III Collaboration. The dist ribution of the $h^{+} K^{-}$invariant mass in $\mathrm{C}^{+}+$ $J / 4^{\circ} \rightarrow \gamma h^{+} h^{-}$is shown. reveaiing three risonances . . . . . . .

3.8 (iraphs of the $h_{1}(1270)$ partial decay widths and ratio of $D$ to $S$ amplitudes considered in this work. vs. the mixing angle $\theta_{k} \ldots$. . . . .

3.9 Graphs of the $h_{1}(1400)$ partial decay widths and ratio of $D$ to $S$ amplitudes considered in this work. v's. the mixing angle $\theta_{k} \ldots$. . . .

5.1 Graph of the $\gamma \rightarrow \pi^{+} \pi^{-}$total cross-section vs. $\sqrt{5}$. for the tree-level scalar QED and BDI predictions. and recent data . . . . . . . il

5.2 The tre-level Feyman diagrams for the scalar QED interaction $99 \rightarrow$ $\pi^{+} \pi^{-} \ldots \ldots \ldots \ldots \ldots \ldots$

5.3 The geometry of the $7 \rightarrow \rightarrow \pi^{+} \pi^{-}$interaction in the CM frame. . . . T4

5.4 Ciraph of the $99 \rightarrow \pi^{+} \pi^{-}$tree-level scalar QED cross-sections is. $\sqrt{s} \quad 76$

5.5 Example diagrams of the two classes of interactions included in the quark model used to find the effective potentials . . . . . . . . . it

i.t Graphs of the $\pi-\pi$ potentials used in this work vs. $r \ldots \ldots$. . . . . .

5.T Ciraphs of the $I=2 \pi \pi$ scattering phase shift vs. $\sqrt{s}$. for our predictions, the quark model prediction. and experimental data . . . . . .

is (iraphs of the $I=0 \pi \pi$ scattering phase shift $v$ s. $\sqrt{s}$. for our predictions. the quark model prediction. and experimental data . . . . . . 
5.9 Graph of the 7$) \rightarrow \pi^{+} \pi^{-}$total cross-section is. $\sqrt{5}$ for our predictions. the tree-level scalar QED prediction. and experimental data . . . . . s!

5.10 Graphs of the $97 \rightarrow \pi^{0} \pi^{0}$ cross-section is. 5 . for our predictions (cases $A . A^{\prime} . B 1$ and $\left.B 1^{\prime}\right)$ and experimental data ............ (n)

5.11 Graphs of the $7 \rightarrow \rightarrow \pi^{0} \pi^{0}$ cross-section vs. $\sqrt{s}$. for our pretictions (cases $B$ and $B^{\prime}$ ) and experimental data . . . . . . . . . . . . 


\section{Chapter 1}

\section{Introduction}

Particle physirs is the study of the particles that make up the universe, and the interactions that take place between them. It is also referred to as elementary particle physics. by which we mean that we would ideally like to understand the universe it terms of its "elenientary" constituents (should such things exist). that are not romposed of other particles.

Why study particle physics? The questions "What is the universe made of?" and "How does it work"* are of fundamental interest for their own sake. In addition. understanding the universe as it is now might help us answer the question "Where did it come from?"

Aside from these weighty questions, what about immediate material benefits? Like much of fundamental research. it is impossible to know now s. hat benefits might lwe realized from particle physics. However. it is worth noting that in 1897 the quest to underst and the universe led to the discovery of the electron by J.J. Thompson. It is safe to say that this early particle physicist could not have predicted the huge effert his work would have on humanity. 


\subsection{The Standard Model of Particle Physics}

We assume that the reader is familiar with modern physics. Familiarity with the lield of particle physics would also be useful. hut hopefully not mecessary: in addition lo the introduction to the field that follows. Aplendix A contains descriptions of anme of the terms and tools of particle physics that may he useful to sperialists in other fields.

\subsubsection{The Particles that Constitute Matter}

At present, the elementary particles that make up matter are thought te. lee the" quarks (six of them, plus their antiparticles) and the leptoms (again. six of therti. plus antiparticles). We think of them as elementary becaluse. so far. We des not have any indication that they are not. The quarks are the up $(u)$. Charm (d) alld (1) (f). all with electric charge $+\frac{2}{3}$ (on a scale where the electron has chargo - 1). and the diown $(d)$. strange (s) and bottom (b). all with charge $-\frac{1}{3}$. The leptoms are the elent ront (a). mu $(\mu)$ and tan $(\tau)$. all with charge -1 , and the three correnpomeling mentrimes, ts.

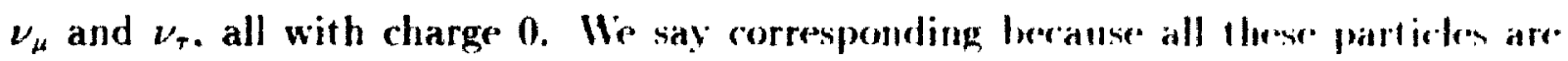
usually thought of as being arranged in doublets.

$$
\begin{aligned}
& \text { Quarks: } \begin{array}{l}
\operatorname{charg} c+\frac{2}{3}: \\
\operatorname{charg} v-\frac{1}{3}:
\end{array}\left(\begin{array}{l}
a \\
d
\end{array}\right) \cdot\left(\begin{array}{l}
r \\
v
\end{array}\right) \cdot\left(\begin{array}{l}
1 \\
b
\end{array}\right) \text {. }
\end{aligned}
$$

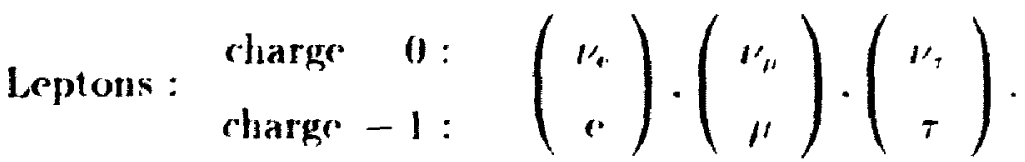

As one moves to the right in the first. second and fourth wow'. the partic lem luenone

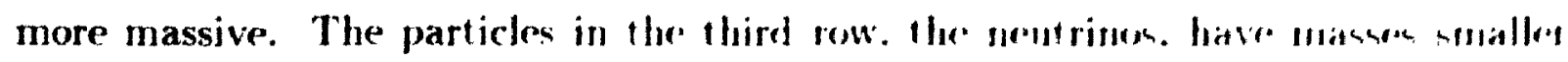

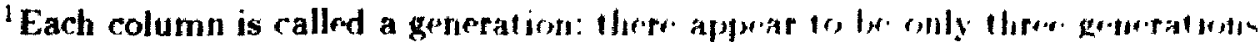




\begin{tabular}{|c|c|c|c|c|}
\hline \multirow[t]{2}{*}{ Quitrk } & \multicolumn{2}{|c|}{ Quark Mass } & \multirow[t]{2}{*}{ Lepton } & \multirow[t]{2}{*}{ Lepton Mass } \\
\hline & Current & Const ituent & & \\
\hline$u$ & $210 \times 116 . \mathrm{V}^{7}$ & $\sim 300 \mathrm{Mel}$ & $1 \%$ & $<5.1 \cdot .1(9.5 \%(1)$ \\
\hline$r$ & 1.0 to $1.6 \mathrm{Gel}$ & $\sim 1.5 \mathrm{Col}$ & $I_{\mu}$ & $<0.16 \mathrm{M} \cdot \mathrm{V}(90 \%$ ('L) \\
\hline 1 & $180 \pm 12$ (iclo & $\sim 1 \times 06$ ir. & $y_{T}$ & $<31 \mathrm{MCl}^{\circ}(95 \%$ ('L) \\
\hline$d$ & $51015 \mathrm{Mer}$ & $\sim 300 \mathrm{MeV}$ & c & $0.51099906(15) \mathrm{M}\left(\mathrm{I}^{\circ}\right.$ \\
\hline$\rightarrow$ & 100 to 300 Mel & $\sim 500 \mathrm{Mel}$ & $\mu$ & 105.658389(34) Mel \\
\hline$b$ & $4.1104 .5 \mathrm{Gel}$ & $\sim 5$ (ifl & $\tau$ & $1775.1_{-0.5}^{+0.4} \mathrm{Mel}$ \\
\hline
\end{tabular}

Table 1.1: Masses of the quarks and leptons. The numbers in parentheses denote the 1 standard deviation uncertainty in the end digits of the value. The \pm errors are also I standard deviation uncertainties. (L means confidence limit (e.g. Wre are 95\% sure that $m_{1 \cdot}<5.1 \times(1)$.

than the experiments can currently measure. and may be massless. The present experimental values of the masses are listed in Table 1.1 [1]. Two different masses are listed for the quarks. The rurrent mass is the mass that appears in the Lagrangian describing the strong interaction (see below). The constituent mass is the effective mass the quark has when it is bound inside a hadron the hadrons are the st rongly interacting particles see below) the numbers given are approximate because they depend on the hadron model used.

Assoriated with each type of quark and lepton is its antiparticle. which has the same mass. but opposite quantum numbers (such as charget. Antiparticles are denoted by an overbar (e.g. u). Each type of quark or lepton is referred to as a

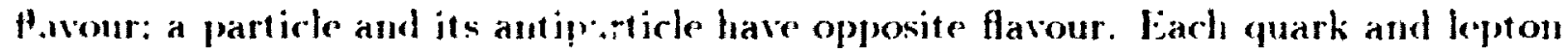
has an int rinsic angular momentum. called spin. of $\frac{1}{2}$. Inaking them fermions (they olery Fermi-Dirac statistics, which hold for partickes of half-integral spin).

In addition to their electric charge, each quark has an additional "charge" referred

\footnotetext{
"Note that whe masses in Table 1.1 are given in units of energ! (el). When the unit of mans would normally be $\cdot C / r^{2}$. We are following thr particle physics convention of settung $c \equiv h \equiv 1$. whirh expresses mass and monent um in units of energy. and length and time in unit of energy ${ }^{-1}$. When comverting from these 'natural units' to more standard units at the end of a calculation, fartors of $h$ and a are inserted as neered to give the required units.
} 
to as colour (but having absolutely nothing to do with the columes of the everydity. world). There are three possible values of a colour charget, plus the three anti-colours of the antiquarks. It appears to be a property of nature that coloured alijects cammet exist freely by themselves. so quarks are confined inside hadrons in configurations that produce an objert with no net colour.

\subsubsection{The Interactions}

There are four different types of interactions that take place beet ween. the farticter: to each of them there corresponds one or more gange howons the' whe! Buse Winstein statisties. which hold for particles of integral spinl. a type of particle that carries the effects of the interaction when it is exchanged between the twe particke involved.

The elect romagnetic interaction ocrurs between objects with electric rlatge. in cluding neut ral particles composed of charged const it uchts (e.g. He Ment ron inlerate via its magnetic moment). It is carried by the photons? which is massless amd has a spin of 1 (making it a vector particle). As a quantum of elect remagnetic ravlia tion. it will be most familiar to the reader as visible light. The theory ponerning the electromagnetic interaction is called Quantum Flectrodynamion (Q $(2))$.

The weak interaction occurs betwern leptoms and quarks, and involites llie in teracting particles being transformed into theje partmers in the dombluts af partirles

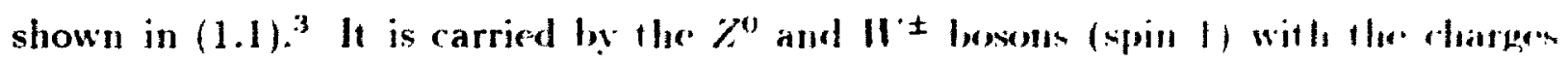

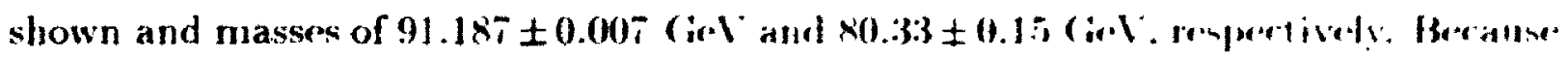
the weak interaction deals with the flavour of the involved partieles. flue therery that

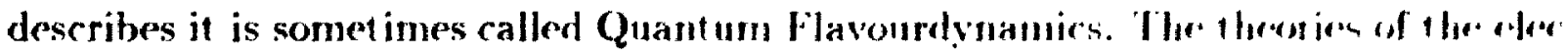

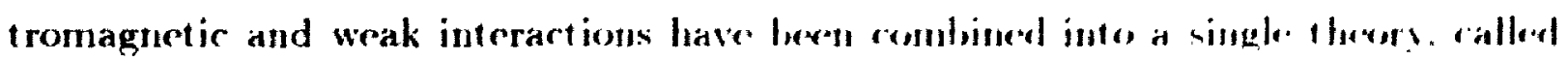

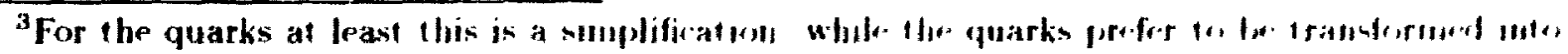

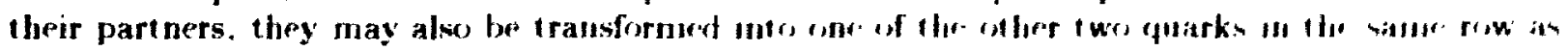

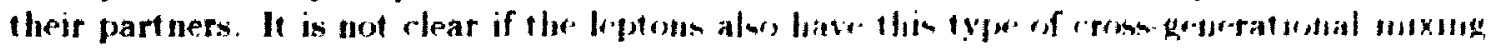


the Elertroweak Theory: The weak interaction is the cause of certain decays. such as the decay of the nentron $\mathrm{n} \rightarrow$ per te. $^{-}$

The st rong interaction occurs between coloured objects. including colourless particles composed of coloured constituents. It is carried by eight gluons $g$. which have spin 1. no mass and no charge. However, they themselves have colour, which complicutes the theory of the strong interaction. Quantum ('hromodynamics (QCD). The strong interaction binds the quarks together to form hadrons. including the nucleons. The residual strong forces between the colourless nucleons bind the nucleus toget her.

The particles and interactions discussed above. and a spontaneous simmetry breaking mechanism (called the Higgs mechanism) that gives the fermions their masses make up what is called the Standard Model of Particle Physies. The Higgs urechanism gives rise to the Higgs boson. a massive ( $>58.4$ Gel. $95 \%$ (1.). neut ral scalar (spin 0) particle. which has not yet been seen.

The remaining interaction. gravity: is well described by a classical theory. Cieneral Relativity, but so far no way has been found to incorporate it into a quantum field theory (such as the elect roweak theory or Q( D). That has not prevented physicists from naming its hypothetical carrier the graviton it would be massless, neut ral. and have' spin 2.

One drawback of the standard model is that it has $19(23)$ if the neut rinos have mass) parameters that must be fitted to the data (e.g. the 12 quark and lepton masses). This doesn't mean that the theory is wrong. but suggests that there is probably a more complete theory ont there. The electroweak theory has been extremely successful; despite extensive experimental programs designed to test it, no stat istically significant deviations from it have been found.

(alculations in the electroweak theory are carried out pert urbatively. in powers of the finc structure constant $a=1 /(135.0359895 \pm 0.0000061)$ for a nomentum transfer of zero (at higher monent um transfers $a$ increases somewhat: at the $Z$ mass 
it is approximately 1/12s). Becanse $n$ is small. the perturbation thery converges reasonably well. However. for $Q(')$. the situation is rather different. Beratise the gluons carry a colour chatge. they can interact among themselves. leiding lo a cont. pling constant $\alpha_{s}$ that decreasts for larger monentum I ransfer. and increase's i $s$ the nomentum transfer goes towards zero. This behaviour at large monientum irausfer is known as asymptotic freedom. and means that perturbative $Q(1)$ is valul for high momentum transfers, but breaks down at the lower momentum transfers where the coupling becomes strong.

Infort unately. the strong interactions found in thre everyday world the hiuding of quarks into nucleons. and of these into atomic nuclei) ocrur at low erergies (and hence low momentum transfers). At present. lattice methods are the only rigurous way 10 do non-pert urbative calculat ions with $Q(1)$. However. while great prengreso has been made using these techniques. it is not vet clear how lung it will lae unt il retliable results can be obtained. especially for complicated problemes such as the ralculatiun of decay widths of excited states.

\subsection{The Hadrons}

The six quarks and six leptons (phus their attiparticles) may make "up mattr. lut only three of them nake up the exeryday mater aroumd wh. All of the mattel in ouls everyday world is tuade up of atoms. An atom rensists of a degse core. the mirtens, surrounded by a clond of electrons. The structure of the elect rent elend in respemsible for the chemical properties of the atom.

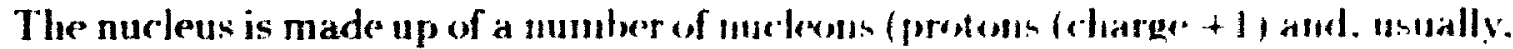

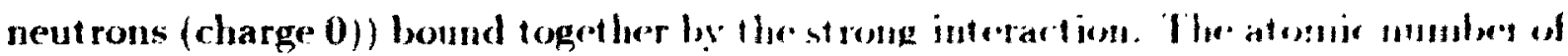

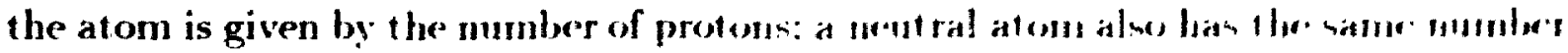
of electrons. The number of nesutrons in rary approximately expai , the mumber of 
protors. Hoth protons and nentrons are mado up of three light quarks: the proton

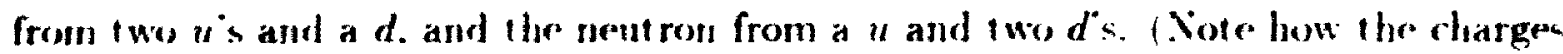
of thre quarks add to give the correct nucleon charges, I Everyday watter is made up of these three constiturnts $\left(0^{-} . " 1\right.$ and $d$ ) because being the light est members of their rows in (1.1). they are stable against decay ${ }^{-4}$ nentrinos are also common. but because there only interact via the weak interartion they interact with everyday matter very rarely. and we are not aware of them.

Protons and neutrons are examples of barcons. one of the two known types of structures which quarks can form. Baryon" ate nade up of thres quarks (qqg): an ant i-haryon would be nade up of three antiquarks. The ot her known $t$ ype of st rurt ure is the meson. which is made up of a quark and an antiquark. gq (so an anti-meson is just a meson). The complicated structure of $Q(D)$ means that groups of quarks can lw bound together only for certain configurations which can have no net colour (so they are in a colour singlet state). It also means that the attractive force between coloured objects is huge. so they are always confined together into colourless objects. This property is called confinement.

In a baryon each quark is a different colour. and the three colours mixed together produce a colourless object. In a meson the quark has a colour and the ant iquark has the corresponding anti-colour to cancel it. again producing a colourless oliject. It may also be possible to have bound qqqqqq or gqqg states. ete. but no observed slates haw been firmly identified as such. Even nore interesting would he glueballs (states consisting only of gluons) or hylbrids (states consisting of both quatks and gluons ser below). Once again. no observed states have been firmly identified with these st ructures. These strongly-interacting states built up of quarks and gluoms are collentively called hadrons.

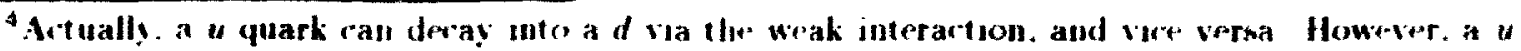
or d always remains. ro taken together they are stahle.
} 
In order to explain gluelatls and hybrids, it helps to introntuce the thux tube picture of Q("D). At highes energies. where perturbative Q(1) is valid. the pin ture of a gluon as a discrete particle exchanged hy quarks is appropriate. Huweere. al lower energies where the coupling becomes st ronger there are indications that a mure appropriate picture is that of tubes of chromenelectric flux commenting the ymaths [2]. These could be thought of as being similar to the lines of an electric field commentieg electrically charged objects. hut becanse of the welf-interacting nat ure of the ghings the lines collapse down to a tube. This pieture incorporates confinement if the flus tubes have a certain energy per unit length. then as the quarkis further alpart lise' energy of the tube would go up linearly. making it impossible .o separal. the quarks.

A meson or baryon is sperified hy the flavours of the quarks. the spane wasefune tion of the quarks. and the wavefunction describiug how the quark spins anmbine. Murh like a hydrogen atom. the space wawefunction ean hate allgular and/or radial

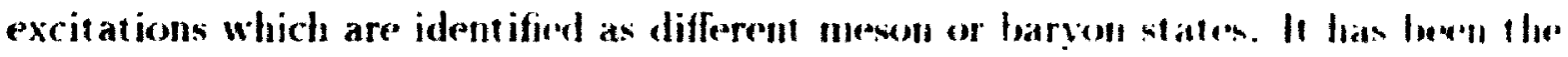
orized (see for example Heference [3]) that the thux-tulue all alde le exriled. either vibrationally (like a vibrating string) or topologionlly (more complicatod lopologiem

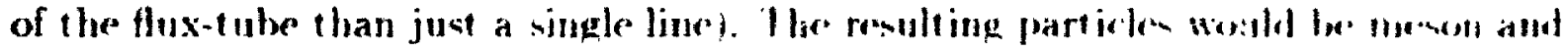
baryon hybrids. The glueball can also he thought of in this picture an a Hux luler connected into a loop. with no quarks present.

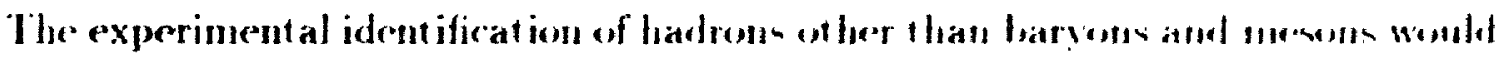
fe. an important confirmation of our understanding of $(\mathrm{Q}$ 'l).

\subsubsection{The Mesons}

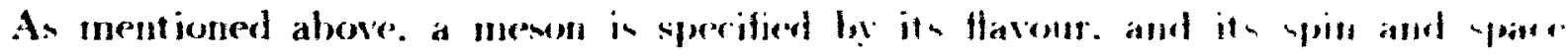
watefunctions. The flacour wavefunetions that we will deal with in thin wenk ale given in Appendix B.2. The spin wavefunetion in particularls simple: 1th I we upion

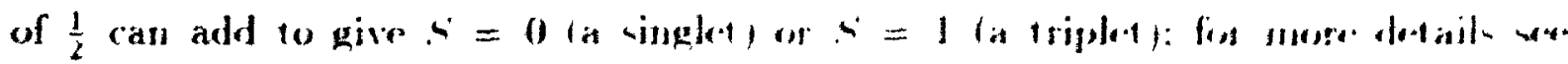


Appenalix 10.3. Like the space wavefunetion of the hydrogen atom. meson spare Wavefunctions also have a radial quant um number $n$ and a quant un mumber for orbital nngular momentum. L. (but unlike the hydrugen atom. "and $L$ are independent ). The total angular momentum is given hy $\vec{J}=\vec{l}+\overrightarrow{5}$. The space and spin state of a meson is often sperified by spert roscopic notation. $n^{2 s+1} L . J$. where the $2 .+1$ is 1 or 3 for the singlet or triplet, respectively. and L in given by a letter (S. P. D. F. (i. H... Wreing 0. 1. 2. 3. 1. 5....). If $n=1$ it will often be neglected in the notation. It is also useful to give the parity and charge conjugation" (where applicable) quant um numbers of th. state these are usually expressed with $J$ as $J$ Fc.

In Table 1.2 we list the known mesom const ructed of the light u. $d$ and s quarks (which are all that we will be comerned with in this thesist according to the l'article Data (iroup [1]. The rows have different space and spin staten and the colums represent differemt flavours. For the $I=1$ and $I=\frac{1}{2}$ column headings the quark states listed are considered to be different charge st ates of the same nueson fand the antiparticles). Hecause of the isospin symmetry between the " and $d$ quarks. For the $t=0$ column the wo mesons in each row are listed together because the proportion of $\frac{1}{2}(u n+d d)$ and su states in each is not loe same for all of the rows. The $c$ assignment in $f^{r}$ only refers to the netural members of the row. The names of the mesoms are related to their flavours, the optional subscript after the natne gives $f$. and the optional number in parenthenes after the name is the mass of the meson in M.'.

\subsubsection{The Quark Model}

Herallice perturbative Q('D) Joes not work at low energies. and non-perturbative calculation have yet to produce detailed results. ne try to calculate the properties of

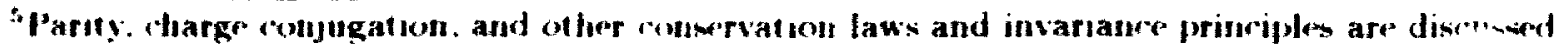
bristly in Appendix A.t
} 


\begin{tabular}{|c|c|c|c|c|}
\hline$n^{25+2} L_{J}$ & $J^{50}$ & $\begin{array}{c}I=1 \\
\left(-u d . \frac{1}{2}(1)(1-d d) . d(u)\right.\end{array}$ & $\begin{array}{c}1=11 \\
\left.1 \frac{1}{1}(1) w+d d\right) \ldots . .\end{array}$ & 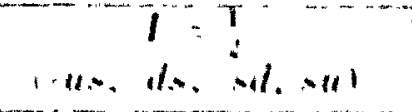 \\
\hline 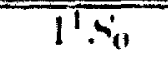 & $0^{-+}$ & $\pi$ & 11.11 & h \\
\hline $1^{3}+4$ & $1^{--}$ & $f^{\prime}$ & -0 & $k \cdot 1+1,1$ \\
\hline$L^{\prime} P_{1}$ & $1^{+-}$ & $b_{1}(1: 2: 3: 5)$ & $h_{1}(1170) . h_{3}(1: 1 \times 0)^{\circ}$ & $h,(1: 70), h_{1}, 1(1,)^{\prime}$ \\
\hline $1^{3} P_{0}$ & $0^{++}$ & $a_{0}(\operatorname{laN}(1)$ & $f_{0}(1): 30(1) . f_{0}(3) \times(0)$ & $h, ; 11: 30)$ \\
\hline $1^{4} P_{1}$ & $1^{++}$ & $a_{1}(1: 260)$ & $f_{1}(1205) . f_{1}(1510)$ & $h_{1} 11: 70, h_{1}(111001+$ \\
\hline $1^{3} P_{2}$ & $2^{++}$ & $a_{2}(1: 3: 2(1)$ & $\left.f_{21} 1270\right) . f_{2}(1525)$ & $H_{2}: 118111$ \\
\hline $\mathrm{I}^{\prime} \mathrm{D}_{2}$ & $2-4$ & $\pi_{2}(1670)$ & 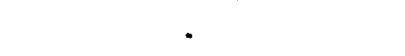 & $k:(17 \%)$ \\
\hline $1^{3} I_{1}$ & $1^{--}$ & $p(1700)$ & . & $h \cdot \|$ tixhl: \\
\hline $1^{3} / I_{2}$ & $2--$ & & • & A.11×:1) \\
\hline $1^{3} \mathrm{Lu}_{4}$ & $3-$ & $p:(169)$ & $-116701.2,1550)$ & $h ; 17 \times 41$ \\
\hline $1^{3} F_{4}$ & $1^{++}$ & $a, 4(20.10)^{5}$ & $f_{1}\left(20.3(0) . f_{1}(20.200)\right.$ & $1 ; 124181$ \\
\hline 245 & $0^{-+}$ & $\pi(1300)$ & 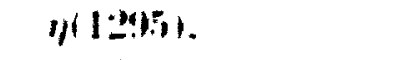 & hllitll: \\
\hline $2 s_{1}$ & $1--$ & $p(11: x)$ & $-111201.016 \times(1)$ & h・(1) $110:$ \\
\hline $2 \cdot I_{2}^{3}$ & $\mid \begin{array}{l}2++ \\
0-+\end{array}$ & & $f_{2}(1 \times 10)^{9} \cdot f_{2}(20)(1)$ & $h:(1 !) \times(1)$ \\
\hline $3^{1} .5$ & $0^{-+}$ & $\pi(1-70)^{2}$ & $1017(i) 1)^{\circ}$ & h $1 \mid \times: B \|^{\circ}$ \\
\hline
\end{tabular}

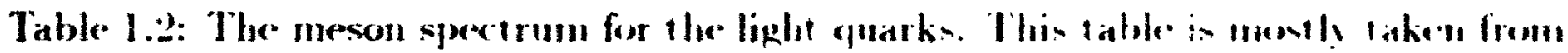
the Keview of Particke Properties [1] by the larticle l)ata (inump.

O These states are not included in the Meson Summaty lahle of the Ken ien of l'at ticle Properties.

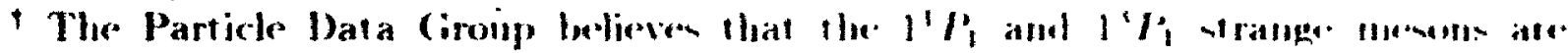

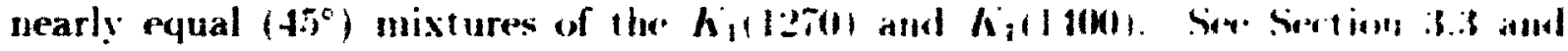
Appendix A.T.

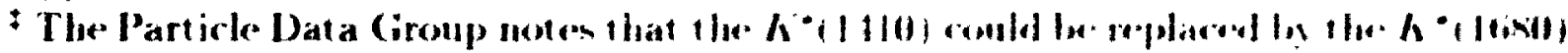
as the 2:"st strange meson.

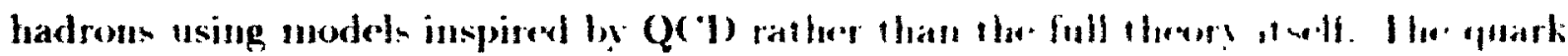

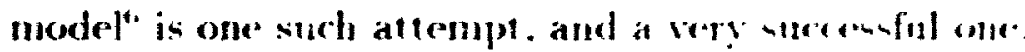

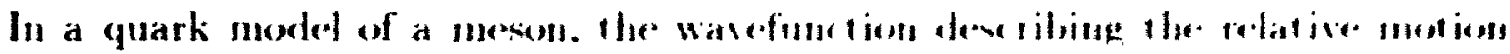
of the quark and antiquark is oblained by whing the Solurinlinger engation nith

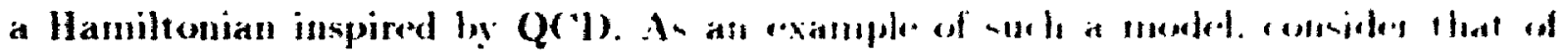

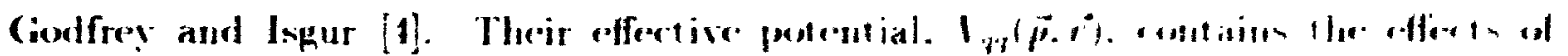

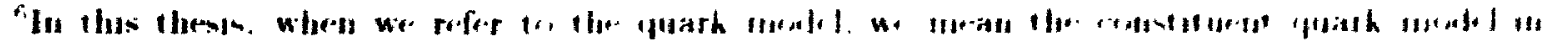
particular.
} 
a loreme vector ome-gluon-exchange interaction at fluort distances and a Lorentz-

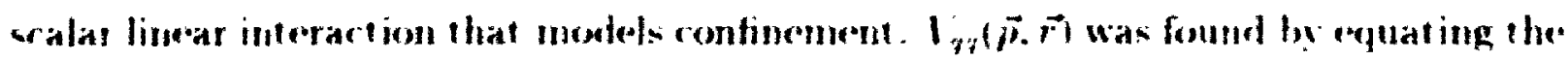
saltering anplitude of fres quarks. waine a wathering kernel with the desired Dirac

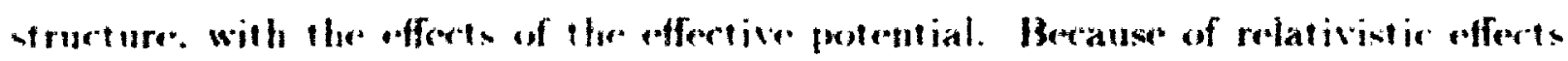
the potential in moment um dependent in addition co heing condinate dependent. To

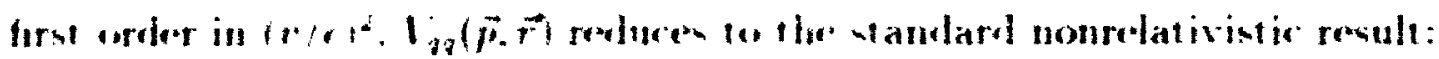

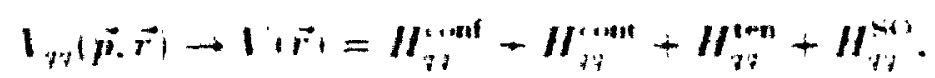

whenge

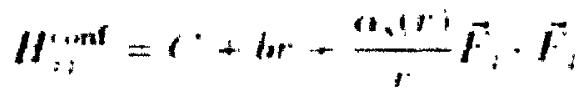

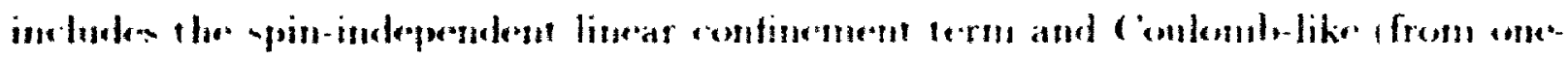
gluen exrliange interaction.

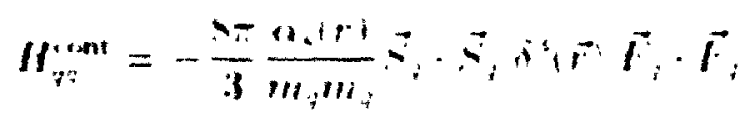

is the contact part of the colour-hypertine interaction.

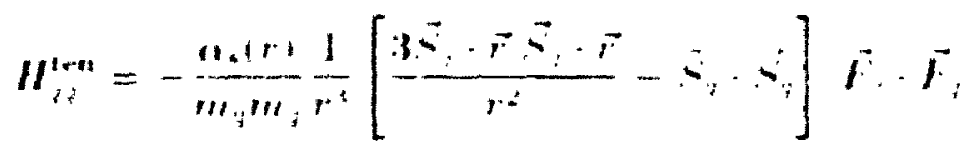

is the tenser part of the colour tiy perfime interaction. and

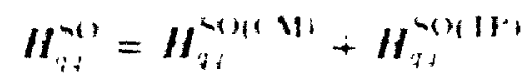

is the spin-arbit interaction with

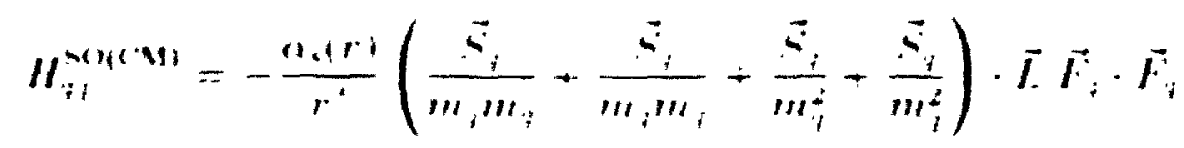




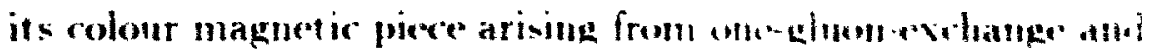

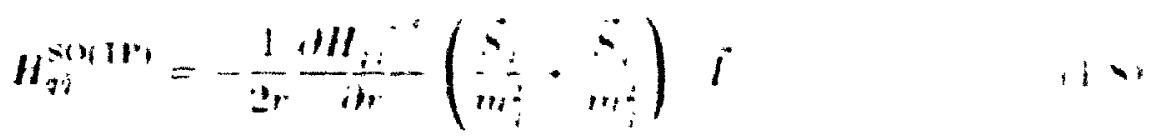

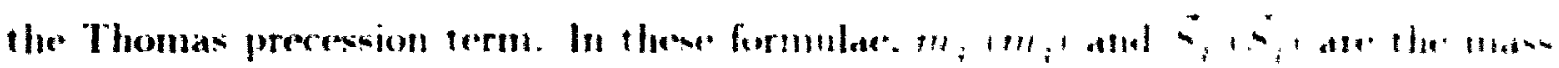

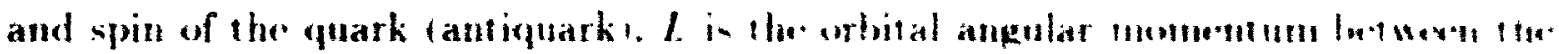

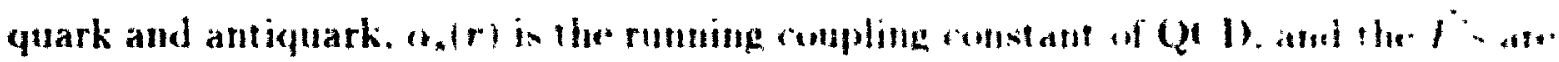

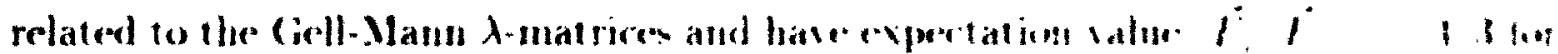
a meson.

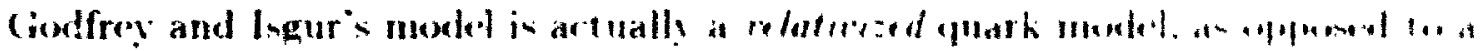

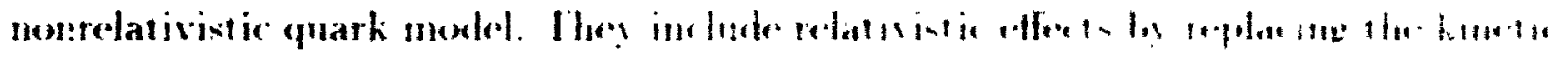

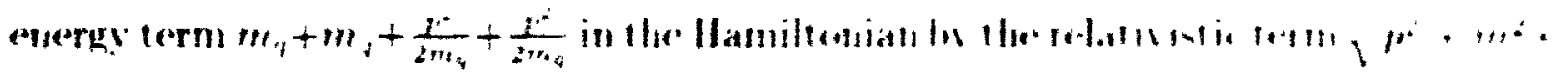

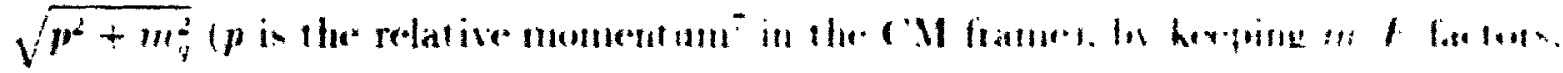

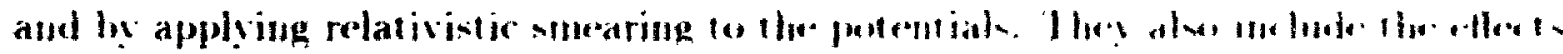
of annihilation interartions via gluons for lle. incoralat menente

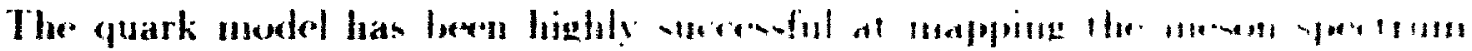

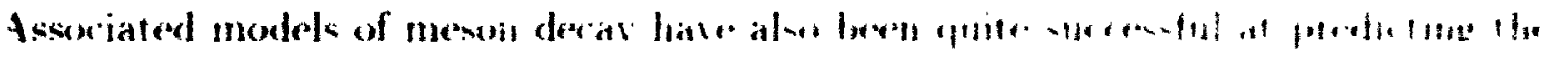
decays i. i.

\subsection{The Work of this Thesis}

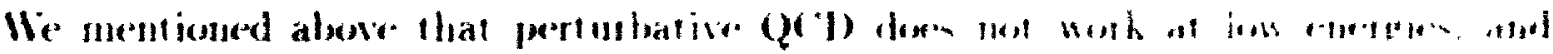

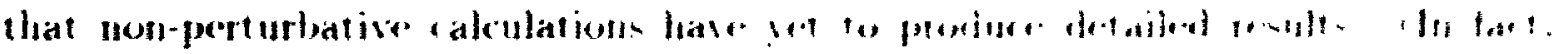

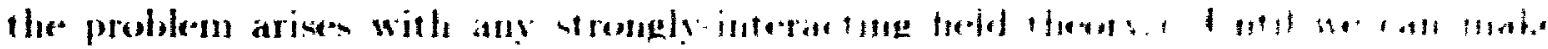

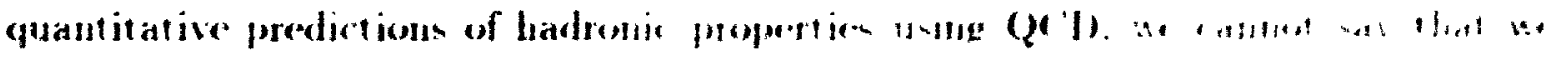

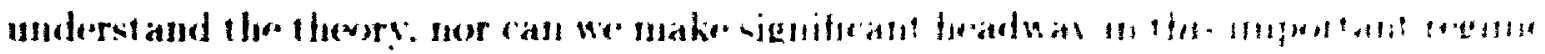

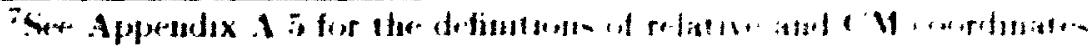


In order to provide some quantitative predictions for the low-energy regime, an industry has sprung up that calculates hadron properties by using models inspired by QCD, rather than the full theory iself. There are three purposes of such a program. The first is to s'ed light on the dynamics of the model, and hence (hopefully) on QCD, by determining which facets of the model are important in predicting resulis that agree with experiment. The second is to understand the properties of the hadrons themselves. The third is to use these calculated properties to map the hadron spectrum. If we understand the meson and baryon spectra well. and there are extra states that do not fit into them, then th:se states must be something new (hybrids, glueballs, etc.)! Confirmation of these extra states would then feed back to the first purpose, understanding the dynamies of QCD.

This thesis deals with properties of mesons calculated in the quark model. which Is one of these attempts to approximate QCD. The meson spectrum. with its large number of states to be studied, provides an excellent testing ground for our understanding of QCD. We examine two general areas in the quark model: models of meson decay. and final state interactions.

In the first area the properties we calculate are the decay widths ${ }^{\mathrm{b}}$ for the strong decays of mesons. Because meson resonances are studied experimentally by examining the particles to which they decay. decay widths are vital in their identification. As an example of this we study the identity of the $f_{4}(2220)$ state - although tentatively identified as the ${ }^{3} F_{1}$ ss meson by the Particle Data Gloup (see Table 1.2). its identity has been uncertain since its dikcovery in 1983 . We also investigate the mixing ${ }^{9}$ between the $h_{1}(1270)$ and $h_{1}(1400)$ mesons. in order to see if the dynamics of the model are responsible for the mixing. or whether it occurs via a separate mechanism that has not yet been included. This is done by comparing the models" predictions

\footnotetext{
"See Appendix A.1 for an introduction to decay widths. resonances and cross-sections.

"See Appendix A.7 for in int roduction to mixing.
} 
for the decay widths of these mesons to experimental data. These works have already been published [6. 7].

In the second area we investigate the effects of the final state interactions in the reaction $\gamma \rightarrow \pi \pi$. This is again an attempt to understand the quark model dynamics responsible for the interaction. In addition. if we understand the situation in $\gamma \gamma \rightarrow$ two pseudoscalar ${ }^{10}$ mesons, we can then approach the situation in $\gamma \rightarrow$ (wo vector mesons with more confidence: the structures seen in the cross-sections of thes: processes are not well understood and are a long-standing puzzle.

In Chapter 2 we describe in detail the models of meson decay we are interesterl in. and the means by which we applied them. In Chapter 3 we fit the free parameter of the models. and investigate a number of choices that must be made in their application. We then apply the models to the first two chosen problems. In ('hapter 1 wo describe in detail the techniques we use for calculating the efferts of final state interartions. In Chapter 5 we apply the techniques to our third chosen problem. In (hapher 6 we conclude.

\footnotetext{
${ }^{10}$ A pseudoscalar particle has $f^{F}=0^{-}$, as opposed to a soralar which has $j^{r}=10^{t}$
} 


\section{Chapter 2}

\section{Two Models of Meson Decay}

\subsection{The ${ }^{3} P_{0}$ Model of Meson Decay}

The ${ }^{3} P_{0}$ model $[8,9]$, also known as the Quark-Pair Creation (QPC) model, is applicable to OZI-allowed' strong decays of a meson into two other mesons, which are expected to be the dominarit decay modes of a meson if they are allowed. It can also be used to describe two-body OZI-allowed strong decays of other hadrons.

In this model, meson decay occurs when a quark-antiquark pair (labelled 3 and 4) is produced from the vacuum in a state suitable for quark rearrangement to occur. as in Figure 2.1. The created pair will have the quantum numbers of the vacuum, $J^{\text {TC }}=0^{++}$. suggesting that they are in a ${ }^{3} P_{0}$ state. There is one undetermined parameter $\gamma$ in the model - it represents the probability that a quark-antiquark pair will be created from the vacuum. The pair is assumed to be created in an SU(N) flavour singlet. where the number of flavours $\mathbf{N}$ is arbitrary, since changing it will just rescale the value of $\gamma$ needed (see Appendix D.2). The rest of the model is just the description of the overlap of the initial meson $(A)$ and the created pair (sometimes

\footnotetext{
${ }^{1}$ See Appendix A.6 for an explanation of the OZI rule.
} 

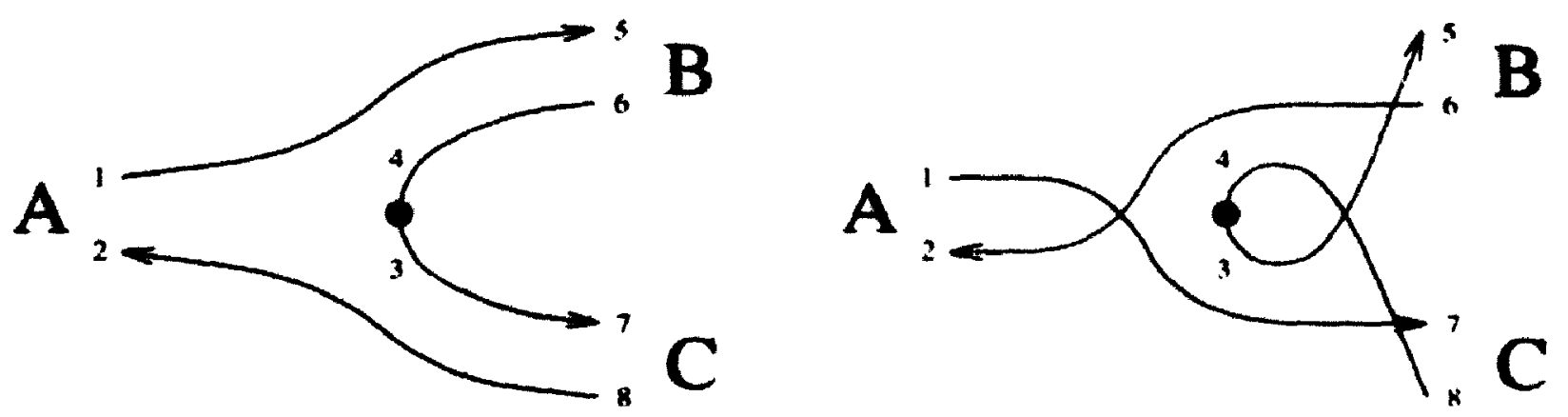

Figure 2.1: The two possible diagrams contributing to the meson decay $A \rightarrow I B 6^{\circ}$ in the ${ }^{3} P_{0}$ model. In many cases only one of these diagrams will contribute.

referred to by 0 ) with the two final mesons ( $\left.B, C^{\circ}\right)$. to calculate the probalility that rearrangement (and hence decay) will occur.

For the meson wavefunction we use a mock meson definal by [10]

$$
\begin{aligned}
& \left|A\left(n_{A}{ }^{2 S_{A}+1} L_{A} J_{A} M_{J_{A}}\right)\left(\vec{P}_{A}\right)\right\rangle \equiv \sqrt{2 E_{A}} \sum_{M_{L_{A}} \cdot M_{E_{A}}}\left(L_{A} M_{L_{A}} \dot{S}_{A} M_{S_{A}}\left|J_{A} M_{J_{A}}\right\rangle\right. \\
& \times \int d^{3} \vec{p}_{A} \psi_{n_{A} L_{A}} M_{L_{A}}\left(\vec{p}_{A}\right) l_{S_{A} M_{-A}}^{12} \theta_{A}^{12} \omega_{A}^{12} \\
& \times\left|q_{1}\left(\frac{m_{1}}{m_{1}+m_{2}} \vec{P}_{A}+\vec{P}_{A}\right) q_{2}\left(\frac{m_{2}}{m_{1}+m_{2}} \vec{P}_{A}-\vec{P}_{A}\right)\right\rangle .
\end{aligned}
$$

Note that given our field-theory conventions of $A$ ppendix ('. the mock meson is nor malized relativistically to

$$
\left.\left\langle A\left(n_{A}{ }^{2 S_{A}+1} L_{A} J_{A} M_{J_{A}}\right)(\vec{P})\right| A\left(n_{A}{ }^{2 S_{A}+1} L_{A} J_{A} M_{I_{A}}\right)(\vec{P})\right)=2 E_{A} \delta^{3}\left(\vec{P}-\overrightarrow{P^{\prime}}\right)
$$

hut uses nonrelativistic spinors and $C M$ coordinates. The moson has ( $M$ momorntum $\vec{P}_{A}$. and the relative momentum of the qu pair $\vec{p}_{A}$ is integrated over all values. The other quantities are as follows: $n_{A}$ is the radial quantum number: $\left|I_{A}, M_{L_{A}}\right\rangle$. $\left|S_{A}, M_{S_{A}}\right\rangle$ and $\left|J_{A}, M_{J_{A}}\right\rangle$ are the quantum numbers of the orbital angular anomen. tum between the two quarks. the total spin of the two quarks. and the total angular

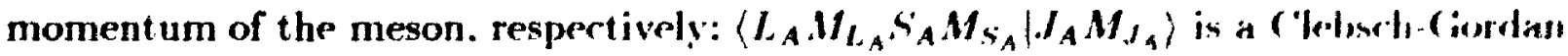


coefficient ${ }^{2} ; E_{A}$ is the total energy of the meson; $\chi_{S_{A}}^{12} M_{S_{A}}, \phi_{A}^{12}$ and $\omega_{A}^{12}$ are the appropriate factors for combining the quark spins to obtain $\left|S_{A}, M_{S_{A}}\right\rangle$. the flavours to obtain the correct meson flavour, and the colours to obtain a colour singlet. respectively:

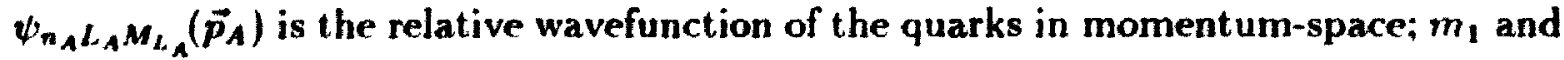
$m_{2}$ are the masses of the quark and antiquark respectively; and finally, $\left|q_{1}\left(\overrightarrow{p_{1}}\right) \vec{q}_{2}\left(\overrightarrow{p_{2}}\right)\right\rangle$ is the basic state of a free quark and antiquark.

Now we can consider the decay $A \rightarrow B C$. Define the $\mathrm{S}$ matrix

$$
S \equiv I-2 \pi i \delta\left(E_{f}-E_{i}\right) T
$$

and then

$$
\langle f|T| i\rangle \equiv \delta^{3}\left(\vec{P}_{f}-\vec{P}_{1}\right) M^{M J_{A} M_{J_{B}} M_{J_{C}}}
$$

whicl gives, using relativistic phase space ${ }^{3}$ and a meson wavefunction normalized as in Ey. 2.2. the decay width in the CM frame

$$
\Gamma=\pi^{2} \frac{P}{M_{A}^{2}} \frac{S}{\left(2 J_{A}+1\right)} \sum_{M_{J_{A}}, M J_{J_{B}}, M_{J_{C}}} \mid M^{\left.M J_{A} M_{J_{B}} M_{J_{C}}\right|^{2}}
$$

Here $P$ is the magnitude of the moment um of either outgoing meson. $M Y_{A}$ is the mass of meson $A . S \equiv 1 /\left(1+\delta_{B C}\right)$ is a statistical factor that is needed if $B$ and $C^{*}$ are identical particles, and $M^{M J_{A} M_{J_{B}} M_{J_{C}}}$ is the decay amplitude.

For the transition operator we use

$T=-3 \gamma \sum_{m}\langle 1 m 1-m \mid 00\rangle \int \mathrm{d}^{3} \vec{p}_{3} \mathrm{~d}^{3} \vec{p}_{4} \delta^{3}\left(\vec{p}_{3}+\vec{p}_{4}\right) \mathcal{Y}_{1}^{m}\left(\frac{\beta_{3}-\beta_{4}}{2}\right) \backslash \backslash_{1-m}^{34} \phi_{0}^{34} \omega_{0}^{34} b_{3}^{\dagger}\left(p_{3}\right) d_{4}^{\dagger}\left(p_{4}\right)$.

Here the momenta of both the created quark (3) and antiquark (4) are integrated over

\footnotetext{
"See Appendix A.3 for an explanation of (lebsrtz-Gordan coefficients and Wigner $n j$ symbols.

${ }^{3}$ Note that we have used relativistic phase space (leading to Eq. 2.5) and a relativistic normalization (Eq. 2.2). We will also consider other choices of phase space/normalization in Section 3.1.
} 
all values, with the constraint that their total moment um is zero. Other quantities are as follows: $\gamma$ is the one undetermined parameter in the modelt: $b_{3}^{\dagger}\left(p_{3}\right)$ and $d_{4}^{\dagger}\left(p_{4}\right)$ are the creation operators of the created quark and antiquark, respectively: $\mathcal{J}_{i}^{m}(\vec{p}) \equiv$ $p^{l} Y_{l}^{m}\left(\theta_{p}, \phi_{p}\right)$ is a solid harmonic that gives the momentum-space distribution of the created pair ${ }^{5}$ and the other quantities are as in Eq. 2.1. Here the spins and relative orbital angular momentum of the created quark and antiquark are combined in at " $P_{6}$ state to give the pair the overall $J^{\text {rC }}=0^{++}$quantum numbers.

Combining Eqs. 2.1, 2.4 and 2.6 gives for the amplitude in the ( $M$ frame (affer doing the colour wavefunction overlap. and transforming one of the spin wavefunction overlaps - see Appendix D):

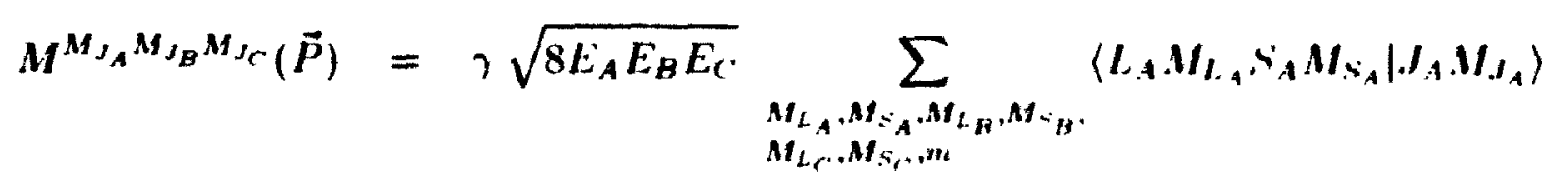

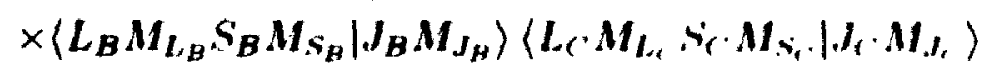

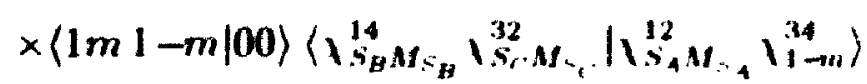

$$
\begin{aligned}
& \times\left[\left\langle\phi_{B}^{14} \phi_{C}^{32} \mid \phi_{A}^{12} \phi_{0}^{34}\right\rangle /\left(\vec{P}, m_{1}, m_{2}, m_{3}\right)\right. \\
& \left.+(-1)^{1+s_{A}+s_{B}+s_{1} \cdot}\left\langle\phi_{B}^{32} \phi_{C}^{14} \cdot \mid \phi_{A}^{12} \phi_{1}^{34}\right\rangle I\left(-\overrightarrow{\boldsymbol{r}} \cdot m_{2}, m_{1}, m_{3}\right)\right] .
\end{aligned}
$$

The two terms in the last factor correspond to the two possible diagrams in ligure 2.1 - in the first diagram the quark in $A$ ends up $B$; in the second it ends up in (. The superscripts within the spin and flavour wavefunction overlaps serve to identify the (anti)quarks on the left side with the corresponding (anti)quarks on the right side.

\footnotetext{
${ }^{4}$ Our value of $\gamma$ is higher than that used by Kokoski and Isgur [5] by a fartor of $\sqrt{96 \pi}$ dur. to, different field theory conventions, constant factors in $T$, et "The calculated values of the wirltin are, of course, unafiected.

${ }^{5}$ The presence of the solid harmonic can be demonstrated by cxamining the usat rix ateme'ut for

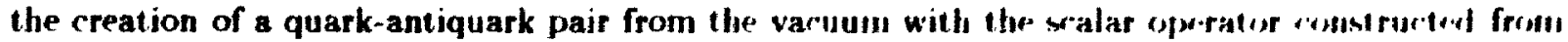

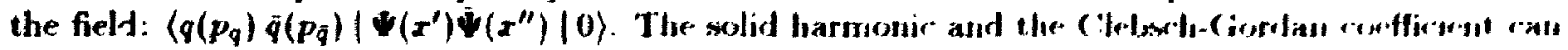
both be extracted from the spinor portion of the result
} 
The momentum space integral $I\left(\vec{P}, m_{1}, m_{2}, m_{3}\right)$ is given by

$$
\begin{aligned}
J\left(\vec{p}, m_{1}, m_{2}, m_{3}\right)= & \int \mathrm{d}^{3} \vec{p} \psi_{n_{B} L_{B} M_{L B}}\left(\frac{m_{3}}{m_{1}+m_{i}^{*}} \vec{p}+\vec{p}\right) \psi_{n_{C} L_{C} M_{L_{C}}^{*}}\left(\frac{m_{3}}{m_{2}+m_{A}} \vec{p}+\vec{p}\right) \\
& \times v_{n_{A} L_{A} M_{L_{A}}}(\vec{P}+\vec{p}) Y_{1}^{m}(\vec{p})
\end{aligned}
$$

where we have taken $\vec{P} \equiv \vec{P}_{H}=-\vec{P}_{C}$.

\subsection{Calculating Meson Decay Widths with the ${ }^{3} P_{0}$ Model}

In order to calculate the decay amplitudes of the ${ }^{3} P_{0}$ model. we use the techniques of Hoberts and Silvestre-Brac [9]. These techniques require that the radial portions of the meson space wavefunctions be expressible in certain functional forms. which encompass simple harmonic oscillator (SHO) wavefunctions. In what follow's. we assume that the radial portions of the wavefunctions are expressed as linear combinations of the first $N+1$ SHO radial wavefunctions (see Appendix B.1).

$$
R_{L_{A}}\left(p_{A}\right)=\sum_{a=0}^{X} d_{a}^{A} R_{n_{A}=a, L_{A}}^{\mathrm{SHO}}\left(p_{A}\right)
$$

The $3_{4}$. elc. found below are the oscillator parameters of the wavefunctions.

The decay amplitudes of Eq. 2.7 are converted to partial wave amplitudes by means of a recoupling calculation (see Appendix E). Then the whole expression for the amplitudes, including the integrals of Eqs. 2.8 and E.4. is converted into a sum over angular moment um quant um numbers. The graphical methods for manipulating angular momentum expressions found in Zare [11] were particularly useful in this 
regard. Our result, very similar to that of Roberts and Silvestre-Mrac, is

$$
\begin{aligned}
& \left.M^{S L}(P)=\right\urcorner \sqrt{\frac{8 E_{A} E_{B} E_{C}}{3}}(-i)^{L_{A}+L_{B}+L_{r}}(-1)^{1+s_{B}+s+J_{B+}+J_{t}}
\end{aligned}
$$

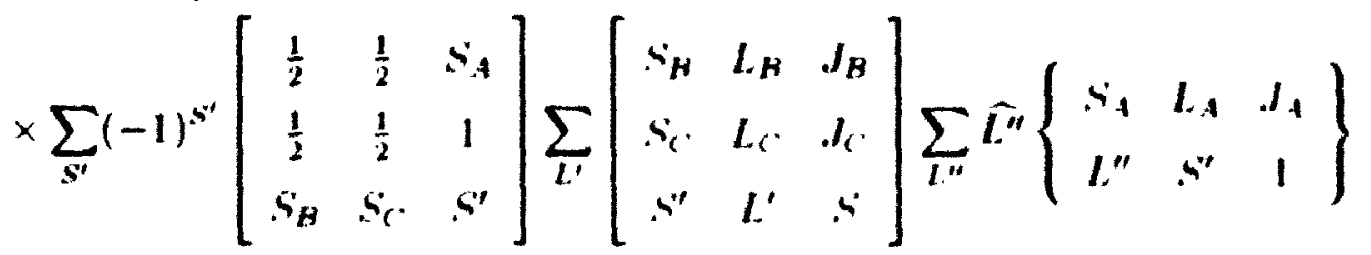

$$
\begin{aligned}
& \times\left\{\begin{array}{lll}
S^{\prime} & L^{\prime} & S \\
L & J_{A} & L^{\prime \prime}
\end{array}\right\}\left[\left\langle\phi_{B}^{14} \phi_{C}^{32} \mid \phi_{A}^{12} \phi_{0}^{34}\right\rangle \in\left(L_{A} \cdot L_{H} \cdot L_{C}, L . L^{\prime} . L^{\prime \prime} . I^{\prime} \cdot m_{1}, m_{2}, \mid m_{A}\right)+\right.
\end{aligned}
$$

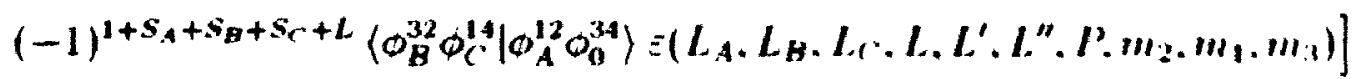

where the modified $9 j$ symbol is defined in terms of the $! !$ symlnol

$$
\left[\begin{array}{ccc}
j_{1} & j_{2} & J_{12} \\
j_{3} & j_{4} & J_{34} \\
J_{13} & J_{24} & J
\end{array}\right] \equiv \widehat{J_{12}} \widehat{J_{34}} \widehat{J_{13}} \widehat{J_{24}}, j\left\{\begin{array}{ccc}
j_{1} & j_{2} & J_{12} \\
j_{3} & j_{4} & J_{34} \\
J_{13} & J_{24} & J
\end{array}\right\}
$$

and where $\hat{\jmath} \equiv \sqrt{2 J+1}$.

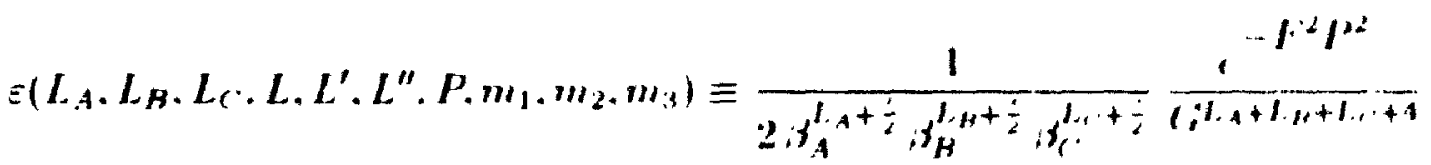

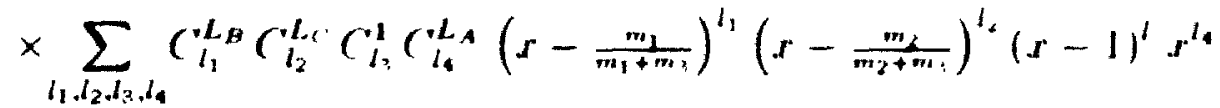

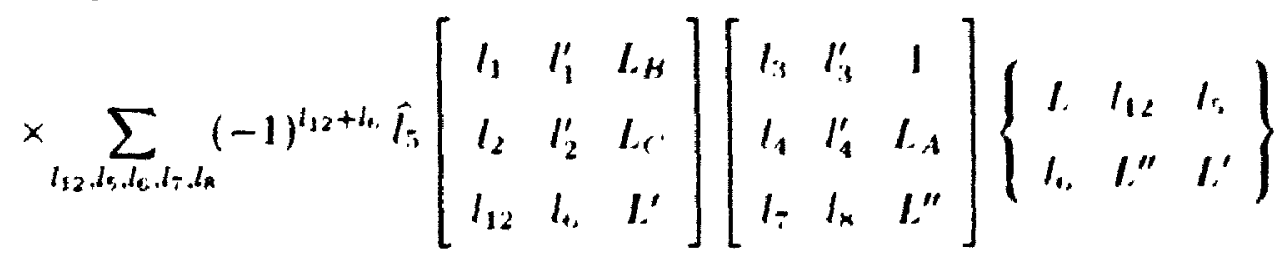

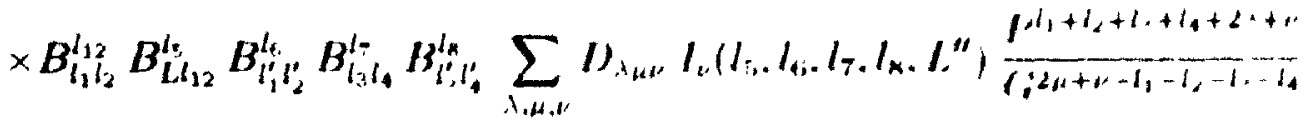


Chapter 2. Two Models of Meson Decay

$$
\begin{aligned}
& \times\left(\frac{l_{1}^{\prime}+l_{2}^{\prime}+l_{3}^{\prime}+l_{4}^{\prime}+2 \mu+\nu+1}{2}\right) ! \\
& F^{\prime 2} \equiv \frac{1}{2}\left[\frac{x^{2}}{\beta_{A}^{2}}+\frac{\left(x-\frac{m_{1}}{m_{i}+m_{3}}\right)^{2}}{\beta_{B}^{2}}+\frac{\left(x-\frac{m_{2}}{m_{2}+m_{\lambda}}\right)^{2}}{\beta_{C}^{2}}\right] \text {. } \\
& r^{2} \equiv \frac{1}{2}\left[\frac{1}{B_{A}^{2}}+\frac{1}{B_{B}^{2}}+\frac{1}{B_{C}^{2}}\right] \\
& r \equiv \frac{\left(\frac{m_{1}}{m_{1}+m_{2}} \beta_{C}^{2}+\frac{m_{2}}{m_{2}+m_{3}} \beta_{B}^{2}\right) B_{A}^{2}}{\beta_{A}^{2} A_{B}^{2}+B_{A}^{2} \beta_{C}^{2}+\beta_{B}^{2} \beta_{C}^{2}} \\
& C_{l_{1}}^{\prime} \equiv \sqrt{\frac{4 \pi(2 l+1) !}{\left(2 l_{1}+1\right) !\left[2\left(l-l_{1}\right)+1\right] !}} \\
& B_{i_{1} l_{2}}^{\prime} \equiv \frac{(-1)^{l}}{\sqrt{4 \pi}} i_{1} i_{2}\left(\begin{array}{lll}
l_{1} & l_{2} & l \\
0 & 0 & 0
\end{array}\right)
\end{aligned}
$$

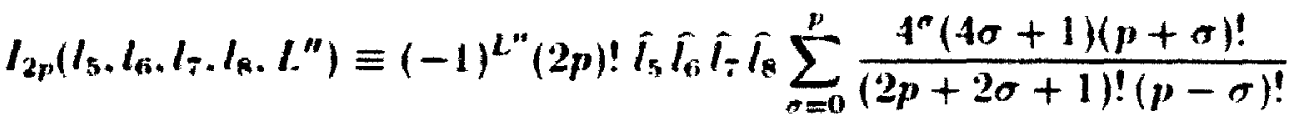

$$
\begin{aligned}
& \times\left(\begin{array}{ccc}
2 \sigma & l_{5} & l_{7} \\
0 & 0 & 0
\end{array}\right)\left(\begin{array}{ccc}
2 \sigma & l_{6} & l_{8} \\
0 & 0 & 0
\end{array}\right)\left\{\begin{array}{lll}
l_{5} & l_{65} & L^{\prime \prime} \\
l_{8} & l_{7} & 2 \sigma
\end{array}\right\} \text {. }
\end{aligned}
$$

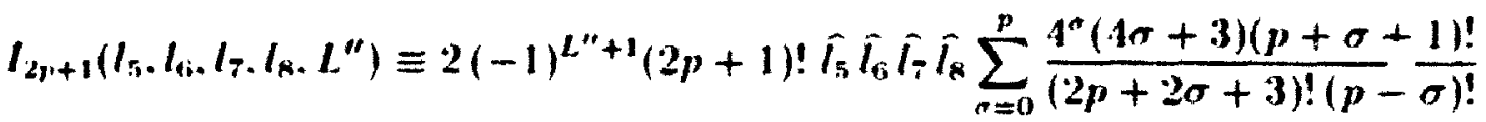

$$
\begin{aligned}
& \times\left(\begin{array}{ccc}
2 \sigma+1 & l_{5} & l_{5} \\
0 & 0 & 0
\end{array}\right)\left(\begin{array}{ccc}
2 \sigma+1 & l_{4 i} & l_{8} \\
0 & 0 & 0
\end{array}\right)\left\{\begin{array}{ccc}
l_{5} & l_{6} & L^{\prime \prime} \\
l_{8} & l_{5} & 2 \sigma+1
\end{array}\right\} .
\end{aligned}
$$

where $I_{1}^{\prime} \equiv L_{B}-I_{1} \cdot l_{2}^{\prime} \equiv L_{C}-I_{2} \cdot l_{3}^{\prime} \equiv 1-I_{3} . I_{4}^{\prime} \equiv L_{A}-I_{4}$ and $D_{\lambda_{\mu}}$ can be ext racted (by equating coetficients of $k^{2 x} q^{2 \mu}(\vec{k} \cdot \vec{q})^{\prime \prime}$ ) from

$$
\begin{aligned}
& \sum_{1, \mu, \alpha} l_{1, \mu+} k^{21} q^{2 \mu}(\vec{k} \cdot \vec{q})^{\nu} \equiv \sum_{a, b, r=0}^{N}(-1)^{a+b+r} d_{a}^{4} d_{b}^{B} d_{r}^{\alpha} \\
& \sqrt{8 a ! b ! c ! \Gamma\left(a+L_{A}+\frac{1}{2}\right) \Gamma\left(b+L_{B}+\frac{3}{2}\right) \Gamma\left(c+L_{C}+\frac{3}{2}\right)} \\
& \times \sum_{m_{a}=0}^{a} \sum_{m_{b}=0}^{h} \sum_{m_{1}=0}^{c}(-1)^{m_{a}+m_{b}+m_{a}} \frac{1}{3_{A}^{2 m_{a}} \cdot 3_{B}^{2 m_{b}} 3_{c}^{2 m_{c}}} \frac{1}{\left(a-m_{b}\right) !\left(b-m_{b}\right) !\left(c-m_{c}\right) !}
\end{aligned}
$$




$$
\begin{aligned}
& \times \frac{1}{\Gamma\left(m_{a}+L_{A}+\frac{3}{2}\right) \Gamma\left(m_{b}+L_{B}+\frac{3}{2}\right) \Gamma\left(m_{1}+L_{C}+\frac{\pi}{2}\right)}
\end{aligned}
$$

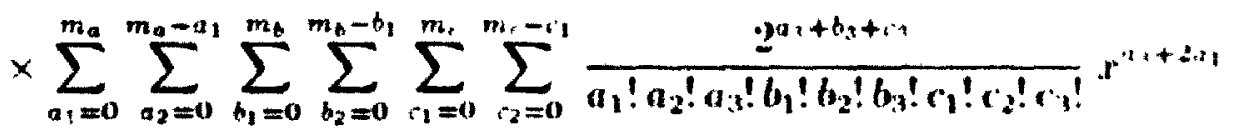

$$
\begin{aligned}
& \times\left(x-\frac{m_{1}}{m_{1}+m_{3}}\right)^{b_{1}+2 b_{1}}\left(x-\frac{m_{2}}{m_{2}+m_{2}}\right)^{a_{1}+2 b_{1}}\left(k^{2}\right)^{a_{1}+b_{1}+c_{1}}\left(q^{2}\right)^{a_{2}+b_{2}+c_{2}}\left(\vec{k} \cdot q^{q_{1}+h_{2}+w_{2}} .\right.
\end{aligned}
$$

where $a_{3} \equiv m_{a}-a_{1}-a_{2} \cdot b_{3} \equiv m_{h}-b_{1}-b_{2}$ and $c_{3} \equiv m_{c}-c_{1}-c_{2}$

These expressions are general: they can bo used for any meson deray where the radial portion of the wavefunctions can be expanded in terms of Sill() radial wavefunctions. In order to obtain symbolic results in terms of the various paraneters, we coded these expressions into routines for the symbolic comput alion parkage Mathe" matica [12]. In principle. these routines are limited only hy the size of the symluslic problem that results, and the available computer resourcess in practice, clenays illvolving mesons with more complicated wavefunctions (i.e. having high I' in lix. 2.4, or high $L$ ) would have to be done numerically. The overlaps of the colour, flavur and spin wavefunctions of the mesons and the created pair are calculated usiug the techniques of Appendix D.

\subsection{The Flux-tube Breaking Model of Meson De- cay}

In the flux-tube picture a meson consists of a quark and antiquark commented ly a tube of chromoelectric flux, which can be treated as a vibrating string. Fin monsm: the string is in its vibrational ground state. (Vibrational excitations of the mtring would correspond to a type of meson hybrid. particles whose existermed have mot yed been confirmed.) Meson decay occurs when the flux-tube breaks at a point. and a 


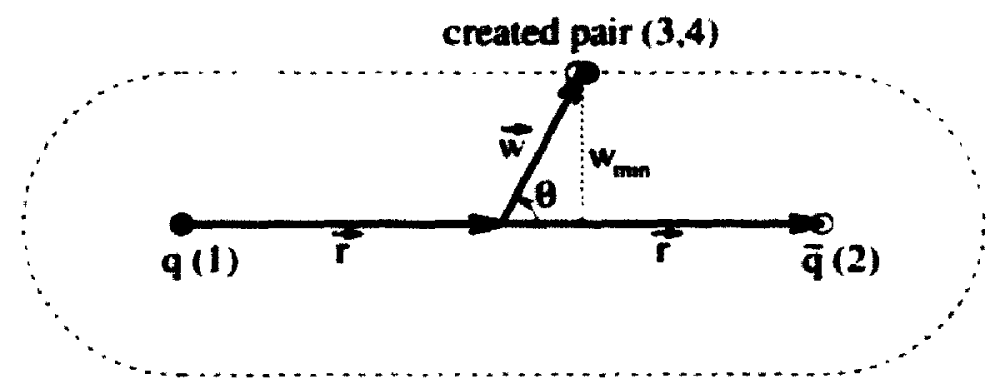

Figure 2.2: The position-space coordinates used in the flux-tube model. The cigarshaped dashed line shows a possible surface of constant $w_{\min }$.

quark-antiquark pair is created from the vacuum to connert to the free ends of the flux-tubes, leaving a final state consisting of two mesons.

The flux-tube breaking model of meson decay is similar to the ${ }^{3} /$ model, but extends it by considering the actual dynamics of the flux-tubes. This is done by including a factor representing the overlap of the flux-tube of the initial meson with those of the two outgoing mesons. Kokoski and lsgur [5] have calculated this factor by treating the flux-tubes as vibrating strings. They approximate the rat her complicated result by replacing the undetermined parameter ? in the ${ }^{3} P_{0}$ model with a function of the location of the created quark-ant iquark pair. and a new undetermined parameter $\gamma_{0}:$

$$
\gamma(\vec{r} \cdot \vec{u})=\gamma_{0} c^{-\frac{1}{2} b u_{\min }^{2}}
$$

Here $b$ is the string tension. where a value of 0.18 (iel' ${ }^{-2}$ is typically used. and $u^{\text {min }}$ is the shortest distance from the line segment connecting the original quark and antiquark to the location at which the new quark-antiquark pair is created from the vacuun (see Figure 2.2):

$$
u_{\min }^{2}=\left\{\begin{array}{ll}
u^{2} \sin ^{2} \theta . & \text { if } r \geq u \cdot|\cos \theta| \\
r^{2}+u^{2}-2 r u \cdot|\cos \theta| . & \text { if } r<u \cdot|\cos \theta|
\end{array} .\right.
$$


To incorporate this into the ${ }^{3} P_{0}$ model. we first Fourier transform Eq. Z.s su that the integral is over position-space. We the'n pull the parameter ? (from fy. 2.5) inside the integral. and replace it by the function of position $\rightarrow(\vec{r}, \vec{u})$. The expression fer the amplitude in the flux-tube model is then the same as that of Eq. 2.T exerpt that ? Is replaced by $\gamma_{0}$. and $I\left(\vec{P}, m_{1}, m_{2}, m_{3}\right)$ is replaced by.

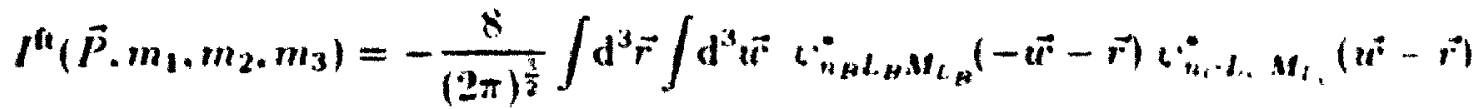

$$
\begin{aligned}
& \times \mathcal{Y}_{1}^{m}\left(\left[\left(\vec{P}+i \vec{r}_{F_{A}}\right) v_{n_{A} L_{A} M_{L A}}\left(\vec{r}_{A}\right)\right]_{\vec{r}_{A}=-2 \vec{r}}\right),-\frac{1}{2} b u_{\min }^{2} i \vec{P} \cdot\left(m_{+} \overrightarrow{\vec{r}}+m \ldots \overrightarrow{r^{\prime}}\right)
\end{aligned}
$$

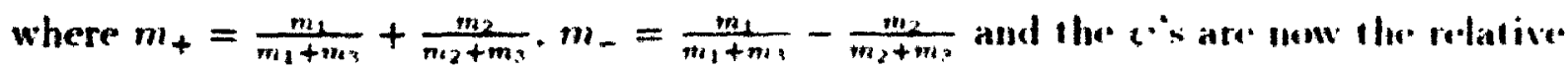
wavefunctions in position-space. The last exponential function in lig. 2.2:1 is the

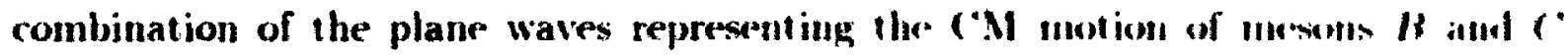
(the plane wave for $A$ is unity. since it is at rest in the ( M framol. It comes from Fourier-transfo ning the delta-functions implicit in He. 2.1 that sperify that the ( MI momenta of the mesons are held tixed to const ant values. Note alwo that we riume to

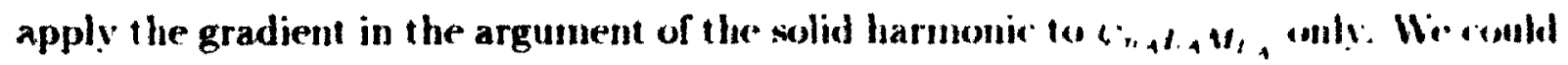

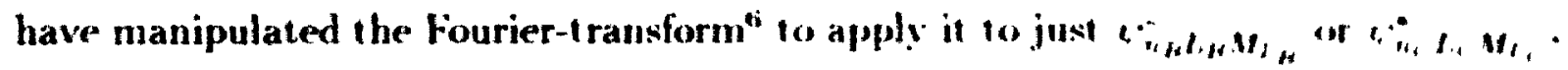
or some combination of all three. In fact since $B$ and $\left(^{\circ}\right.$. Ineing lighter than .1. Irend

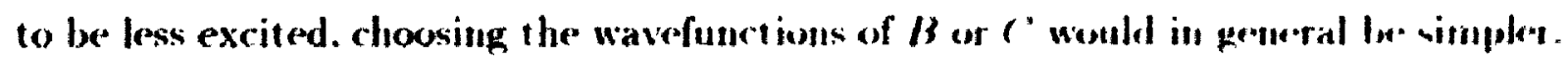

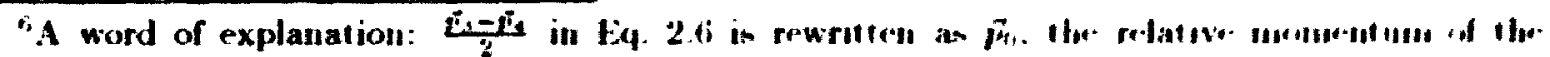

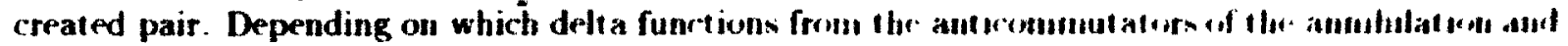

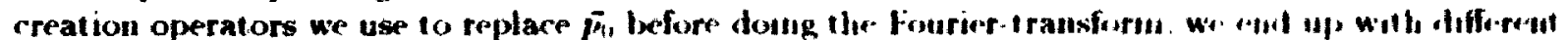

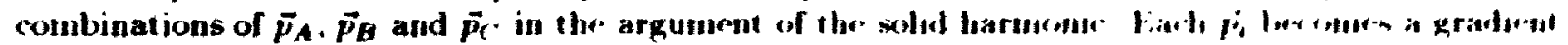
applied to $6, L, M_{2}$, during the Fourner-transform 


\subsection{Calculating Meson Decay Widths with the Flux-tube Breaking Model}

In order to alculate the deray amplitudes of the flux-tube breaking model. there are a number of integrals that must be evaluated: two 3-dimensional integrals from Fa. 2.23 and. if we choose to use the reroupling calculation to convert to partial wave anuplitudes (ser Appendix E), anot ber 2-dimensional integral from Eq. E.A.

We wish to develop general routines to calculate any decay amplitude. This prevents us from making the comnon assumptions that can simplify Eq. 2.23. wuch as unglecting mass differences lxetwern the quarks. rest ricting the orbital angular monenta of each meson fo be 0 or $I$ so that their spherical harmonics an be expressed as dot promets of their argument and a contant vector. or simplifying the definition

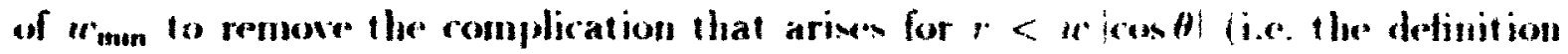
currently used for $r \geq$ u'cost would be used for all values). The main goal of simplifying Ey. 2.2:3 is to allow more of the integrals to be done analy fically, to melure the computer time required for a calculation.

In order to save ourselves two integrations. we cheosed to use the dacolb-llick formula to convert to partial wave amplituden (ser Appendix E). (Rementer that thin involves chosing $\vec{P}$ to lie along $\vdots$ the $z$ axis. $)$ This leaves us with integrals ow

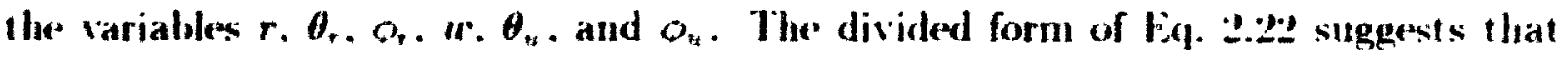
we will not be able to do the integrals over any of the variables on which it depends. This inmediately rules out $r$ and $w$. If $\vec{r}$ and $\vec{a}$ are expressed in the same coordinate system. the angle mel ween them $(\theta)$ is given by

$$
\cos \theta=\sin \theta, \sin \theta_{1} \cos \sin -\omega_{n} 1+\cos \theta_{\cos } \cos \theta
$$

which presents a problem for the other variables as well. The solution is to use the 


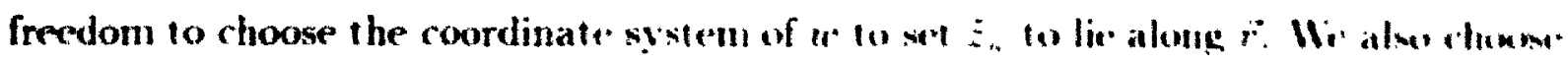

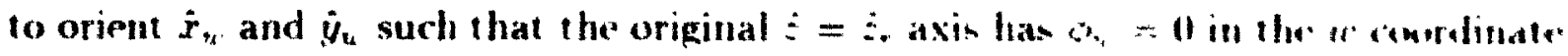
system. Then $\theta=\theta_{u}$. and

$$
\begin{aligned}
& \vec{r} \cdot \vec{p}=r P \cos \theta_{r} \\
& \vec{r} \vec{p}=r P^{2}\left[\sin \theta_{r} \sin \theta_{r} \cos \theta_{r}+\cos \theta \cdot \cos \theta_{n}\right]
\end{aligned}
$$

A wrinkle with this approach is that in Eq. 2.23 ne. also have expressions like of $r$. where the vertors must be expresed in the same courdinate systent. The expremont

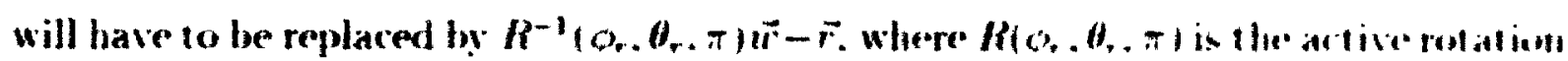

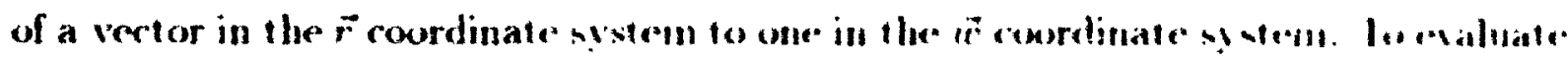

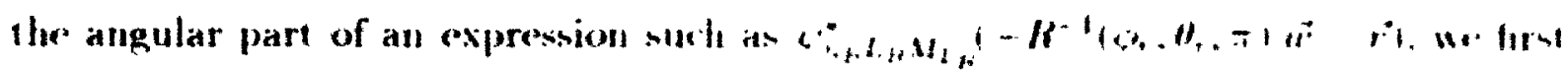
use the following identity for wolid harmonic:

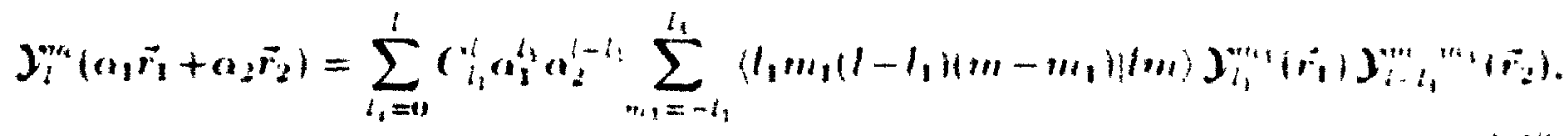

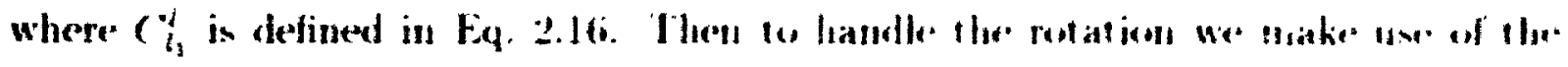
following result

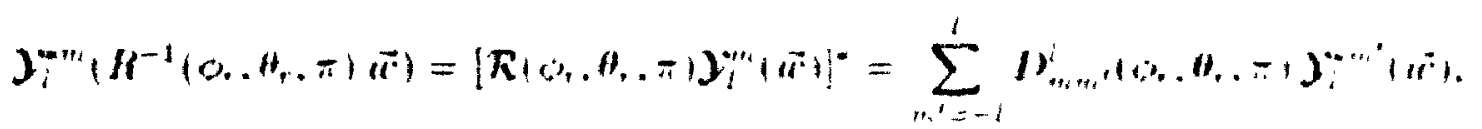

where $V_{m, n}^{l}\left(0_{r}, \theta_{,}, \pi\right)$ is a rotation matrix.

The final wrinkle is that the sulestitution for $\vec{f}$ recpured in the avbastion of

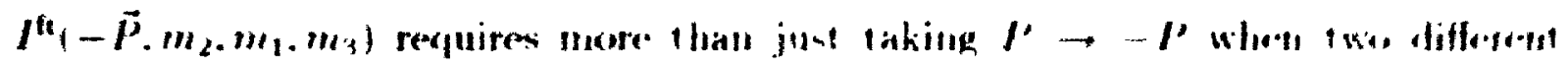

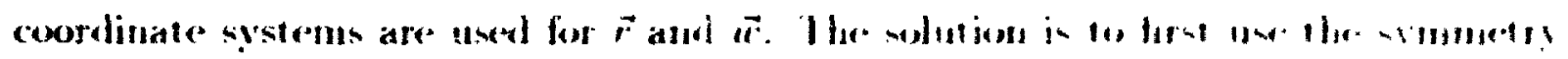


properties of the angular parts of the wavefunctions to obtain

$$
I^{\prime 2}\left(-\vec{P}, m_{2}, m_{1}, m_{3}\right)=(-1)^{3+L_{A}+L_{B}+L_{C}} I^{f t}\left(\vec{P}, m_{2}, m_{1}, m_{3}\right)
$$

after which our particular choice for the $\vec{u}$ coordinate system can be used to evaluate the dot products. At this stage. the expression for the partial wave amplitudes is (before applying Eqs. 2.26 and 2.2i)

$$
\begin{aligned}
& M^{S L}(P)=\gamma_{0} \frac{\sqrt{32 \pi(2 L+1) E_{A} E_{B} E_{C}}}{2 J_{A}+1} \sum_{\substack{M_{L_{A}}, M_{S_{A}}, M_{L_{B}}, M_{S_{B}}, M_{S_{B}} \\
M_{L_{C}}, M_{S_{C}}, M_{C_{C}, m}}} \\
& \left.\times\left\langle\operatorname{LOS}\left(M_{J_{B}}+M_{J_{C}}\right)\right\} J_{A}\left(M_{J_{B}}+M_{J_{C}}\right)\right\rangle\left\langle J_{B} M_{J_{B}} J_{C} M_{J_{C}} \mid S\left(M_{J_{B}}+M_{J_{C}}\right)\right\rangle \\
& \times\left\langle L_{A} M_{L_{A}} S_{A}, M_{S_{A}} \mid J_{A}\left(M_{J_{B}}+M_{J_{C}}\right)\right\rangle\left\langle L_{B} M_{L_{B}} S_{B} M_{S_{B}} \mid J_{B} \cdot M_{J_{B}}\right\rangle
\end{aligned}
$$

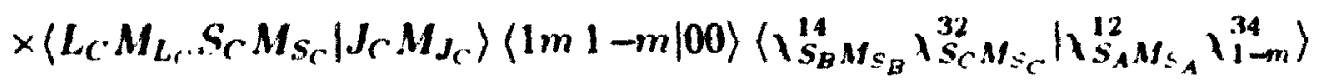

$$
\begin{aligned}
& \times\left[\left\langle\phi_{B}^{14} \phi_{C}^{32} \mid \phi_{A}^{12} \phi_{0}^{34}\right\rangle I^{\prime t}\left(P \leq, m_{1}, m_{2}, m_{3}\right)\right. \\
& \left.+(-1)^{L_{A}+L_{B}+L_{C}+S_{A}+S_{B}+S_{C}}\left\langle\phi_{B}^{32} \phi_{C}^{14} \mid \phi_{A}^{12} \phi_{0}^{34}\right\rangle I^{\mathrm{ft}}\left(P \dot{\Sigma}_{,}, m_{2} \cdot m_{1}, m_{3}\right)\right]
\end{aligned}
$$

where

$$
\begin{aligned}
& I^{\prime \prime}\left(P \Sigma . m_{1} \cdot m_{2} \cdot m_{3}\right)=-\frac{8}{(2 \pi)^{\frac{3}{2}}} \int_{0}^{x} \mathrm{~d} r \int_{0}^{\infty} \mathrm{d} u \int_{-1}^{1} \mathrm{~d}\left(\cos \theta_{u}\right) r^{2} u^{2},-\frac{1}{2} b u u_{\min }^{2} \\
& \times \int_{-1}^{1} \mathrm{~d}\left(\cos \theta_{r}\right) \epsilon^{i m_{-} P u^{\prime} \cos \theta_{r} \cos \theta_{u^{\prime}}+i m_{+} P r \cos \theta_{r}}
\end{aligned}
$$

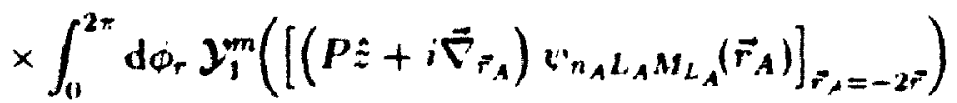

$$
\begin{aligned}
& \times \int_{0}^{2 \pi} \mathrm{d} \phi_{u} \cdot e^{i m-P u} \sin \theta_{r} \sin \theta_{u} \cos \phi_{u} \because_{n_{B} L_{B} M_{L_{B}}}\left(-R^{-1}\left(c_{r} \theta_{r} . \pi\right) \vec{r}-\vec{r}\right) \\
& \times \varphi_{n_{c} \cdot L_{C} \cdot M_{L_{l}}^{*}}\left(K^{-1}\left(O_{r}, \theta_{r}, \pi ; \vec{r}-\vec{r}\right) .\right.
\end{aligned}
$$

Wic can now carry out the $\theta_{r} . O_{r}$ and $o_{u}$ integrations analytically. in principle. In practice. we will only do those for $\phi_{r}$ and $\phi_{u}$. The integral over $o_{u}$. is done by means of 
the residue theorem. The integral over $\phi_{r}$ depends upon the form of the wavefunction of meson .4 - we will again assume that the radial portions of the meson wavefunctions are expressed as linear combinations of the first $N+1$ SHO radial wavefunctions, as in Eq. 2.9. Once this assumption is made, the $o_{r}$ integral dow's not require any special techniques: the inal result after doing it is rather complicated but we give it here for completeness:

$$
\begin{aligned}
& M^{s L}(P)=\int_{0}^{\infty} \mathrm{d} r \int_{0}^{\infty} \mathrm{d} w \int_{0}^{\pi} \mathrm{d} \theta_{r} \int_{0}^{\pi} \mathrm{d} \theta_{u}, g_{1}\left(r, w, \theta_{r}, \theta_{u}\right) \sqrt{2 L+1}
\end{aligned}
$$

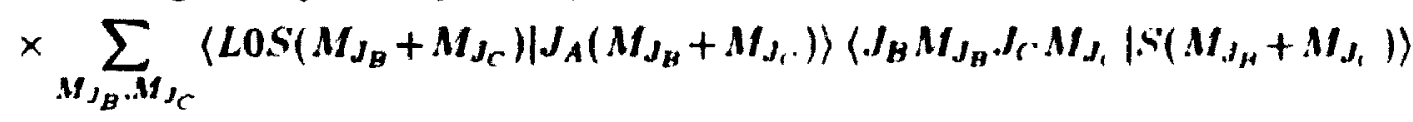

$$
\begin{aligned}
& \times \sum_{M_{L_{A}}, M_{L_{B}}, M_{L_{C}}}\left\langle L_{A} M_{L_{A}} S_{A}\left(M_{J_{B}}+M_{J_{C}}-M_{L_{A}}\right) \mid J_{A}\left(M_{J_{B}}+M_{J_{c}}\right)\right\rangle \\
& \times\left(L_{B} \cdot M_{L_{B}} S_{B}\left(M_{J_{B}}-M_{L_{B}}\right) \mid J_{B} M_{J_{B}}\right)\left(L_{C} \cdot M_{L_{c}} . S_{C} \cdot\left(M_{I_{1}}-M_{L_{1}}\right)\left|J_{C} \cdot M_{J_{1} \cdot}\right\rangle\right. \\
& \times\left\langle 1\left(M_{L_{B}}+M_{L_{C}}-M_{L_{A}}\right) 1\left(M_{L_{A}}-M_{L_{H}}-M_{L_{L_{C}}}\right) \mid 00\right\rangle
\end{aligned}
$$

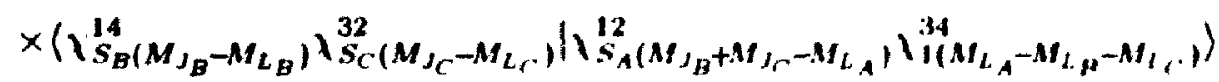

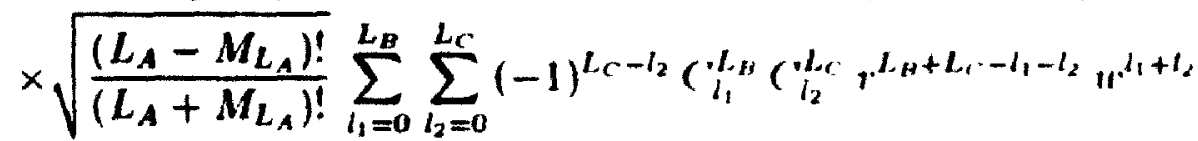

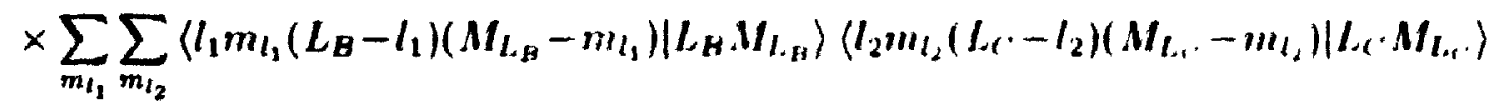

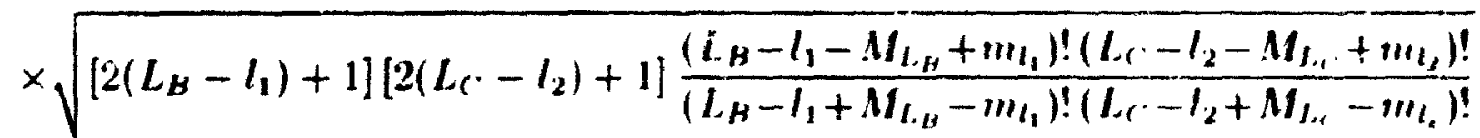

$$
\begin{aligned}
& \times P_{I_{B}-L_{1}}^{M_{L_{B}}-m_{I_{1}}}\left(\cos \theta_{r}\right) P_{L_{C}-l_{2}}^{M_{L_{C}}-m_{i_{2}}}\left(\cos \theta_{r}\right) \\
& \times \sum_{m^{\prime}=-l_{1}}^{l_{1}} \sum_{m^{\prime \prime}=-l_{2}}^{l_{2}} \sqrt{\left(2 l_{1}+1\right)\left(2 l_{2}+1\right) \frac{\left(l_{1}-m^{\prime}\right) !\left(l_{2}-m \prime^{\prime \prime}\right) !}{\left(l_{1}+m^{\prime}\right) !\left(l_{2}+m^{\prime \prime}\right) !}}(-1)^{m^{\prime}+m_{i}^{\prime \prime}}
\end{aligned}
$$

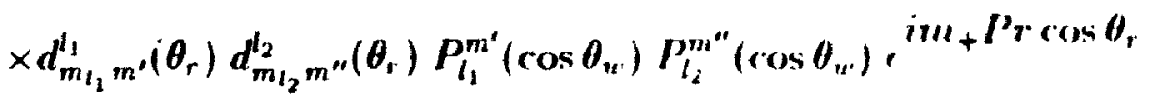

$$
\begin{aligned}
& \times\left[i g_{2}\left(M_{L_{A}} \cdot M_{L_{B}}+M_{L_{C}} \cdot r . \theta_{r}\right)+g_{3}\left(M_{L_{A}} . . H_{L_{H}}+M_{\left.L_{r} . . r, \theta_{r}\right)}\right)\right]
\end{aligned}
$$

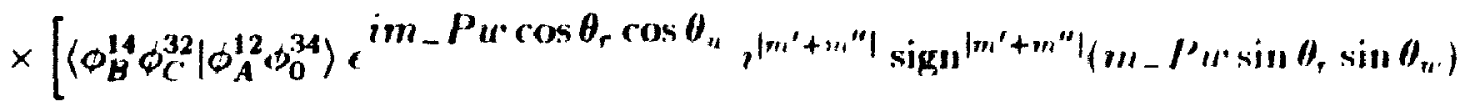

$$
\begin{aligned}
& \times J_{\left|m^{\prime}+m^{\prime \prime}\right|}\left(\left|m-P u \cdot \sin \theta_{r} \sin \theta_{w}\right|\right)
\end{aligned}
$$




$$
\begin{aligned}
& +(-1)^{L_{A}+L_{B}+L_{C}+S_{A}+S_{B}+S_{C}}\left\langle\phi_{B}^{32} \phi_{C}^{14} \mid \phi_{A}^{12} \phi_{0}^{34}\right\rangle e^{-i m_{-} P w \cos \theta_{r} \cos \theta_{u^{\prime}} ;\left|m^{\prime \prime}+m^{\prime \prime}\right|} \\
& \left.\times \operatorname{sign}^{\left|m^{\prime}+m^{\prime \prime}\right|}\left(-m_{-} P w \sin \theta_{r} \sin \theta_{u^{\prime}}\right) J_{\left|m^{\prime}+m^{\prime \prime}\right|}\left(\left|m_{-} P w \sin \theta_{r} \sin \theta_{u^{\prime}}\right|\right)\right]
\end{aligned}
$$

where $d_{m m^{\prime}}^{l}(\theta)$ is related to the rotation matrix by

$$
D_{m m^{\prime}}^{l}(\phi, \theta, \lambda) \equiv \epsilon^{-1 m \phi} d_{m m^{\prime}}^{l}(\theta) \epsilon^{-1 m^{\prime} x}
$$

$P_{l}^{m}(x)$ is an associated Legendre function, $J_{l}(x)$ is a Bessel function of the first kind, and $\operatorname{sign}(x)$ is +1 if $x \geq 0$ and -1 if $x<0$. The $g$ functions are given by

$$
\begin{aligned}
& g_{1}\left(r, u \cdot \theta_{r}, \theta_{w}\right) \equiv \frac{-\sqrt{3}}{2 \pi^{2}} \frac{\sqrt{2 L_{A}+1}}{2 J_{A}+1} \sqrt{E_{A} E_{B} E_{C}}(-1)^{L_{B}} \beta_{A}^{L_{A}+\frac{3}{2}} 3_{B}^{L_{B}+\frac{2}{2}} \beta_{A}^{L_{B}+\frac{3}{2}} \\
& \left.\times r^{2} u^{2} \sin \theta_{r} \sin \theta_{u}\right\urcorner\left(r, w \cdot \theta_{w}\right)(-2 r)^{L_{A}-1} \\
& \times c^{-2 \beta_{A}^{2} r^{2}} c^{-\beta_{B}^{2}\left(r^{2}+u^{2}+2 r u \cos \theta_{u}\right) / 2} c^{-3^{2} \cdot\left(r^{2}+u^{2}-2 r u \cdot \cos \theta_{u}\right) / 2} \\
& \times\left[\sum_{b=0}^{N} d_{b}^{B} \sqrt{\frac{2 b !}{\Gamma\left(b+L_{B}+\frac{3}{2}\right)}} L_{b}^{L_{B}+\frac{1}{2}}\left(\beta_{B}^{2}\left(r^{2}+u^{2}+2 r u \cos \theta_{u}\right)\right)\right] \\
& \times\left[\sum_{c=0}^{N} d_{r}^{c} \sqrt{\frac{2 c !}{\Gamma\left(c+L_{C}+\frac{3}{2}\right)}} L_{c}^{L_{c}+\frac{1}{2}}\left(\beta_{c}^{2}\left(r^{2}+u^{2}-2 r u \cdot \cos \theta_{u}\right)\right)\right], \\
& g_{2}\left(M_{L_{1}}, M_{L_{B}}+M_{L_{C}}, r, \theta_{r}\right) \equiv
\end{aligned}
$$

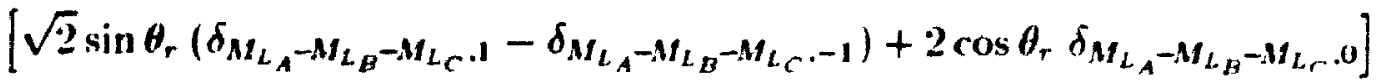

$$
\begin{aligned}
& \times P_{L_{A}}^{M_{L_{A}}}\left(\cos \theta_{r}\right)\left[\sum_{a=0}^{N} a_{a} \sqrt{\frac{2 a !}{\Gamma\left(a+L_{A}+\frac{2}{2}\right)}}\left(\left(L_{A}-43_{A}^{2} r^{2}\right) L_{A}^{L_{A}+\frac{1}{2}}(4 ;\}_{A}^{2} r^{2}\right)\right. \\
& \left.-83_{A}^{2} r^{2}\left\{\begin{array}{ll}
0 . & \text { if } a=0 \\
L_{a-1}^{L_{A}+\frac{1}{2}}\left(43_{A}^{2} r^{2}\right), & \text { if } a \geq 1
\end{array}\right)\right] \\
& +\left\{\left[\sqrt{2} \cos \theta_{r}\left(\delta_{M_{L_{A}}-M_{L_{B}}-M_{L_{C} .}-1}-\delta_{M_{L_{A}}-M_{L_{B}}-M_{L_{C}}-1}\right)-2 \sin \theta_{r} \delta_{M_{L_{A}}-M_{L_{B}}-M_{L_{f}} .00}\right]\right. \\
& \times \frac{1}{\sin \theta_{r}}\left[L_{A} \cos \theta_{r} P_{L_{A}}^{M_{L_{A}}}\left(\cos \theta_{r}\right)-\left(L_{A}+M_{L_{A}}\right) P_{L_{A}-1}^{M_{L_{A}}}\left(\cos \theta_{r}\right)\right] \\
& \left.+\frac{\sqrt{2} M_{L_{A}}}{\sin \theta_{r}}\left(\delta_{M_{L_{A}}-M_{L_{B}}-M_{L_{C}} .1}+\delta_{M_{L_{A}}-M_{L_{B}}-M_{L_{C}}-1}\right) P_{L_{A}}^{M_{L_{A}}}\left(\cos \theta_{r}\right)\right\}
\end{aligned}
$$




$$
\begin{aligned}
& \times\left[\sum_{a=0}^{N} d_{a}^{A} \sqrt{\frac{2 a !}{\Gamma\left(a+L_{A}+\frac{3}{2}\right)}} L_{a}^{L_{A}+\frac{1}{3}}\left(4: 3_{4}^{2} r^{2}\right)\right] .
\end{aligned}
$$

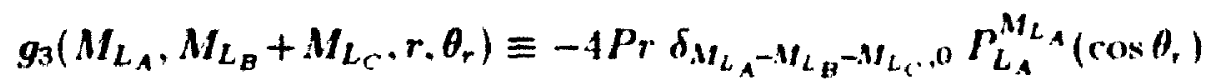

$$
\begin{aligned}
& \times\left[\sum_{a=0}^{N} d_{a}^{A} \sqrt{\frac{2 a !}{\Gamma\left(a+L_{A}+\frac{2}{2}\right)}} L_{a}^{L_{A}+\frac{1}{2}}\left(4: 3_{A}^{2} r^{2}\right)\right] .
\end{aligned}
$$

where $L_{n}^{k}(x)$ is an associated Laguerre polynomial.

We have automated the calculation of partial wave amplitudes with these expressions, using routines written for Mathematica [12] and FORTRAN. In the Mathematica code, an integrand for each partial wave amplitude is prepared symbolically and converted to FORTRAN code. The FORTRAN progran carries ont the integrations numerically using either adaptive Monte (arlo (VEGAS [13]) or a combinalion of adaptive Gaussian quadrature routines. Again. these routines are usable for any' meson decay where the radial portion of the wavefunctions can be expanded in lermis of SHO wavefunctions, and are limited only by the size of the problem and the available. computer resources. The overlaps of the colour, flavour and spin wavefunctions of the mesons and the created pair are calculated using the techniques of Appendix I). 


\section{Chapter 3}

\section{Models of Meson Decay: Two Applications}

\subsection{Setting the Parameters of the Models}

In order to use the models of meson decay introduced in Chapter 2 we must fit the undetermined parameter $\gamma$ ( $\gamma_{0}$ for the flux-tube breaking model). and we must decide which meson space wavefunctions to use. In addition. we must decide which phase space/normalization is best suited to our purposes - we discuss this below.

In this section we consider three types of space wavefunctions. described in some detail in Appendix B.1: SHO wavefunctions with a common oscillator parameter (3). wavefunctions from the relativized quark model (RQM) [4] of Godfrey and Isgur described in Section 1.2.2, and SHO wavefunctions with the effective 3 s $\mathrm{s}$ of Kokoski and Isgur [5]. The RQM wavefunctions and SHO wavefunctions with effective is"s are fully determined and have no parameters left to choose. For the SHO wavefunctions with a common $\beta$. we take $\beta=400 \mathrm{MeV}$. the value used by liokoski and Isgur which lies safely within the range of the effective values fitted by them for the vatious mesons. We also perform another fit of both, and $\beta$ simultaneously: 
We must also decide how to normalize the mock meson wavefunctions and what kind of phase space to use to calculate the decay widths [14]. We investigate three choices. In Chapter 2 we normalized the mock meson wavefunctions relativistically to $2 E$ (Eq. 2.2) and used relativistic phase space (leading to Eq. 2.5), which leads to a factor of $E_{B} E_{C} / M_{A}$ in the final expression for a decay width in the (M frame ( $E$ ) is energy, $M$ is mass). We will refer to this as relativistic phase space/normalization (RPSN). One could also argue that since our decay models are primarily nonrelativistic, it makes sense to use a nonrelativistic normalization and nonrelativistic phase space, which would replace the factor by $M_{B} M_{C} / M_{A}$. We will refer to this as nonrelativistic phase space/normalization (NRPSN). However, there are als) arguments [15] that heavy quark effective theory fixes the assumptions in the mock meson prescription and suggests that the factor be replaced by $\bar{M}_{B} \bar{M}_{C} \cdot / \bar{M}_{A}$, where the $\bar{M}_{\text {, are }}$ the calculated masses of the meson $i$ in a spin-independent quark-ant iquark potential [5]." We will refer to this as the Kokoski-Isgur phase space/normalization (KIISN).

Some comments about the details of the calculations made in this chapter arc in order. For both types of SHO wavefunctions we use quark masses in the ratio $m_{u}: m_{d}: m_{s}=3: 3: 5$ - this differs from the ralculations of kokoski and lsgur [5], which ignored the strange-quark mass difference. For the HQM wavefunctions the quark masses have already been fitted: $m_{u}=220 \mathrm{M} \cdot \mathrm{V}, m_{d}=220 \mathrm{M} \cdot \mathrm{V}$, and $m_{s}=419 \mathrm{MeV}$. We treat all mesons as narrow resonances. Meson masses are takell from the Review of Particle Properties 1994 [1] if the state is included in their Messm Summary Table. If it is not. then the masses predicted in Ref. [1] atre usiel. This includes the masses of the $1^{3} F_{2}, 1^{3} F_{3}$ and $1^{3} F_{4}$ s.s mesons: $2240 \mathrm{M} \cdot \mathrm{V} \cdot$ " $230 \mathrm{M} \cdot \mathrm{V}$ and $2200 \mathrm{MeV}$ respectively. We ignore mass differences between members of the same

\footnotetext{
'In other words $\widetilde{M}_{1}$ is given by the hyperfine averaged mass that is equal to the critl re of gravity of the triplet and singlet masses of a meson multiplet of given orbit al angular momintum $l$. IIhe. main effect of KIPSN is that the widths of decays with pseudosralars in the final st ite arr Ineruased

${ }^{2}$ The one exception was the $1^{3} P_{0}$ sis state ser Tatsle 3.7 .
} 
isospin multiplet ${ }^{3}$ and average over the quoted masses. if different. Meson flavour wavefunctions are also taken from Ref. [4], and are summarized in Appendix B.24.

In order to evaluate the above wavefunctions and phase space/normalization schemes and to fit the value of $\gamma\left(\gamma_{0}\right)$ we carried out a series of least squares fits of the model predictions to the decay widths of 28 of the best known ${ }^{5}$ meson decays: we minimized the quantity defined by $\lambda^{2}=\sum_{1}\left(\Gamma_{1}^{\text {model }}-\Gamma_{t}^{e x p}\right)^{2} / \sigma_{\Gamma_{1}}^{2}$, where $\sigma_{\Gamma_{1}}$ is the experimental error. ${ }^{6}$ The 28 decays used, and the experimental values of their widths [1], are shown in Table 3.1.

The ratios of the model predictions to the experimental values for the widths of the 28 decays are plotted in Figures 3.1 to 3.5 , on logarithmic scales. The fit ted values of $\gamma\left(\gamma_{0}\right)$ and the resulting $\gamma^{2}$ per degree of freedom (dof) are listed in Table 3.2. We do not quote errors on the fitted values of $\gamma\left(\gamma_{0}\right)$ because they are negligible (being at most a change in the last digit quoted) compared to the inherent uncertainties of the models. We have performed fits for the ${ }^{3} P_{0}$ model with both types of SHO wavefunctions, and for the flux-tube breaking model with all three types of wavefunctions. We did not use the RQM wavefunctions with the ${ }^{3} P_{0}$ model because the large number (up to 13) of radial SHO wavefunctions in the linear combination (see Eq. 2.9) exceeds the capacity of our symbolic routines on the atailable computer hardware ${ }^{-}$. Because the results of the ${ }^{3} P_{0}$ and flux-tube breaking models appear to be very similar. we

\footnotetext{
The one exception was for the decay $o \rightarrow k^{+} k^{-}$where the charged and neutral kaon mass difference is significant to the phase space.

${ }^{4}$ For the $\eta$ and $\eta^{\prime}$ we assumed perfert mixing. corresponding $10 \theta_{P}=-9.7^{\circ}$. but we also considered the effert that using $\theta_{p}=-20^{\circ}$ would have on the results of Tables 3.5 through 3.9 . Wi found that while individual deray widths varied by up to a factor of approximately 2 , the changes to the total widths were negligible compared to the of her uncertaint jes of the ralculations.

${ }^{5}$ Wr also required that the decays we selected not involve any particles that undergo significant mixing (e.g. $\left.\eta, \eta^{\prime}\right)$ in order to minimize uncert aint ies in the calculation.

"For the calculations in the flux-tube breaking model, a $1 \%$ error due to the numerical integration was added in quadrature with the experiment al error.

This problem arises for the ${ }^{3} P_{11}$ niodel routines. which are symbolic (and hence are slower and require more memory). but not for the flux-tube breaking model routines. where the brunt of the work is done numerically.
} 


\begin{tabular}{|c|c|c|c|c|c|c|c|}
\hline \multirow{4}{*}{ Decay } & \multirow{4}{*}{$\begin{array}{c}\text { (Mel) } \\
\text { from } \\
\text { Experiment }\end{array}$} & \multicolumn{6}{|c|}{ T (Mel) from Models of Meson Decary } \\
\hline & & \multirow{2}{*}{\multicolumn{2}{|c|}{$\frac{{ }^{3} P_{\mathrm{a}}}{\mathrm{SHO}}$}} & \multicolumn{4}{|c|}{ Flux-tube $13 r e a k i n g$} \\
\hline & & & & \multicolumn{2}{|c|}{ SHO } & \multicolumn{2}{|c|}{ RQMI } \\
\hline & & RPSN & KIPSN & RPSN & KIPSN & RPSN & KII'SN \\
\hline$\rho \rightarrow \pi \pi$ & $151.2 \pm 1.2$ & 96 & 148 & $\overline{93}$ & 1.48 & 10.1 & 152 \\
\hline$b_{1}(1235) \rightarrow \sim \pi$ & $142 \pm 8$ & 176 & 115 & 155 & 104 & औ) & $1: M$ \\
\hline$a_{2}(1320) \rightarrow \rho \pi$ & $75.0 \pm 4.5$ & 6.5 & 38 & 67 & .10 & 8.1 & $16 i$ \\
\hline$a_{2}(1320) \rightarrow K h$ & $5.2 \pm 0.9$ & 11 & 8.0 & 11 & 8.5 & 7.3 & 5.11 \\
\hline$\pi_{2}(1670) \rightarrow f_{2}(1270) \pi$ & $135 \pm 11$ & $14 i$ & 116 & 143 & 117 & ז':3 & 246 \\
\hline$\pi_{2}(1670) \rightarrow \rho \pi$ & $74 \pm 11$ & 232 & 7.1 & 226 & 7.1 & $: 123$ & 9 \\
\hline$\pi_{2}(1670) \rightarrow h^{*}(892) h^{\prime}$ & $10.1 \pm 3.4$ & 38 & 17 & 37 & 17 & .19 & 21 \\
\hline$\rho_{3}(1690) \rightarrow \pi \pi$ & $50.7 \pm 5.5$ & 116 & 35 & $1: 2 \cdot 2$ & אי & lis & 19 \\
\hline$\rho_{3}(1690) \rightarrow \pi$ & $34 \pm 13$ & 36 & 11 & 39 & $1: 1$ & 15 & $1: 3$ \\
\hline$\rho_{3}(1690) \rightarrow K K$ & $3.4 \pm 0.6$ & 9.2 & 3.8 & 9.7 & 1.2 & 1.2 & 1.7 \\
\hline$f_{2}(12 \pi 0) \rightarrow \pi \pi$ & $156.8 \pm 3.2$ & $20: 3$ & 109 & 209 & IIt & $15 \%$ & Sil \\
\hline $\int_{2}(1270) \rightarrow K K$ & $8.6 \pm 0.8$ & 7.2 & 5.4 & 7.1 & 5.7 & 5.0 & 3.5 \\
\hline$f_{4}(2050) \rightarrow$ uiv & $54 \pm 13$ & 53 & 11 & 5.4 & 11 & 91 & is \\
\hline$f_{4}(20.50) \rightarrow \pi \pi$ & $35.4 \pm 3.8$ & $12: 3$ & 25 & 132 & $2 x$ & א & 11 \\
\hline$f_{4}(2050) \rightarrow K \dot{K}$ & $1.4 \pm 0.7$ & 5.1 & 1.6 & $5 . x$ & 1.7 & 1.8 & 0.5 \\
\hline$o \rightarrow h^{+} h^{-}$ & $2.17 \pm 0.05$ & 2.37 & 2.83 & 2.28 & 2.80 & 2.80 & $2 .(6)$ \\
\hline$f_{2}^{\prime}(1525) \rightarrow h \bar{h}$ & (i) \pm 5 & 117 & 61 & IIs & (i.1 & $! N$ & $1 !$ \\
\hline$h^{-}(892) \rightarrow h \pi$ & $50.2 \pm 0.5$ & 36 & 53 & 34 & 政 & $3 x$ & 72 \\
\hline$h_{0}^{*}(1430) \rightarrow h \pi$ & $267 \pm 36$ & $16: 3$ & 8.1 & 117 & (ii) & $x+5$ & $1: 31$ \\
\hline$K_{2}(1430) \rightarrow K \pi$ & $48.9 \pm 1.7$ & $10 \mathrm{~s}$ & 56 & 112 & (il) & s & $1: 3$ \\
\hline$K_{2}(1430) \rightarrow h^{*}(892) \pi$ & $2.4 .8 \pm 1.7$ & 27 & 16 & 27 & 17 & 31 & IX \\
\hline$K_{2}^{*}(1430) \rightarrow K \rho$ & $8.7 \pm 0.8$ & 9.3 & 1.9 & 9.6 & $\pi$ & 12 & $5 . x$ \\
\hline$K_{2}(1430) \rightarrow h_{-}$ & $2.9 \pm 0.8$ & 2.6 & 1.1 & 2.6 & 1.1 & $1: 2$ & I. \\
\hline$h_{3}(1780) \rightarrow h_{p}$ & $7.1 \pm 10$ & 2.1 & $\overline{T .7}$ & 25 & S.1 & $2 x$ & $\therefore .7$ \\
\hline$h_{3}(1780) \rightarrow h^{*}(892) \pi$ & $45 \pm 7$ & $83: 3$ & 11 & 34 & 12 & 37 & 12 \\
\hline$h_{3}(1780) \rightarrow h \pi$ & $31.7 \pm 3.7$ & 87 & $2 x$ & $9: 2$ & 301 & 5 & 16 \\
\hline$h_{4}(20.45) \rightarrow h \pi$ & $19.6 \pm 3.8$ & 55 & 13 & 59 & 11 & $2 x$ & (i.2 \\
\hline$h_{4}^{*}(2045) \rightarrow h^{*}(892) 0$ & $2.8 \pm 1.1$ & 3.2 & 1.0 & 3.3 & 1.1 & 1.7 & 1.1 \\
\hline
\end{tabular}

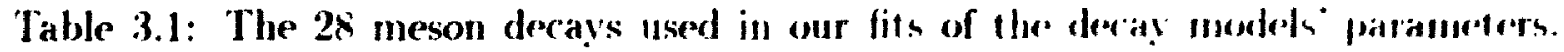
The experimental widths are shown. as are the model result o for six combinat iome of decay model. wavefunctions. and phase spare/normalization (specified in the swomel. third and fourth rows of the leading. respeetively). 


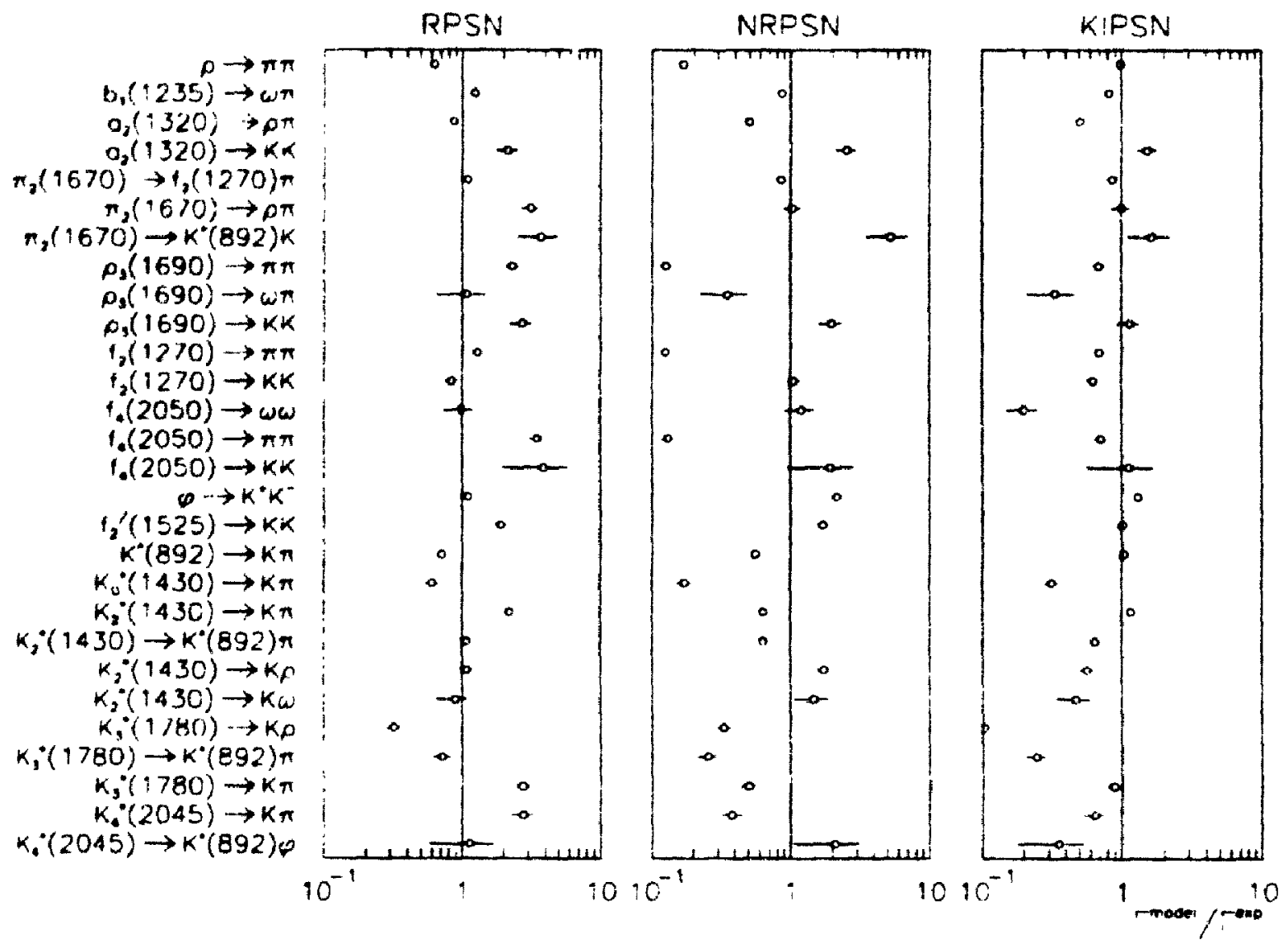

Figure 3.1: Ratios of the predictions of the ${ }^{3} P_{0}$ model of meson decay to the experimental values, for the widths of the 28 decays used in the fits. In this figure SHO wavefunctions with a common 3 were used. The three graphs use different phase space/normalization schemes.

chose not to write a numerical version of the ${ }^{3} P_{0}$ routines to enable us to use the RQM wavefunctions. For each decay model and wavefunction combination. we tried all three phase space/normalization schemes.

When examining Figures 3.1 to 3.5. note that if a particular model described the dat a exactly. all the points for it would hie along the vertical solid line at $\Gamma^{\text {model }} / \Gamma^{\text {exp }}=1$. Hecause $\gamma\left(\gamma_{0}\right)$ is an overall factor in the amplitude. changing it would move all of the points to the left or right by the same amount. Some distributions appear lopsided about $\Gamma^{\text {mudel }} / \Gamma^{\text {exp }}=1$, because the experiment ally well known decays dominate the 


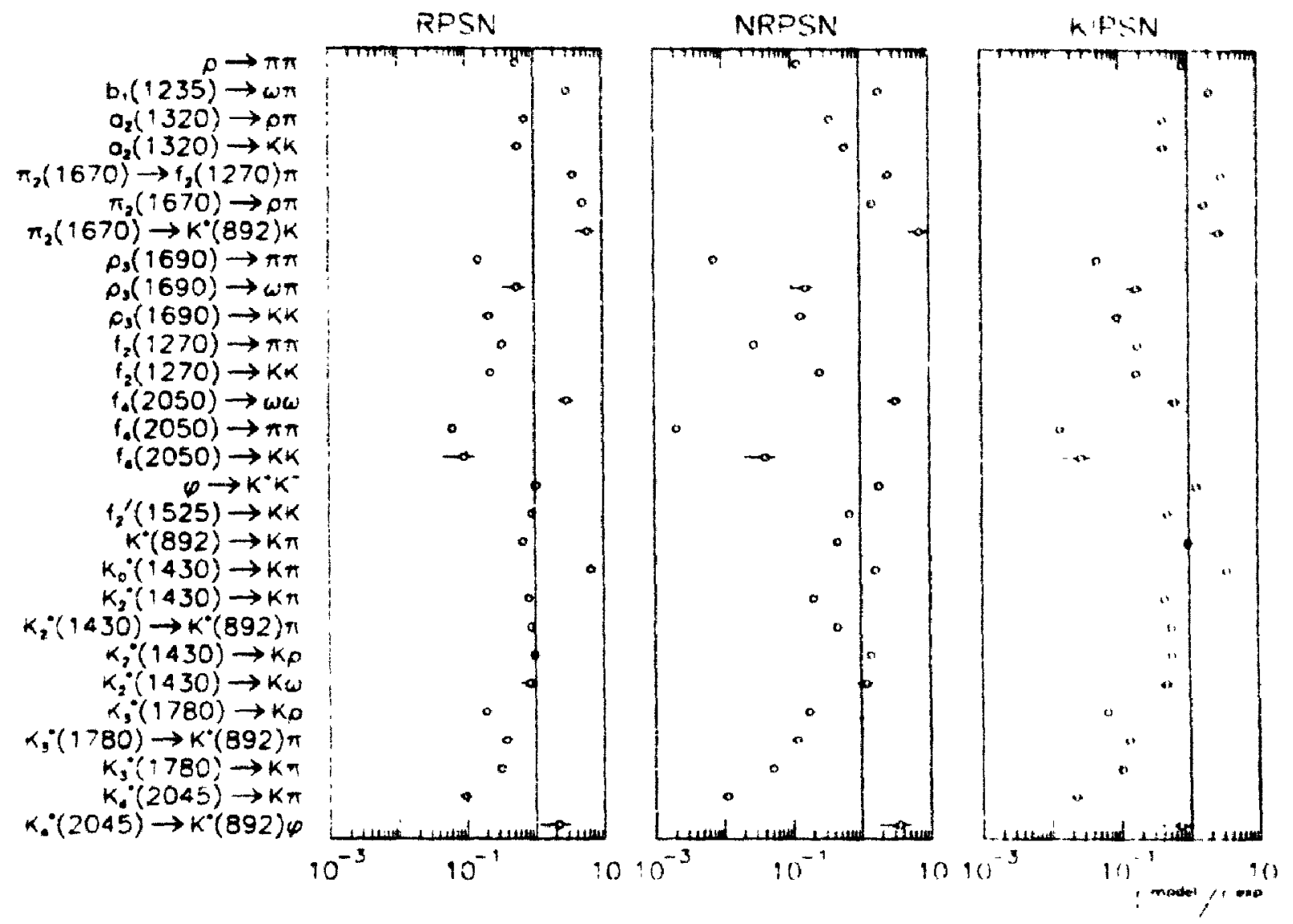

Figure 3.2: Ratios of the predictions of the ${ }^{3} P_{0}$ model of meson deray to the experimental values, for the widths of the $2 x$ decays used in the fits. In this figure SHO wavefuactions with effective is's were used. The three graphs use dilferent phase. space/nurmalization schemes.

fit.

The first thing to notice from the figures and the $1^{2}$ values of Talse 3.2 is that the results with NRPSN are significantly worse than the ones using the of her two chesices of phase space/normalization. We will hence not Use NRPSN for conr calculations. Similarly, the SHO wavefunctions with effective is"s do worse that cither of the of her two types of wavefunctions. and we will not use them either. That leaves us with six different combinations of decay model. wavefunction. and phase space/mormalization - these are the combinations given in Table 3.1. Some general comments are in order. 


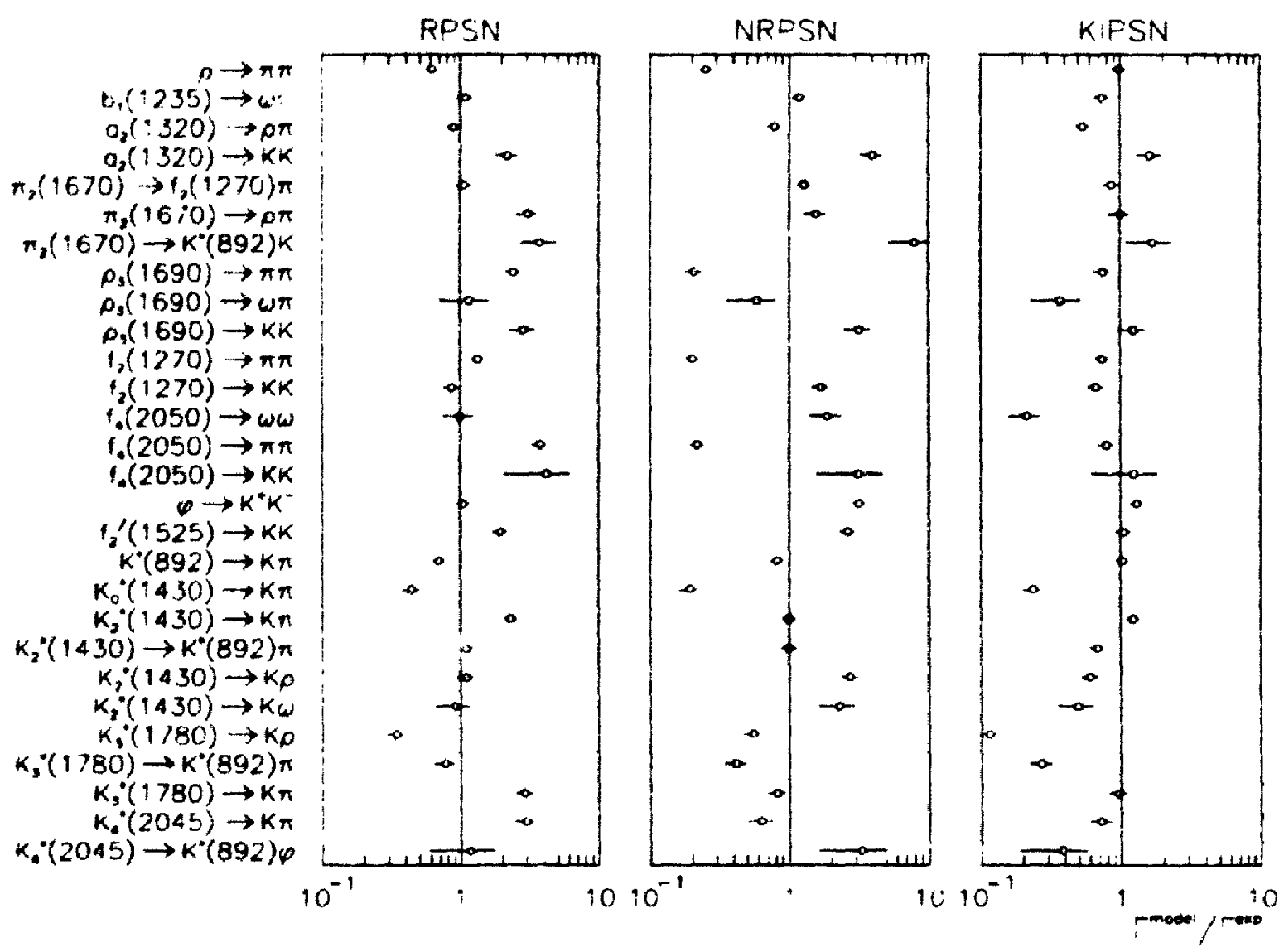

Figure 3.3: Ratios of the predictions of the fux-tube breaking model of meson decay to the experimental values, for the widths of the 28 decays used in the fits. In this figure SIIO wavefunctions with a imon 3 were used. The three graphs use different. phase' space/normalization schem s.

First. the $1^{2}$ values of Table 3.2 make it clear that these models are not very accurat $a^{*}$ - the best they can hope for is to predict a decay width to within a factor of 2. and even larger deviations are common. Since they are coarse models of a complicated theory. this is not surprising. In addition, the six rombinations can give rather different results. We will use this to our advantage by taking the spread in the predictions of the different combinations as a rough guide to the trust worthiness of

\footnotetext{
"It should be notec that past practice has often been to look for agreenent in the amplitudes. rather than the widths. which tends to make the sit uation look better.
} 


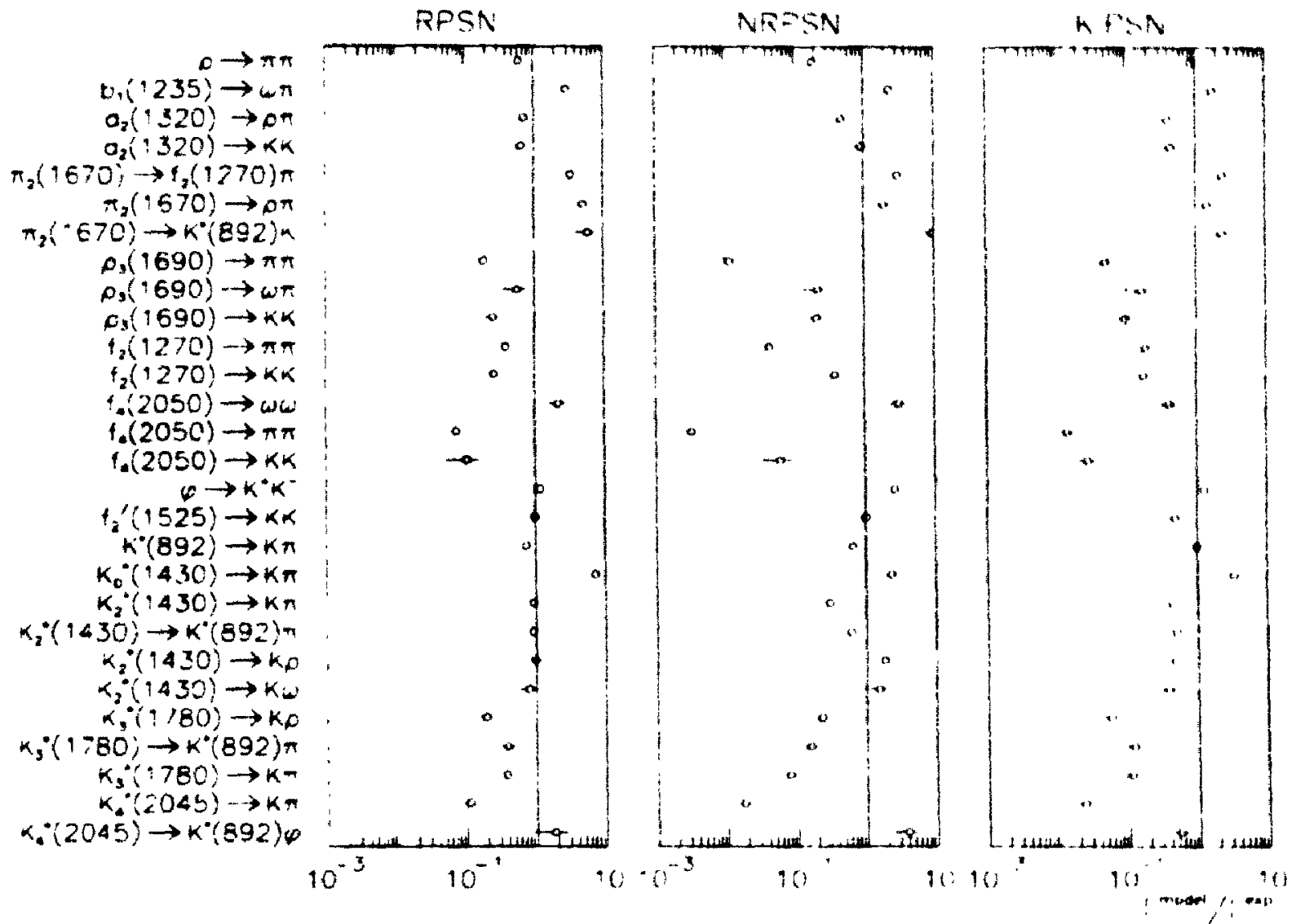

Figure 3.4: Ratios of the predictions of the flux-tule breaking medel of mescon deray to the experimental values, for the widths of the 28 decays used in the lits. In this figure SHO wavefunctions with effective is were used. Ther three graphis use differest phase space/normalization schemes.

the calculations.

From Table 3.1 and Figures 3.: through 3.5 one call see that the result for the. ${ }^{3} P_{0}$ and flux-tube breaking models for the SHO wavefunctions are very silnilar, an previously noted. ${ }^{9}$ This is in agreement with the cobservations of kokonki and lagur

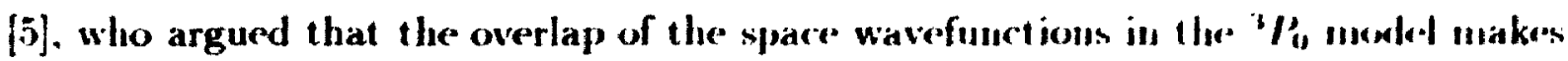
it unlikely that the decay will proceed if the quark-antiquark pair in created fat from

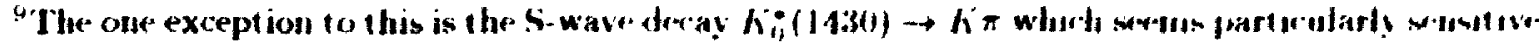
to thr noodel.
} 


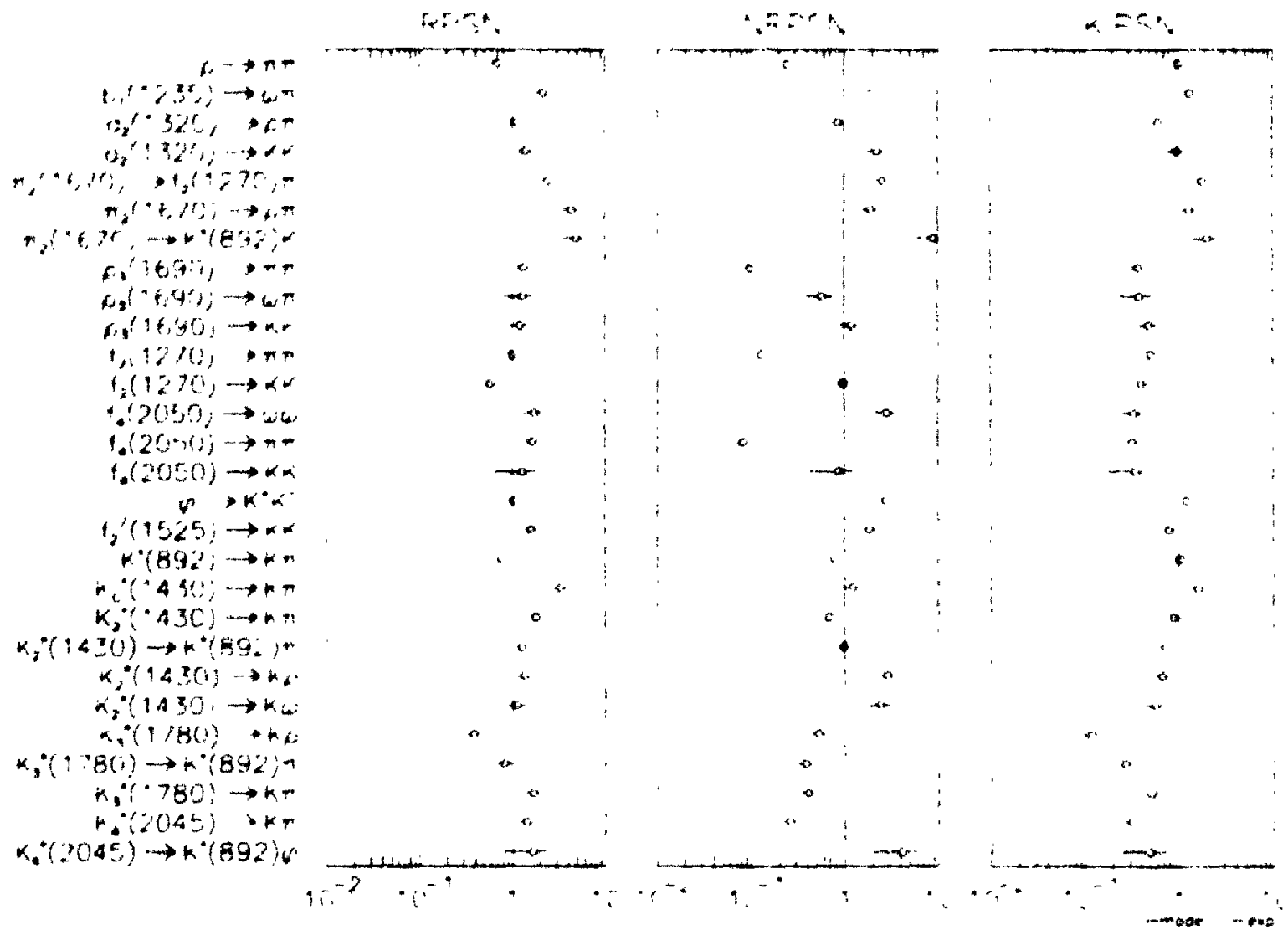

Figure 3.5: Katios of the predictions of the flux-tube breaking moded of meson deact to the experimemal values. for the nidthe of the $2 x$ decays used in the fits. In this ligure HQM Natefunctions were used. The thres graphs no different phase space/mormalization schenes.

a straght lime connecting the two mesons. This would have the same effect as the functional form of $?(\vec{r}, \overrightarrow{\vec{r}})$ in the flux-tube breaking model. Despite the similarity in the individual decays. the $y^{2}$ values of the fits are better for the Hux-tube breaking mokel. indicating at least some preference for this model.

In comparing the SHO wavefunctions ( we will henceforth only speak of the SHO) wavefunctions with a common ti to the RQM Wavefunctions. We note that the RQM

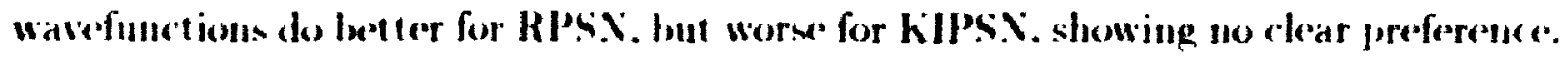

KIll'S. gives letter owerall tit- to the data than KPSS. However. certain derays. 


\begin{tabular}{|c|c|c|c|c|c|}
\hline $\begin{array}{l}\text { Meson Decay } \\
\text { Model }\end{array}$ & Tlavefunctions & $\begin{array}{l}\text { Phase Space: } \\
\text { Normalization }\end{array}$ & .7 & $\sqrt{9}, 1$ & $1^{2}$ ilue \\
\hline$P_{0}$ & SHO (common 3 ) & $\begin{array}{l}\text { RISS } \\
\text { IRI'sx } \\
\text { kll'sx }\end{array}$ & IIM) Ma. & $\begin{array}{l}19.7 \\
11.1 \\
6.2 .1\end{array}$ & $\begin{array}{l}0.1 \\
11.1 \\
2(1.9)\end{array}$ \\
\hline$\because P_{0}$ & Sillo (effective fs) & 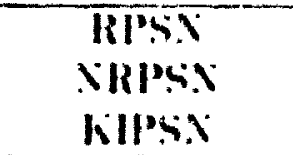 & varie: & $\begin{array}{l}17.6 \\
23.5 \\
11.1\end{array}$ & $\begin{array}{l}110 \\
7616 \\
161\end{array}$ \\
\hline $\begin{array}{l}\text { Flux-1ube } \\
\text { Breaking }\end{array}$ & SHO (rommon il) & $\begin{array}{l}\text { IRl'Sx } \\
\text { NRPSA } \\
\text { hllses }\end{array}$ & f(B) $\mathrm{M} \cdot \mathrm{I}$ & $\begin{array}{l}\text { T(i.1) } \\
20.7 \\
10.1\end{array}$ & $\begin{array}{l}1 \times 5 \\
\vdots 16 \\
3.11\end{array}$ \\
\hline $\begin{array}{l}\text { Flux-1 uhe } \\
\text { Breaking }\end{array}$ & SHO (ellectivex fls & $\begin{array}{l}\text { RPSX } \\
\text { IRPSix } \\
\text { KIPSA }\end{array}$ & vatrien & $\begin{array}{l}2.11 \\
211.1 \\
11.6\end{array}$ & $\begin{array}{l}271 \\
\text { intiin } \\
\text { lini }\end{array}$ \\
\hline $\begin{array}{l}\text { Flux-tuhe } \\
\text { Bronking }\end{array}$ & RQMI & $\begin{array}{l}\text { RI'SX } \\
\text { NII'SX } \\
\text { hll's. }\end{array}$ & marien & $\begin{array}{l}211.5 \\
31.1 \\
13 .\end{array}$ & $\begin{array}{l}12 x \\
110.3\end{array}$ \\
\hline$" H$ & SHO (commons st & $\begin{array}{l}\text { RPSK } \\
\text { NHISX } \\
\text { hIPSX }\end{array}$ & $\begin{array}{l}1.1 \mathrm{M} .1 \\
1.1 \mathrm{M} M .1 \\
371 \mathrm{M} .1\end{array}$ & $\begin{array}{l}13.1 \\
16.5 \\
\text { i.tii) }\end{array}$ & $\begin{array}{l}\text { lin } \\
\text { linti } \\
21.11\end{array}$ \\
\hline
\end{tabular}

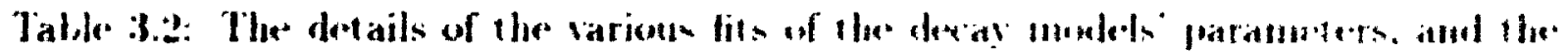

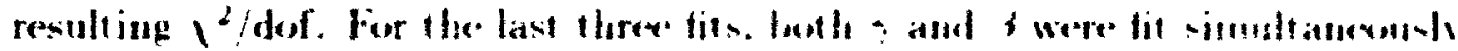

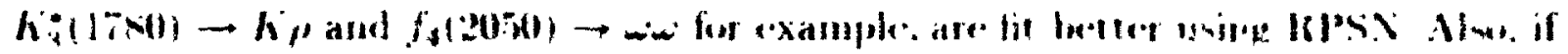

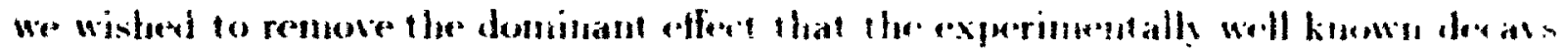

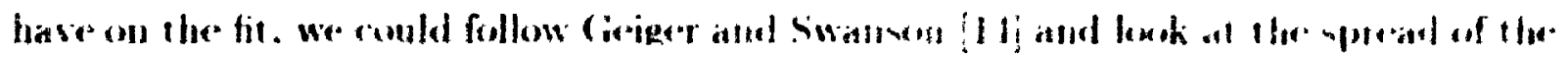

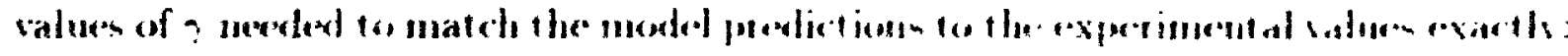

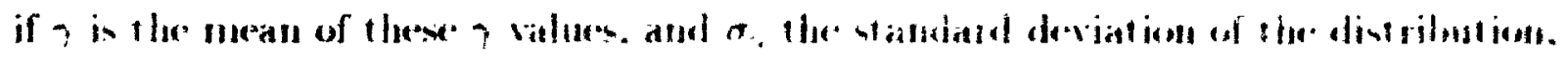

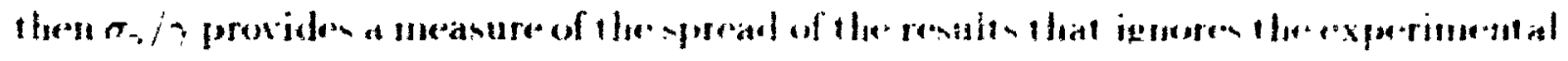

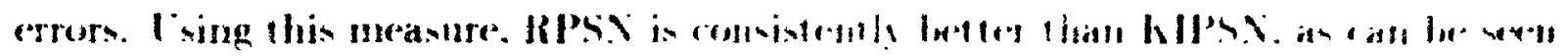
in Tahlo :3.3.

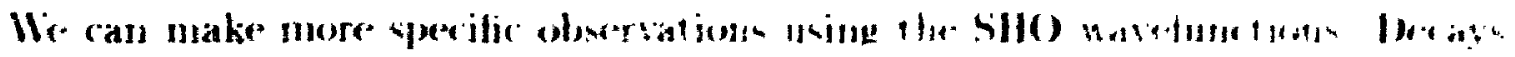

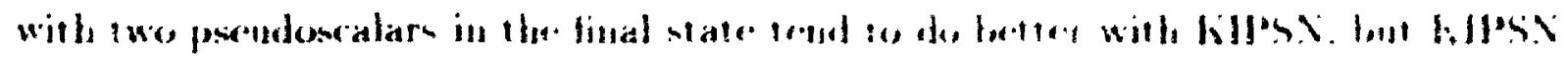

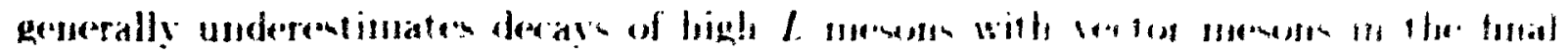




\begin{tabular}{|c|c|c|c|c|c|c|}
\hline $\begin{array}{c}\text { Meson Decay } \\
\text { Model }\end{array}$ & Wavefunctions & $\begin{array}{l}\text { Phase Space' } \\
\text { Normalization } \\
\end{array}$ & $\begin{array}{c}\text { i3 } \\
\text { (MeV) }\end{array}$ & $\bar{i}$ & $\sigma_{n}$ & $\bar{\sigma} / \bar{\gamma}$ \\
\hline $3 P_{0}$ & SIIO (common $\beta)$ & $\begin{array}{l}\text { RPSN } \\
\text { NRPSN } \\
\text { KIPSN }\end{array}$ & 400 & $\begin{array}{l}8.73 \\
19.3 \\
8.17\end{array}$ & $\begin{array}{l}2.84 \\
10.1 \\
3.15\end{array}$ & $\begin{array}{l}0.325 \\
0.52 \% \\
0.386\end{array}$ \\
\hline${ }^{3} P_{0}$ & SHO (effective $B$ s) & $\begin{array}{c}\text { RPSN } \\
\text { NRPSN } \\
\text { KIPSN }\end{array}$ & varies & $\begin{array}{l}25.7 \\
76.4 \\
26.5\end{array}$ & $\begin{array}{l}16.3 \\
108 \\
23.0\end{array}$ & $\begin{array}{c}0.634 \\
1.41 \\
0.868\end{array}$ \\
\hline $\begin{array}{l}\text { Flux-tube } \\
\text { Breaking }\end{array}$ & SHO (common $\beta$ ) & $\begin{array}{c}\text { RPSN } \\
\text { NRPSN } \\
\text { KIPSN }\end{array}$ & 400 & $\begin{array}{l}14.3 \\
31.6 \\
13.4\end{array}$ & $\begin{array}{l}4.86 \\
16.9 \\
5.13\end{array}$ & $\begin{array}{l}0.339 \\
0.537 \\
0.384\end{array}$ \\
\hline $\begin{array}{l}\text { Flux-tube } \\
\text { Brraking }\end{array}$ & SHO (effective $\beta^{\circ} s$ ) & $\begin{array}{c}\text { RPSN } \\
\text { NRPSN } \\
\text { KIPSN }\end{array}$ & varies & $\begin{array}{l}31.0 \\
90.5 \\
31.9\end{array}$ & $\begin{array}{l}18.7 \\
124 \\
26.6\end{array}$ & $\begin{array}{c}0.604 \\
1.37 \\
0.834\end{array}$ \\
\hline $\begin{array}{l}\text { Flux-tube } \\
\text { Breaking }\end{array}$ & RQM & $\begin{array}{l}\text { RPSN } \\
\text { NRPSN } \\
\text { KIPSN }\end{array}$ & varies & $\begin{array}{l}18.1 \\
42.1 \\
17.1\end{array}$ & $\begin{array}{l}5.02 \\
27.6 \\
6.01\end{array}$ & $\begin{array}{l}0.278 \\
0.656 \\
0.351\end{array}$ \\
\hline
\end{tabular}

Table 3.3: The valuc of $;, \sigma_{2}$ and $\sigma_{2} / \gamma$ for the 28 decay's used in the fits. where the ( $\left.q_{1}\right)$ value for a decay matches the model prediction to the experimental value. Thus $\sigma_{\sqrt{ }} / \gamma$ provides a measure of the spread of the results that ignores the experimental errors.

state On the other hand RPSN tends to overestimate decays with iwo pseudoscalars in the final state. Trends can also be observed with the flux-tube breaking model using the RQM wavefunctions. Haring said all this we st ress that these are only general olsservations and exceptions can be found to any of them in Table 3.1. One must therefore be very careful not to take any predictions of these models at face value but should try if possible to compare the predicted decay to a similar one that is experimentally well known.

Finally. we consider the sensitivity of our results to 6 . which we must choose for the SHO wavefunctions. Different quark models predict different values of is, so there is no dear theoretical prediction to draw upon. It sliould be noted that although meson decay widths can depend strongly on the value of 3 . to some extent Wre aileviate this effect because we fit ? to experimental data after selecting a value 


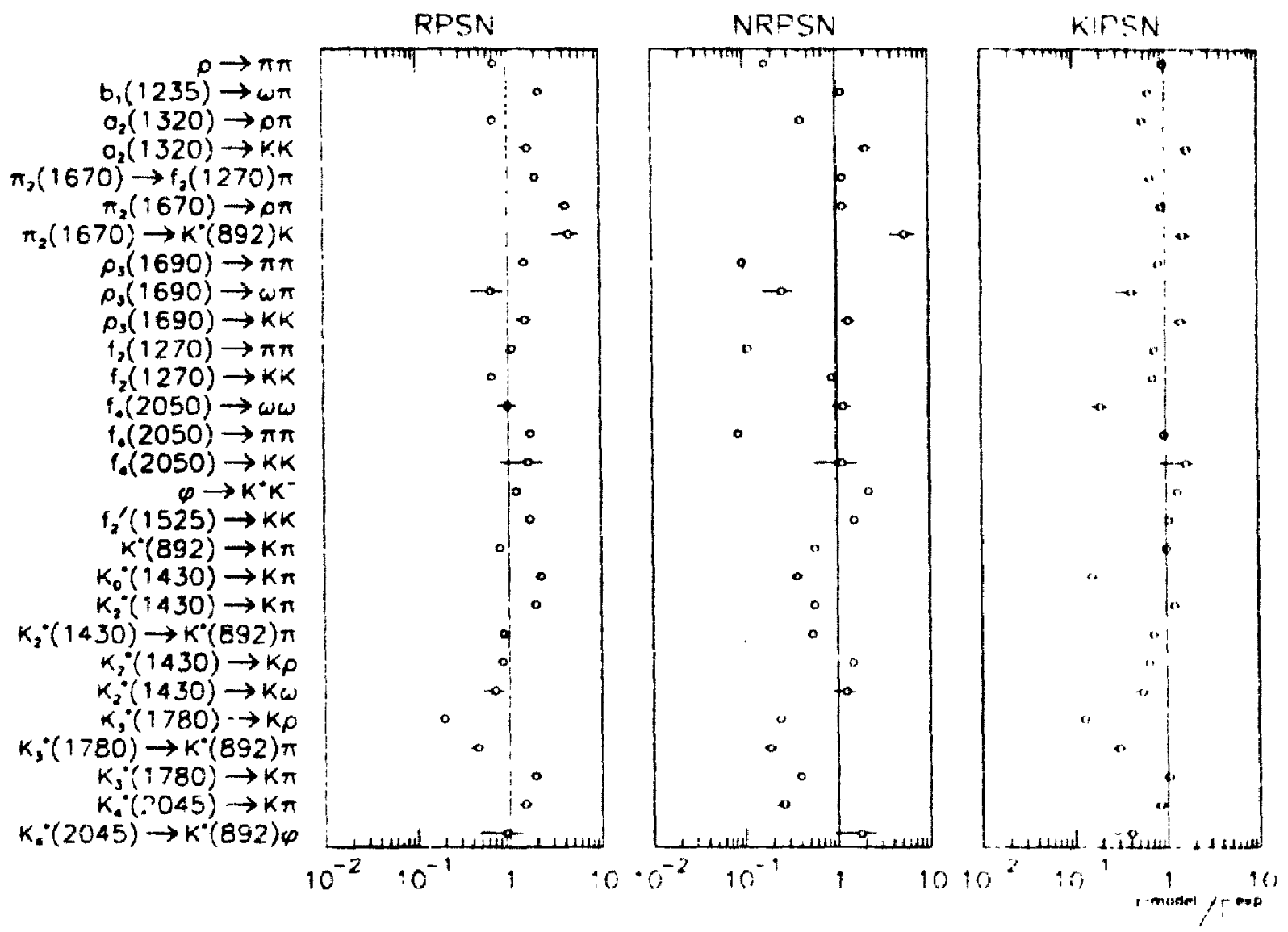

Figure 3.6: Ratios of the predictions of the " $P_{0} \cdot$ m.del of mescii deray te the experimental values, for the widths of the 28 decays used in the lits. In this figure Sillo

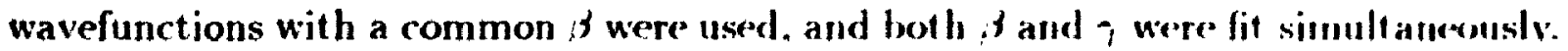
The three graphs use different phase space/nornalization schermes.

of 3. In the fits discussed above. we took $;=400 \mathrm{M} \cdot 1$ which is the value used by Kokoski and Isgur [5]. In addition to these fits. we performed sinmltaneons lits of both $q$ and 3 to the 28 decay widths see Figure 3.6 . The overall fits improved. but as usual some widths were in better agreement and some in worse agrerement with experiment when compared to the fits for,$t=100$ Mell. In gemeral we fonmel that the deviation in the results lies within the overall nucertainty wa assign to the tuodels. and so we choose to follow the literat ure and use , $5=400 \mathrm{M} \cdot \mathrm{L}$. 


\subsection{The $f_{4}(2220)$ Reexamined - What Is it Really?}

Th. $f_{4}(2220)$ (formerly known as he $\xi(2220)$ ) state was discovered by the Mark III Collaboration [16] in 1983, in the decay $e^{+} \epsilon^{-} \rightarrow J / \psi^{+} \rightarrow \gamma f_{4}(2220) \rightarrow \gamma \mathrm{K}^{++} \mathrm{K}^{-}-$ see Figure 3.7. It attracted considerable attention because of its narrow width of approximately $30 \mathrm{MeV}$, which was thought to be too small for a conventional meson of that mass. Over the years this has led to speculation that it might be a Higgs boson [17], a bound state of coloured scalars [18], a four quark state $[19,20]$. a $\Lambda \bar{\Lambda}$ bound state [21], or a meson hybrid or a glueball [22], among others (we only reference early works on each suggestion).

The $f_{4}(2220)$ has since been seen by other experiments, the results of which are summarized in Table 3.4. The rather broad state seen by DM2 is unlikely to be the same state seen by the other experiments. The recent measurements by the BES Collaboration are of particular interest, because the previously unseen $\pi^{+} \pi^{-}$and $p \bar{p}$ decay modes indicate a decay pattern that is roughly flavour-symmetric. in keeping with the glueball interpretation (see for example. the recent paper by (hao [23]). The $f_{4}(2220)$ has not been seen by $p \bar{p}$ experiments. but the limits set are not stringent enough to exclude the meson identification (for example see Barnes of al. [24]).

The intermediate state $J / \imath \cdot$ and the final observed states indicate that the parity and charge conjugation quantum numbers of the $f_{4}(2220)$ are ${ }^{++}$and that its spin $J$ must be even. although as can been seen from Table 3.4 the artual value of $J$ is rather uncertain. The Particle Data (iroup [1] has tentatively identified the $f_{4}(2220)$ as the ${ }^{3} F_{4}$ s.s meson (hence its presence in Table 1.2). but notes that this needs confirmation.

In order to determine whether the $f_{4}(2220)$ is a high-spin meson or something new and nuch more interesting. we need theoretical predictions of the properties of the various options in order to make the identification. In this work we carefully examine the decays of the ${ }^{3} F_{2}$ and ${ }^{3} F_{4}$ sis mesons in order to see if the meson identification is 


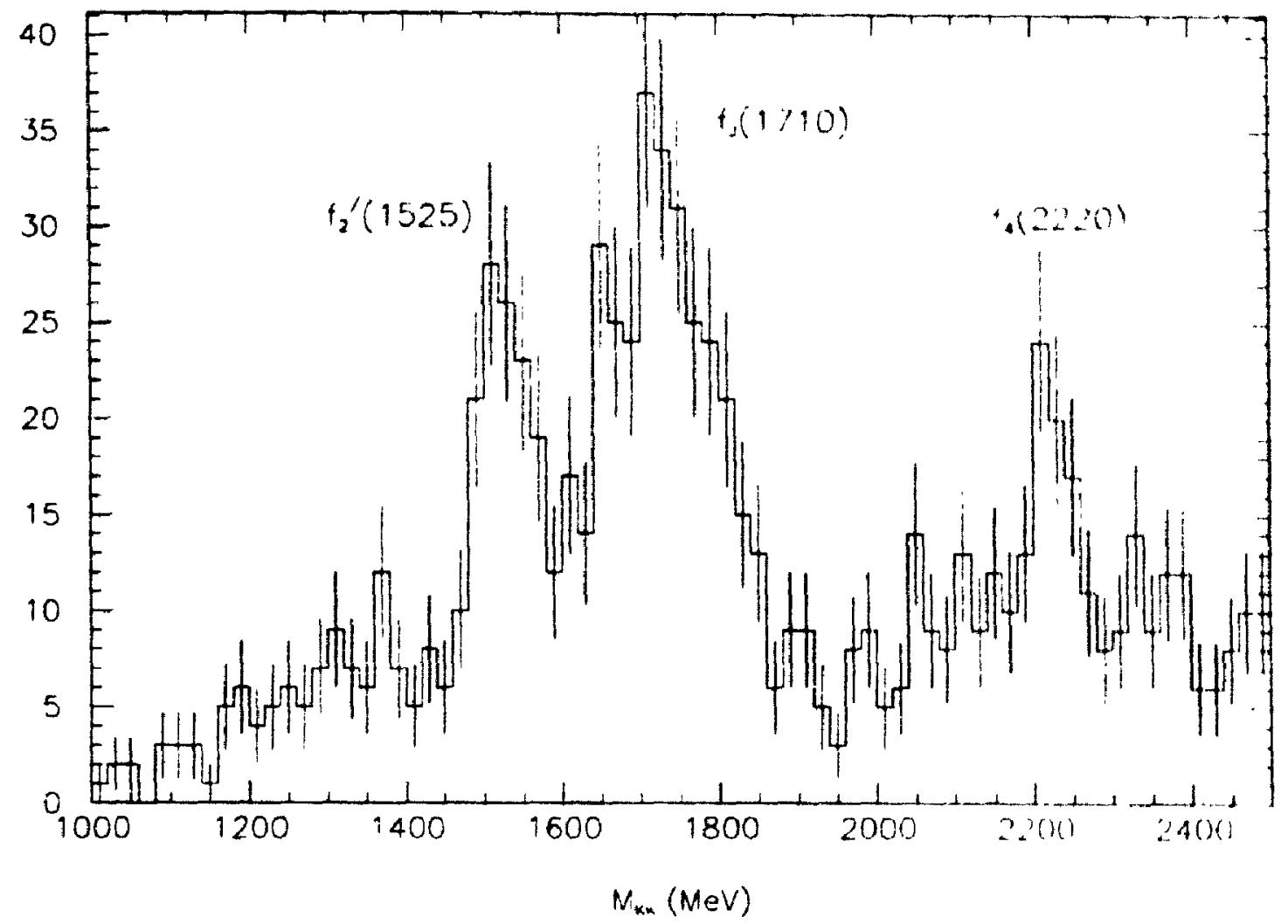

Figure 3.7: The original data from the discovery of the $f_{4}(2220)$ by the MARK III Collaboration [16]. The distribution of the $h^{+} \boldsymbol{K}^{-}$invariant mass in $e^{+} \rightarrow . / / e^{+} \rightarrow$ $\gamma \mathrm{K}^{+} \mathrm{K}^{-}$is shown, revealing three resonances. The errors show'n are purely stat istical.

tenable. These are the best meson candidates because of their $f^{\circ}$ s of $2^{++}$and $4^{++}$ respectively. and because their masses have been predicted to lie in the right range (Godfrey and Isgur [4] calculated masses of $2240 \mathrm{MeV}$ and $2200 \mathrm{MeV}$ respectively).

The decay widths of these mesons have been calculated previously. The first was by Godfrey, Kokoski and Isgur [30] using the flux-tube breaking model (and some.what different masses: $2270 \mathrm{MeV}$ and 2210 MeV respectively). Despite the fact that ome naively expects heavy mesons to be broad. since more pliase space and dechy chanmels lead to larger widths. they found the total widths to be appreximately go MeV and 50 MeV respectively. Based on production rate arguments. they concluded that the. $f_{4}\{2220)$ was most likely to be the ${ }^{3} F_{2}$ s.s state. However. Hor andyais was Hot 


\begin{tabular}{|c|c|c|c|c|}
\hline Experiment & Process & $\begin{array}{c}\text { Mass } \\
\text { (MeV) }\end{array}$ & $\begin{array}{l}\text { Width } \\
\text { (Mel) }\end{array}$ & $\mathrm{J}$ \\
\hline $\begin{array}{l}\text { SLA(:-SP-032 } \\
\text { (Mark III) }\end{array}$ & $\begin{aligned} \epsilon^{+} \epsilon^{-} \rightarrow J / \psi^{+} \rightarrow \gamma & f_{1}(2220) \\
& \rightarrow K^{+} h^{-} \\
& \hookrightarrow K_{S}^{0} \bar{h}_{S}^{00}\end{aligned}$ & $\begin{array}{l}2230 \pm 6 \pm 14 \\
2232 \pm 7 \pm 7\end{array}$ & $\begin{array}{l}26_{-16}^{+20} \pm 17 \\
18_{-15}^{+23} \pm 10\end{array}$ & \\
\hline $\begin{array}{l}\text { (GAMS-2000 } \\
\text { GAMS-4000) }^{b}\end{array}$ & $\begin{array}{c}\pi^{-} p \rightarrow \int_{4}(2220) n \\
\hookrightarrow \eta \eta^{\prime}\end{array}$ & $2220 \pm 10$ & & $\geq 2$ \\
\hline $\begin{array}{l}\text { SERP-E-147 } \\
\text { (MIS) }{ }^{\circ}\end{array}$ & $\begin{aligned} & \pi^{-} p \rightarrow f_{4}(2220) n \\
& \rightarrow K_{S}^{0} \tilde{h}_{S}^{0}\end{aligned}$ & $2230 \pm 20$ & $80 \pm 30$ & 2 \\
\hline $\begin{array}{l}\text { SLAC }- \text { E-135 } \\
\text { (LASS) }^{d}\end{array}$ & $\begin{aligned} & h^{-} p \rightarrow f_{4}(2220) \Lambda \\
& \rightarrow h^{2} h^{-}\end{aligned}$ & $2209_{-15}^{+17} \pm 10$ & $60_{-57}^{+107}$ & 4 \\
\hline RPP Average ${ }^{\circ}$ & & $2225 \pm 6$ & $38_{-13}^{+15}$ & $4 ?$ \\
\hline$\overline{(\mathrm{DM} 2)^{\mathrm{T}}}$ & $\begin{aligned} & c^{+}{ }^{-} \rightarrow J / \psi^{\prime} \rightarrow \gamma ? \\
& \hookrightarrow K_{S}^{0} \hat{K}_{S}^{0}\end{aligned}$ & $2197 \pm 17$ & $201 \pm 51$ & \\
\hline$(\mathrm{BES})^{\mathrm{s}}$ & $\begin{aligned} f^{+} \rightarrow J / \psi^{*} \rightarrow \gamma & f_{4}(2220) \\
& \hookrightarrow h^{+} h^{-} \\
& \hookrightarrow h_{s}^{0} \dot{K}_{S}^{0} \\
& \hookrightarrow p \bar{p} \\
& \hookrightarrow \pi^{+} \pi^{-}\end{aligned}$ & $\begin{array}{l}2230_{-7}^{+6} \pm 12 \\
2232_{-7}^{+8} \pm 15 \\
2235 \pm 4 \pm 5 \\
2235 \pm 4 \pm 6 \\
\end{array}$ & $\begin{array}{l}20_{-15}^{+20} \pm 12 \\
20_{-16}^{+25} \pm 10 \\
15_{-9}^{+12} \pm 9 \\
19_{-11}^{+13} \pm 12 \\
\end{array}$ & \\
\hline
\end{tabular}

Table 3.4: Summary of measurements of the $f_{4}(2220)$. The RPP average is for the first four entries in the table only: they feel that the $f_{4}(2220)$ still needs confirmation. In the case of the DM2 results. the resonance seen was not identified with the $f_{4}(2220)$. and so it is not included in the RPP average: the BES data is too recent.

- Reference [16].

D Refrerence [25].

- Reference [26].

" Reference [27].

- Reference [1].

Heference [28].

Reference [29].

cxhaustive in that it did not calculate the partial widths to all possible final states. In particular it made the assumption. which we will see to be incorrect. that the decays to an $i=J$ meson and a $h$ or $\eta$ were small on the basis of pluase space arguinents alone. ${ }^{10}$

\footnotetext{
"In addition. they note that the decay to $K^{*}(892) K^{*}(892)$ is dommated by the I. D partial wave
} 
In addition. Pakvasa. Suzuki and Tuan [20] have used Regge theory to estimate the partial width of the ${ }^{3} F_{4}$ ss. state decaying to two ${ }^{1} S_{0}$ mesons as approximately $40 \mathrm{Mel}$. with the total width expected to be a few times larger. And finall: Ono. Pène and Schöberl [31] have used the ${ }^{3} P_{0}$ model to calculate the partial widh of the ${ }^{3} F_{2} s \bar{s}$ state (assuming a mass of $2200 \mathrm{MeV}$ ) decaying to ${ }^{1} S_{0}$ and ${ }^{3} S_{1}$ states to be $417 \mathrm{MeV}$ (with the width to $h^{*}(892) h^{*}(892)$ dominant at $\left.34 \mathrm{~s}^{\circ} \mathrm{M}\right)$ ). In light of the new data, and these contradictory theoretical results, a new, complete calculation is required.

We have calculated the ${ }^{3} F_{2}$ and ${ }^{3} F_{4} s \bar{s}$ meson decay widths using the six combinations of model, wavefunction. and phase space/normalization discussed in Section 3.1. In order to judge the reliability of the results. it is useful to examine how well our calculations do for similar mesons. We do not have any good candidates for the ${ }^{2} \%_{2}$. but the $f_{4}(2050)$ and $\boldsymbol{K}_{4}^{*}(2045)$ differ only in flavour from the ${ }^{3} F_{4}$.s. Examining lighures $3.1,3.3$ and 3.5 , we see that for the five decays in question. the choice of phase. space/normalization makes a significant difference: RISSN tends to overestimate the partial widths, while KIPSN tends to underestimate them. For the has $(1780)$, which one would expect to behave similarly to the ${ }^{3} F_{4}$ states (since both are ${ }^{3} L_{l+1}$ ), the situation is unfortunately less clear. We can also examine the total widh of the $f_{4}(2050)$ and $K_{4}^{*}(2045)$ states. In Tables 3.5 and 3.6 we give the partial widths for all significant, kinematically-allowed decays and their sums, in the ${ }^{3} / 3$, morlel and with SHO wavefunctions only. It can be seen that once again the RPSN results are high, and the KIPSN results low. The maiti conclusisn we can draw from these results is that the widths of the ${ }^{3} F_{4}$ s. meson probably lie leet wern the RISN and KIPSS estimates but it is difficult to guess if they are cleser to the lower or upper value.

Turning now to our result for the ${ }^{3} F_{2}$ and ${ }^{3} F_{4}$ s.s mesons. in lables 3.7 and 3.5 we

(using the $S, L$ notation of Table 3.5) Unfortunatoly, $S=1$ is forbidden by conservallen of parily and charge conjugation. making the result for this dreay questiomals. 


\begin{tabular}{|c|c|c|c|}
\hline \multirow{3}{*}{$\begin{array}{c}\text { Decay } \\
f_{4}(2050) \rightarrow\end{array}$} & \multirow{3}{*}{$\begin{array}{c}\Gamma(\text { MeV) } \\
\text { from } \\
\text { Experiment }\end{array}$} & \multirow{2}{*}{\multicolumn{2}{|c|}{$\begin{array}{c}\text { (MeV) from Models } \\
{ }^{3} P_{0} \\
\text { SHO }\end{array}$}} \\
\hline & & & \\
\hline & & RPSN & KIPSN \\
\hline$[\pi \pi]_{0, G}$ & $35.4 \pm 3.8$ & 123 & 25 \\
\hline$[\pi \pi(1300)]_{0, G}$ & & 3.9 & 1.9 \\
\hline$\left[\pi a_{1}(1260)\right]_{1, f}$ & & 18 & 7.5 \\
\hline$\left[\pi a_{2}(1320)\right]_{2, F}$ & & 44 & 19 \\
\hline$\left[\pi \pi_{2}(1670)\right]_{2, l}$ & & 2.1 & 1.8 \\
\hline$[\rho \rho]_{0, G}$ & & 1.9 & 0.4 \\
\hline$[\rho \rho]_{2, D}$ & & 159 & 33 \\
\hline$[\rho \rho]_{2,(;}$ & & 7.3 & 1.5 \\
\hline$[\eta \eta]_{0 . c}$ & $0.4 \pm 0.2$ & 3.2 & 0.9 \\
\hline$\left[\eta \eta^{\prime}\right]_{0, G}$ & & 1.0 & 0.3 \\
\hline$\left[\eta f_{2}(1270)\right]_{2, F}$ & & 1.1 & 0.5 \\
\hline$\left.[\omega]_{2 . D}\right\}$ & $51+13 a$ & 50 & 10 \\
\hline$\left.[\omega \omega]_{2, r ;}\right\}$ & $34 \pm 13^{\circ}$ & 2.0 & 0.4 \\
\hline$[\boldsymbol{K} \bar{K}]_{\mathbf{Q}, G}$ & $1.4_{-0.4}^{+0.7}$ & 5.4 & 1.6 \\
\hline$\left[h \bar{h}^{\circ}(892)+c . c .\right\}_{1 . c}$ & & 2.7 & 0.8 \\
\hline$\left[\boldsymbol{K} \tilde{K}_{1}(1270)+c . c \cdot\right]_{1 . F}$ & & 2.3 & 1.2 \\
\hline$\left[h^{* *}(892) h^{*}(892)\right]_{2 . l}$ & & 7.3 & 2.1 \\
\hline Total $/ \sum \Gamma_{1}$ & $208 \pm 13$ & 435 & 109 \\
\hline
\end{tabular}

Table 3.5: The calculated partial decay widths and total width of the $f_{4}(2050)$ meson. The available experimental results [1] are also shown. The model results are given for two combinations of decay model. wavefunctions. and phase space/normalization (specified in the second. third and fourth rows of the heading. respectively). We have calculated the partial widths of all kinematically-allowed. OZI-allowed. twobody st rong decay's. but only show those partial widths that are $\geq 1 \mathrm{MeV}$ in at least one model. For this reason the total widths $n$, $r$ not equal the sum of the partial widths shown. The subscripts on the decays - $\therefore$ to the $S$ and $L$ (see Appendix E) of the given partial wave - the $L$ is in spectroscopic notation (S.P.D.F.G.H). We have used the neson mixings given in Appendix A.T where applicable.

"This number is the total for all partial waves.

give the partial widths for all significant. kinematically-allowed decays and their sums. for all six combinations of decay model, wavefunction. and phase space/normalization. For a degres of completeness, in Table 3.9 we also give the results for the other $L=3$. spintriplet ss meson. the ${ }^{3} F_{3}$. in the ${ }^{3} P_{0}$ model and with SHO wavefunctions only. 


\begin{tabular}{|c|c|c|c|}
\hline \multirow{3}{*}{$\begin{array}{c}\text { Decay } \\
h_{4}(2045) \rightarrow\end{array}$} & \multirow{3}{*}{$\begin{array}{l}\text { (Mel) } \\
\text { from } \\
\text { Experinent }\end{array}$} & \multirow{2}{*}{\multicolumn{2}{|c|}{$\begin{array}{c}\text { (Mel) from Models } \\
\text { SP' } \\
\text { SHo }\end{array}$}} \\
\hline & & & \\
\hline & & RPSN & kIISN \\
\hline$\overline{h \pi]_{0, G}}$ & $19.6 \pm 3.8$ & 5.5 & $1: 1$ \\
\hline$\left[\boldsymbol{K}^{\circ} \boldsymbol{\mu}\right]_{1, G}$ & & 19 & 1.1 \\
\hline$\left\{k^{\prime} b_{1}(123.5)\right\}_{1, F}$ & & 4.9 & 2.2 \\
\hline$\left[K a_{1}(1260)\right]_{1, F}$ & & 1.3 & 0.6 \\
\hline$\left[K a_{2}(1320)\right]_{2, F}$ & & 2.2 & 1.0 \\
\hline$\left[h^{\prime}(892) \pi\right]_{1, C ;}$ & & 23 & 5.5 \\
\hline$\left[h^{*}(892) \rho\right]_{2, D}$ & $18+10$ a & 76 & 18 \\
\hline$\left.\left[h^{*}(892) \rho\right]_{2, G}\right\}$ & & 2.1 & 0.5 \\
\hline$\left[K_{1}(1270) \pi\right]_{1, F}$ & & 1.6 & 0.7 \\
\hline$\left[h_{1}(1400) \pi\right]_{1 . F}$ & & 5.3 & 2.6 \\
\hline$\left[K_{2}(1430) \pi\right]_{2 . F}$ & & 5.2 & 2.6 \\
\hline$\left[\boldsymbol{L}^{\prime} \eta^{\prime}\right]_{\mathrm{c}, G}$ & & 3.3 & 0.9 \\
\hline$[\boldsymbol{h} \boldsymbol{w}]_{1, G}$ & & (i.0 & 1.1 \\
\hline$[\boldsymbol{h} \phi]_{1 . G}$ & & 1.1 & 0.4 \\
\hline$\left[K h_{1}(1170)\right]_{1 . F}$ & & 2.9 & 1.3 \\
\hline$\left[h f_{2}(1270)\right]_{2, F}$ & & 1.3 & 0.6 \\
\hline$\left[h^{*}(892) \eta\right]_{1,6}$ & & .1 .4 & 1.1 \\
\hline$\left[h^{*}(892)\right]_{2, D}$ & & 2.1 & 5.5 \\
\hline$\left[h^{\prime}=(892) \phi\right]_{2 . L}$ & $2.8 \pm 1 .-1$ & 3.2 & 1.0 \\
\hline Total $/ \sum_{2} \Gamma_{1}$ & $19 x \pm 30$ & 297 & (i.) \\
\hline
\end{tabular}

Table 3.6: The calculated partial decay widths and total width of the $\left.h_{i}(20) 15\right)$ mesom. The available experimental results [1] are also shown. For additional comments. sere Table 3.5.

a This number is actually for the final state $\left.h^{*}(8) 2\right) \pi \pi$. and in the total for all partial waves.

For the ${ }^{3} F_{2}$ ss state the main decay modes are. in desconding order. induding charge conjugate pairs:

$$
\begin{aligned}
& h_{1}(1270) h^{*} h_{1}(1270) h^{*}(x ! 2) . h^{*}(\times 92) h . h^{*}(\times 92) h^{*}(x+12) \\
& h_{2}^{*}(1430) h \cdot h \kappa \cdot f_{1}(1510) \eta
\end{aligned}
$$




\begin{tabular}{|c|c|c|c|c|c|c|}
\hline \multirow{4}{*}{$\begin{array}{l}\text { Decay } \\
3 F_{2} \text { s.s } \rightarrow\end{array}$} & \multicolumn{6}{|c|}{ T (MeV) from Models of Meson Decay } \\
\hline & \multirow{2}{*}{\multicolumn{2}{|c|}{$\frac{{ }^{3} P_{0}}{\mathrm{SHO}}$}} & \multicolumn{4}{|c|}{ Flux-tube Breaking } \\
\hline & & & \multicolumn{2}{|c|}{$\mathrm{SHO}$} & \multicolumn{2}{|c|}{$\overline{\mathrm{RQM}}$} \\
\hline & RPSN & KIPSN & RPSN & KIPSN & $\overline{R P S N}$ & KIPSN \\
\hline$h K] 0,1$ & 51 & 12 & $\overline{47}$ & 12 & 101 & $2: 3$ \\
\hline$\left[\boldsymbol{H}_{r} \boldsymbol{h}+\boldsymbol{c} \cdot \boldsymbol{c}\right]_{(\mathrm{n})}$ & 2.9 & 1.5 & 0.9 & 0.5 & 25 & 12 \\
\hline$H^{*}(h(92) h+c \cdot c)_{1, l}$ & 108 & 26 & 107 & 26 & 165 & 38 \\
\hline$\left[h^{\circ}(1410) h+c . c .\right]_{1, D}$ & 2.6 & 1.3 & 0.6 & 0.3 & 4.0 & 1.9 \\
\hline$\left.h_{1}(1270) h^{\circ}+c \cdot c\right]_{1 . l}$ & 445 & 187 & 449 & 194 & $10 \div 2$ & 426 \\
\hline$\left[\mu_{1}(12 \gamma(0) h+c . c]_{1, f}\right.$ & 25 & 11 & 27 & 12 & 41 & 16 \\
\hline$\left[h_{1}(1.100) h^{\circ}+\right.$ c.c.l. & 14 & 6.3 & 15 & 6.9 & 29 & 12 \\
\hline$\left[K_{1}(1400) h+\text { c.c. }\right]_{1 . F}$ & 0.8 & 0.1 & 1.0 & 0.1 & $\sim 0$ & $\sim 0$ \\
\hline$\left[K_{2}(1.430) h+\text { c.C. }\right]_{2 . P}$ & 5.4 & 24 & $5 \pi$ & 25 & 112 & 17 \\
\hline$[K(1430) h+c . c .]_{2 . t}$ & 9.6 & 1.3 & 10 & 4.7 & 22 & 9.1 \\
\hline$\left[h^{\circ}(8 \div 2) h^{*}(892)\right]_{0 . D}$ & 24 & 5.7 & 21 & 5.9 & 39 & 8.9 \\
\hline$\left[h^{*}(892) h^{-}(892)\right\}_{2.1}$ & 11 & 3.3 & 11 & 3.4 & 23 & 5.1 \\
\hline$\left[h^{\circ}(892) h^{*}(892)\right]_{2 . K}$ & 48 & 12 & 52 & 13 & 83 & 19 \\
\hline$\left[h_{1}(1270) h^{*}(892)+c .\right]_{1 . P}$ & 99 & 40 & 102 & 42 & $20 \%$ & 79 \\
\hline$\left[h_{1}(1270) \dot{h}^{*}(892)+c . c\right]_{1 . F}$ & 0.5 & 0.2 & 0.6 & 0.2 & 1.1 & 0.4 \\
\hline$\left[h_{1}(12 \pi 0) h^{*}(892)+\text { c.c. }\right]_{2, P}$ & $3: 3$ & 13 & 34 & 14 & 70 & 26 \\
\hline$\left[K_{1}(1270) K^{*}(892)+c . c .\right]_{2 . F}$ & 0.8 & 0.3 & 0.9 & 0.4 & 1.8 & 0.7 \\
\hline$[\eta \eta]_{0 . D}$ & 14 & 3.3 & 13 & 3.2 & 20 & 4.1 \\
\hline$\left[y^{\prime} \eta\right]_{0 . D}$ & 29 & 7.0 & 29 & 7.2 & 29 & 6.6 \\
\hline$\left[f_{1}(1510) \eta\right]_{1, P}$ & 45 & $2 \cdot 2$ & 46 & 24 & 92 & $4: 3$ \\
\hline$\left[f_{2}^{\prime}(1525) \eta\right]_{2 . P}$ & 14 & 6.9 & 11 & 7.3 & 29 & 11 \\
\hline$\left[\eta^{\prime} \eta^{\prime}\right]_{0, D}$ & 6.6 & 1.6 & 6.7 & $1 . i$ & 4.9 & 1.1 \\
\hline$[\phi \phi]_{0 . D}$ & 3.9 & 1.2 & 3.4 & 1.3 & 5.5 & 1.6 \\
\hline$[o g]_{2, D}$ & 2.2 & 0.7 & 2.3 & 117 & 3.1 & 0.9 \\
\hline$[\phi \phi]_{2, i}$ & 1.0 & 0.3 & 1.0 & 0.3 & 1.1 & 0.3 \\
\hline$\sum_{1} I_{1}$ & 10.46 & 391 & 1058 & 406 & 2181 & 797 \\
\hline
\end{tabular}

Table 3.7: The calculated partial decay widths and total width of the ${ }^{3} F_{2}$ s.s meson. We do not include a decay to $f_{0}(980) f_{0}(980)$ because we question its assignment as a " $I_{0}$ meson. At a more likely mass for the ${ }^{3} P_{0}$ ss meson. this decay is kinematically inaccessible. For additional comments. see lable 3.5 .

a ${ }^{*}$ is our notation for the first radial excitation $\left(2^{\prime} S_{0}\right)$ of the $h$. 


\begin{tabular}{|c|c|c|c|c|c|c|}
\hline \multirow{4}{*}{$\begin{array}{l}\text { Decay } \\
{ }^{3} F_{4} \text { s.s } \rightarrow\end{array}$} & \multicolumn{6}{|c|}{ T (Mol) from Models of Meson Deeay } \\
\hline & \multirow{2}{*}{\multicolumn{2}{|c|}{$\frac{3 P_{0}}{\mathrm{SHO}}$}} & \multicolumn{4}{|c|}{ Fux-tulu* Broaking } \\
\hline & & & \multicolumn{2}{|c|}{ SHO } & \multicolumn{2}{|c|}{$\mathrm{KQM}$} \\
\hline & KPSN & KIPSN & RPSN & KIPSN & RIISN & KIIST \\
\hline$h h_{\text {U.G }}$ & 118 & 29 & 105 & 31 & (i!) & 1.1 \\
\hline 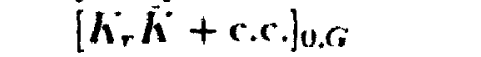 & 0.7 & 0.4 & 0.1 & (1).2 & 2.1 & 1.2 \\
\hline$\left[K^{\circ}(892) K^{\circ}+\text { c.c. }\right]_{1.6}$ & 107 & 27 & $11:$ & 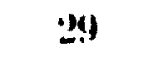 & $11: 3$ & 26 \\
\hline$\left[h^{*}(1410) h+c . c .\right]_{1 . C i}$ & 1.7 & 0.9 & 0.8 & 0.1 & 5.0 & 2.1 \\
\hline$\left[h_{1}(1270) h+c . c\right]_{1, F}$ & 6.4 & 2.8 & 7.0 & 3.1 & 10 & 1.1 \\
\hline$\left[k_{1}(1270) h+c . c .\left.\right|_{1 . H}\right.$ & 1.3 & 0.6 & 1.4 & 0.6 & 3.7 & 1.5 \\
\hline$\left[K_{1}(1400) \tilde{K}+\text { c.c. }\right]_{1, F}$ & 1.4 & 6.1 & 15 & i.11 & $2 !$ & 12 \\
\hline$\left[h_{2}(1430) h+c . c \cdot\right]_{2 . r}$ & 15 & 7.0 & 16 & $T .7$ & 35 & $1: 1$ \\
\hline$\left[h^{\prime}=(892) h=(892)\right]_{0, G}$ & 2.1 & 0.5 & 2.3 & 0.6 & 4.3 & 1.11 \\
\hline$\left[K^{\prime}(892) K^{-}(892)\right]_{2 . D}$ & 181 & 14 & 18.1 & $.16 i$ & $31:$ & 71 \\
\hline$\left[K^{*}(892) h^{*}(892)\right]_{2 . G}$ & 8.2 & 2.0 & $8 . !$ & 2.2 & 17 & $: 3 . !$ \\
\hline$[\eta \eta]_{0 . G}$ & 11 & 3.5 & 15 & .3 .9 & 5.11 & 1.2 \\
\hline$\left[\eta^{\prime} \eta\right]_{0.6}$ & 6.4 & 1.7 & $7 . \pi$ & 1.4 & 2.1 & 11.6 \\
\hline$|\infty O|_{2 . D}$ & 20 & 6.6 & 21 & 7.1 & $: 31$ & 9.5 \\
\hline$\sum_{1} \Gamma_{1}$ & 495 & $13: 2$ & $5: 2$ & 1.12 & fii3:1 & Ilifi \\
\hline
\end{tabular}

Table 3.8: The calculated partial decay nidths and total width of the $1 \%$, whesoll. For additional comments, sere Tables 3.5 and 3.7 .

It is clearly a mistake to neglect the decays fo all $I .=1$ meesoul allal a $\mathrm{h}$ of $\mathrm{g}$ : ly! far the largest partial width is for the decay to $K_{1}(1270) h_{\text {. and milly of }}$ the ot her decays involving $L=1$ mesons are significant. The results Isiug RQMI wavefunctions are surprisingly large about double those using SHO wavefunctions. Turning to the results with SHO wavefunctions. we see that if we believe that the KII'SX renult give a lower bound for the ${ }^{3} F_{2}$ as well as the ${ }^{3} F_{1}$. we call expert a total width $\sim$ foll Mel. in keeping with naive expectations. Even if this width ia ton large loy at factor of 2.

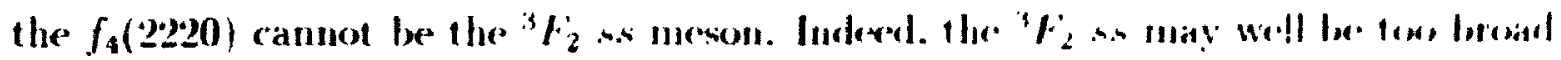
to be found experiment ally.

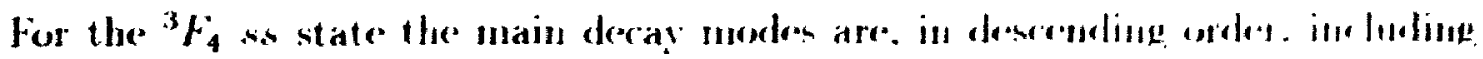




\begin{tabular}{|c|c|c|}
\hline \multirow{3}{*}{$\begin{array}{l}\text { Decay } \\
F_{3} \text { ss } \rightarrow\end{array}$} & \multirow{2}{*}{\multicolumn{2}{|c|}{$\begin{array}{c}\Gamma(\text { MeV }) \text { from Models } \\
{ }^{3} P_{0} \\
S H O\end{array}$}} \\
\hline & & \\
\hline & PPST & KIPST \\
\hline$\overline{\left(h^{\circ}(892) \hat{K}+c . c \cdot\right]_{1 . l}}$ & $\overline{154}$ & 38 \\
\hline$\left[h^{\bullet}(892) h+c . c .\right]_{1, r}$ & 98 & 24 \\
\hline$\left[K^{-}(1410) h+c . c \cdot\right]_{1, b}$ & 2.5 & 1.3 \\
\hline$\left[K^{*}(1410) h+c c \cdot\right]_{1, c}$ & 2.2 & $1 . !$ \\
\hline$\left[h_{0}^{*}(1430) h+c \cdot c\right]_{0 . F}$ & 5.3 & 2.4 \\
\hline$\left[K_{1}(1270) K+c . c\right]_{1, F}$ & 1 & 30 \\
\hline$\left[K_{2}^{*}(1430) K+c \cdot\right]_{2,}$, & 30:5 & $13 \pi$ \\
\hline$\left[h^{\bullet}(892) h^{\bullet}(892)\right]_{2, l}$ & 66 & 16 \\
\hline$\left[h^{\circ}(892) h^{\circ}(892)\right]_{2, c i}$ & $2 \pi$ & 6.6 \\
\hline$\left[h_{1}(1270) h^{*}(892)+c \cdot c\right]_{2, P}$ & 157 & 63 \\
\hline$\left[f_{2}^{\prime}(1525) \eta\right]_{2, P}$ & 73 & 37 \\
\hline$[\phi \phi]_{2, \nu}$ & 10 & 3.2 \\
\hline$\sum_{1} i_{i}$ & 9.4 & 360 \\
\hline
\end{tabular}

Table 3.9: The calculated partial decay widths and total widt h of the "${ }^{3} F_{3}$ s.s meson. We do not include derays to $f_{0}(9 \times 0) \eta$ or $f_{0}(980)$, because we quest ion the assignment of the $f_{0}(980)$ as a ${ }^{3} P_{0}$ meson. At a more likely mass for the ${ }^{3} P_{0}$ ss meson. this decay is kimematically inaccessible. For additional comments. see Table 3.5.

charge conjugate pairs:

$$
h^{*}(892) h^{*}(892) . K K . h^{*}(892) h .00
$$

Note that the total width of the ${ }^{3} F_{3}, s$ is quite a bit less than that of the ${ }^{\circ} F_{2}$ s.s. This can be understood in terms of the orbital angular moment um between the mesons in the tinal state. Because of the higher spin of the ${ }^{3} I_{4}$. it tends to rield find states with higher orbita! angular moment um in order to conserve angular moment um. These decayss are then suppressed by the higher angular moment um barrier. Examining Table 3.7. we see that the lowest orbital angular moment um final states in ${ }^{3} F_{2}$... deray are in P'waven. All of these decay's are relatively broad bat the decay to $k_{1}(1270) h$ is the P-wave decay with the largest available phase space. In fact. one 
could almost order the P-wave derays using phase space aloute. The analenguss deray of the ${ }^{3} F_{4}$ ss is in an $F$-wave and therefore is suppressed by a larger angular momentum

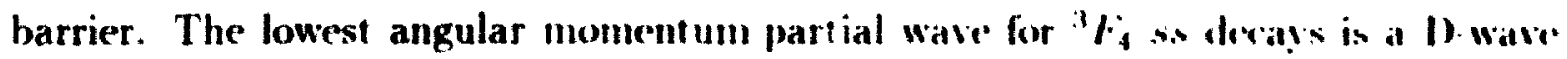
which. although it has the largest partial width of all $1 \%$... decays. is still smalley than the P-wave ${ }^{3} F_{2}$ s.s decay:

Looking at the total width of the "F, state. if we accept that the true renth lies between those obtained from RPSX and KIPSX. then we would expert the wilth to be $\gtrsim 1.40 \mathrm{Mel}$ and $\leqslant 600 \mathrm{Mel}$. While this in not rery speritic. We are really interested in the lower bound, and whether this could be the farentil. It is paxilhe.

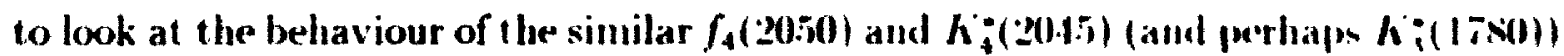
decays in selected model, wavefunction and phase spactefuormalization combinations. For example. in the ${ }^{3} P_{0}$ model with SHO wavefunetions and IRI'SI. the deray: to two ${ }^{3} s_{1}$ mesons are well predicted. indicating that the largest partial width of the " s. s. (to $\left.h^{*}(892) h^{*}(892)\right)$ may well be close to the calculated valine. (On the other hand. the partial widths to two 's. mesons may be less than predicted, and to a 'satud at

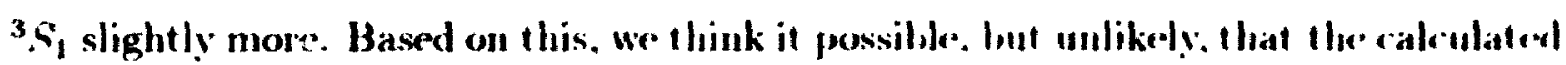

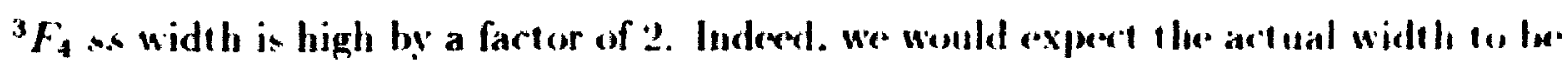

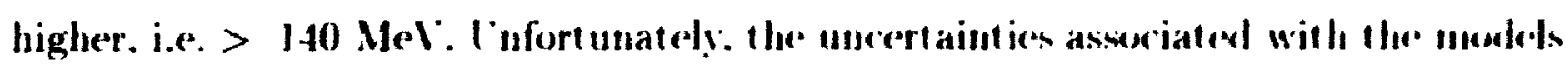

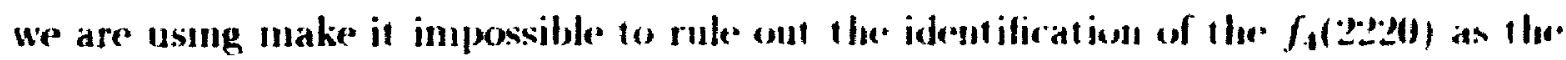
${ }^{3} F_{4}$ s. meson with any surety: howerer. ne foel it is mulikely:

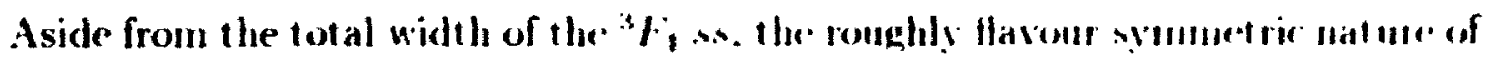
the $f_{4}(2220)$ decays observed at BES suggent that it is mot an ... mesoun, sind e prumlue tion of $\pi \pi$ and $p$ would be (OZ1-suppressed. Wie thes propeme a second explanation of what is being observed in this mase region that two different hadron statem are

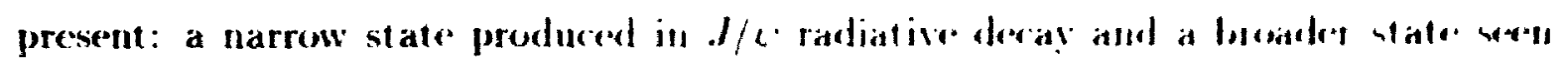

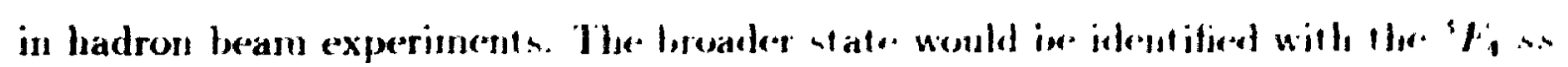

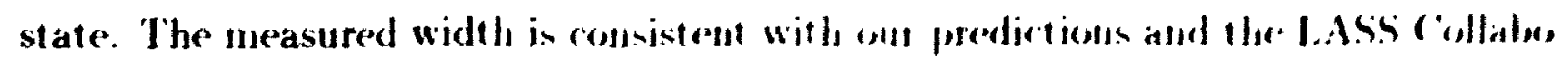


ration [27] shows evidence that its quant um nunbers are $f^{m}=1^{++}$. We would then identify the narrow hadron stato observed in the glnon rich $J / c$ radiative decals as a glueball [2:3]. The narrow state is not sere in hadron beam production coause it is narrow. is produced weakly in these experinents through intermediate gluons. and is hiddery by the s.s state. (onversely. the broader state is not seen in $\mathrm{l} / 2$ radiative derays berause this mode preferentially produces states with a high glue content. Crucial lo this explanation is $t^{\circ}$ experimental veritication of the BES results on the Havour symmetric couplings of the state pros ed in J/e radiative decal and the

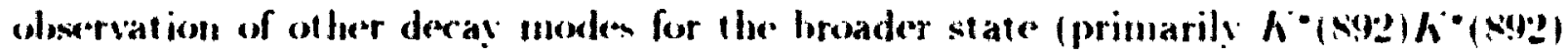
and $\left.h^{*}(\mathrm{~s}: 2) h^{\circ}\right)$ in addition to the theoretical verification that the predicted tensor ghemall is as harrow as the observed nidtli.

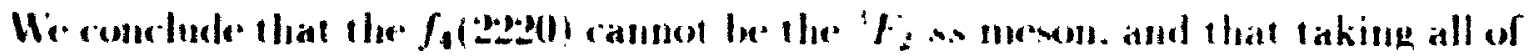

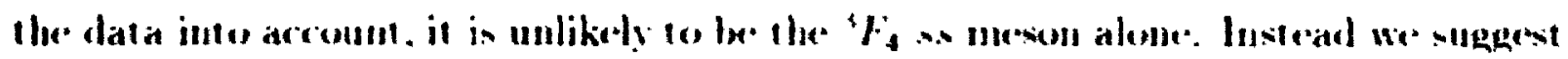

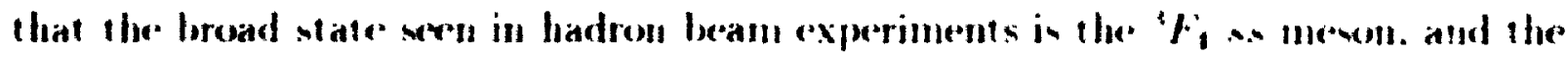

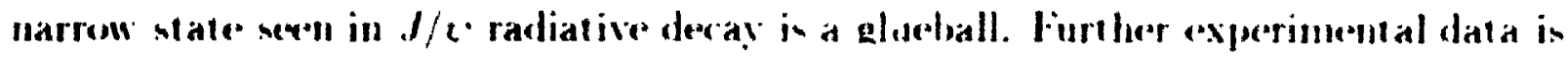
novedeal to tinally identify the $\left.f_{4}(2)=20\right)$.

\subsection{Constraining the $K_{i}$ Mixing Angle}

As discussed in Appendix A.7. the ' $P_{1}$ and ' $I$ ' thange menche mix lo produce the

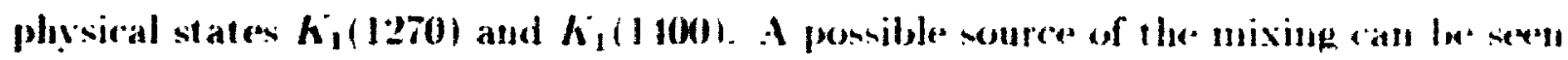
in the spin-orbit portion of the Ilamiltonian of (iedfrey and lsqur - relativized quark

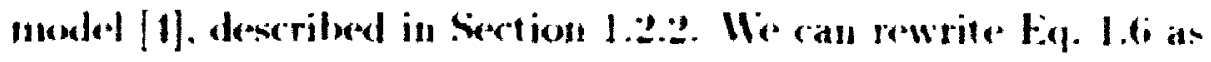

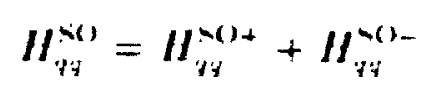


where

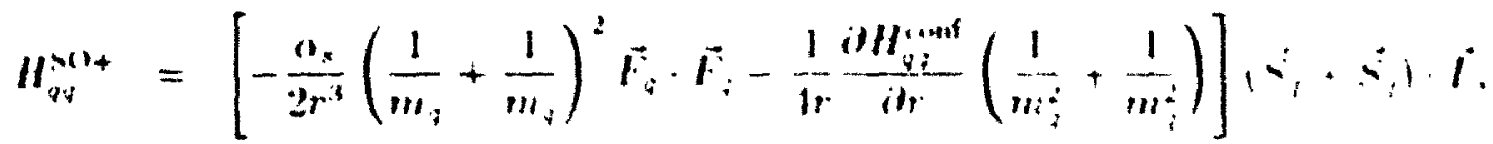

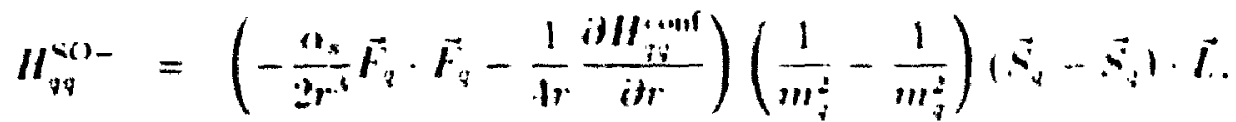

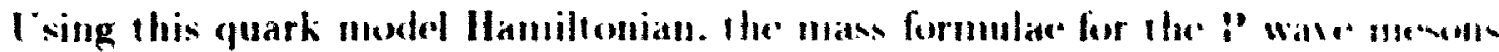
are

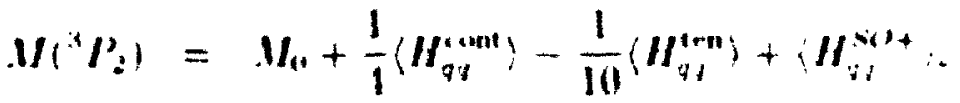

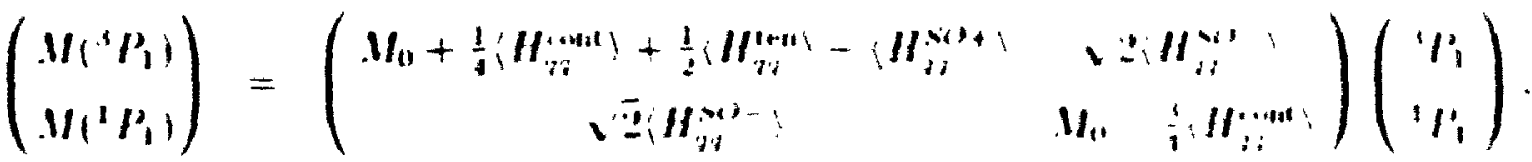

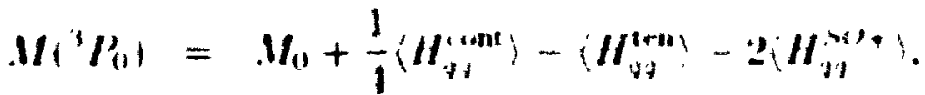

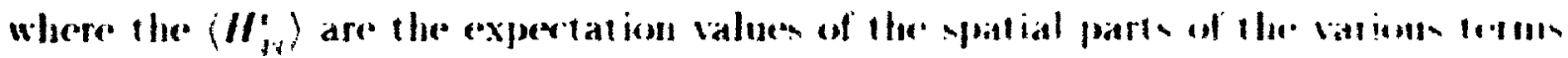

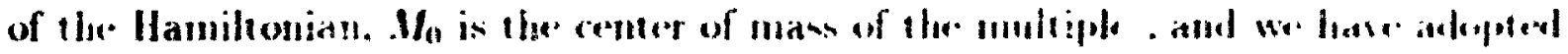

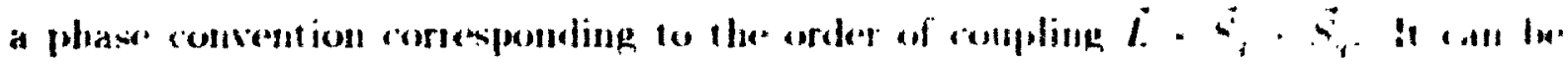

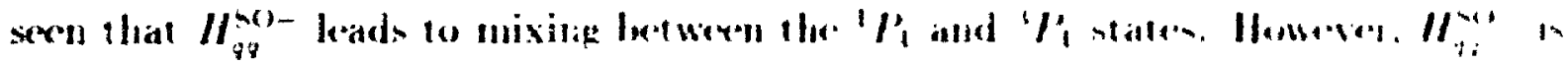

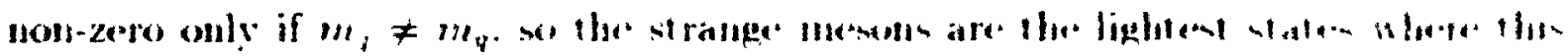
mixing can occous.

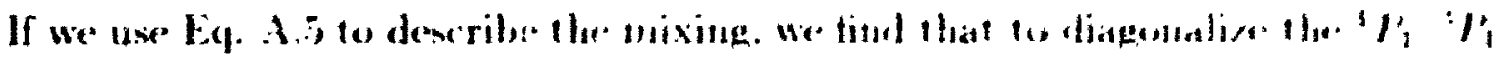
Hamiltomian we must hate.

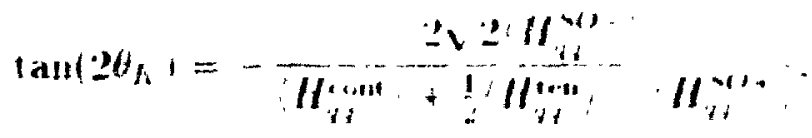

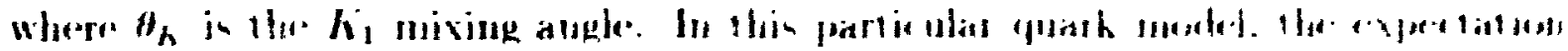

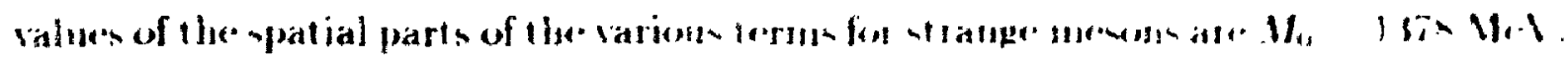

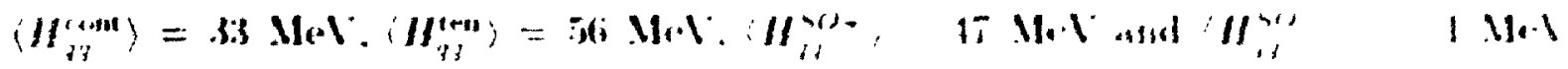


[32]. This gives rise to a prediction of $\theta_{k}=6^{\circ}$.

In this section we compare the predictions of the ${ }^{3} P_{0}$ and flux-tube breaking inociels of mesoil decav to experimental data for five decay widths and two ratios of 1) to $S$ amplitudes $"$, in order to determine $\theta_{k}$. This mixing angle is of interest both for its own sake, and for what it can can tell us about the quark model Hamiltonian.

The $K_{1}(1270)$ and $K_{1}(1400)$ can both decay to $K^{\prime} \rho$ and $K^{*}(892) \pi$; the $h_{1}(1400)$ can also deray to $f w$. All of the decays occur in the partial waves $1, S$ and $1, D$ (in $S, L$ notation $\rangle$. Alt hough decays to other final states are observed they lie below threshold and proceed through the tails of the Breit-Wigner resonances making the calculations less reliable. In addition to these five decay widths, there are also experimental results available for the ratio of $\mathrm{D}$ to $S$ amplitudes for both $h_{1}(1270)$ and $h_{1}(1400)$ decaying to $h^{* *}(892) \pi$.

We have calculated these seven quantities using the six combinations of model. wavefunction, and phase space/normalization selected in Section 3.1. The $K_{1}(! 270)$ quantities are plotted as a function of the $h$, mixing angle in Figure 3.8. and the $h_{1}(1400)$ quantities are plotted in Figure 3.9 - the experimental results are also shown in both figures.

In order to determine the best fit values of $\theta_{k}$. we have carried out a least squares fit for cach of the six combinations of decay model. wavefunction. and phase space/normalization. The best fit values of $\theta_{k}$ and their corresponding $1^{2} /$ dof are shown in Table 3.10. as are the resulting values of each of the seven quantities being considered. and their experimental values. Examining the results. we see that as usual. the values of $1^{2} /$ dof are high. but not as high as were obtained in the 28 -decay fits of section 3.1. This is not unexpected. since the similar decay $b_{1}(1235) \rightarrow \omega \pi$ was described reasonably well in those fits. It is also clear that the $\$ H O$ wavefunctions

\footnotetext{
"is "ratio of 17 to $S$ amplitudes" we mean the rato of the $L=2$ partial wave amplitude for a (i. $\because$ to the $L=0$ partial wave amplitude for the same decay.
} 


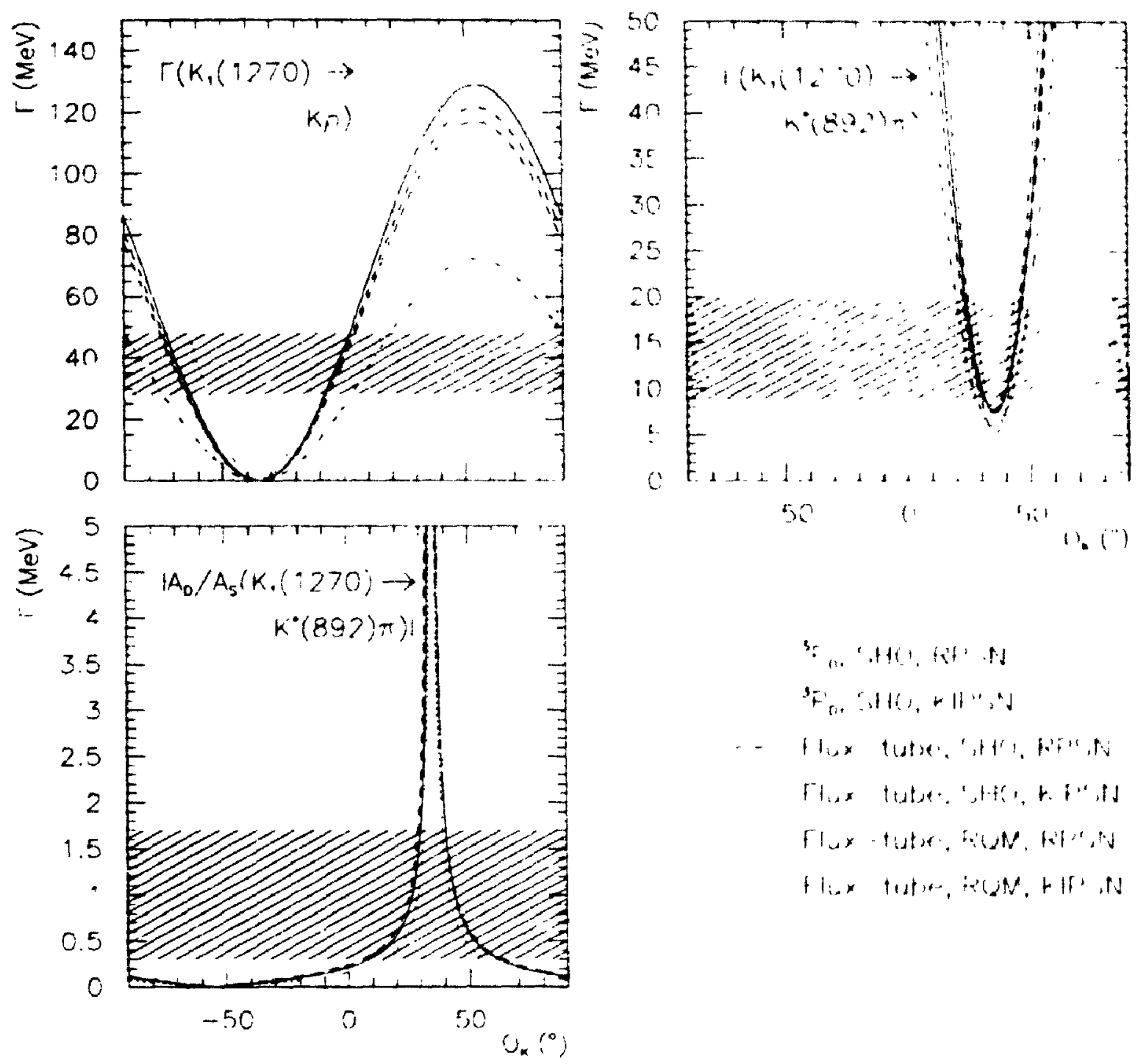

Figure 3.8: Graphs of the $h_{1}(1270)$ partial decay widths ald ratio of I) to 4 all plitudes considered in this work, vs. the mixing angle $\theta_{h}$. The sharded regioms

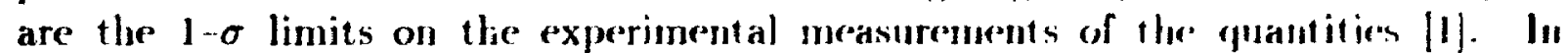
the key the labels refer to the meson decay model, the wavefunctione moded. and the type of phase space/normalization used. Only three curves arre show. for $\left|A_{D} / A_{s}\left(h_{1}(1270) \rightarrow h^{*}(892) \pi\right)\right|$. because the differences between flesi and lills: cancel in the ratio of amplitudes. making the RPSS and KIISS curves for the sime model and wavefunctions jdentical.

do better than the RQM. and in terms of phase spare/mormalizaliun. Fillesid does better than the RPSN. 


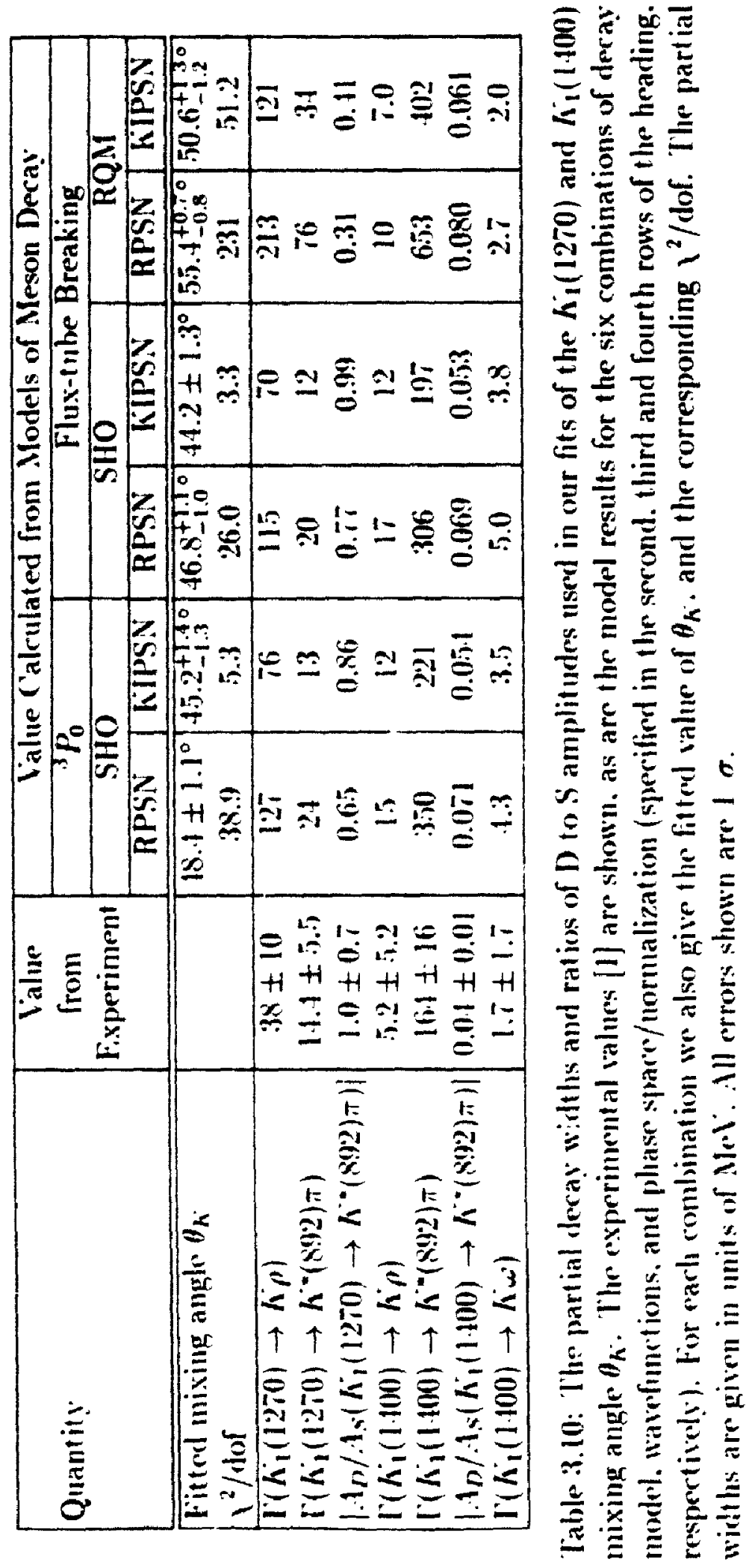



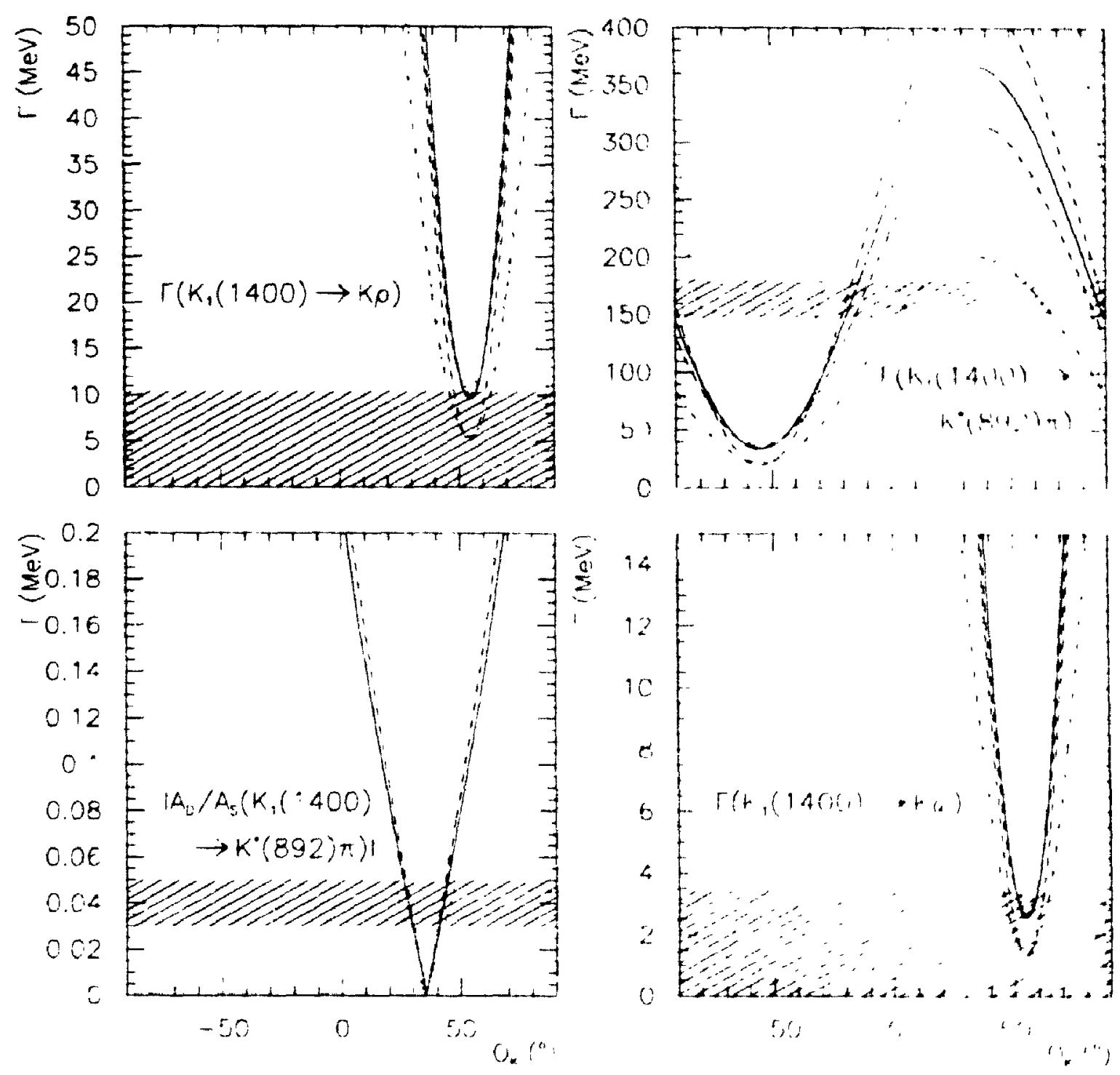

Figure 3.9: Graphs of the $h_{1}(1400)$ partial decay widths and ration of 11 to 5 atul

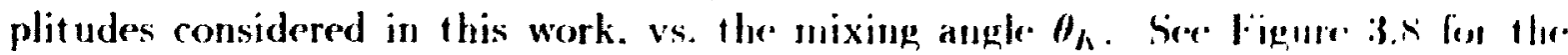
explanation of the line labels and additional netes.

The resulting values of $\theta_{k}$ are all fairly similar, falling in the range 11 iffin The lowest values of $1^{2} /$ dof oreur for fits near $11^{6}$. and se we take that in coll approximate result. What can this tell us aleont the quatk model Hamiltomian?" A

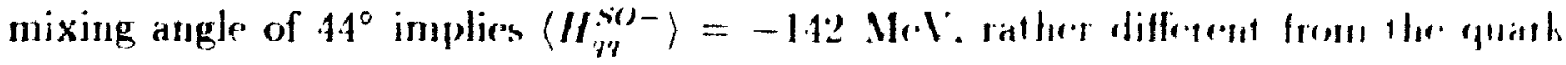

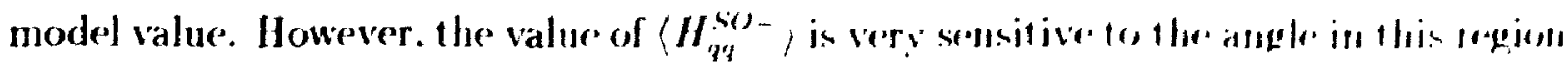


(r.g. $\theta_{K}=11^{\circ}$ implies $\left\langle H_{q q}^{S O-}\right\rangle=-3.5 \mathrm{MeV}$, while $\theta_{K^{\circ}}=45^{\circ}$ implies $\left\langle H_{q q}^{S O-}\right\rangle=-x$ ). so the only conclusion we can really draw is that the quark model value is very low. if $\left\langle\mathrm{H}_{\mathrm{qq}}^{\mathrm{SO}}\right\rangle$ is responsible for all of the mixing. We cannot rule out the possibility that some other interaction is partly responsible for the mixing, such as mixing via common decay channels.

To consider the SO Hamiltonian further, define $\bar{m}=\frac{1}{2}\left(m_{q}+m_{q}\right)$ and $\Delta=$ $\left(m_{q}-m_{q}\right)$. Next expand $1 / m_{q}=1 / m_{1}+\Delta /\left(2 \bar{m}^{2}\right)+\ldots$ and $1 / m_{\bar{q}}=1 / \bar{m}-\Delta /\left(2 m^{2}\right)+\ldots$ to first order in $\Delta / m$ (each of these truncated results has less than an $11 \%$ error for the quark masses we used). In terms of these. the contributions to the spin-orbit Ilamiltonian berome

$$
\begin{aligned}
& H_{q q}^{S O+} \simeq\left(-\frac{2 a_{s}}{m^{2} r^{3}} \vec{F}_{q} \cdot \vec{F}_{q}-\frac{1}{2 m^{2} r} \frac{\partial H_{q q}^{\text {conf }}}{\partial r}\right)\left(\vec{S}_{q}+\vec{S}_{q}\right) \cdot \vec{l} . \\
& H_{q q}^{S Q+} \simeq \frac{\Delta}{m}\left(-\frac{a_{s}}{m^{2} r^{3}} \vec{F}_{q} \cdot \vec{F}_{q}-\frac{1}{2 m^{2} r} \frac{\partial H_{q q}^{\text {conf }}}{\partial r}\right)\left(\vec{S}_{q}-\vec{F}_{q}\right) \cdot \vec{l} .
\end{aligned}
$$

The $\left\langle\left(\vec{S}_{q} \pm \vec{S}_{q}\right) \cdot \vec{L}\right\rangle$ factors are roughly the same size. and the $\Delta / \vec{m}$ term is not toc) far from 1 (it is $\geq \frac{1}{2}$ for the quark masses we used). so the factors in the large parembeses cont ribute at the same oucer of magnitude. Note that the contributions from the Thomas precession term are equal. but those from the colour magnetic term differ by a factor of 2 . The model gives a large $\left\langle H_{q \bar{q}}^{\mathrm{SO}+}\right\rangle$ of $47 \mathrm{Me}^{2}$. but a small $\left\langle H_{q q}^{\mathrm{s}()_{-}}\right\rangle$of -1 Mel arising from a delicate rancellation of the colour magnetic and Thomas precession terms. It is thus concejable that a small change in the model could lead to a substantially larger $\left\langle H_{q \bar{q}}^{\text {so- }}\right\rangle$. so we are unable to draw any conclusions about whether the mixing is largely due to another mechanism. or the quark model Hamiltonian just meeds some retuning. 


\section{Chapter 4}

\section{Effects of Final State Interactions}

\subsection{Introduction to Final State Interactions}

Final state interactions (FSI's) refer to additional interactions betwern part irles in the final state that are not included in the basic interaction.' For example, in ("hiptier ts. we consider the basic interaction $\eta \rightarrow \pi^{+} \pi^{-}$, which proceds through a scalar Q(1:J) vertex. However. there will also be a $Q(1)$ interaction between the linal slate pions. In Chapter 5. we attempt to calcuiate the effects of the FSI for this process In this chapter. we int roduce the techniques that we will apply to the problem of F:SI: : [:3:3].

The techniques involve nonrelat ivist ic quant un mechanies (including the Sirhrö dinger equation), corrected to include relativistic pluase space where possible. The. basic interaction may still be calculated relativistically: a.g. iो $\rightarrow \pi \pi$. This mixture. of nonrelativistic and relativistic element n is typical of the quark model.

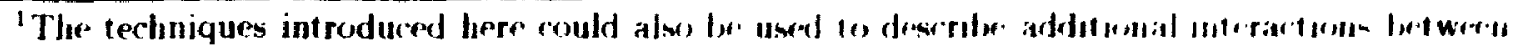

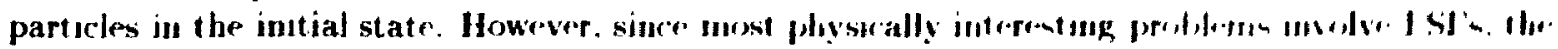

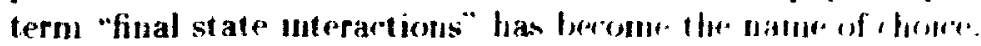




\subsection{Solving the Schrödinger Equation with the FSI Potential}

Our first step in calculating the effects of an FSI is to find the wavefunction describing the state of the final state particles due to the presence of the FSI only. We assume that the FSI can be described by a potential between the final state particles. and that the wavefunction can be found by solving the Schrodinger equation.

Th" relative wavefunction for two bodies in a potential that only depends on their relative position $\vec{r} \equiv \vec{r}_{1}-\vec{r}_{2}$ can be found from the Schrödinger equation for a central-force potential

$$
\left[\Gamma_{r}^{2}-2 \mu I(r)+k^{2}\right] \cdot \cdot(\vec{r})=0
$$

where $\mu$ is the reduced mass of the particles. $I(r)$ is the potential bet ween the two farticles and $h$ is the relative momentum between the particles in the absence of a potential. given ly $k^{2} \equiv 2 \mu E$. where $E$ is the kinetic energy of the system. Recall that wo are using the particle physics convention that $c \equiv \hbar \equiv 1$.

If we now select a solution with a particular orbital angular moment um and set $v^{\prime \prime m}(\vec{r}) \equiv R_{l}(r) V_{i m}\left(\Omega_{r}\right)$, then $R_{l}(r)$ satisfies the radial equation

$$
\left[\frac{1}{r} \frac{\mathrm{d}^{2}}{\mathrm{~d} r^{2}} r-\frac{l(l+1)}{r^{2}}-2 \mu l(r)+k^{2}\right] K_{l}(r)=0
$$

If we further set $K_{l}(r) \equiv y_{!}(r) / r$. then $y_{l}(r)$ satisfies the equivalent radial equation

$$
\left[\frac{\mathrm{d}^{2}}{\mathrm{~d} r^{2}}-\frac{l(l+1)}{r^{2}}-2 \mu l(r)+k^{2}\right] y_{l}(r)=0 \text {. }
$$

The solution of Eq. 1.1 for $I(r)=0$ is just a plane wave. which an be broken 
up into its partial waves.

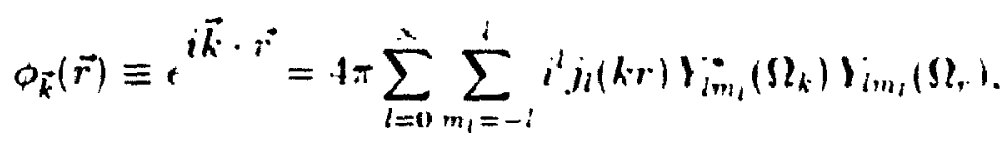

where $j_{l}(k r)$ is a spherical Bessel function and $\vec{k}$ gives the direction of the incident particle. The equivalent solution of Eq. 4.2 for $l(r)=0$ (picking the only particular solution that is regular at the origin) is

$$
R_{l}(r)=j(k \cdot r) \stackrel{k r \rightarrow g}{\rightarrow} \frac{1}{k r} \sin \left(k \cdot r-\frac{1}{2} i \pi\right)
$$

For the case with a potential. the stationary seatlering wales $\ell_{i}^{ \pm}(r)$ are solutions of F. 4.1. Their asymptotic forms consist of the incoming plane wave. and either all

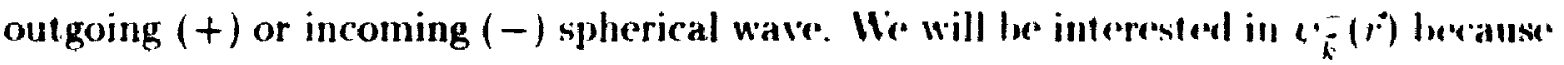
it will be describing the final state. and hence must be propagated backwards in tinne as an incoming spherical wave to be projected onto the initial statc platre waves at an equal time. In nonrelativistic collision theory [34] it is defined lo have the follewing asymptotic form

$$
\vec{r}(\vec{r}) \stackrel{\rightarrow \rightarrow}{\rightarrow}, \vec{k} \cdot \vec{r}+f_{\vec{i}}(\Omega,) \frac{-1 k r}{r}
$$

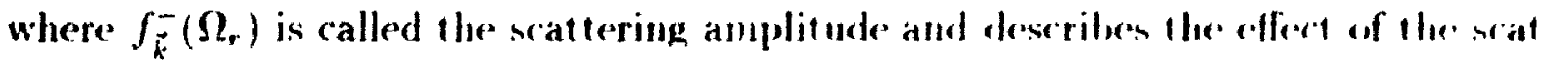
tering.

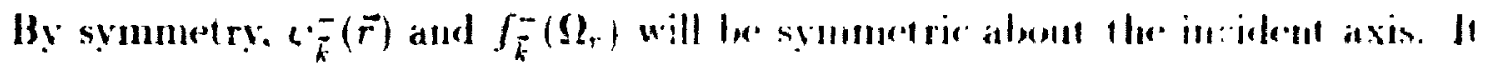

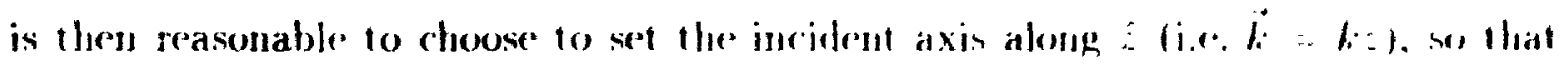
neither function is dependent on $o_{r}$. He an then make the following expandeme in terms of Legendre polynomials

$$
c_{i}\left(r, \theta_{r}\right)=\sum_{i=0}^{\infty} \frac{y_{1}(r)}{r} r(\cos \theta),
$$




$$
f_{i}^{-}\left(\theta_{r}\right)=\sum_{l=0}^{x} f_{l}^{-} P_{l}\left(\cos \theta_{r}\right)
$$

where $y(r)$ is a regular solution of the equivalent radial equation (f.q. 4.3). and has the following (un-normalized) asymptotic form ${ }^{2}$

$$
y_{1}(r) \stackrel{r \rightarrow x}{\longrightarrow} a_{1} \sin \left(k \cdot r-\frac{1}{2} l \pi+\delta_{1}\right)
$$

We can then mateh the two symptotic forms of $r_{a}^{-}\left(r, \theta_{r}\right)$ to obtain $a_{t}$ and $f_{l}^{-}$. Then applying the spherical harmonic addition theorem to the Legendre polynomial gives for the stationary scattering wave (with incoming spherical wave) solution of 1.1. 4.1

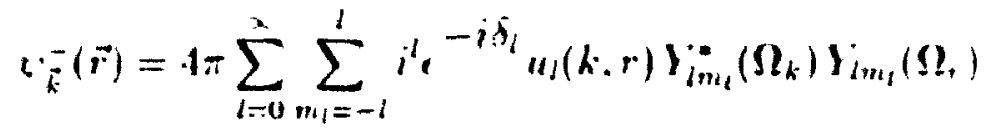

where $u_{l}(k .$,$) is the regular solution of Eq. 1.2 with ascmptotir form$

$$
u_{l}(k, r) \stackrel{r \rightarrow a}{\longrightarrow} \frac{1}{k r} \sin \left(k r-\frac{1}{2} l \pi+\delta_{l}\right)
$$

\subsection{The Fermi Approximation}

The simplest way to perform a correction due to FSI's is to use the Fermi approximation [35]. Wi assume that the FSl interaction is very short range. and hence that only the value at the origin of the relative wavefunction of the linal state particles is signilicant. Sime the wavefunct jon enters into the amplitude. the Si-wave cross-section would be enhanced hy a fartor of $\left|u_{0}(k, 0) / j_{0}(0)\right|^{2}=\left|u_{0}(k, 0)\right|^{2}$. and the cross-sections of otlier partial waves would be unaffected.

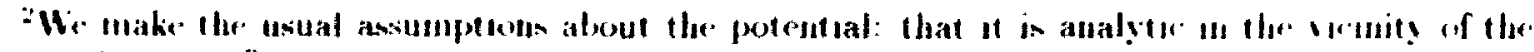
origin, that $\lim _{r \rightarrow 0}, \cdot v(r)=0$ and that $\lim _{r \rightarrow !} r(r)=0$. 


\subsection{Applying FSI's to the Flux-tube Breaking Model of Meson Decay}

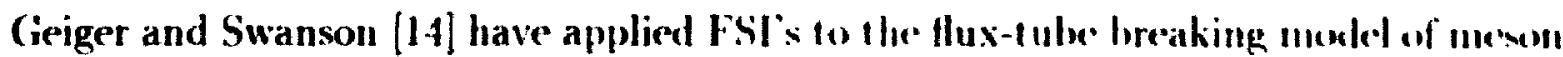
decay: Although we do not apply. their methodolog! in this work. we give the det dils here for completeness and because: it connects the two topies of this thesis (misum decay and FSI's). we are aware of no de taled publistied discussion of the me.thenl, allel our derivation includes factors neglected by Gerigur and Swallsull.

Recognizing that the last exponential function of Ey. 2.2.3 is the wavefunetient hetween the final state particles in the absence of an FSl potential (Fq. 1.11. Cieiger and Swanson replace it with the wavefunction in the preseme of such at perential

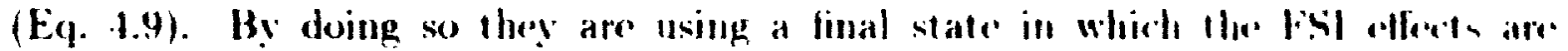
included. instead of simple plane waves in which the effects are igmored. This shomlal give a final result that includes the FSI effects.

Let us first examine the partial wave anplitudes from the llux-tulec breaking moded in the absence of FSl ss. In Section 2.4. wo lused the Jacol, Wiek formula to

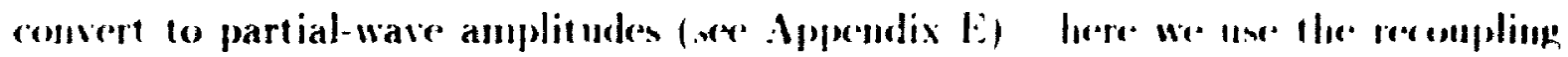

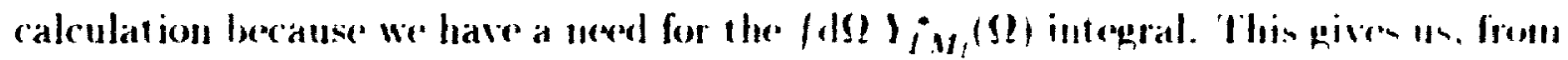
Eqs. 2.7 and 2.23. using Eqs. 2.2S and V..

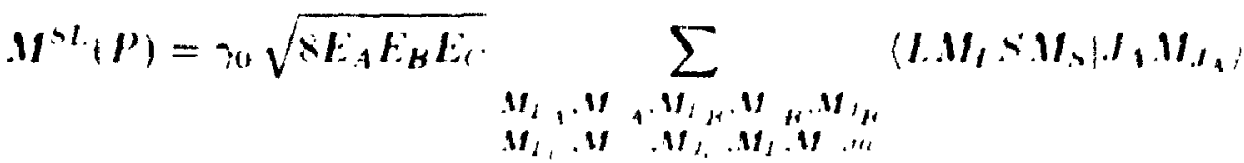

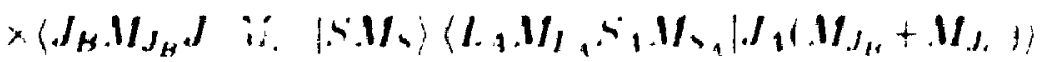

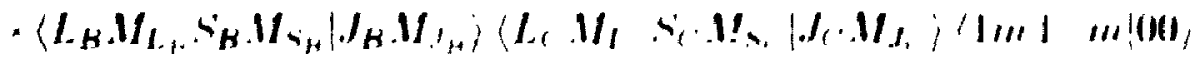

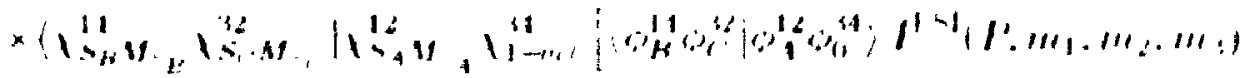

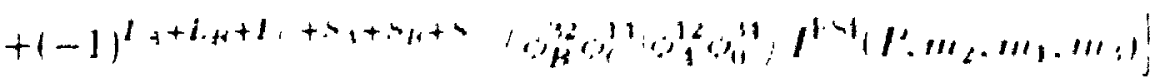


where

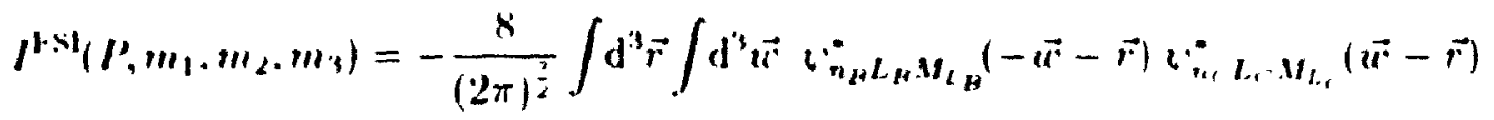

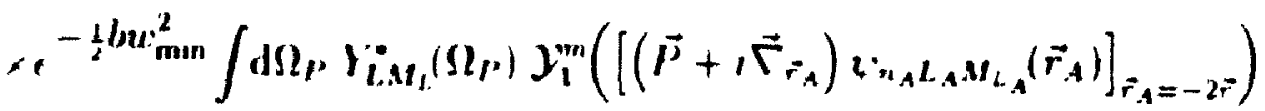

$$
\begin{aligned}
& x^{i \vec{p} \cdot(m+\vec{r}+m-\vec{w})} \text {. }
\end{aligned}
$$

Note that in evaluating $M^{s L}\left(J^{\prime}+\right.$ we can pick aily value of $M_{J_{4}}$ : altermatively. We could suIn cover $M_{I_{A}}$ and divide ly $\left(2, J_{A}+1\right)$. on the right side.

Next. sert

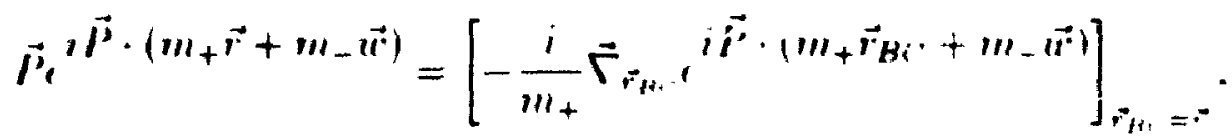

then wse an allermate form of by. 4.4 to set

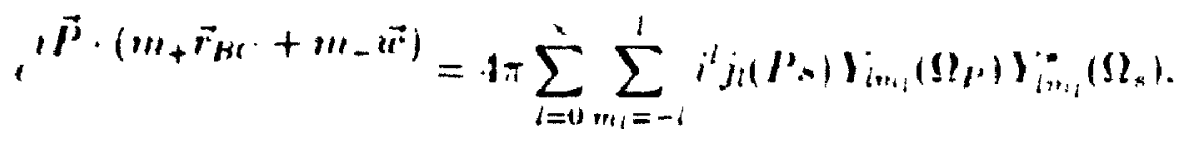

where $\vec{s}=m+\vec{r}_{B C}+m-\vec{k}$ and tinally apply the orthogonality of splerical harmonics lo ohluail

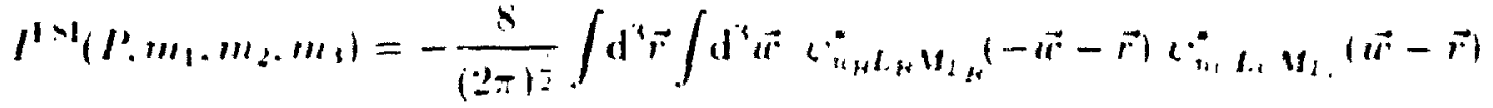

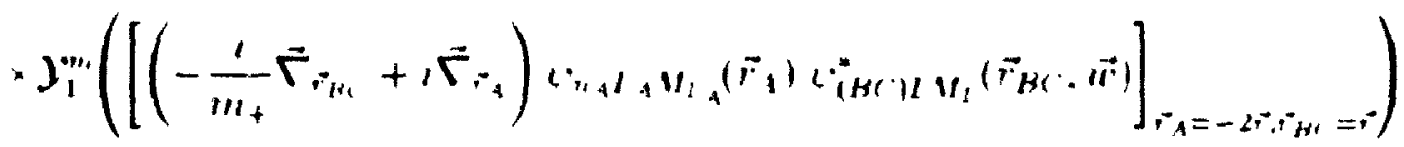

$$
\begin{aligned}
& x,-\frac{1}{2} b 112
\end{aligned}
$$

where. in the alesence of Fisls.

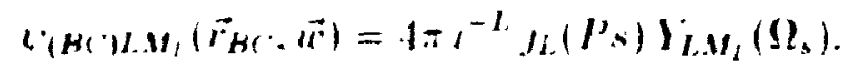




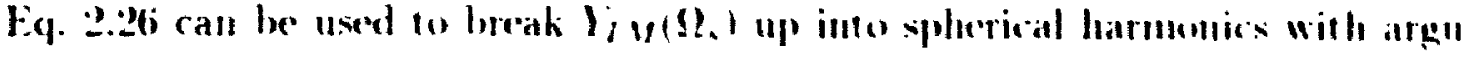
ments $\Omega_{\vec{r}}$, and $\Omega_{\text {n }}$ and of course. . is given hy.

$$
s=\sqrt{m_{+}^{2} r_{B C}^{2}+m_{-2}^{2} u^{2}+2 m+m-r_{B} \cdot \| \cos \theta}
$$

where $\theta$ is the angle lectwern $\vec{r}_{B i}$ and $a$.

The above is just another way of expressing the flux tube loreatimg menlel of meson decay of Section 2.3. Howerer. We can include the effoct of fith s ly replacines

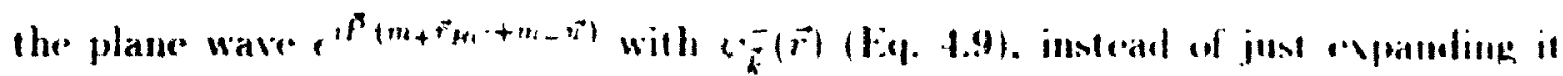
with Eq. 1.t. We then get. instead of Eq. I.lti."

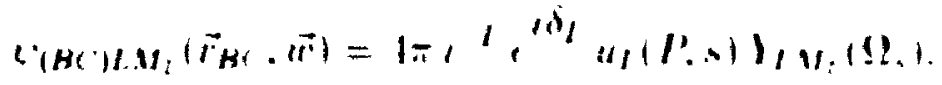

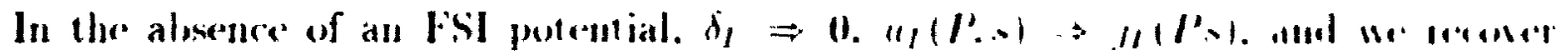
Eq. 1.16 .

\subsection{Applying FSI's to QED Amplitudes}

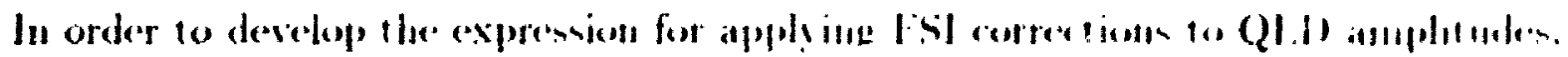

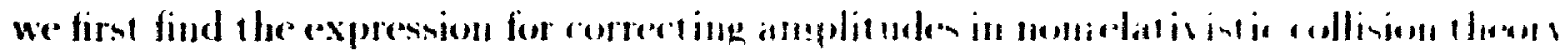

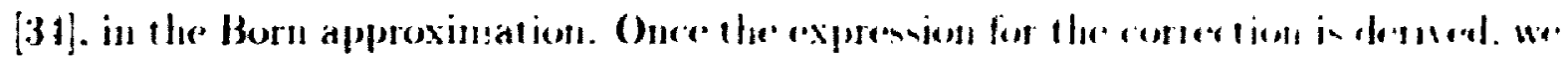

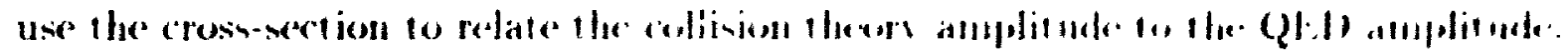

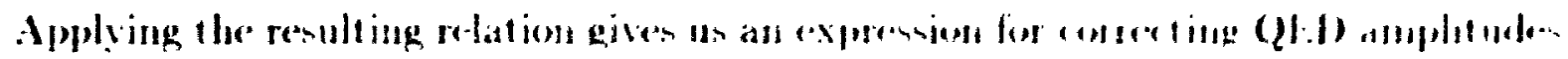

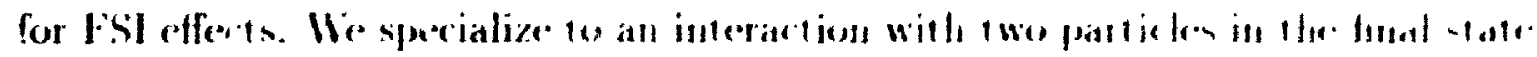

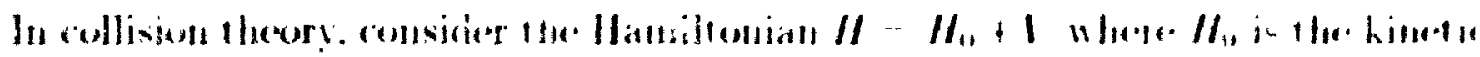

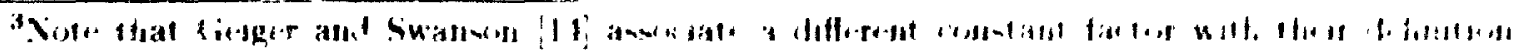
of cise \& 4 ( $\dot{\omega}_{L}$
} 
energy term. and ${ }^{\circ}$ is the potential. $1^{\circ}=1^{\circ}+t^{\circ}$ is divided into the potential representing the hasic interaction. If. and the FSI potential I. The plane wave $b^{\prime}(\vec{r})$ are the solutions of the Schrödinger equation in the alwence of a potential.

$$
\left(H_{1}-E\right) \phi_{k}(\vec{r})=0
$$

the stationary scattering waves $1 \frac{ \pm}{2}(\vec{F})$ are the solutions with the full potemtial.

$$
\left(I_{11}+I^{+}+I^{\prime}-E\right) V_{i}^{ \pm}(r)=0
$$

ald the stationary sattering waves $2 \cdot \frac{ \pm}{a}(\vec{r})$ are the solutions with the fol potential unls.

$$
\left(H_{0}+i-l\right) \cdot(\vec{r})=0
$$

- 1, $I_{h}^{2}(\vec{r})$ atre ilso solutions of the equivalent integral equation.

$$
I_{i}^{ \pm}=\left(1+\frac{1}{I-I_{1}-1 \pm 1: 1}\right) a
$$

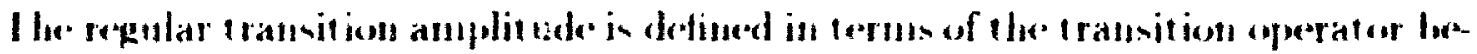

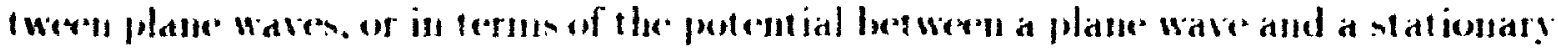
seatlering wate:

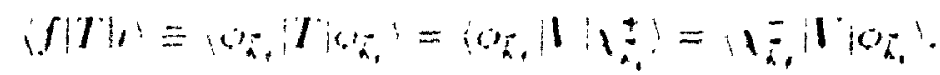

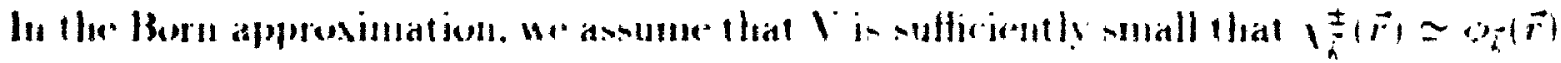

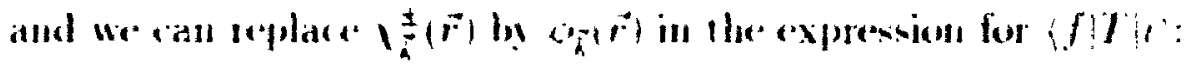

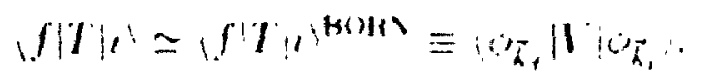




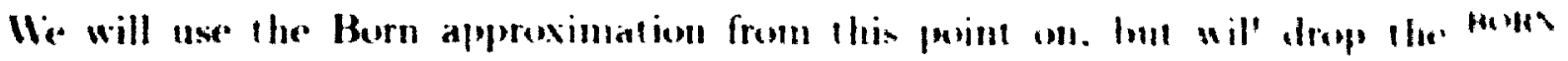
not ation for convenience.

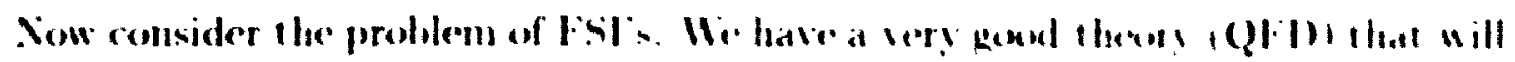

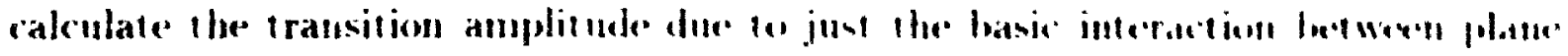

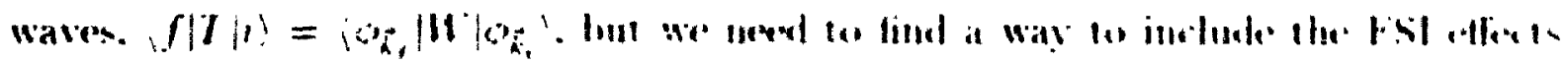

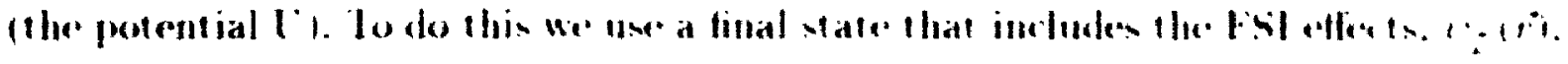

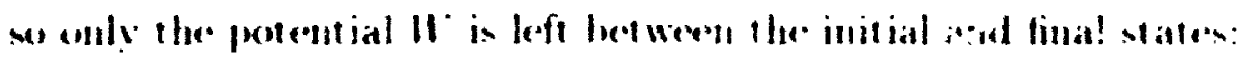

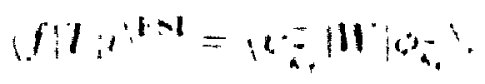

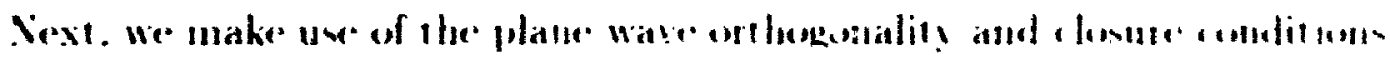

$$
\begin{aligned}
& \therefore b=12-1+1 \vec{k}-\vec{k} \cdot \\
& \frac{1}{12-1} \int d x+200-1
\end{aligned}
$$

to. : in: ain

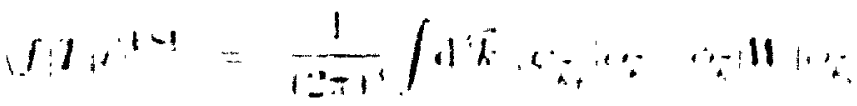

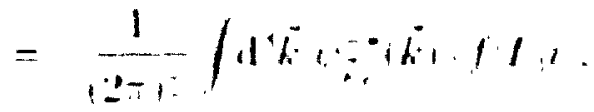

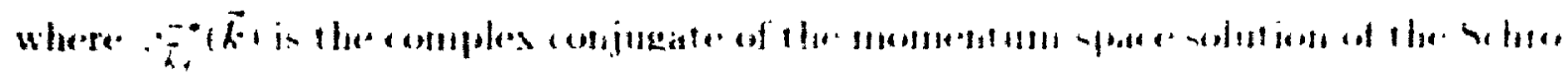

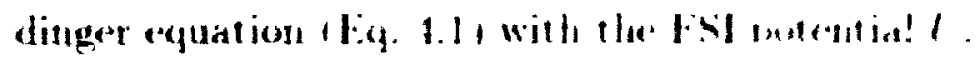

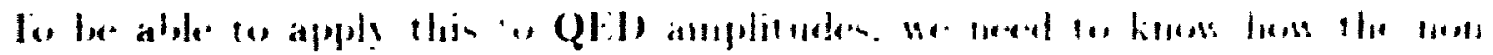

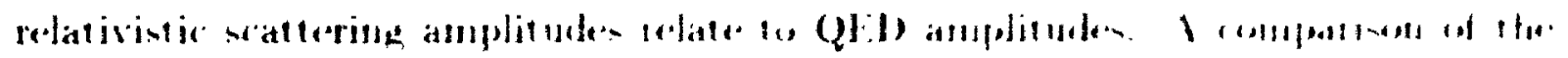

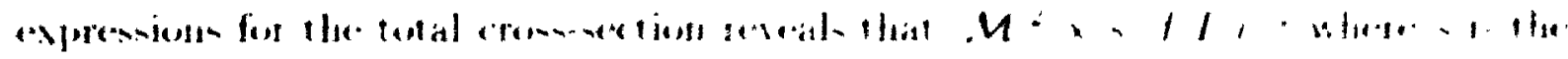

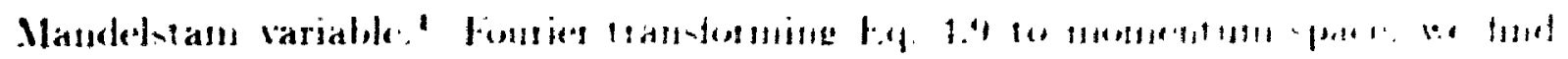

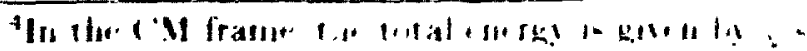


that the QHI) amplit.t tr corrected for FSI effects is

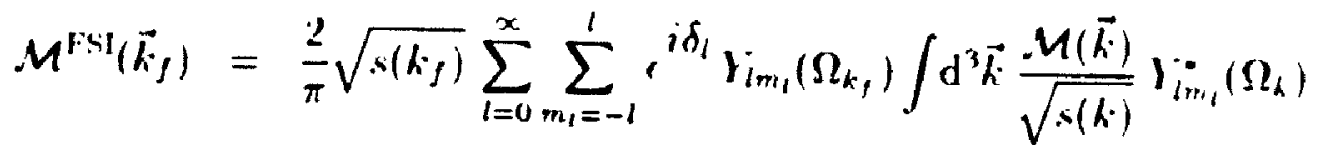

$$
\begin{aligned}
& \times \int_{0}^{x} \mathrm{~d} r r^{2} j_{l}(k r) u_{l}\left(k_{f} \cdot r\right)
\end{aligned}
$$

where a and $\mathcal{M}$ have bren written as functions of the momenta in order to show whether they are calculated with the variable of integration, $\vec{k}$. or the relative momentum between the final state particles in the (M frame. $\vec{k}$. There is ambiguity as to how to relate $k$ and $s(k)$ nouclativistically or relativistically. Wr follow the usual practice of keeping as much as possible relativistic. and so use for regual-mass final state parti its: $s(k)=4 k^{2}+4 m^{2}$.

It is worthwhile to expand each. $M$ into partial waves.

$$
M(\vec{k})=\sum_{L=0}^{\infty} \sum_{M_{l}=-L}^{L} f_{L, M_{l}}(\cdot(k)) Y_{L, M_{L} \mid}\left(\Omega_{k}\right)
$$

in order to get the FSl correction for a single partial wave

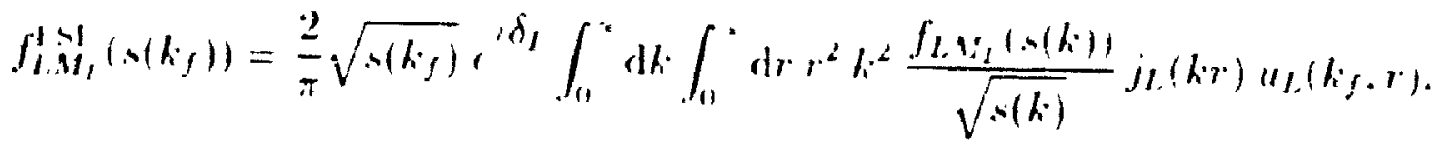

In the absence of an FSI potential. $\delta_{l} \Rightarrow 0 . u_{L}\left(k_{f}, r\right) \Rightarrow j_{L}\left(k_{f} r\right)$. the orthogollality condition ai he spluerical Bescel functions gives us a delta function. and wo obtain

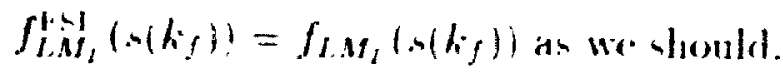




\section{Chapter 5}

\section{Final State Interactions: An}

\section{Application}

\subsection{The Interaction $\gamma \gamma \rightarrow \pi \pi$}

In 1986. a group at Orsay (using DMI on DC(I) measured the cress section of in $\rightarrow$ $\pi^{+} \pi^{-}$near the $\pi \pi$ threshold. and found a cross-section abeut twice that experded

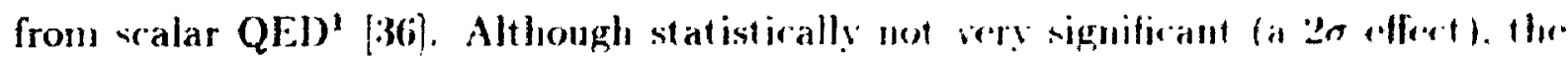
result aroused interest because it differed from theoretical experctations. By. Low sow energy theorem [3i] the interaction of low energy photom depende only on the stat ie properties of the target. Becanse at low energies the longe wavelenglh photend atmot

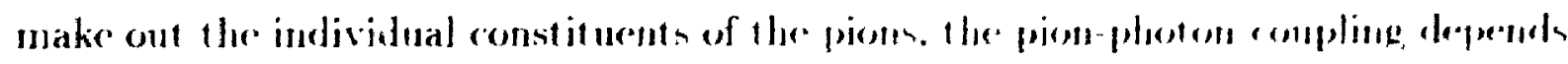

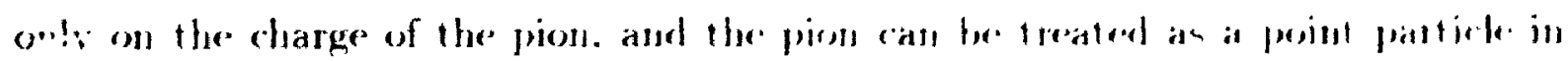

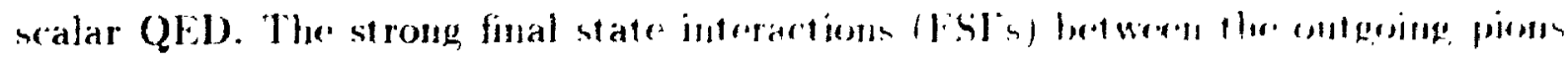
were not expected to be as signilicant an the electrowerate efferce.

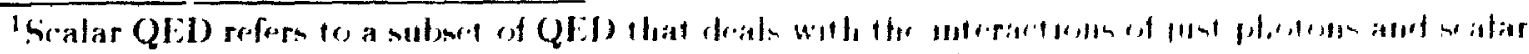

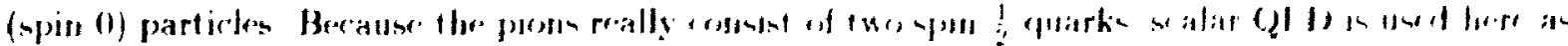

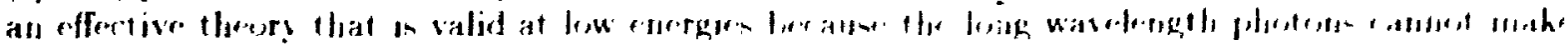
out the mblividual quark-
} 


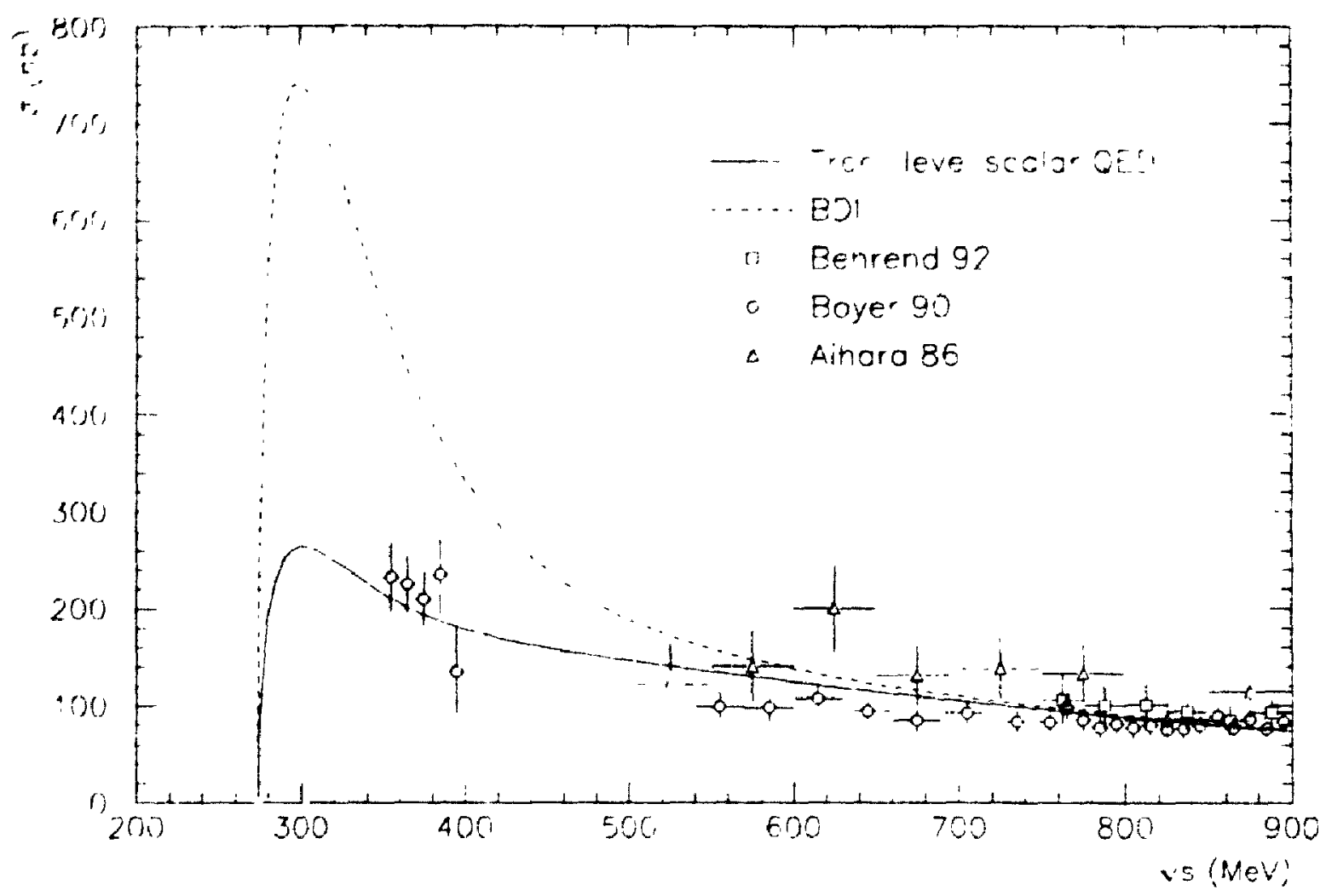

Figure 5.1: (iraph of the $\gamma \gamma \rightarrow \pi^{+} \pi^{-}$total cross-section is. $\sqrt{s}$, for the tree-level scalar QEI) and BDI predictions (reproduced by :s). and recent data. The curves have been corrected to have a limited polar arceptance to match the data: $|\cos \theta| \leq 0.6$. The data is from Behrend ef al. [38]. Hover $f$ al. [39] and Aihara $f$ al. [40]. Some' of the data shown in this chapter was obtained by us from References [41] and [12]. The horizont al error hars on the data show the hin sizes; for the vertical error bars all of the given errors were added together in quadrature.

In 1987. Barnes. Dooley and Jigur (BD) ) [13] estimated the effects of thene Fil's using the Fermi approximation (see Section 4.3 ) with an effective potential ext racted from a quark model, and found a similar enhancement. Infortunately. the experimental enhancenent has since disappeared: the early experiments were apparently plagued by problems with subtracting the lepton background duc to low statistics [1.1]. and the later experiments show no suchenhancement. Figure 5.1 shows the two curves and the latest experinental data. Note that the data fits the tree-level scalar Qlil) curve well for the whole range shown (up to a ('M energy of 900 Mel). even 
though we would not necessarily expect it to be accurate at the higher energices.

Calculations of the $\gamma \rightarrow \pi \pi$ cross-sections have becol done using of her methods. One-loop chiral perturbation theory has produced mixed results: the is $\rightarrow \pi^{+} \pi^{\text {- }}$ cross-section curve [45] shows a small enhancement over the tree-level sialar (Qlil) curve near threshold that is still compatible with the latest experimental diti. While. the $\gamma \gamma \rightarrow \pi^{0} \pi^{0}$ cross-section curve $[45,16]$ has a shape that is rather different from the data. However, a two-loop chiral perturbation theory calculation of the $7 \rightarrow \rightarrow " \pi$, cross-section [47] agrees with the data. The difference is apparently mainly due to $\pi \pi$ rescattering and renormalization of the pion decay constant.

Calculations of the $\gamma \rightarrow \pi \pi$ cross-sections bave also bern done with dispersion relations. typically using phase shift data from $\pi \pi$ scatlering. and constraints from unitarity. analyticity and crossing. The $\urcorner 9 \rightarrow \pi^{+} \pi^{-}$cross-section results are agaill quite similar to both the tree-leve! scalar QEI) curve and the experimental data meat threshold [48. 49]. The $\gamma \gamma \rightarrow \pi^{0} \pi^{0}$ cross-section result also de well |14. 19]. showing good agreement with the data. It is bedieved that higher order exchange offerts. whose absence is problematic for the one-loop chiral perturbat ion theory ralo alation. are included in the dispersive calculation through the mitarity mequerement | 19 ].

On the face of it, the failure of the quark model to do as well as these of hee twe approaches represents quite a blow to it meputation. Being able to prodict llie effects of FSI's is inportant if we are 10 mulerstand $\left.(2)^{\circ}\right)$. In adedition. if we understend the

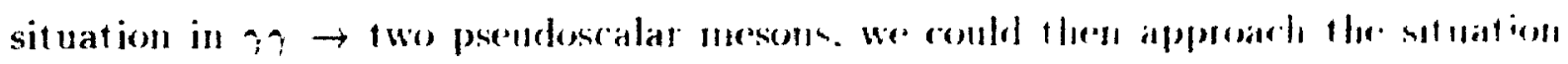

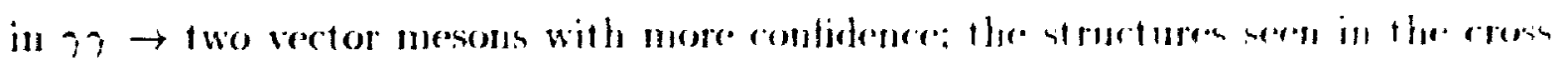

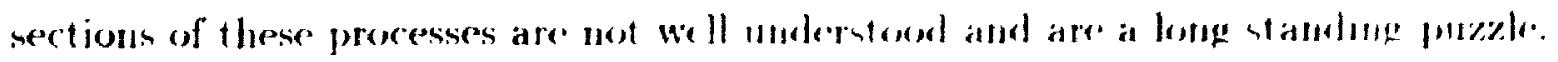

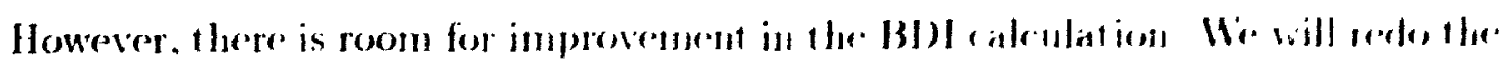

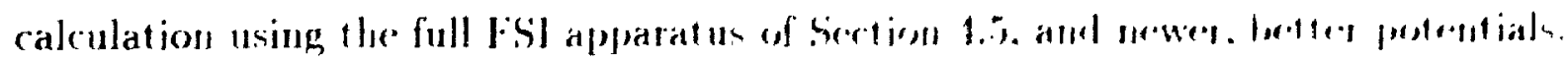

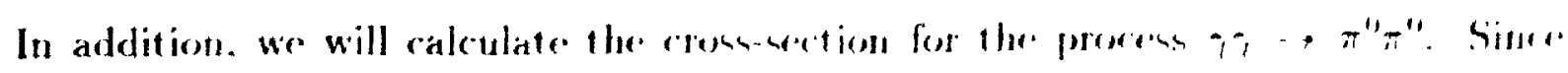

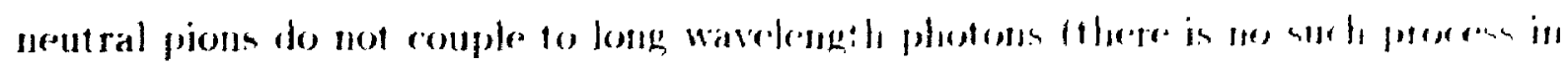




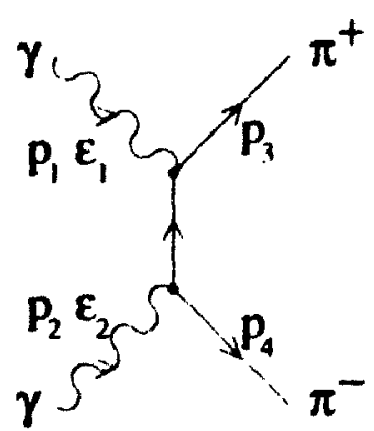

(a)

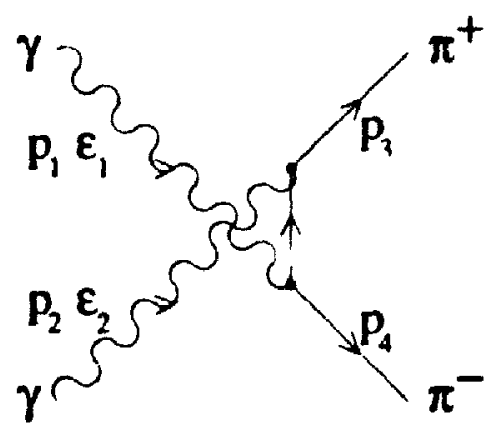

(b)

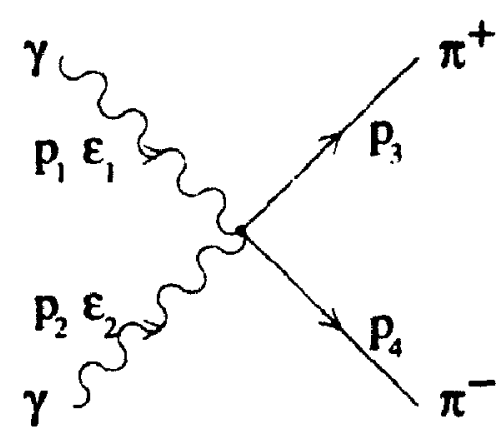

(c)

Figure 5.2: The tree-level Feynman diagrams for the scalar QED interaction in $\rightarrow$ $\pi^{+} \pi^{-}$. The labels of the particle momenta $\left(p_{1}\right)$ and photon polarization vectors $\left(c_{2}\right)$ are shown.

scalar QED), $\gamma \gamma \rightarrow \pi^{0} \pi^{0}$ is expected to be suppressed relative to $\gamma \rightarrow \rightarrow \pi^{+} \pi^{-}$making the effects of the FSI's relatively much more significant. In our calculation. it is the FSI potentials that mix the $\pi^{+} \pi^{-}$final state into $\pi^{0} \pi^{0}$.

In Section 5.2 we calculate the cross-section for $\gamma \rightarrow \pi^{+} \pi^{-}$in tree-level scalar QEDI. In Section 5.3 we discuss the effective potentials and the application of the full FSI apparatus (see Section 4.5) to this problem. In Section 5.1 we give the results of our calculations.

\section{2 $\gamma \gamma \rightarrow \pi^{+} \pi^{-}$in Tree-level Scalar QED}

The three trex-level scalar QEL) Feynman diagrams" cont ributing to the interaction $\gamma \rightarrow \pi^{+} \pi^{-}$are shown in Figure 5.2.

The corresponding Feymman amplitude (using the momenta and photon polarization labels shown in Figure 5.2) is

$$
M=4 \pi a\left[\frac{e_{1} \cdot\left(2 p_{3}-p_{1}\right) c_{2} \cdot\left(2 p_{4}-p_{2}\right)}{\left(p_{2}-p_{4}\right)^{2}-m^{2}}+\frac{c_{1} \cdot\left(2 p_{4}-p_{1}\right) c_{2} \cdot\left(2 p_{3}-p_{2}\right)}{\left(p_{1}-p_{4}\right)^{2}-m^{2}}+2 t_{1} \cdot \epsilon_{2}\right]
$$

\footnotetext{
Ster Appendex A 2 for a brief explanalen of leyman diagrams
} 


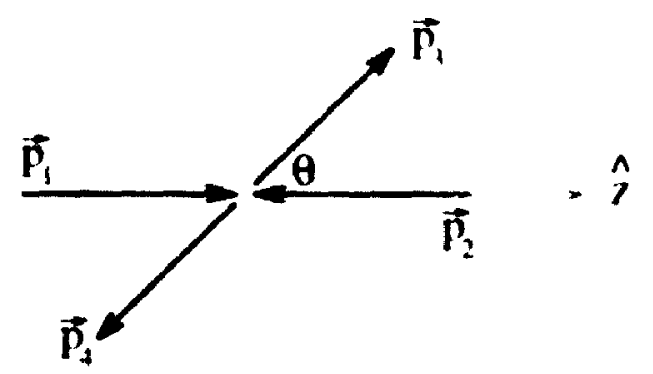

Figure 5.3: The geonetry of the $79 \rightarrow \pi^{+} \pi^{-}$interaction in the ('M frante.

where $a$ is the fine structure constant and $m$ is the pion mass. Areraging the photon polarizations and integrating over phase space gives us the total cross-rection

$$
\sigma=\frac{\pi a^{2}}{4 m^{2}}\left[2 x(1+r) \sqrt{1-x}-r^{2}(2-r) \ln \left(\frac{1+\sqrt{1-x}}{1-\sqrt{1}-x}\right)\right]
$$

where $r \equiv \frac{4 m^{2}}{\$}$ and $s$ is the Mandelstam variable.

We would now like to work out the cross-sections corresponding to dilferent peatial waves in the final state. In the ('M frame, we take $\vec{p}_{1}$ ter print in the pusitive: direction. and the direction of $\vec{p}_{t}$ to be described by the spherieal pelate condinates $\theta$ and 0 , as shown in Figure 5.3 . For the photons we take circular polarizatiens:

$$
\begin{aligned}
& c_{1}(+)=c_{2}(-)=\frac{1}{\sqrt{2}}(1) \cdot 1 \cdot-1 .(1) . \\
& \left.\left.c_{1}(-)=c_{2}\right)+1=\frac{1}{\sqrt{2}}(0.1 . \cdots 1.1)\right)
\end{aligned}
$$

We calculate the helicity ampliturlen

$$
\begin{aligned}
& M_{++}=. M_{-}=\frac{n \pi a r}{1-11-x) \cos ^{2} \theta} .
\end{aligned}
$$

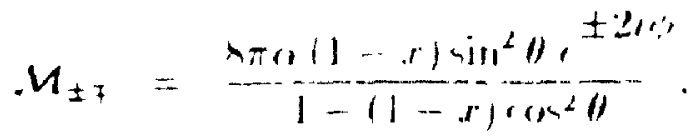


and then expand them in a spherical harmonic basis to obtain the partial waves (e.g. for the + + case $)$.

$$
\begin{aligned}
& M_{++} \equiv \sum_{L=1}^{\infty} \sum_{M_{L}=-I .}^{L} f_{L M_{L}}^{++} V_{L M_{t}}(\theta . o) \\
& \Rightarrow \quad f_{L M_{t}}^{++}=\int \mathrm{d} \Omega V_{i M_{1}}(\theta, \phi) M_{++}
\end{aligned}
$$

We lind that $f_{L M_{t}}^{++}=f \bar{M}_{L}$ is only non-zero for $M_{L}=0$ and that $f_{L, M_{L}}^{+}=f_{L-M_{L}}^{++}$is only non-zero for $M_{L}=2 .{ }^{3}$ The cross-sertion is then written in terms of the partial waves as

$$
\sigma=\frac{\sqrt{1-r}}{12 \pi \pi^{2}, a}\left[\left(f_{00}^{++}\right)^{2}+\sum_{L \geq 2,+\mathrm{vent}}^{2}\left[\left(f_{l, 0}^{++}\right)^{2}+\left(f_{l, 2}^{+-}\right)^{2}\right]\right] .
$$

The nerded partial waves (see below) are given bs

$$
\begin{aligned}
& f_{(x)}^{++}=i \pi \frac{x}{2} \frac{x}{\sqrt{1-x}} \ln \left(\frac{1+\sqrt{1-x}}{1-\sqrt{1-x}}\right) . \\
& f_{21}^{++}=1 \sqrt{5} \pi^{\frac{3}{2}} \alpha \frac{x}{1-x}\left[-6+\frac{2+x}{\sqrt{1-x}} \ln \left(\frac{1+\sqrt{1-x}}{1-\sqrt{1-x}}\right)\right] \text {. } \\
& f_{22}^{+-}=\sqrt[4]{\frac{15}{2}} \pi \div 0\left[\frac{10}{3}-\frac{2}{1-x}+\frac{x^{2}}{(1-x)^{\frac{2}{2}}} \ln \left(\frac{1+\sqrt{1-x}}{1-\sqrt{1-x}}\right)\right] \text {. } \\
& f_{4+1}^{++}=3 \pi^{3} \times \frac{x}{1-x}\left[\frac{110}{3}-\frac{70}{1-x}+\frac{3 r^{2}+24 x+8}{(1-x)^{3}} \ln \left(\frac{1+\sqrt{1-x}}{1-\sqrt{1-x}}\right)\right] \text {. } \\
& f_{i}^{+-}=3 \sqrt{10} \pi \pi^{2}\left[\frac{-54}{5}+\frac{i 6}{3(1-x)}-\frac{14}{(1-x)^{2}}+\frac{x^{2}(6+x)}{(1-x)^{\frac{3}{2}}} \ln \left(\frac{1+\sqrt{1-x}}{1-\sqrt{1-x}}\right)\right] \text {. }
\end{aligned}
$$

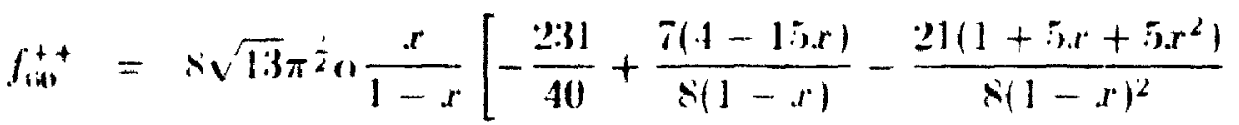

$$
\begin{aligned}
& \left.+\frac{16+190 x+9(1) x^{2}+5 x^{3}}{16(1-x)^{\frac{1}{2}}} \ln \left(\frac{1+\sqrt{1-x}}{1-\sqrt{1-x}}\right)\right] \text {. }
\end{aligned}
$$

The total cross-section and the crose-sections of the $l=0.2 .1$ partial waves are

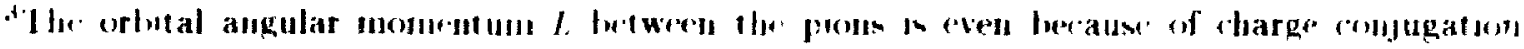
intrathe
} 


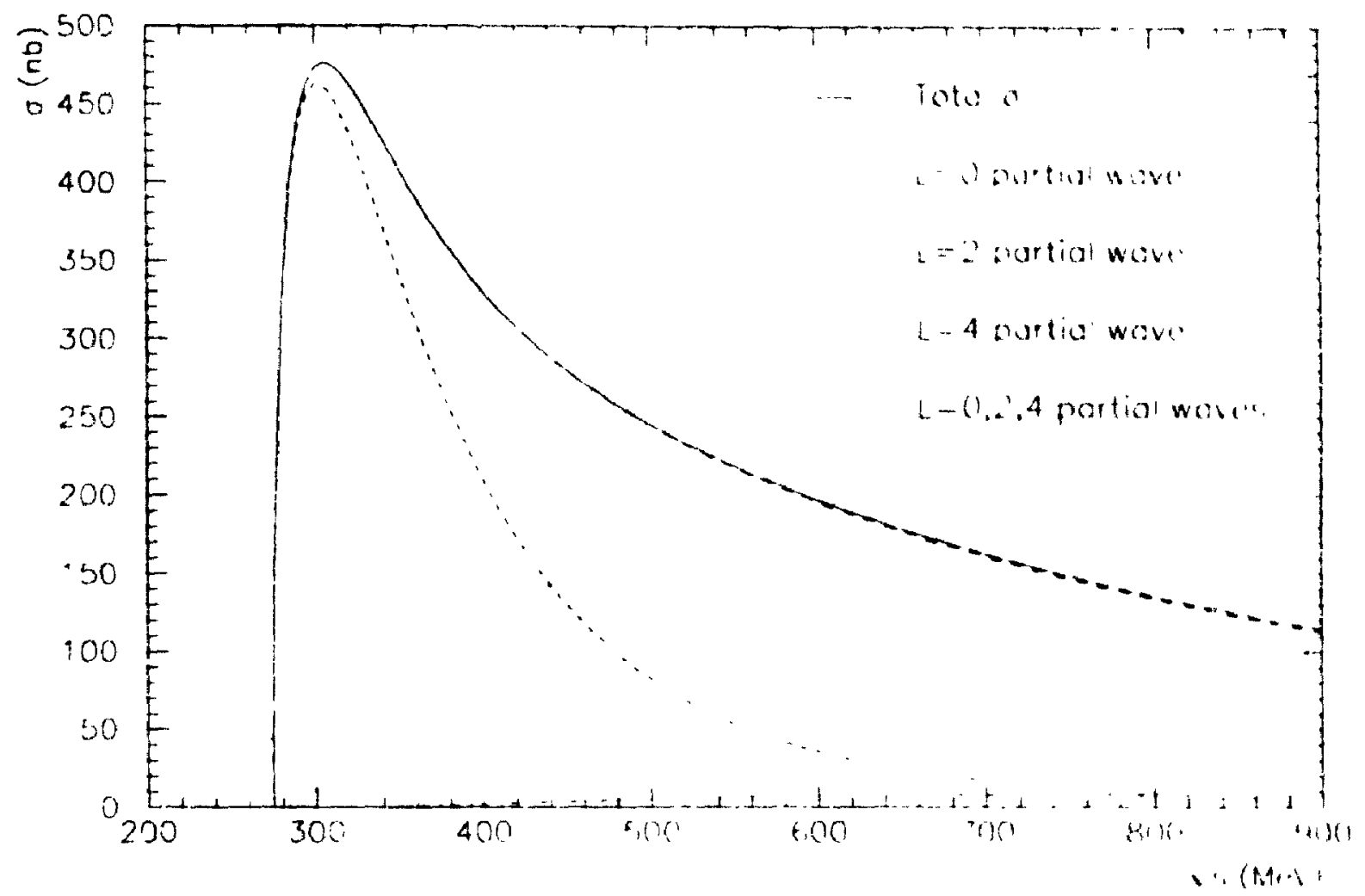

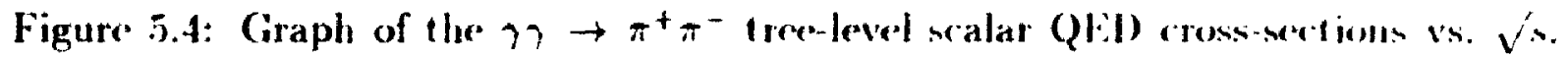
The total cross-section is shown, as well as the cross-sections of the $l=11.2 .1$ partial waves. and the cross-section corresponding to the sum of the $I=0.2 .1$ partial waves. The curves are for full polar acceptance: $|\cos \theta| \leq 1$. which is why they ditler from those shown in Figure 5.1.

shown in Figure:5.1.

\subsection{Applying the FSI Correction}

\subsubsection{The Effective Potentials We Use}

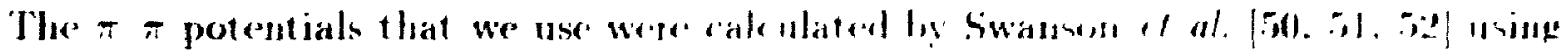

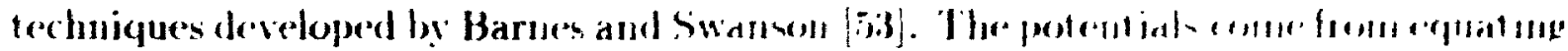

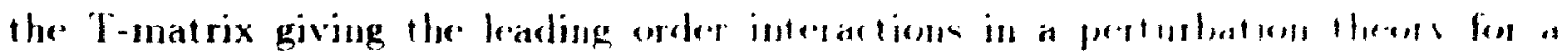

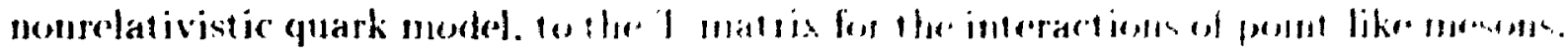




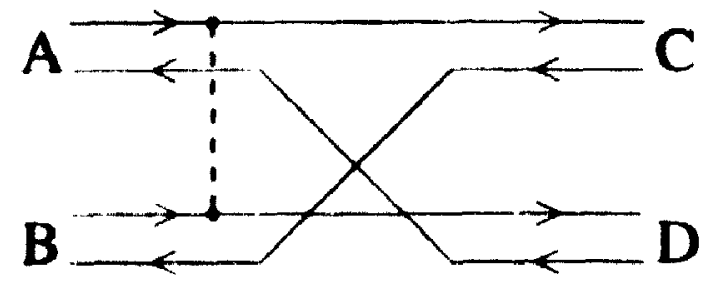

a)

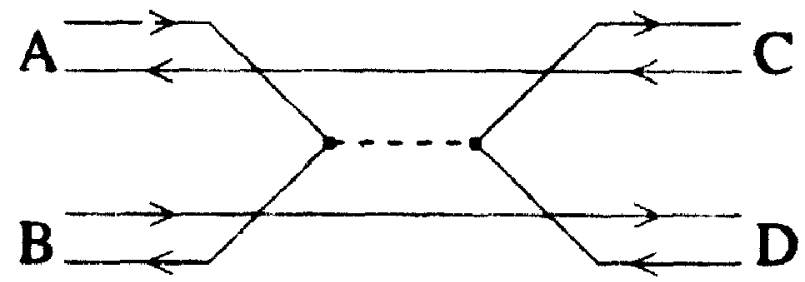

b)

Figune 5.5: Lxample diagrams of the two classes of interactions included in the quark model used to find the effective potentials: a) t-channel gluon exchange. b) s-channel gluon exchange.

writ!en in terms of the eflective potential they were trying to find.

'The potentials are different for the different isospin states of the two pions. Berauss of charge conjugation invariance. the orbital angular momentum $l$. between the I wo pions must be even. Because of Bose-Einstein symmetry (which holds for mermbers of the sane isospin multiplet in the context of the strong interaction [54]). the sum $L+I$ must be event, and hence the total isospin $I$ must be esen as well. II then follows that the isospin states (written as $\left.\left|I I_{z}\right\rangle\right)$ of the pions are

$$
\begin{aligned}
\left|\pi^{+} \pi^{-}\right\rangle & =\sqrt{\frac{2}{3}}|00\rangle+\frac{1}{\sqrt{3}}|20\rangle . \\
\left|\pi^{\prime \prime} \pi^{0}\right\rangle & =-\frac{1}{\sqrt{3}}|00\rangle+\sqrt{\frac{2}{3}}|20\rangle .
\end{aligned}
$$

There are two classes of interaction included in the quark model used to find the affective potentials. The first, referred to as 1-channel gluon exchange by Swanson it al.. involves both one-gluon-exchange and a linear confining potential. followed by quattk rearrangetnent lack into colour singlet states. This can occur for hoth $I=0$ and $l=2$ states. The second. referred to as s-channel gluon exchange. involies the annililation of two of the quarks. producing a menon hybrid in the intemediate state. Because of the internediate state, this interaction can only occul for $I=0$ states. Fxample diagrams of the two classes of interaction are shown in Figure 5.5. 
We only present results for $F i l$ corrections to the $l=0$ partial wave of , ? because the $L=2$ potentials available from the sance sources are smaller than thowe for $L=0$. and we found that their effect on the cross-section was megligihle.

Although relativistic phase space is included in the quark medel used for lind the effective potentials, the potentials themselves are essentially nourelativistic. It :s our hope that they will contain emough phỵsics to correctly calculate the disturtion of the final state wavefunction leading to Fsil effects. However, it is known [5it. 5i3. iul] that the 1 -channel gluon exchange potential is mable wo accurately predict the plass. shifts resulting from $I=2 \pi \cdot \pi$ scattoring. apparently beratuse it lacks rolativistia phase space. It is expected that the other potentials sutfer from the same problent.

Weinstein and Isgur [5.5] have argued that this is related to the imalility of the nonrelativistic quark model to accuratel! pedict the charge madii of the ground stalle

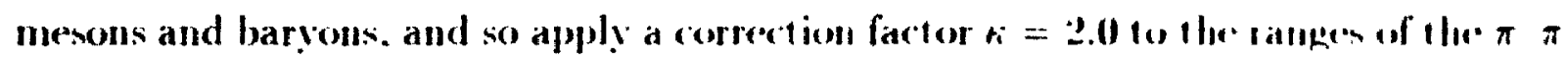
potemtials. and an overall normalization factor $\eta=0.6$ which is litted lo rexpriment. 'To he specific, a potentiai riginally parametrized h!

$$
I(r)=1,-\frac{1}{1}\left(\frac{1}{1}\right)
$$

is writtell as

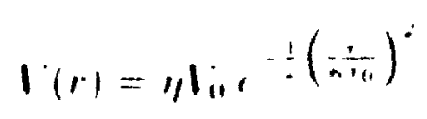

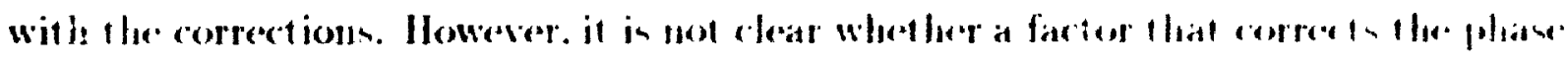

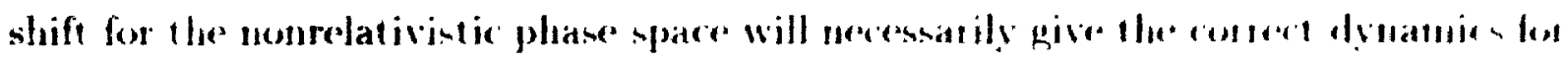

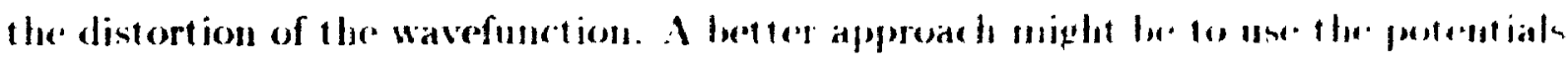

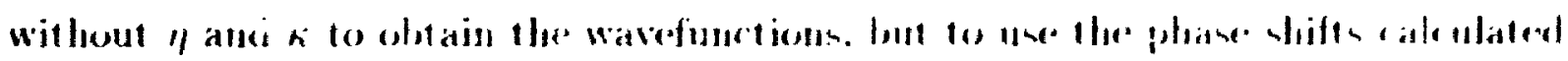

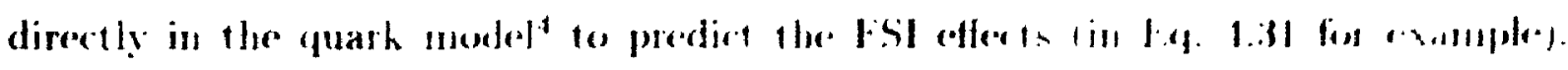

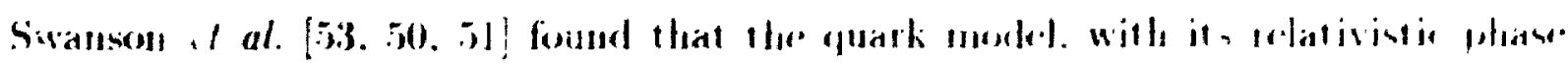

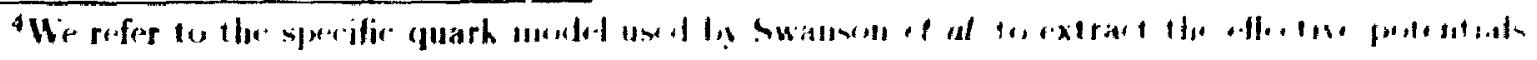




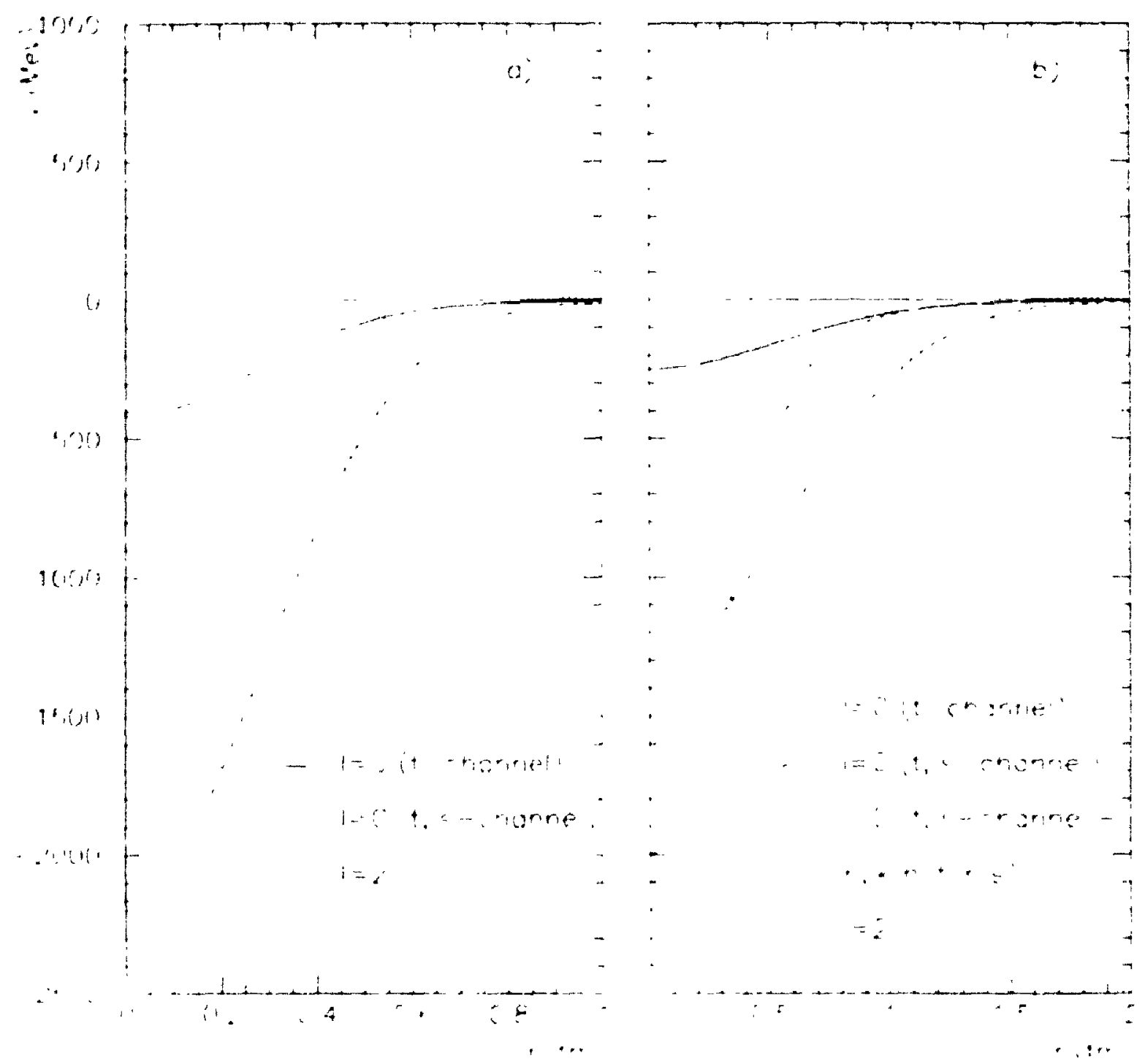

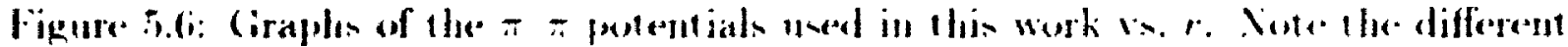
scales on the two axes. I - hannel gluen exchange is present in all pesentials. Fon $l \approx 0$. the potentials are plotted beth with and without the contribution fromsdiannel gluen exchange. In al. the perential- without the factors $1 /$ and to ate protted.

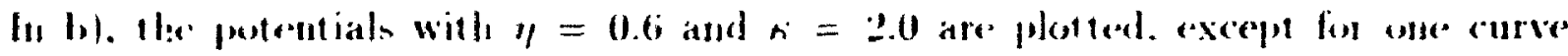
where the cont ribution from t-channel gluen exchange contains $y$ and th. but that

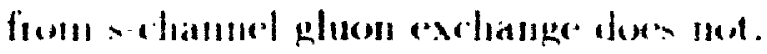

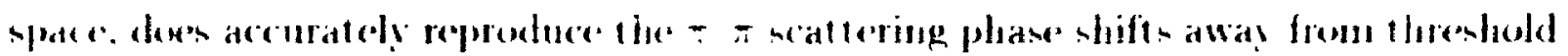
(allheugh resonance eflects mun les added for $l=0$ see below).

In addition. since $1 /$ and a has periously bests apphed only to potentiats repe- 


\begin{tabular}{|c|c|c|c|c|c|c|c|}
\hline \multirow{2}{*}{\multicolumn{2}{|c|}{ Pion Sitate }} & \multicolumn{6}{|c|}{ 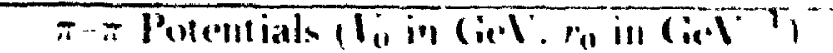 } \\
\hline & & \multicolumn{4}{|c|}{ t-chanmel gluon exchange } & \multirow{2}{*}{\multicolumn{2}{|c|}{$\begin{array}{l}\text { s-ahatmind } \\
\text { glums exchatiges }\end{array}$}} \\
\hline \multirow[b]{2}{*}{ l. } & \multirow[b]{2}{*}{1} & \multicolumn{2}{|c|}{ hypertine } & \multicolumn{2}{|c|}{ contimensent } & & \\
\hline & & $T_{11}$ & $r_{11}$ & Ti: & $r_{0}$ & $I_{11}$ & $r$ \\
\hline 0 & 0 & $-(11.349$ & 1.316 & $-(1) .1) ! 1$ & $2.2 ! 1$ & -1.7. & I. \\
\hline () & $\because$ & $0.7 i t i$ & $\mathbf{i} .31$ & $0.11 \%$ & $2.2 ! 1$ & & \\
\hline 2 & $\theta$ & -0.411. & 1.10 & $0.011 \%$ & 1.19 & & \\
\hline ? & 2 & $0.0 \times 5$ & 1.411 & $-(9.0 .3 \%$ & 1.19 & & \\
\hline
\end{tabular}

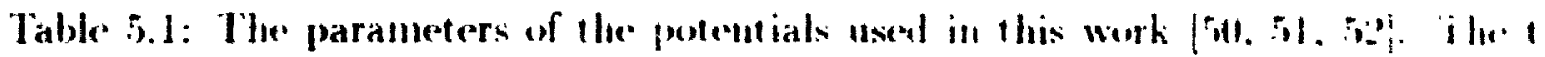

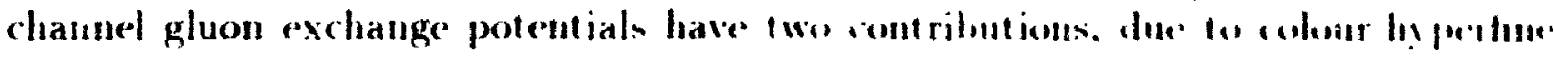

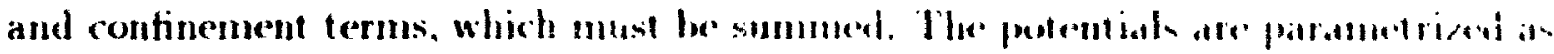

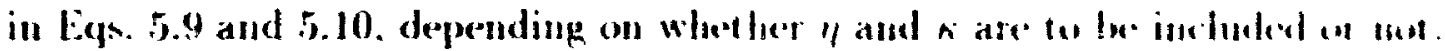

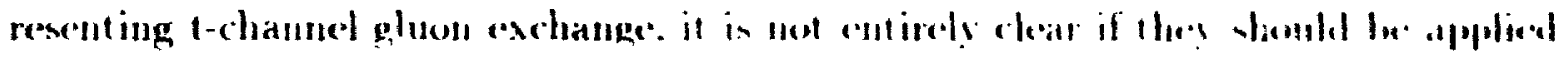

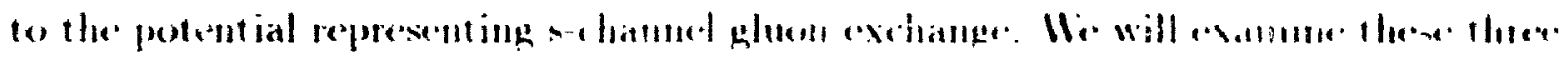

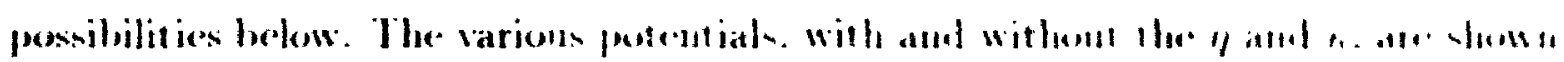

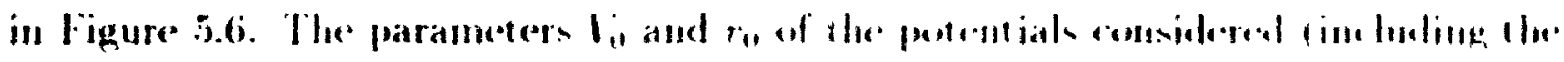

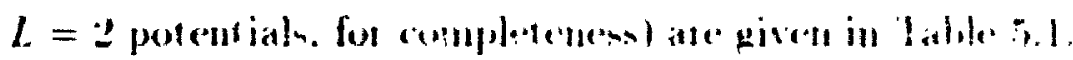

\subsubsection{The FSI Apparatus Applied to the Pions}

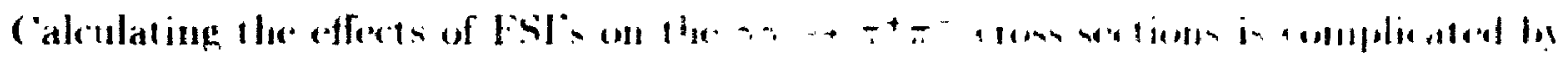

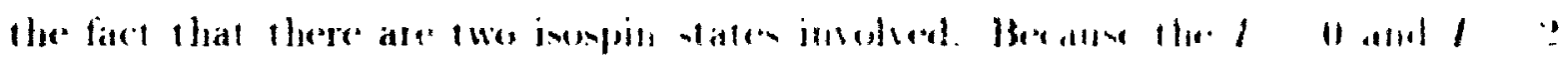

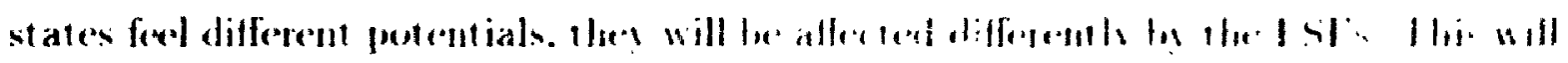

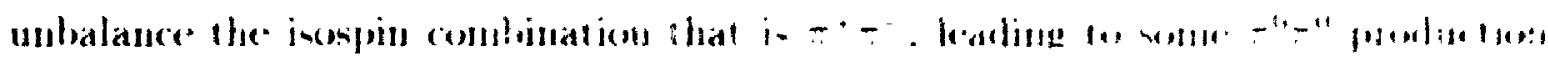

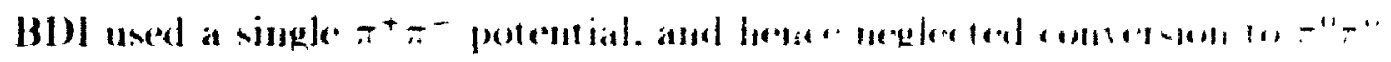

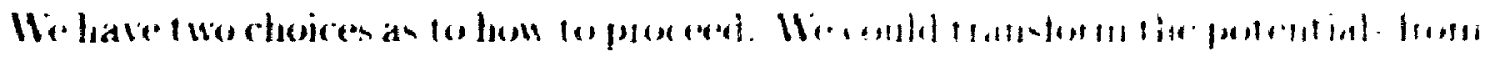

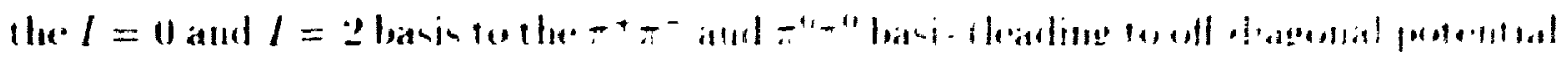

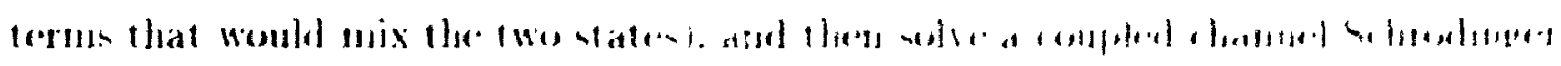

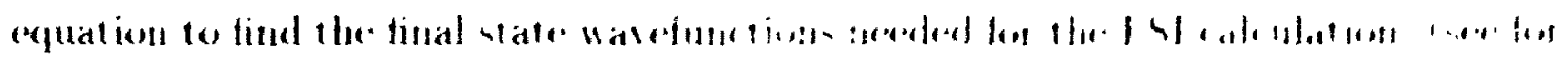

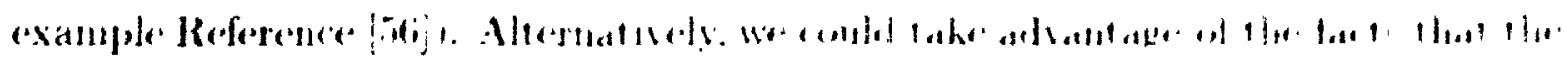




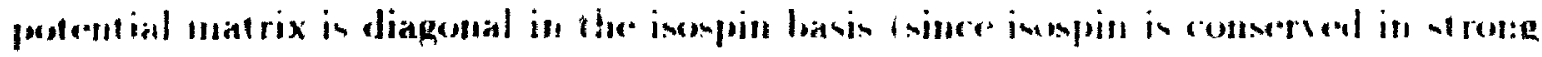

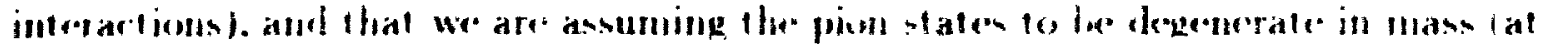

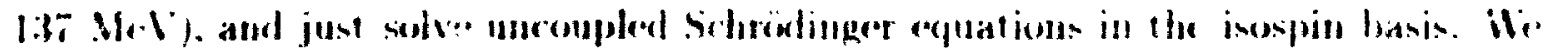
opl for this seremel iliuice.

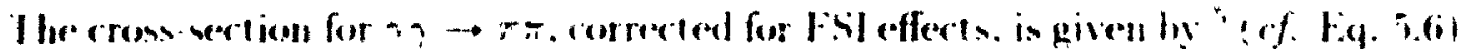

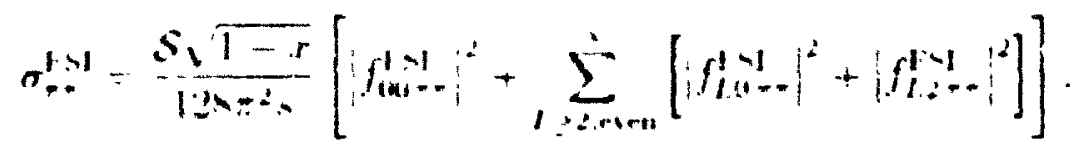

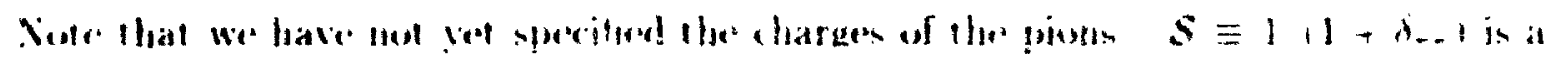

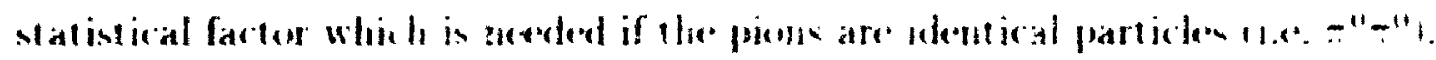

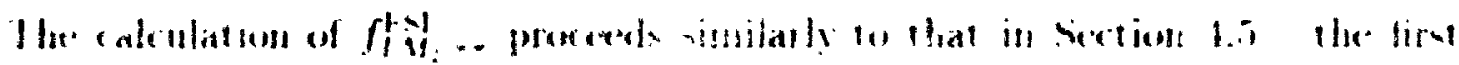

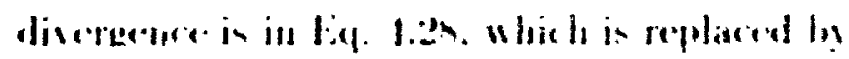

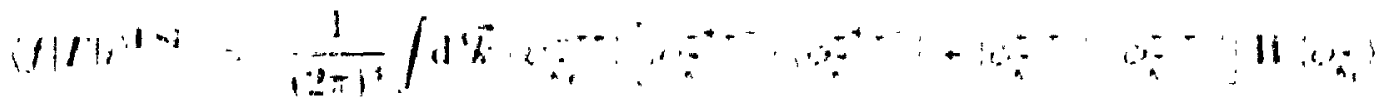

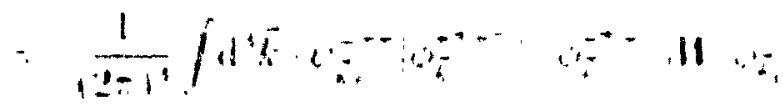

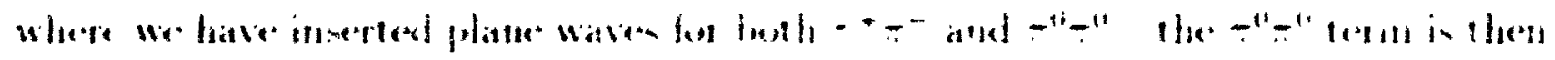

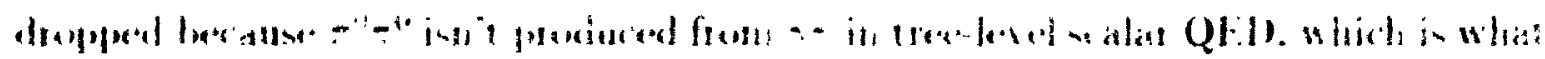

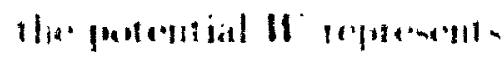

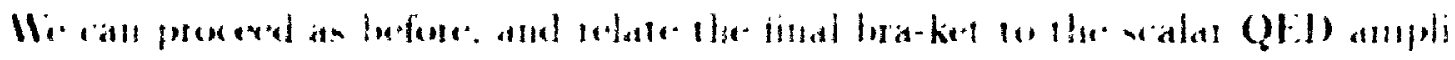

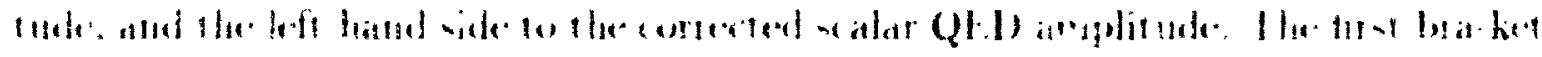

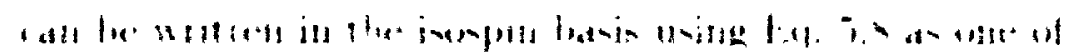

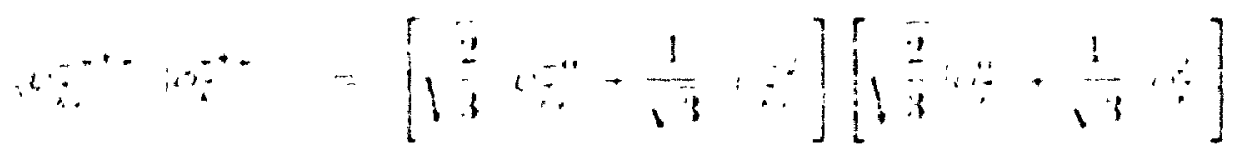

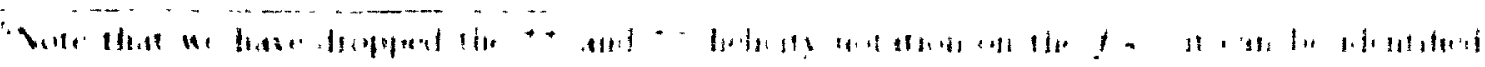

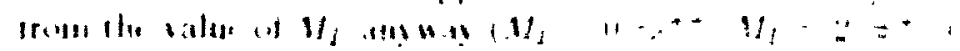




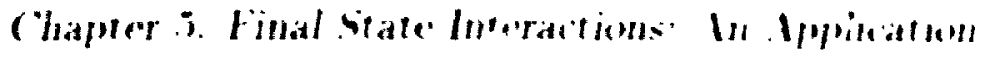

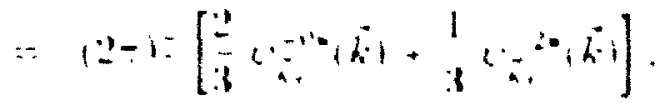

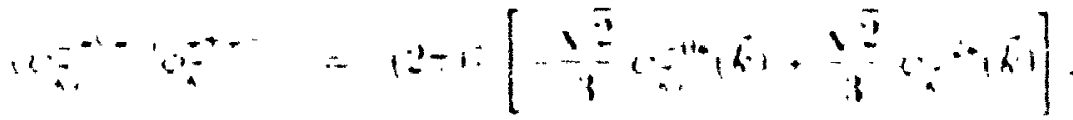

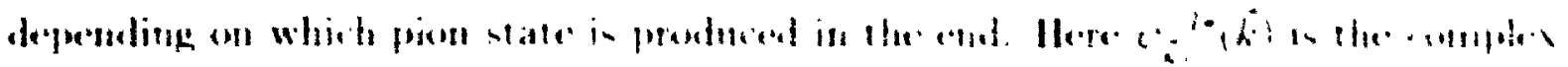

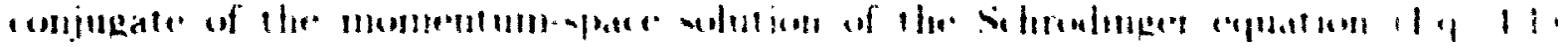

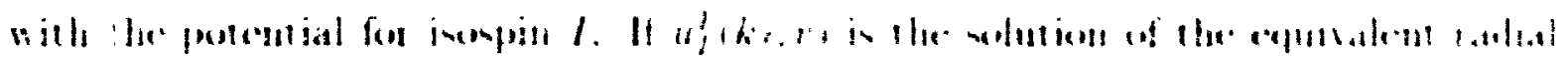

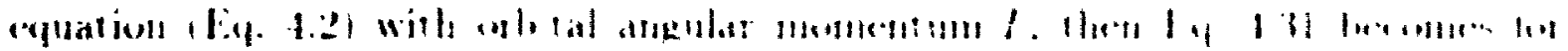
$-^{+}-\cdots$ produlion.

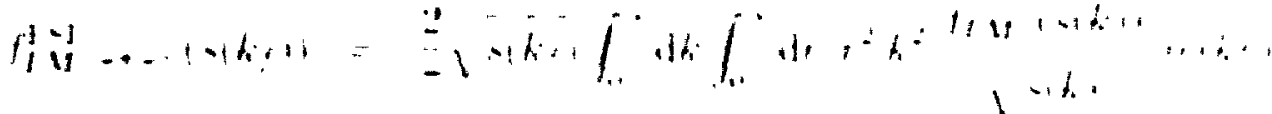

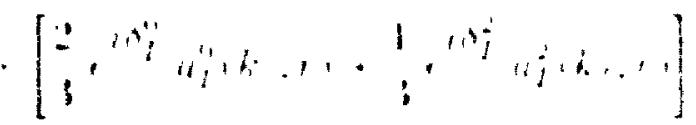

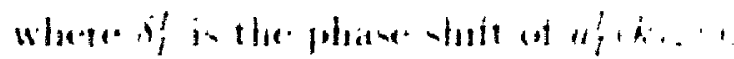

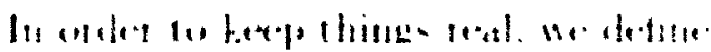

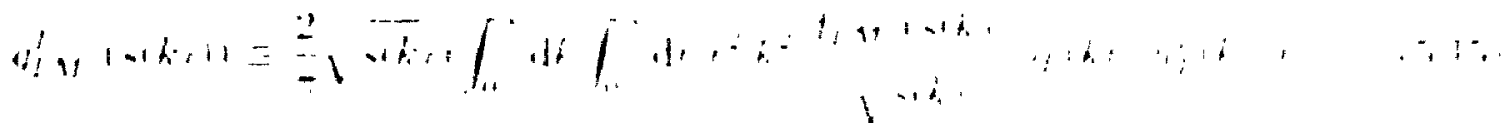

ninthens

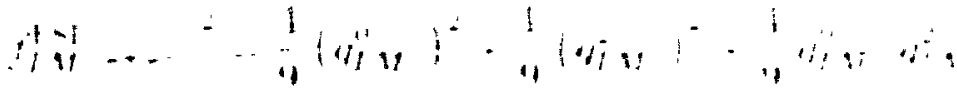

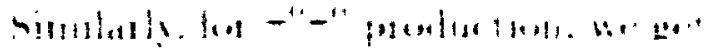

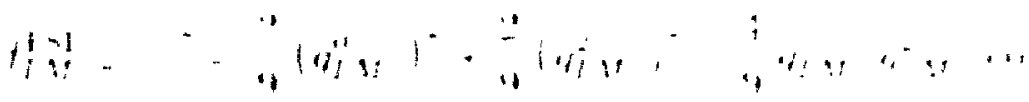

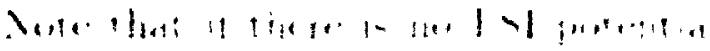

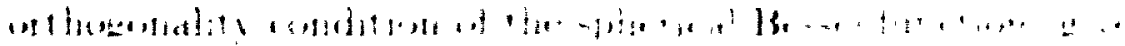



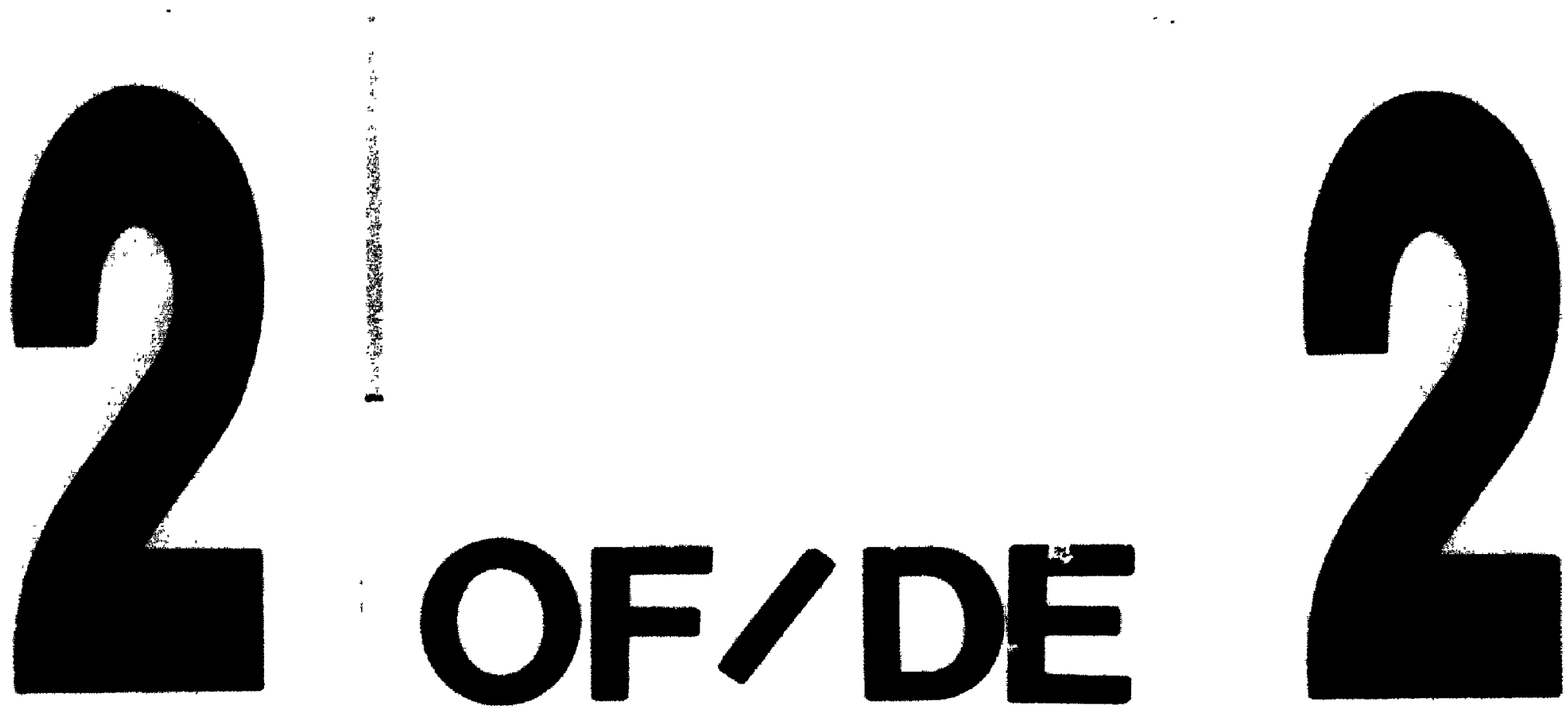

PA-1 3t/" 4" Photochaphic Macnocopy tancet wos woro Anstiso i2 EOUNALENT

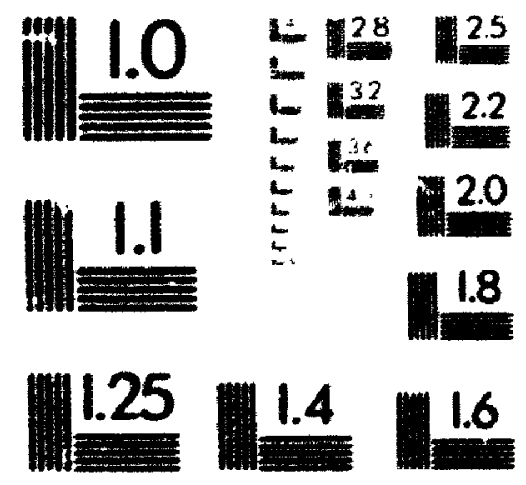

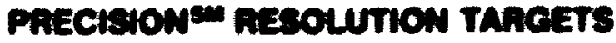




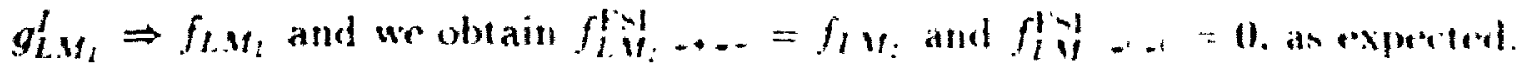

In this work. we only correct the $l=0$ partial wave. hecanes higher partial waves

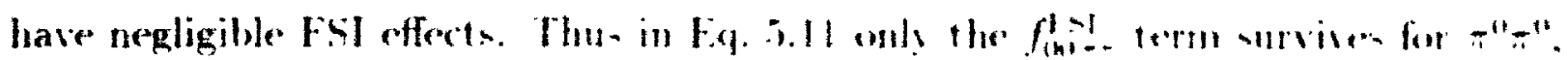
and for $\pi^{+}{ }^{-}$it is the only term that differs from the uncorrected vaiue.

\subsubsection{The Problem of Limited Polar Acceptance}

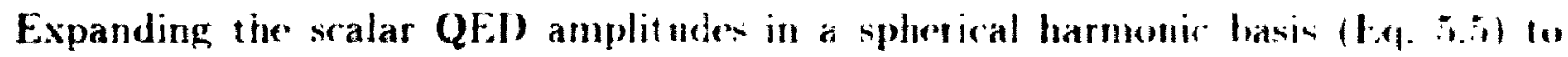
obtain cross-secticns for particular partial waves is esential to our calculationt, luit it requires that we be able fo integrate over the entire $1 \pi$ angular range whell caldulating the total eross-section. Infortmately experiment a domt measure data over the whole polar angle || $\cos \theta \mid \leq 0.6-0.5$ is typicall. we we ranmot easily compare and results with experiment.

Integrating to get the total uncorrected cross-section for a limited pelas acerle tance $\left(-\cos \theta_{\text {arer }} \leq \cos \theta \leq \cos \theta_{\text {as. }}\right)$ in not a problem: Fip. 5.2 in replared liy

$$
\begin{aligned}
& \sigma_{a r c}=\frac{\pi a^{2}}{4 m^{2}}\left[2 \cos \theta+x \sqrt{1-x}\left(\frac{r^{2}}{1-(1+r) n^{2} \theta_{a+}}+1\right)\right. \\
& \left.-r^{2}(2)-r \ln \left(\frac{1+\sqrt{1-x} \cos \theta_{a x}}{1-\sqrt{1-x}}\right)\right] \text {. }
\end{aligned}
$$

Howerer. the expression in terms of partial wave in afferted unerre diantixally: Eq. 5.6 becomes (where once again we drop, the explicit hedicity halesh)

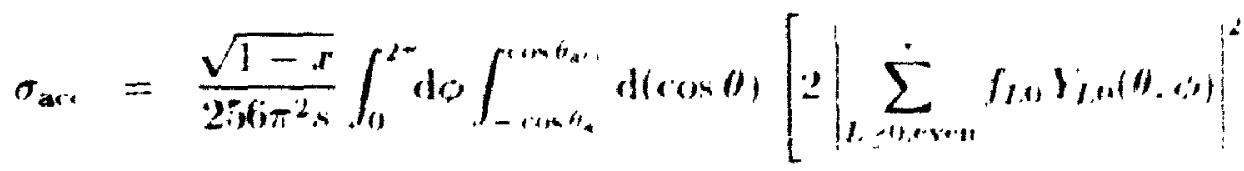

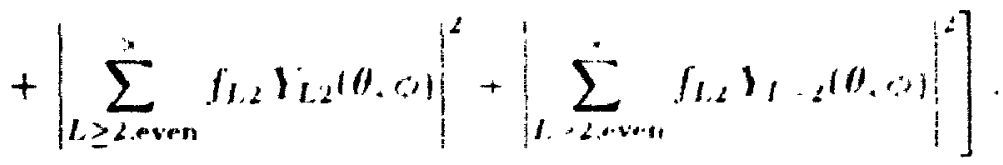

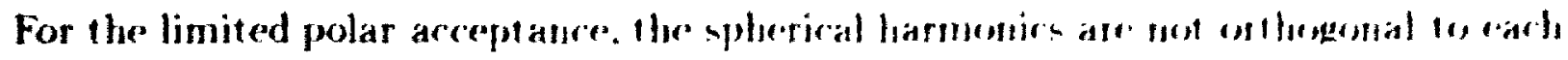


older. we earl magnitude rontain an infuite mumber of terms. As well. the contri-

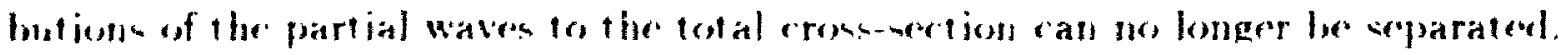

[herause the $l=0$ partial ware is experted to be the only one afferend significantly

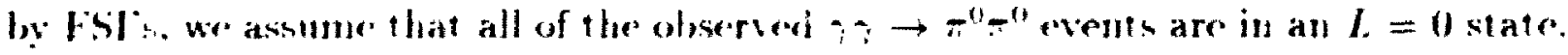
Simce that distribution is spherienlly symmetric. we can correct the data to fuli polat

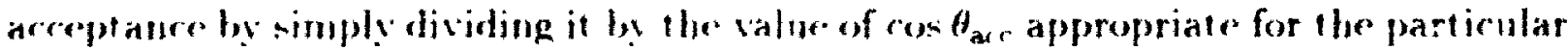
experiment

For in $_{7} \pi^{+} \pi^{-}$the total cross-section observed is a mixture of all partial waves: again, we assume that only the $I$. = O wave ic affected by IStis. Hefino

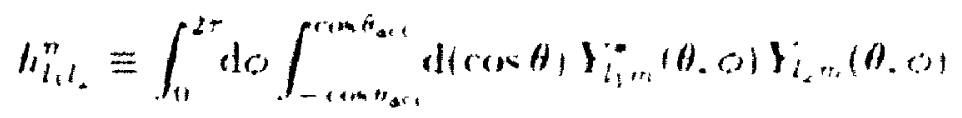

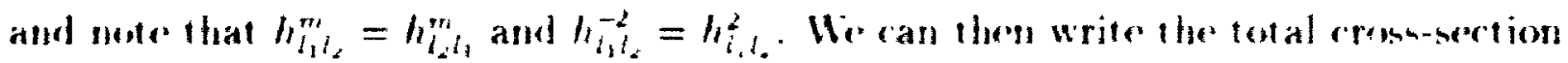
with only the $L=0$ partial wave corrected for fist as the lotal uncorrected crosssection. plas an infinite number of correction terms. each of which is the difference bet ween the corrected and uncorrected values of a term in lig. 5.19. Only those terms

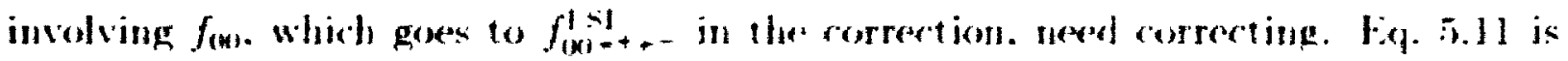
roplacod li.

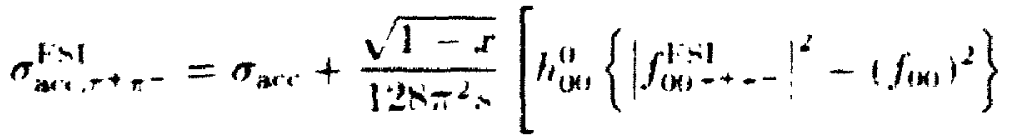

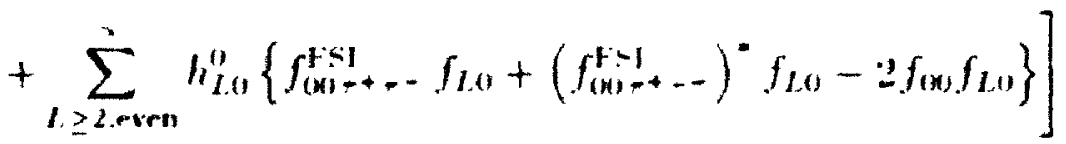

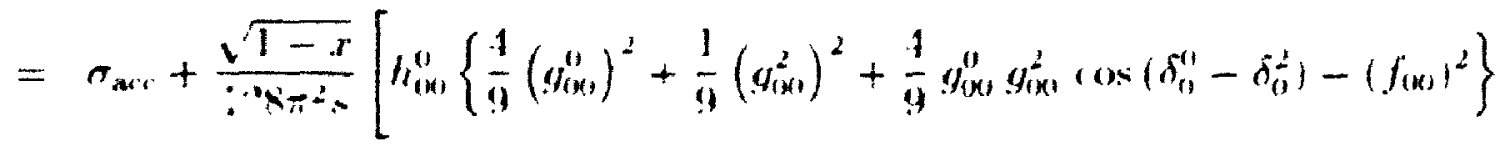

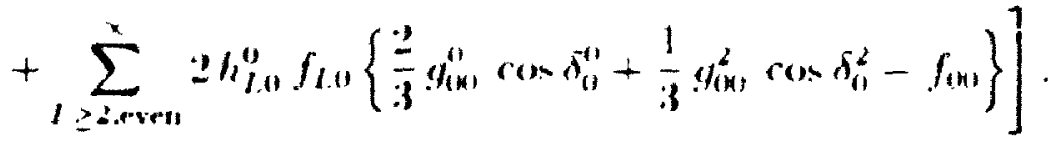

Wi. find that wo get excellent convergence over the energy gange we are interested in 
when we truncate the series after $l=$ t. Fortunatels, the three evperimem- whoms

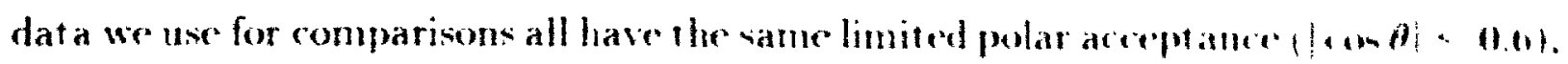
so we only have to correct our result for one value of cust at.

\subsubsection{Numerical Details}

Wr solved the radial Schrödinger equation ( Eq. 1.3 ) with the appropriate pentential

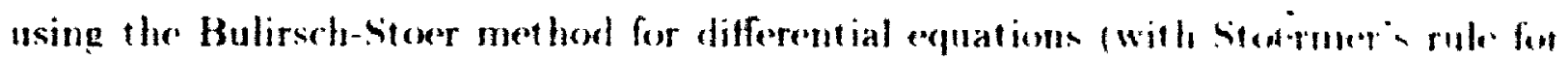

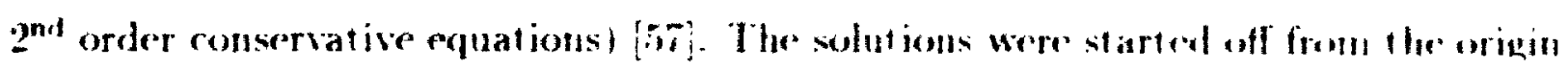
with the forms of $r$ times the spherical bessel functions of the same l. since the elferets of the potentials are insignificant at the origin. The amplitude and phese ahefe of

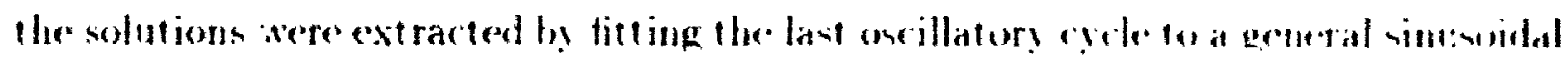

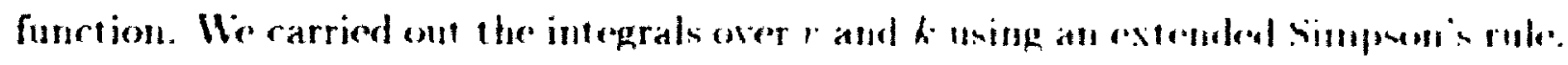

\subsection{Results}

We have calculated the effects of the FSt

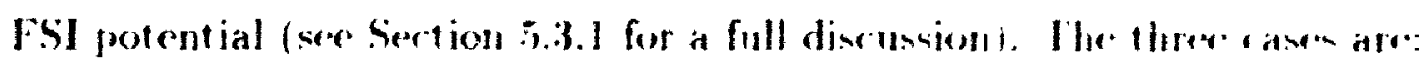

Case A. In this case we de not une 1 and a in the potentiale. Mie krem that the potentials will then fail to accurately predict the phase hiffe. In ardele to asoul

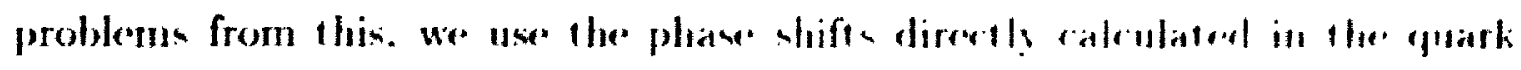
model in our expressions for ESI corrections. Fon mone information. we the detailed discussion on $y$ and $s$ on pagem ix Mhrough $x 11$.

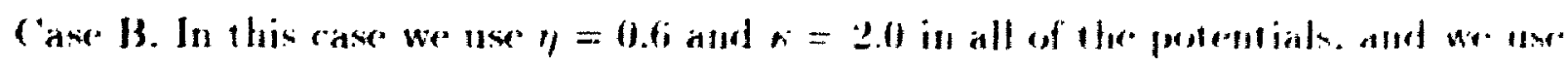
the resulting phase shifts in our expersions for Fil conted tions.

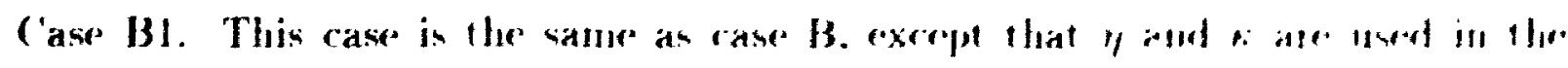
t-channel gluon exchange cont ribution to the pustentials bust met in the contei. 
bulon from stelannel gluon exchange.

In addition. wo bave three socondary casen. A'. B' and B1'. Fach of these in similar to the correspotiding unprimed rast. but dese not use the same phase shift in ont "xprexsions for Fil corrections. Instead. they use a simple (straight-line expression" for the phase shifts that attempts to describe the experimental data. We do this in order to get an idea of how much our results are affected by inacrurate phase shifts. If our results are poor because we have ignored a particular contribution to the potential (such as resonance effects). then using accurate phase shifts in the expresion for lisl rorrections would partly correct for this. Of course. we would still need to destermine the affect of the neglected contribution on the distortion of the wavefunction. and hener on the FSI effects.

Let us first examine the phase shift results. The $I=2$ phase shifts are shown in Figure 5.7. For the three cases A. B and B1. the curves shown are the phase shifts that come from solving the Schrödinger equation with the potent ials. The phase shift for ens" $A$ is peror. as expected: in the expressions for FSl corrections we will replare it with the curve calculated directly in the quark model. The plases shift for cases B and 131 are identical becanse they only differ in whether or not $y$ and $\mathrm{s}$ are indurled in the contribution to the potential from s-channel gluon exchange. which is not present for $l=2$. For the range of data shown. it is not clear which is better: the 13 and 131 phase shift s from the Schrödinger equation. or that from the quart model. However. data at somentat higher energies favours tho guark model rurve. The remaining curve shown is just a straight-line which we need to describe the data for the primed aste.

\footnotetext{
The st raghe -lue expressens we use to deserite the phase shift are

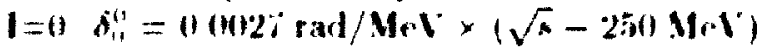
$I=2 \quad A_{i}=-000062 \mathrm{rad} / \mathrm{M} C \mathrm{~V} \times(\sqrt{x}-250 \mathrm{MeV})$
} 


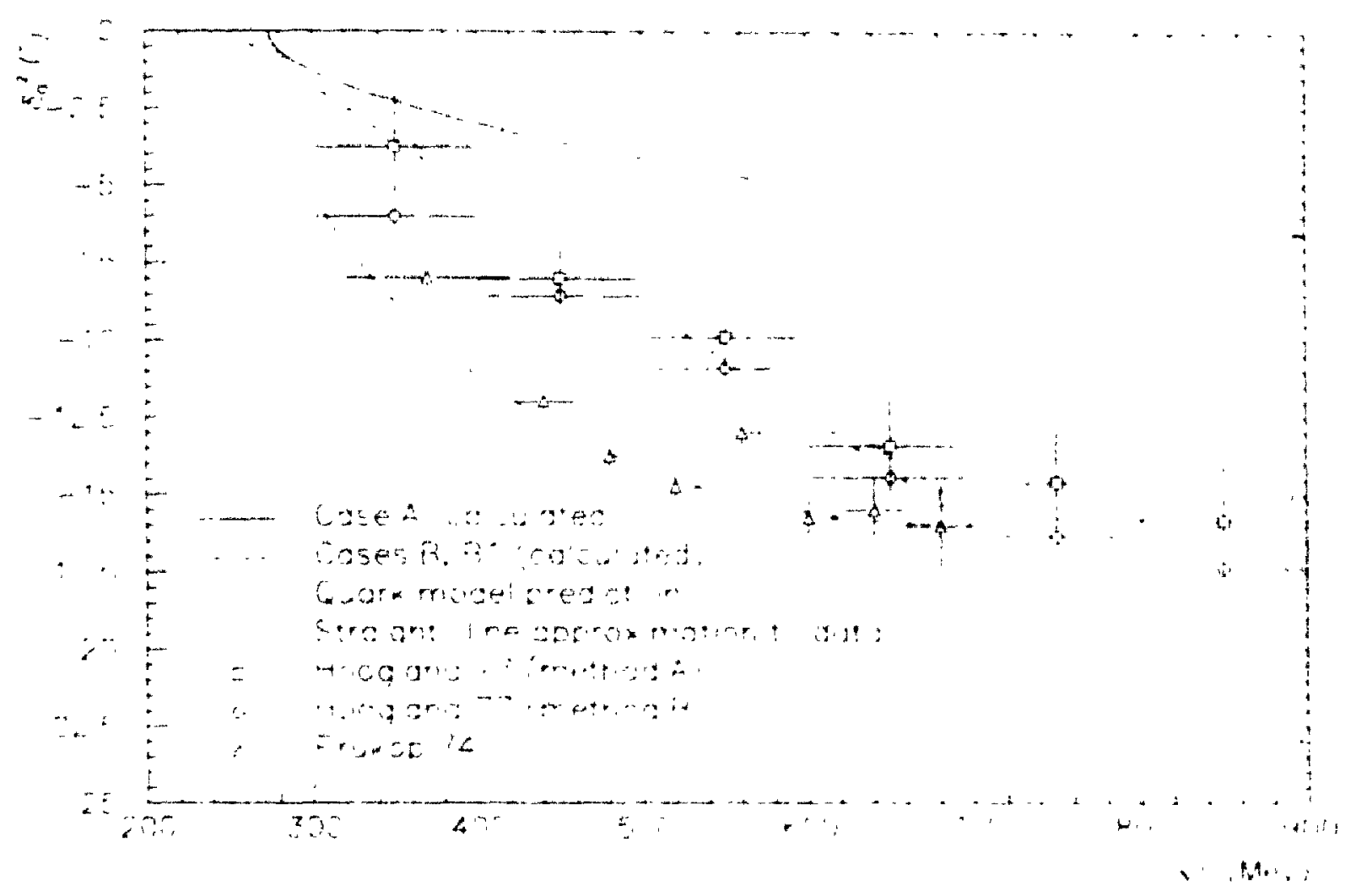

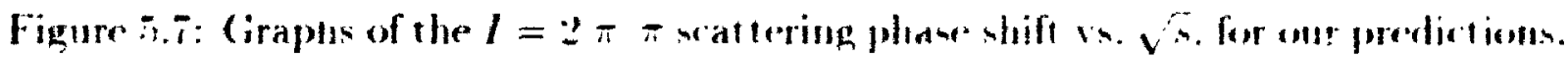
the quark nodel prediction. and experimental data. The data is from Ileoggland of

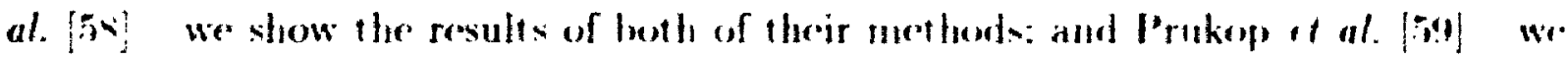
show the results of their first fit. For additional commonts sere Figure 5.1 .

The $I=0$ phase shifts are shewn in Figure is. Note the rather unfortunats.

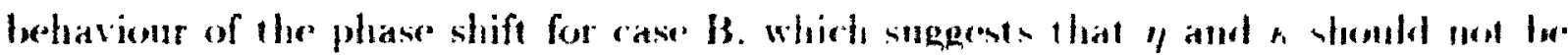
used in the s-chanel gluon exchange comt ribution to the penential. Simes there in ne

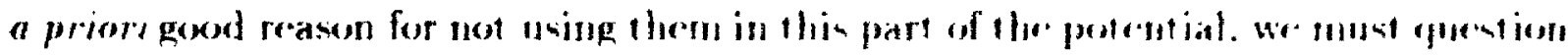

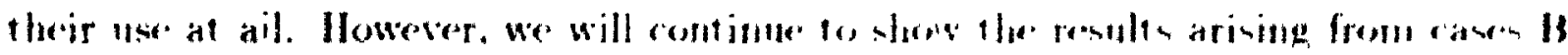
and B1. Again. the phase shift for rase $A$ is pers. and fon Fil correction we will replace it with the curve arising directly from the guatk medel. Neate that thin time

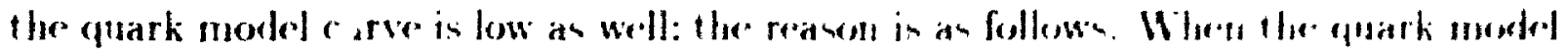
predictions for the $I=0$ phase shifte were calculated fil there comtributione wese considered: t-channel gluon exchange. s-rhannel gluon exchange. atud contritontions 


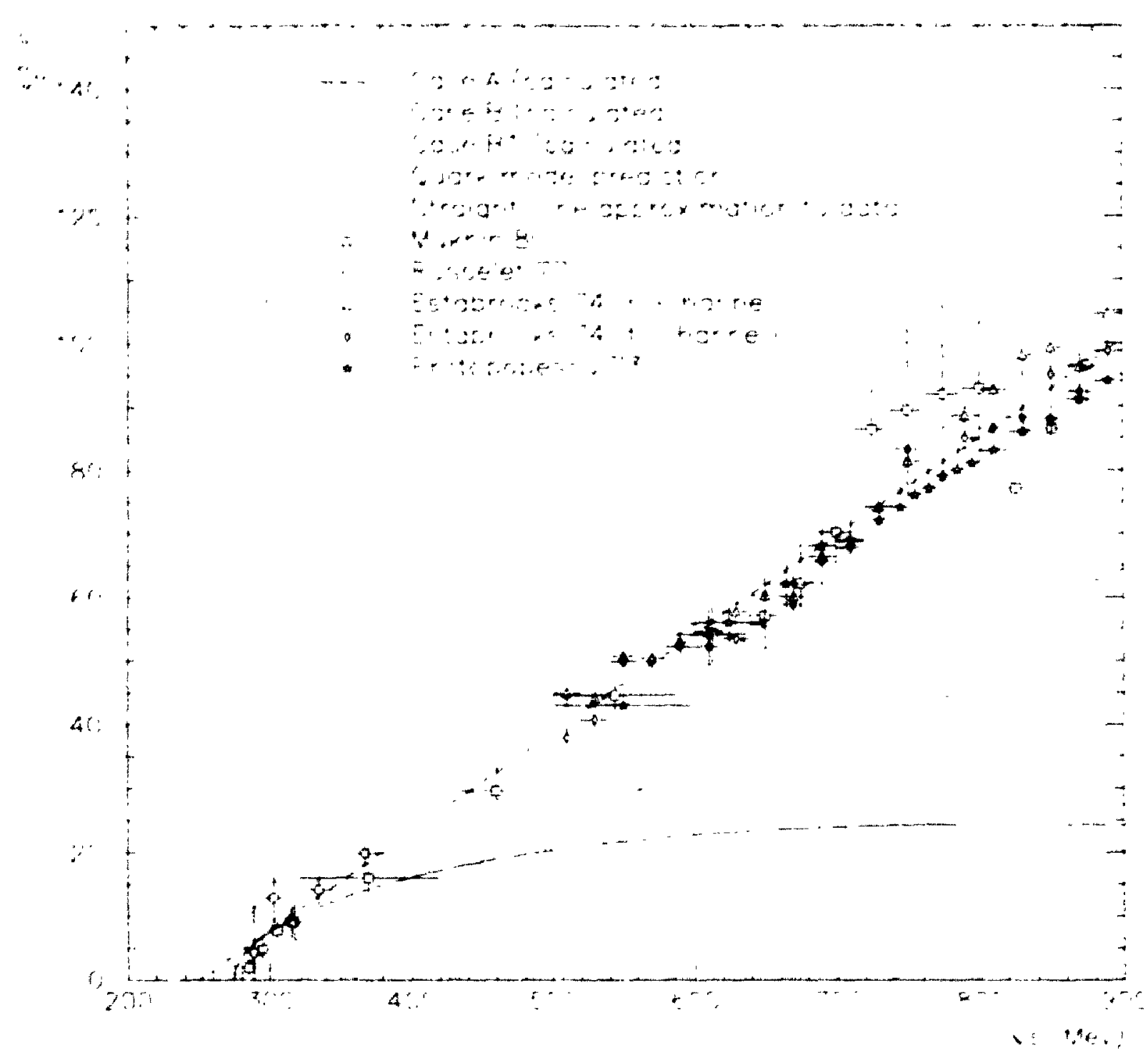

Figure is: (iraphs of the $l=0 \pi \pi$ satterine phase shift $\cdots$. 5 for our predictions. the quark model prediction. and experimental data. The data is from Mukhin at al. [60]: Renselet of al. [61] the horizontal bar only approximate their bins and their dat it is actually for $\delta_{10}^{\prime \prime}-\delta_{1}^{\prime}$ we have used the $\delta_{3}^{\prime}$ dat a extracted from it by li et al. [i]]: Estabrooks and Martin [62] wo show the resuli of both their s- and 1 -channe] fits: and Protopopesce it al. [6.3] we show the resu]t of their case 1 . For additional comments sere Figure \$\$.1.

from interactions with the $f_{0}(980)$ and $f_{0}(1300)$ resonances. These cont ributions were fit to the data. but because's are not including resonance effects in this work. we only include the cont ribut ions from the s- and t-channel gluon exchange in our quark 


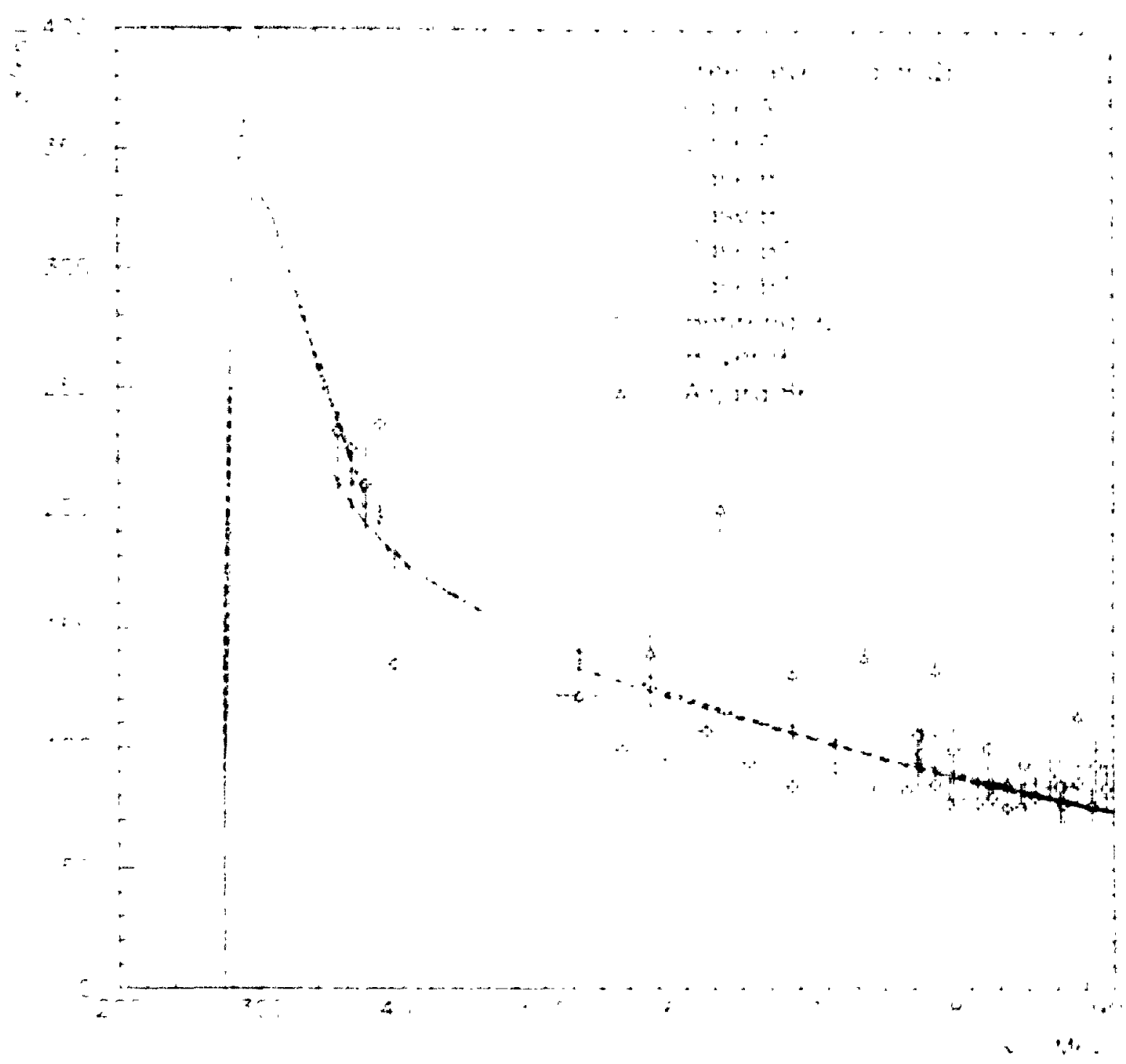

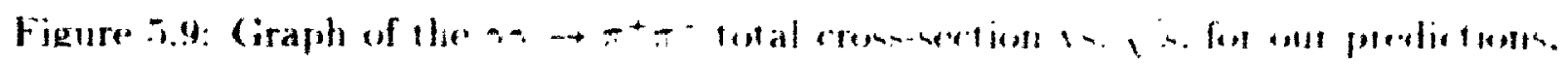

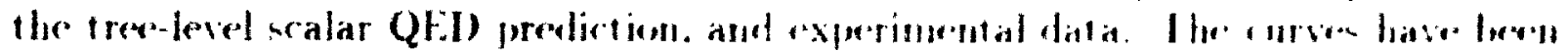

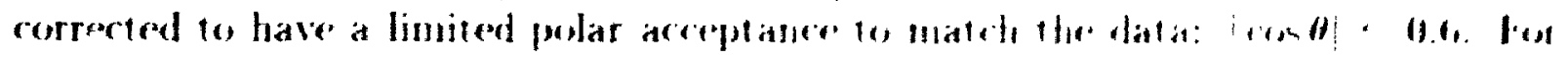

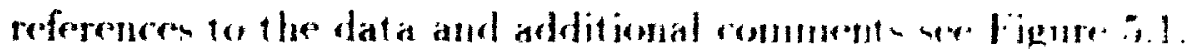

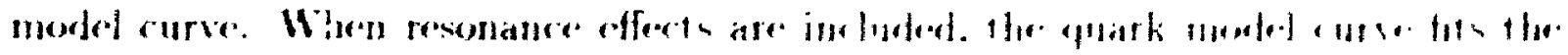

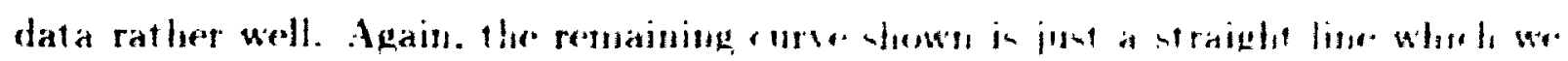
have uned to dascribe the data for the primed, ...e.

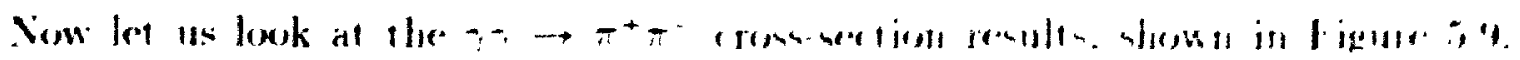

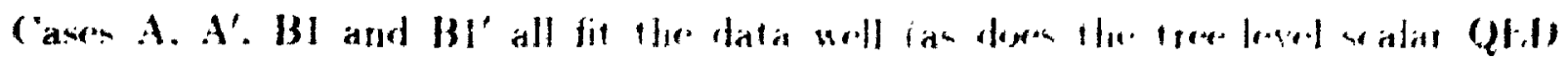




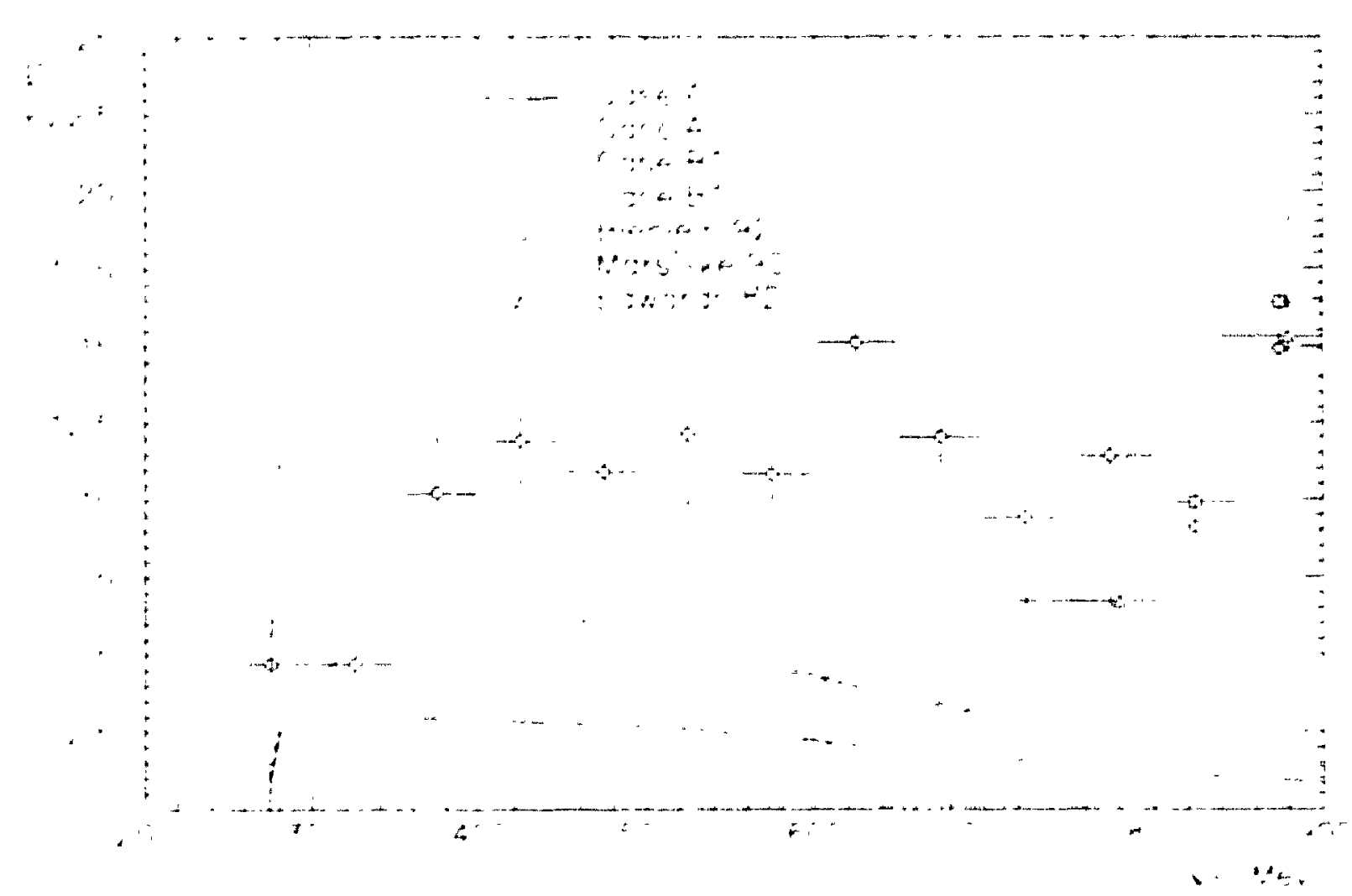

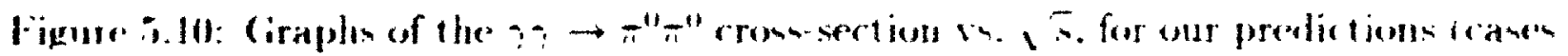

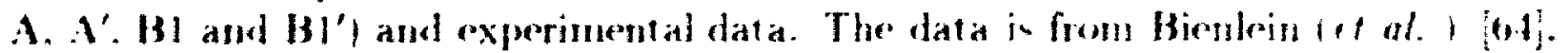
Marviske et al [6i] and Edwards of al. [6fi]. The data has heesh corrected we full polar acceplance: $|\cos \theta| \leq 1$.0. For additional comment we Figure i.l.

predictiont. The effert of using the quark model phase bift are not significant for

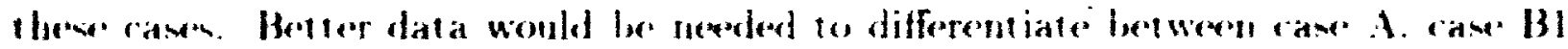
and the tree-level watar QED prediction. (ases $B$ and $B$ are dinfavoured he the data. as might be expected from the peor performance of case $B$ in predieting the phase

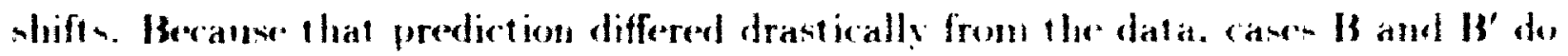
differ appreciahls in their predictions of the cross-section.

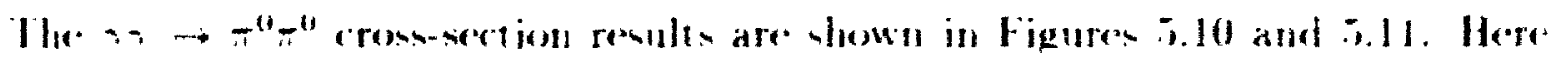

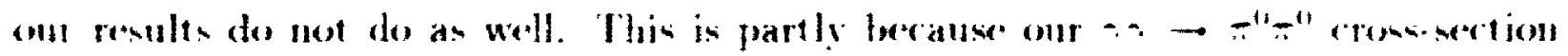
is wholly dese to Fsle while in the $a \rightarrow \pi^{+} \pi^{-}$case the FSl effect are corrections to a more signiticant corve. Thi als, means that the primed and unprimed casen 


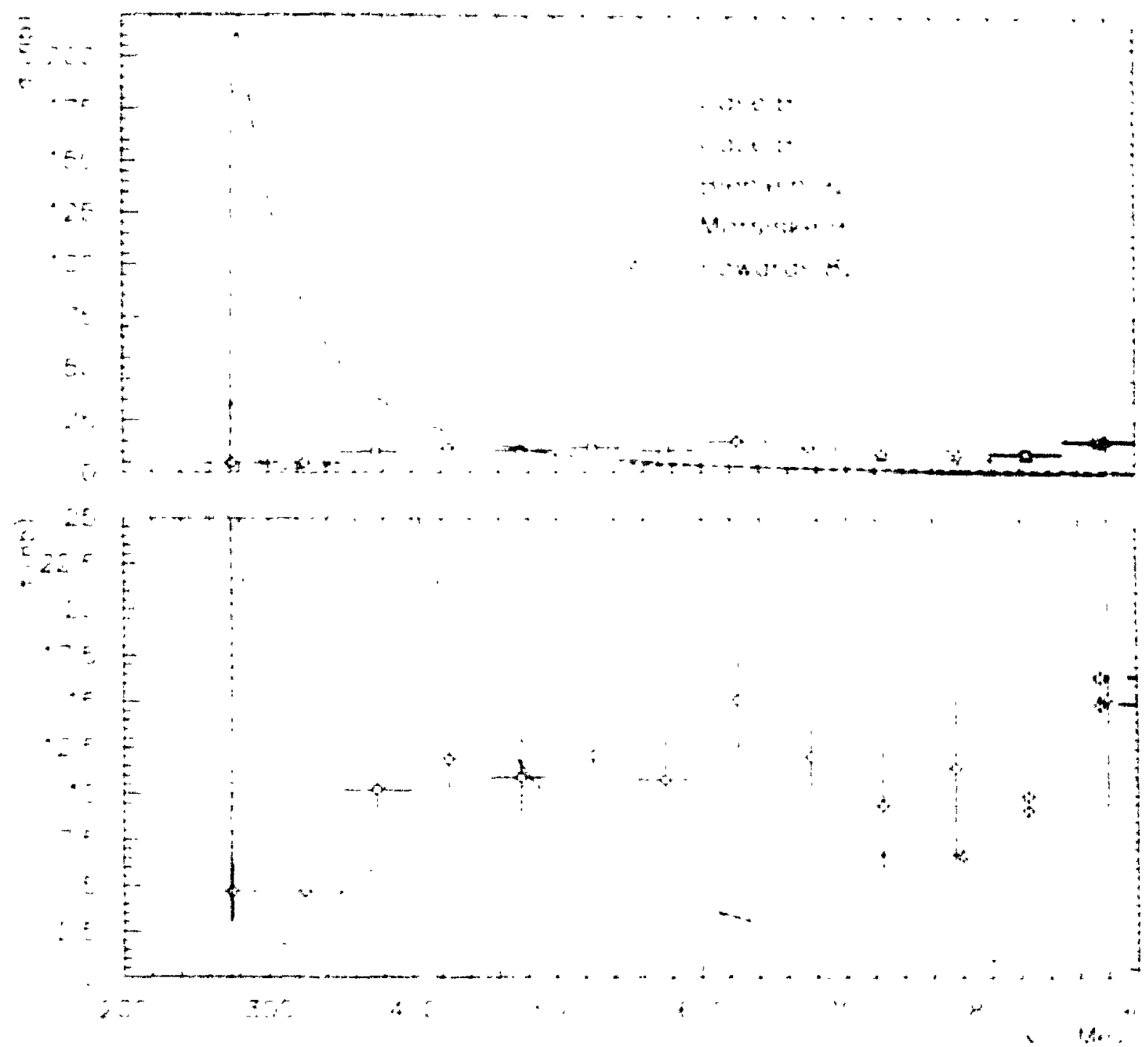

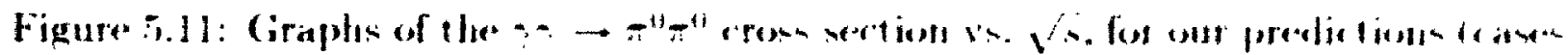

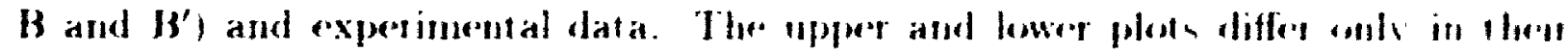

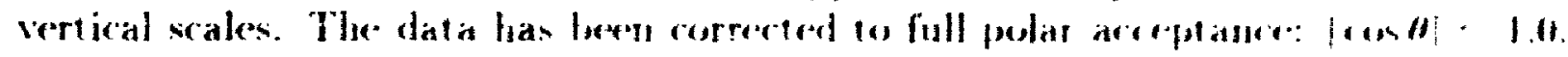
For references to the data exe Figure s.10. For additional comment we Figure il.

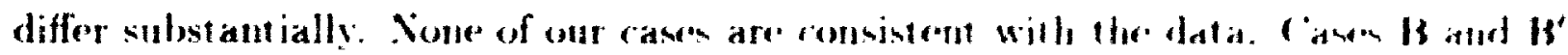
de particularly badly an expected from and previous results. (of the athere tase $A$ '

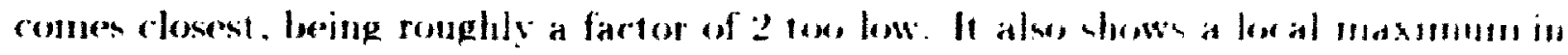

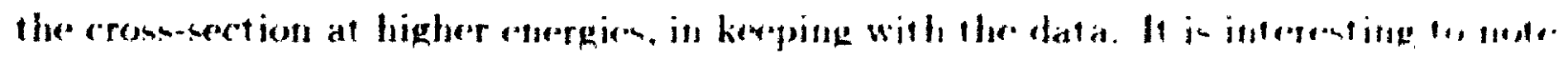

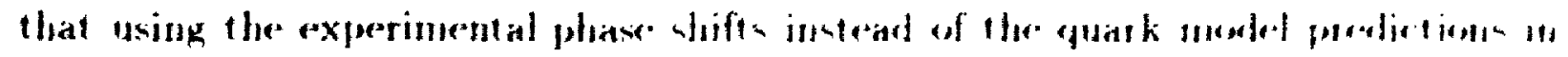


our rexpressions for the FSI rorrertions greatly improves curve $A$. Since the differene in these phase shifts is due to resonanere effects. we tend to believe that including the effert of resonances in the wavefunction distortion as well wonld improve the curve still further.

We have demonst rated that the quark model can predict the $\eta \rightarrow^{+}-^{-}$crosssection in agreenent with experimental data when a sufticiently detaled calculation is performed. However, with the present potentials it is unable to accurately predict the $7 \rightarrow \rightarrow \pi^{0} \pi^{0}$ coss-wetion. We lind that using Weinstein and Isgur's $\eta$ and $x$ factors in the potentials is not helpful when calculating FSI ffects: it is better to use the expressions for the phase shifts calculated direrly in the quark model in the expressions for the FSI corrections. We believe that the next logical step in this progeram of nork is 10 include potentials allowing the $I=0 \pi$ state 16 convert : the $f_{1}(3 \times())$ and $f_{0}(1300)$ resonances (and back). Thi would mean solving a coupledchanuel Schrölinger equat ion in the $1=0$ channel. 


\section{Chapter 6}

\section{Conclusions}

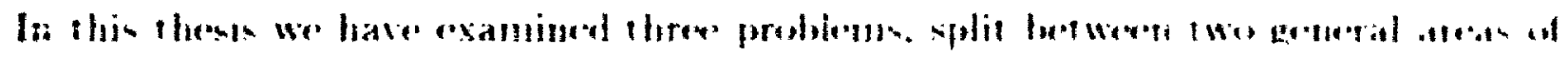
the quark model.

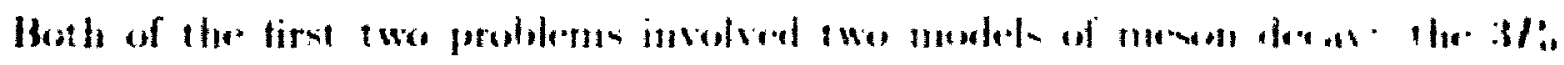

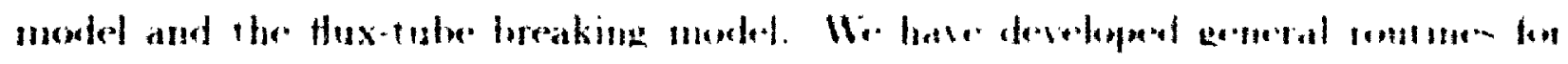

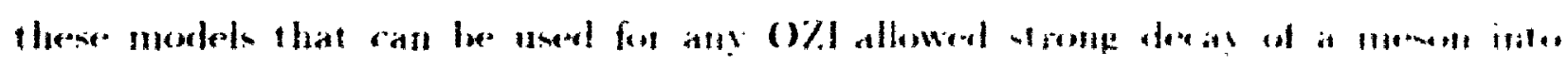

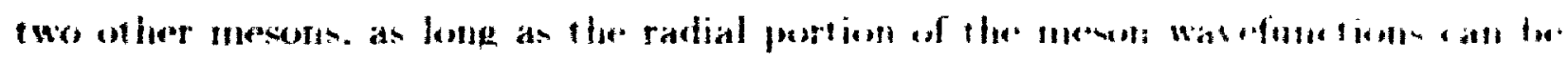

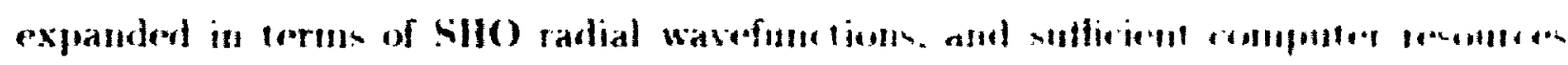

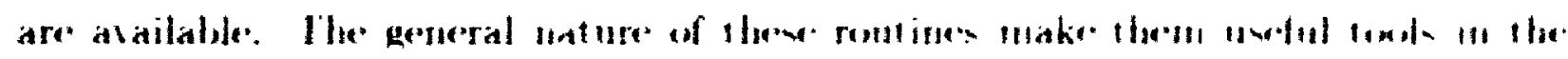
study of meenon phernomenology:

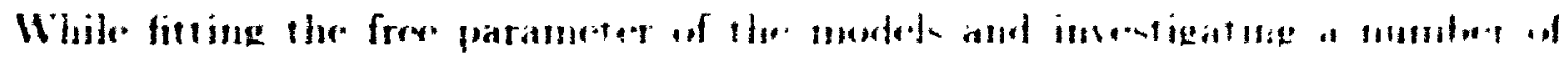

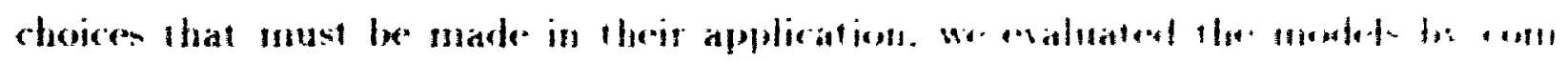

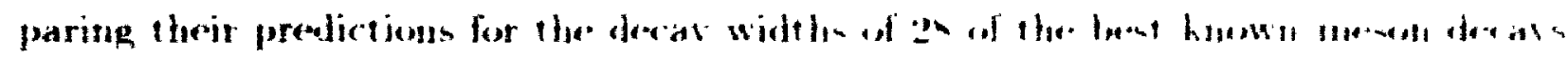

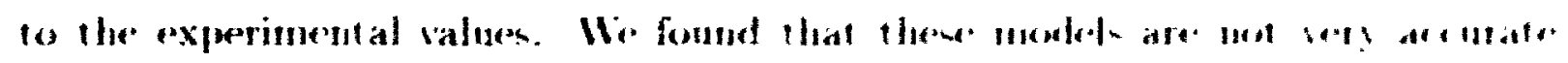

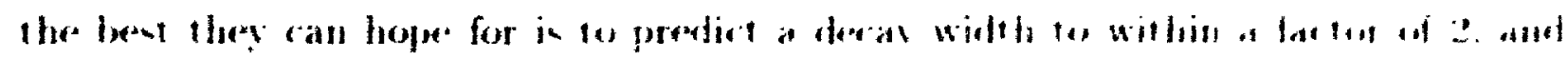

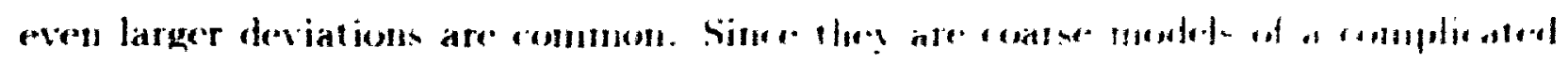

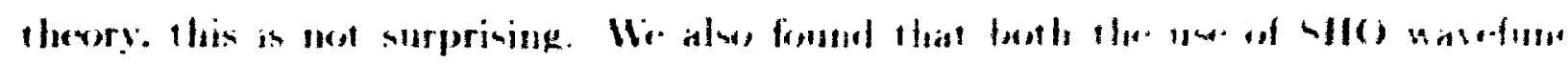

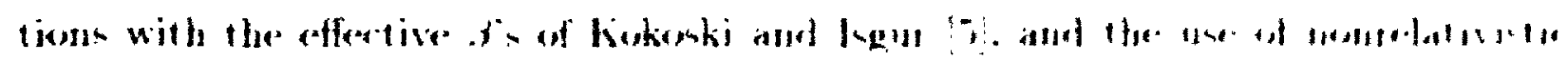


phase space/normalization give less aceurate results than can be obtained with other dioiren.

The first problem that we investigated was the identity of the fal 22201 . Although

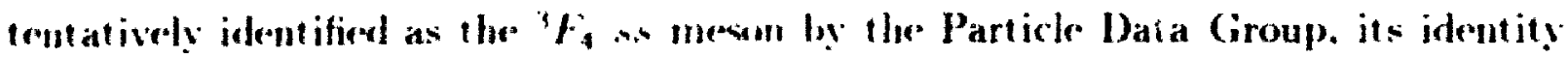
has Inen uncertain since its discovery in 1983. We carried out detailed calculations of the decays of the "F and " $F$ se mesons in order to see if a meson identification for the $f_{4}(2200)$ is tenable. Despite the uncertainties of the decay models. we found that the fal2020), with a measured decay width of approximately 30 Mel: cantot be the " $F$.... whese width we expect to be 2400 Met. He expect the width of the "F. weron to be 2140 Mel and $\leq 600$ Mel: but the uncertainty of the models

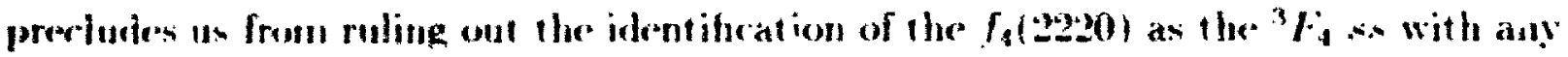
surety. Mi- do feed it is unlikely however. and propose the followisg explanation: that the hroad sate wen in hadron heam experiments is the " $/ 4 . . m$ meson. and the narrow state seren in $J / 4$ radiative decay is a glueball. Further experimental data is needed

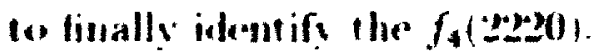

The second problent that we investigated was the determination of the mixing angle bet wern the $h_{1}(1250)$ and $h_{1}(1,100)$ mesoms. This was done ty comparing predietions of the meon deray models for tive partial decay width and wor ration of D) wo stuplitules with experimental data. We foumd the mixing angle $\theta_{h}$ to be approximately 1.t. If the mixing is due only to the spin-orbit interactions already included in the quark momel of (iodfres and kaur. then this implies that the responsible term in the Haniltonian. $H_{3,-}^{\text {in }}$ has a value far larger than calculated. However. because of the delicate atreellation that loads to the small calculated value. we are unable to draw any conclusions about whether the mixing is largely dus to another mechansin.

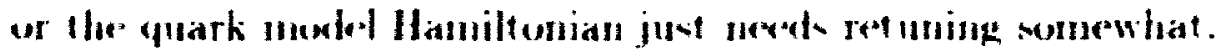

The third problem that ne insentigated was the effect of the st rong final state

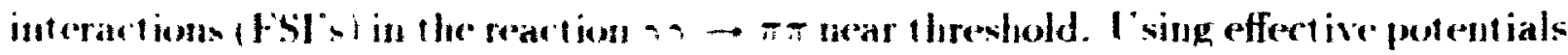




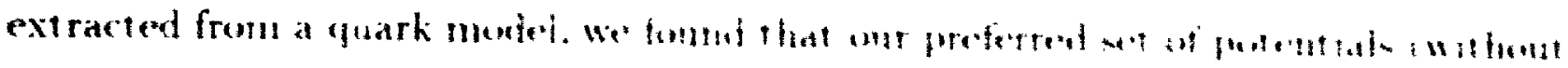

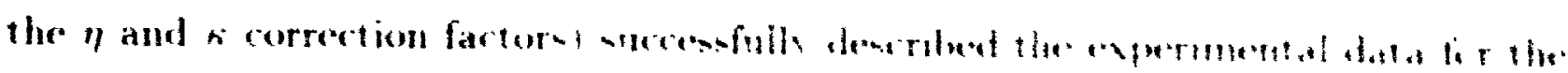

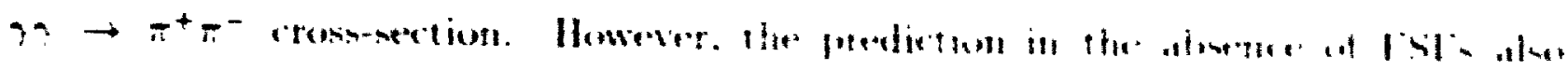

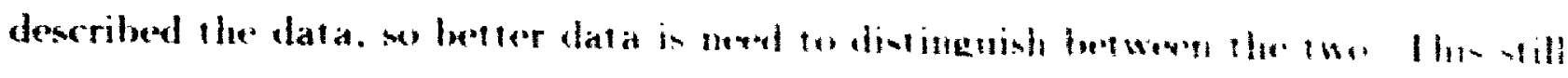

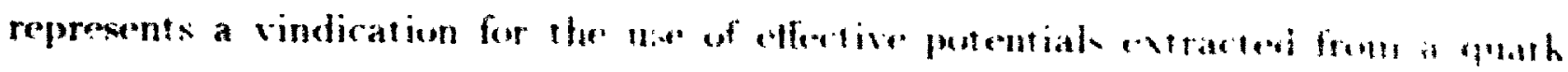

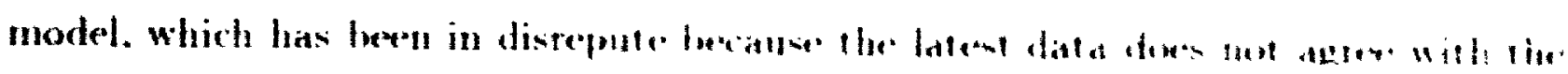

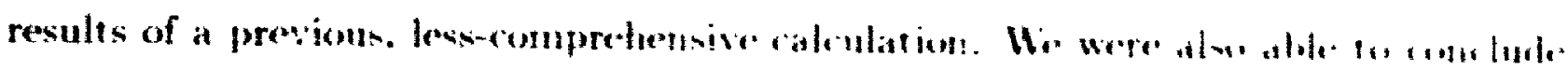

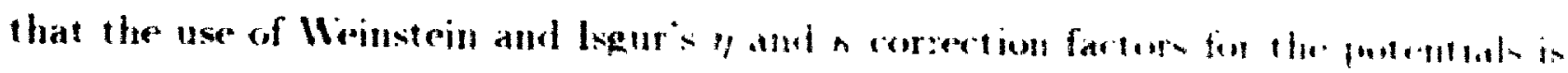
inappropriate for the calculation of Fst e.tferts.

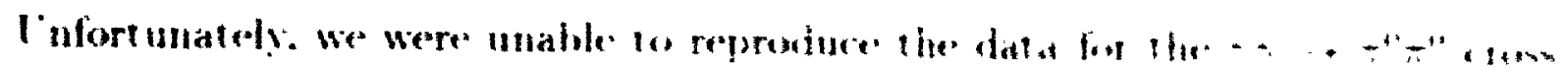

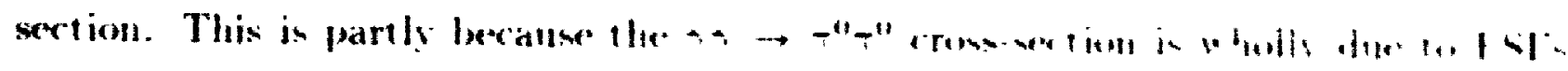

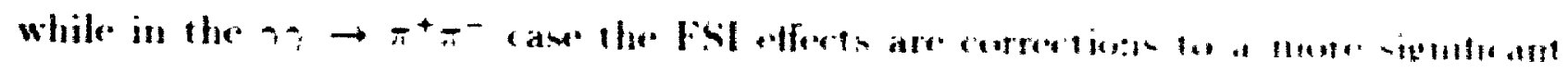

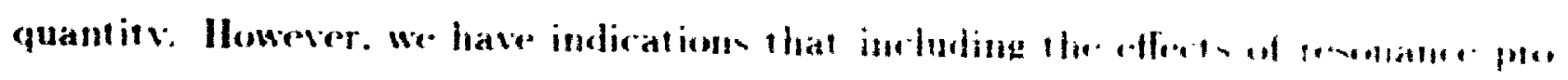

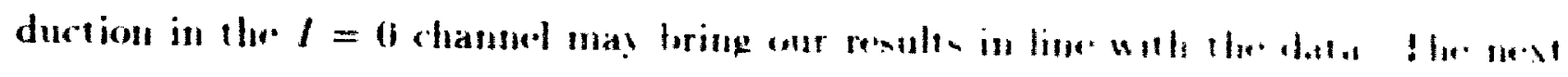

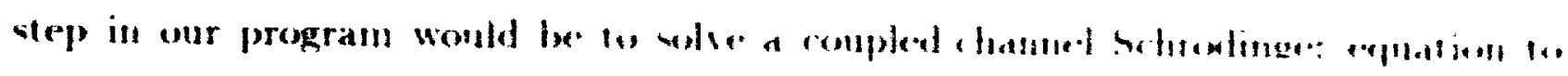

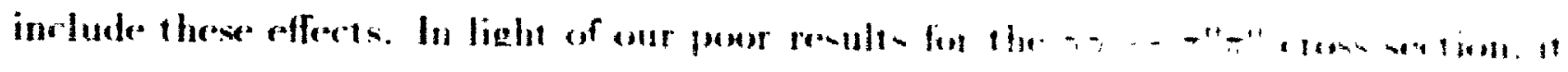

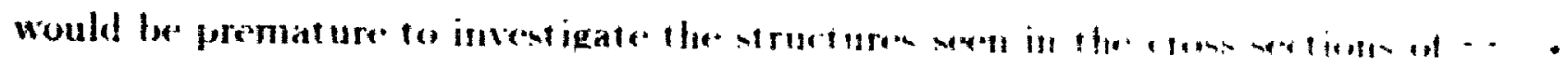
two rector mesols at this time. 


\section{Appendix A}

\section{Some Tools of Particle Physics}

In this appendix we int roduce some of the terms and tools of particle physics. to help specialists in other fields in understanding the work of this thesis. For an excellent int rodurtion to the field. see (iriffiths $[67]$.

\section{A.1 Cross-section and Width}

In most particle physics experiments two particles are collided and the outgoing particles are collected and identified. Due to the interactions between the particles, they may be scattered in different directions. and/or they may be transformed into other partirles. Thr probability of a particular reaction occurring is related to the crosssection $\sigma$ which is a function of the total energy of the particles and is measured in units of barns, or $b\left(1 \mathrm{~b}=10^{-28} \mathrm{~m}^{2}\right)$.

A particle will decay event ually if there is an interaction that permits it to be transformed into other particles with less total mass. The average lifetime of the particle is denoted by $\tau$ : by Heisenberg's uncertainty principle. this finite lifetime leads to an uncertainty in the mass of the particle. In a collision of two particles. the production of a short-lived particle in the intermediate state (which then decays 
to give the final state particles) shows up in the cross-section plot as a resonance described by a Breit-Wigner curve with width $\Gamma=1 / \tau$ (see for example Figure 3.T).

For a particle that can decay into two or more different final states we can defime its branching ratios, which are the probabilities that the particle will decay into the various final states. From these we can define the partial width for a particular decay mode as the product of the branching ratio for that mode, and the tot al width. The sum of all the partial widths gives the total width, which is what is used to get the particle's average lifetime. We may sometimes refer to a partial width as the width for a particular decay mode.

Similarly for the cross-section for a particular initial state. there are the crosssections going to specific final states. which sum to give the tot al cross section. Note that in a plot of the cross-section to a specific final state, a resonance in the intermediate state still produces a Breit-Wigner curve with a width equal to the total width for that resonance. (Even though we are only looking at a particular decay mode. the lifetime of the resonance is still that determined by all of the possilble decay mordes.)

\section{A.2 Feynman Diagrams}

In a perturbation theory such as that normally used to ralculate quantities in Qlil). each order of perturbation is represented by Feyman diagramm, such as those shown in Figure 5.2 for $\eta \rightarrow \pi^{+} \pi^{-}$in scalar QWD. In a Feymman diagram the particless are represented by lines, and basic interactions by vertices where a number of lines meet. Since for QED each vertex corresponds to another factor of $\sqrt{1}$ in the Feymman amplitude' the order of the perturbation is given by the mumiser of vertices in the diagram.

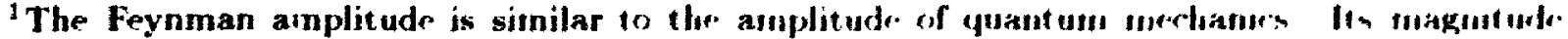

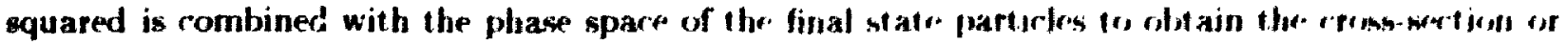
width.
} 
A tree-level diagram is one in which there is only one path of lines connecting any two vertices (i.e. there are no loops). For $\mathrm{QED}$ at least. if a tree-level diagram exists for a particular process then it is the lowest order diagram.

\section{A.3 Clebsch-Gordan Coefficients and the Wigner nj Symbols}

All angular momentum state, be it spin. orbital angular moment um. or a vector sum of some combination of these, is represented (in an $S(1(2)$ algebra) by the usual quantum numbers $|j m\rangle$ where the magnitude of the total angular momentum is $\sqrt{j(j+1)}$ and the z-component is $m$. To combine two angular momentum states we use the ClebschGordan coefficients. defined by

$$
\left|j_{1} m_{1}\right\rangle\left|j_{2} m_{2}\right\rangle \equiv \sum_{j=\left|J_{1}-J_{2}\right|}^{J_{1}+j_{2}}\left\langle j_{1} m_{1, j_{2}} m_{2} \mid J M\right\rangle|J M\rangle
$$

whrere $\left\langle j_{1} m_{1} j_{2} m_{2} \mid J M\right\rangle$ is the ('lebsch-Ciordan coefficient and $M=m_{1}+m_{2}$.

The Wigner $3 j$ symbol is related to the Clebsch-Gordan coefficient by

$$
\left(\begin{array}{ccc}
i_{1} & j_{2} & . J \\
m_{1} & m_{2} & -M
\end{array}\right) \equiv \frac{(-1)^{\mu_{1}-J_{2}+M}}{\sqrt{2 J+1}}\left\langle j_{1} m_{1} j_{2} m_{2} \mid J M\right\rangle
$$

The Wigner $6 j$ symbol

$$
\left\{\begin{array}{lll}
j_{1} & j_{2} & j_{3} \\
J_{1} & J_{2} & j_{3}
\end{array}\right\}
$$


is use d to combine three angular moment um states. and the Wigmer 9, simbol

$$
\left\{\begin{array}{ccc}
j_{1} & j_{2} & J_{12} \\
j_{3} & j_{4} & J_{34} \\
J_{13} & J_{24} & J^{\prime}
\end{array}\right\}
$$

is used to combine four angular momentum states. We will not define these here. though the $9 j$ symbol is defined in Appendix D.:3. For nore information, ser for example Reference [34].

\section{A.4 Conservation Laws and Invariance Principles}

Because of symmetries in the theories of the interactions. the interactions themselves obey a number of conservation laws and invariance principles (ser for cxample Re.fer. ence [54]). We will only discuss the ones of interest here.

There is the usual conservation of energy, momentum, angular momentum and charge.

If one thinks of an antiparticle as being - 1 particles, then the munbers of heptons and guarks are separately conserved as well this is enforced fw the allowed interactson vertices (e.g. if an ${ }^{+}{ }^{-}$pair is created. the change in leptom mumber is $(-1)+(+1)=0)$.

Because the strong interartion is independen t of the type of guark involved. and the $u$ and $d$ quarks have very similar masses. we cant think of thesn as different projections of the same state which we represent by the angular moment um like quantity called isospin (where $u=\left|\frac{1}{2} \frac{1}{2}\right\rangle$ and $d=\left|\frac{1}{2}-\frac{1}{2}\right\rangle$ ). Isospin is conserved in the. strong interaction to the extent that the masses of the $u$ and d quarks arr degene.rate.

The spin-statistics theoren govern how systems of identical particlee bediave. when two of the particles are exchanged (boson wavefunctions ate unaffected. fermichn 
wavefunctions pick up a factor of -1 ). It is important that the wavefunctions we construct obey these symmetries. In the context of the strong interactions. members of the same isospin multiplet also obey the spin-statistics theorem, even though they are not strirtly identical.

The parity of a system indicates how the wavefunction behaves under the space inversion $\vec{r} \rightarrow-\vec{r}$ : the wavefunction is either unchanged $(\mathcal{P}=+1)$ or picks up a factor of $-1(\mathcal{F}=-1)$. The total parity of a system is conserved in the electromagnetic and strong interactions. but not the weak interaction.

linder thr charge conjugation operator. particles are changed to their antiparticles (and vice versa). Systems which are eigenstates of $\mathcal{C}$ (such as $\pi^{\circ}$. or the $\pi^{+} \pi^{-}$system) may have $\mathcal{C}=+1$ or -1 . (harge conjugation is also conserved in the elect romagnetic and strong interactions, but not the weak interaction. Charge conjugation can be extended by combining it with isospin to get G-parity. which is conserved in the st rong interaction only.

\section{A.5 Relative Coordinates}

The wavefunction of two particles in position-space depends on the coordinates of the two particles, $\vec{r}_{1}$ and $\vec{r}_{2}$. When solving the Schrödinger equation it is convenient to separaic it into equations involving the centre of mass (CM) coordinate of the particles. $R$, and their relative coordinate $r$;

$$
\begin{aligned}
& \vec{R}=\frac{m_{1} \vec{r}_{1}+m_{2} \vec{r}_{2}}{m_{1}+m_{2}} \\
& \vec{r}=\vec{r}_{1}-\vec{r}_{2}
\end{aligned} \Leftrightarrow \begin{aligned}
& \overrightarrow{r_{1}}=\vec{R}+\frac{m_{2}}{m_{1}+m_{2}} \vec{r} . \\
& \vec{r}_{2}=\vec{R}-\frac{m_{1}}{m_{1}+m_{2}} \vec{r}
\end{aligned} .
$$


The conjugate momenta $\vec{p}_{1}$ and $\vec{p}_{2}$. and $\vec{p}$ and $\vec{p}$. are relatenl hy:

$$
\begin{aligned}
& \vec{p}=\vec{p}_{1}+\vec{p}_{2} \\
& \vec{p}=\frac{m_{2} \vec{p}_{1}-m_{1} \vec{p}_{2}}{m_{1}+m_{2}} \Longleftrightarrow \overrightarrow{p_{1}}=\frac{m_{1}}{m_{1}+m_{2}} \vec{p}+\vec{p} \\
& \vec{p}_{2}=\frac{m_{2}}{m_{1}+m_{2}} \vec{p}-\vec{p}
\end{aligned}
$$

This is a nonrelativistic construction: $p$ remains unchanged when both particla are given the same velocity boost only for nonrelativistic velocitien.

The CM frame refers to the inertial reference frame in which $\vec{P}=0:$ i.c. in which the centre of mass of the systen is motionless.

\section{A.6 The OZI Rule}

Okubo. Zweig and lizuka (OZI) noticed that Q('D) interactions in which all of the" initial quarks are annihilated. and are not present in the fiual state. are suppresient. This is thought to be because for interactions of this lype. asymplotic frodemu mealss that the strong coupling constant $a_{s}$ (which enters the cross-ention as at lanst ons) becomes small since all of the energy of the initial state particles must be inducleal in the moment um transferred through the gluons (ser for exanule Reforence [fix]).

\section{A.7 Meson Mixings}

Particles with the same "good" quantum numbers (i.e. a complete set of communt ing observables) "mix" if there exists an interaction through which ond particlu ean be transformed into the other. and vice versa. For ratumple. the anange mestum

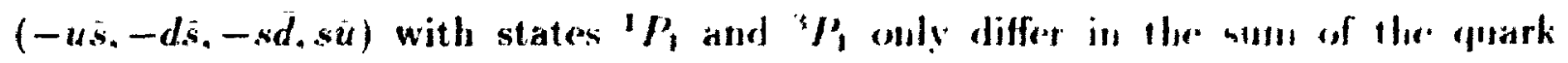
spins, and can mix via the spin-orbit interaction (and possilly otleres to prendere. the physical states $h_{1}(1270)$ and $h_{1}(1400)$. Another rample is fonnd in the llavoust 
wavefunctions: the $u \dot{u}, d d$ and $s \bar{s}$ are all flavourless (since the flavour of the $u$ cancels that of the $u$ ) and so there is nothing to prevent the flavour states in a ${ }^{\prime} S_{0}$ spin/space state (for example) from mixing by an annihilation interaction to produre the physical states $\pi^{\theta} . \eta$ and $\eta^{\prime}$.

By mix, we mean that the basis of quantum number states (e.g. $h^{\prime}\left({ }^{1} P_{1}\right)$ and $\left.K\left({ }^{3} P_{1}\right)\right)$ differs from the basis of the physical states (e.g. $K_{1}(1270)$ and $\left.h_{1}(1400)\right)$. When we are only talking about two states. we can represent the mixing by a rotation:

$$
\left(\begin{array}{l}
h_{1}(1270)^{+} \\
h_{1}(1400)^{+}
\end{array}\right)=\left(\begin{array}{cc}
\cos \theta & \sin \theta \\
-\sin \theta & \cos \theta
\end{array}\right)\left(\begin{array}{l}
h\left({ }^{1} P_{1}\right)^{+} \\
h^{3}\left({ }^{3} P_{1}\right)^{+}
\end{array}\right) .
$$

For this particular mixing. we take $\theta=45^{\circ}$ [1] for the calculations of Section 3.2. In Section 3.3, we attempt to determine this mixing angle by comparing predictions of meson decay models to experimental data.

In terms of the amplitudes and widths. Eq. E.2 becomes with mixing. using a decay involving the $h_{1}(1270)^{+}$for example.

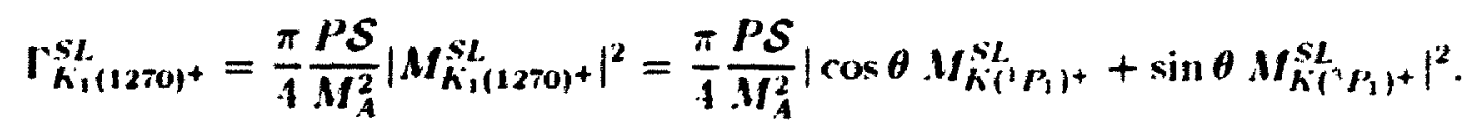

In Eq. A.5. note that we specified the positively charged mesons. because the flawour makes a difference to the mixing angle for these particular mesons. This comes about in the quark model Hamiltonian - the term that causes the mixing. $\left\langle H_{q 4}^{\text {so- }}\right\rangle$. changes sign when the antiquark instead of the quark is the heavier st range. and this leads to a mixing angle of opposite sign when the ${ }^{1} P_{1}{ }^{3} P_{1}$ Hamiltonian is diagonalized (see Eys. 3.3 and 3.4). Thus if we have a mixing angle of $45^{\circ}$ for the $K_{1}(1270)^{+}$and $K_{1}(1400)^{+}$(and $K_{1}(1270)^{\prime}$ and $K_{1}(1400)^{\circ}$ ). we must use $-45^{\circ}$ for the $h_{1}(1270)^{-}$and $h_{1}(1400)^{-}$(and $k_{1}(1270)^{\circ}$ and $\left.k_{1}(1400)^{\circ}\right)$. 
The other meson mixing that we use in this work' is due to the tensor part of the colour-hyperfine interaction [H]:

$$
\left(\begin{array}{l}
h \cdot(1410) \\
h \cdot(1680)
\end{array}\right)=\left(\begin{array}{cc}
1.00 & 0.04 \\
-0.04 & 1.00
\end{array}\right)\left(\begin{array}{l}
h\left(\cdots \cdot s_{1}\right) \\
h\left(1^{3} J_{1}\right)
\end{array}\right)
$$

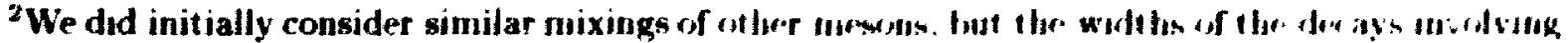
these mesons were too small to be included in cur rasults
} 


\section{Appendix B}

\section{Meson Wavefunctions Used in this}

\section{Work}

As can be seen from Eq. 2.1. meson wavefunctions contain four different componemt wavefunctions: space. spin. flavour and colour. The space and flavour wavefunctions are given below: The spin wavefunctions are given in Appendix D.3. The colour wavefunction is trivially a colour singlet (see Appendix D.1).

\section{B.1 Space Wavefunctions}

In this work we investigate three different sets of meson space wavefunctions. though only two are used for calculations. All three are expressed in terms of simple harmonic encillator (SllO) wavefunctions. In moment um-space the SHO wavefunctions are

$$
\left.\underset{n L H_{L}}{\mathrm{SHO}}(\vec{p})=R_{n !}^{\mathrm{SHO}}(p)\right)_{L M_{I}}\left(\Omega_{t_{1}}\right)
$$


where the radial wavefunctions are given by

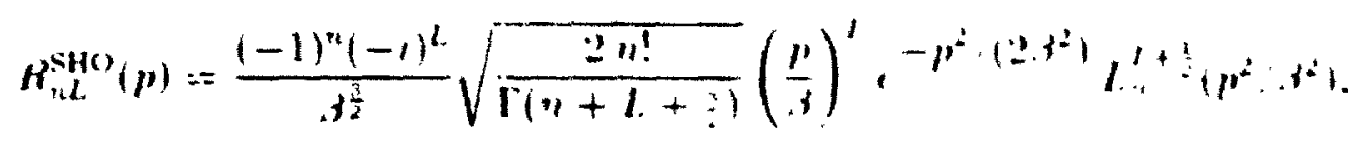

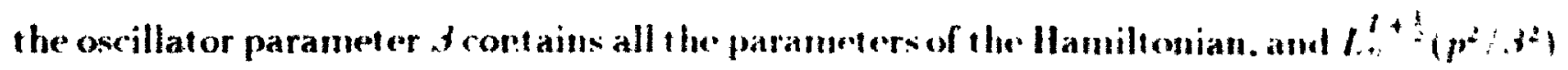
is an associated Laguerre polynomial. The quantum numbers $"$ und $I$ are unrelatol. and have the ranges: $m: 0.1 .2 \ldots$ and $l: 0.1 .2 \ldots$

In position-space we have the Fourier transform of the aluse.

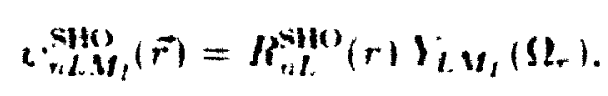

where the radial wavefunctions are given by

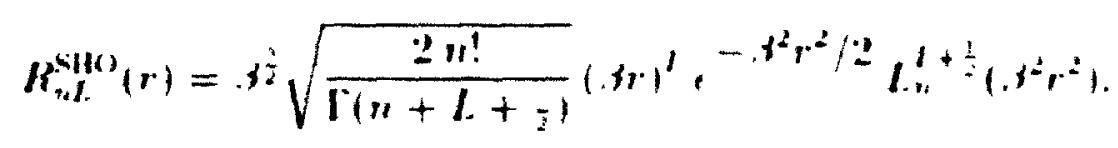

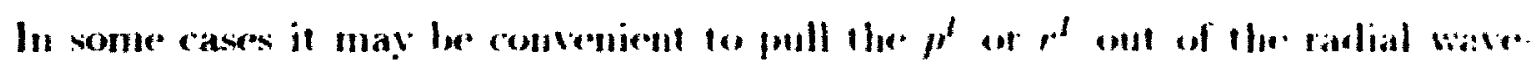

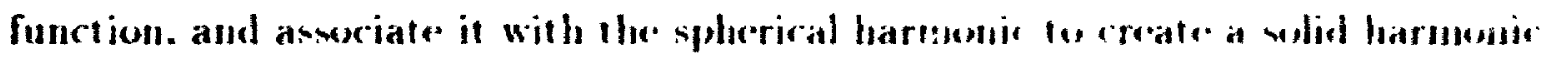

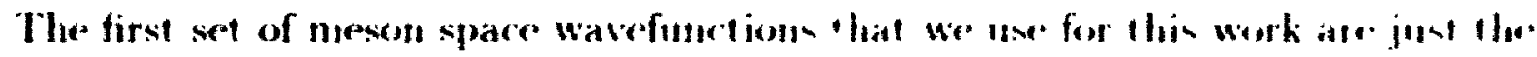

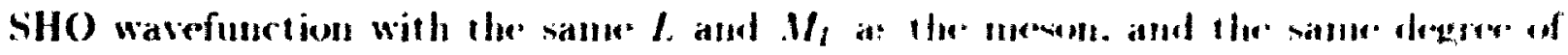

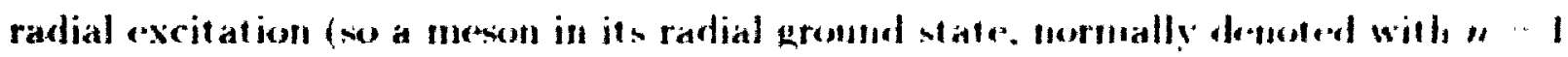

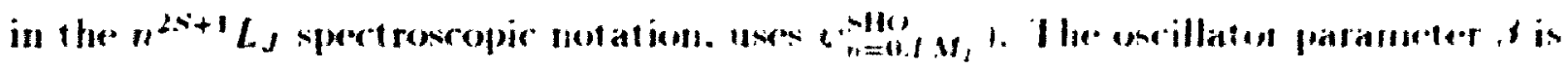

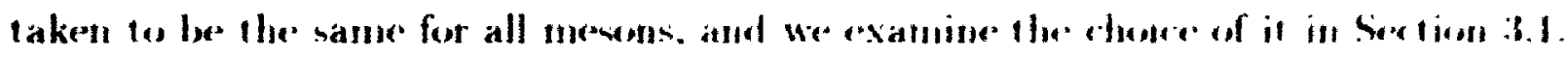

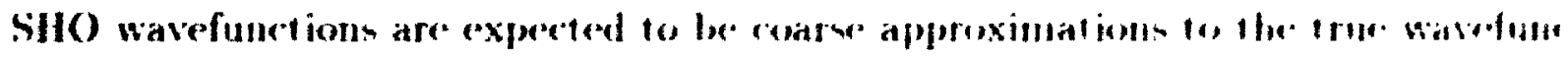
tious. since they neglect all but the limeat contining part of the menen potentiat. and

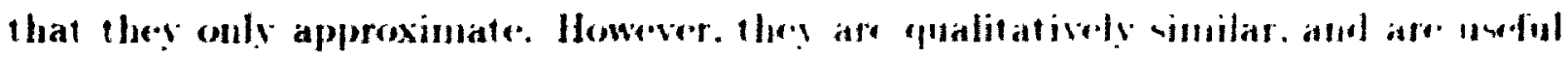
for generating analytical results.

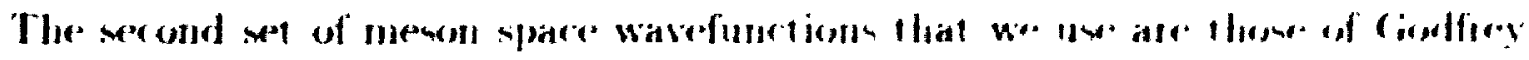

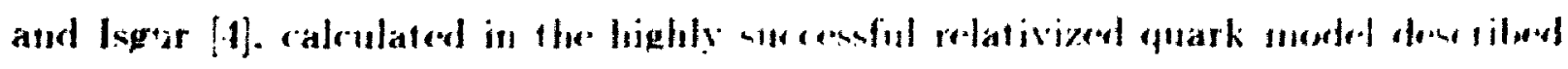


in Sertion 1.2.2. We will label these watefunctions by RQM. The radial parts of these wavefunctions are expressed as linear combinations of the first $l+1$ radial

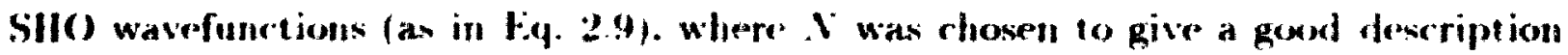
of the partirular wavefunction. The oscillator pasameter was fit individually for each mesem, and the quark massen were also fitted: $m_{\mathrm{y}}=220 \mathrm{Mel} . m_{d}=220 \mathrm{Mlel}$. and $m_{4}=41 ! \mathrm{M} \cdot \mathrm{l}$.

The third set of meson space wavefunctions that we use are single SHO watefunctions like the first set. but with the effertive osrillator parameters (differest for each mesonf of Kokoski and lsgur [5]. The effertive parameters were obnained ley fitting the rms moment of the SHO wavefunctions to be equal to those obtained from the serond set of wavefunctions. Wi do not use this third set of wavefunctions for actual calculations besause of their peor performance in section 3.1 .

\section{B.2 Flavour Wavefunctions}

Wr use the following meson flavour wavefunctions in this work:

For the isovertors:

$$
\begin{aligned}
& \pi^{+}=-i d d \\
& \pi^{\prime \prime}=\frac{1}{\sqrt{2}}(u n-d d) \\
& \pi^{-}=d u .
\end{aligned}
$$

For the st range g'tesons:

$$
\begin{aligned}
& \boldsymbol{k}^{-+}=-\boldsymbol{\omega} \\
& \boldsymbol{k}^{-0}=-d \omega \\
& \boldsymbol{k}^{-0}=-\boldsymbol{d}
\end{aligned}
$$




$$
\boldsymbol{h}^{-}=\mathbf{N} \text {. }
$$

For the isoscalars we assumed -idloal mixing".

$$
\begin{aligned}
& c_{\text {munNirange }}=\frac{1}{1:}(u u+d u) \\
& \text { Qnetenger }=\ldots \text {. }
\end{aligned}
$$

except for the pseudoscalars in a radial ground stale. Where we assument "gmetent mixing".

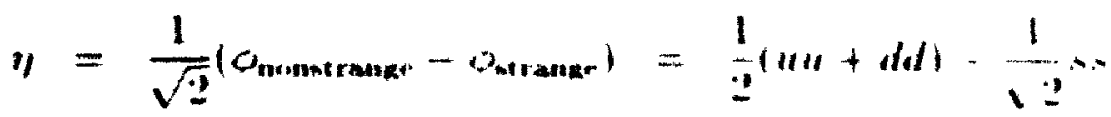

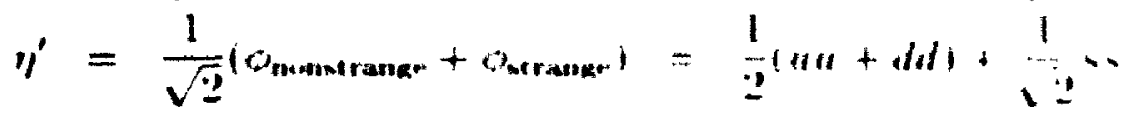

In terms of the SI (3) flavour reprevent ation [1].

$$
\begin{aligned}
& \eta=1 / \cos \theta_{l}-1 / 1 \sin \theta_{l} \\
& y^{\prime}=1 / x \sin \theta_{l}+1 / \cos \theta_{l}
\end{aligned}
$$

where*

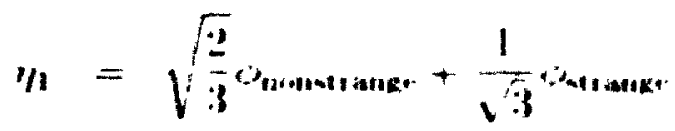

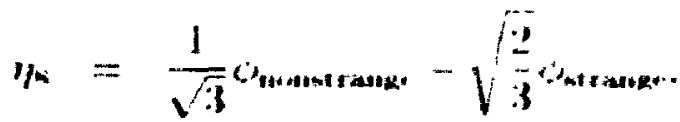

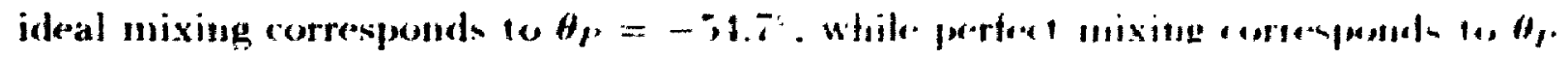

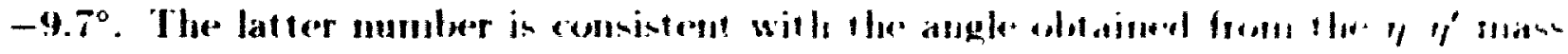

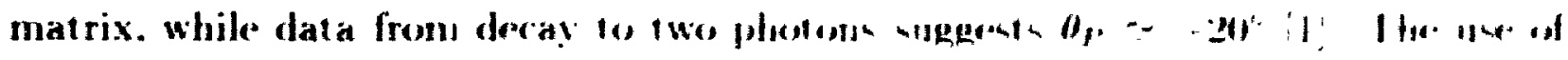
this second value is considered in ('haphen 3 . 


\section{Appendix C}

\section{Field Theory Conventions Used in this Work}

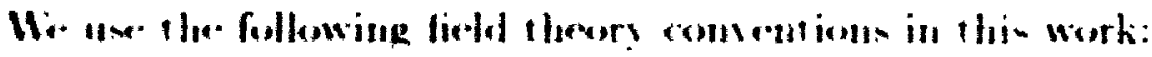

for the spinor mormalizations.

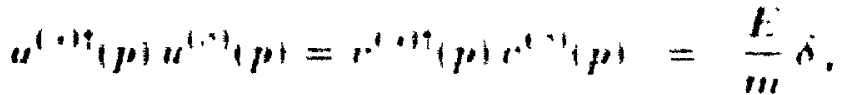

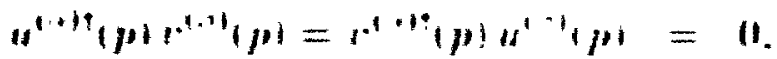

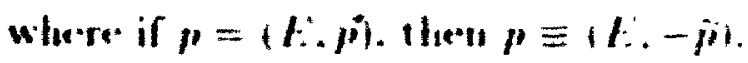

Wi detine the upinor fiedd

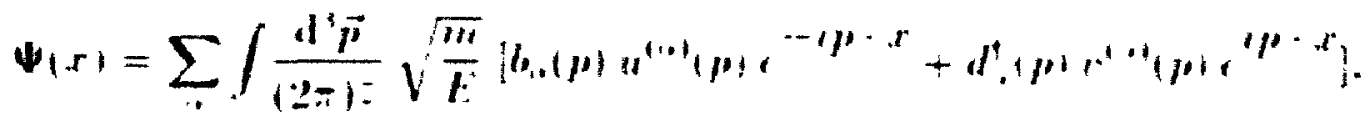

and use the ant icommutators

$$
\begin{aligned}
& \left\{\boldsymbol{\Psi},\left\{t \cdot \vec{x} \cdot, \boldsymbol{\Psi}^{*}\left(t, \vec{x}^{\prime}\right)\right\}=s^{2} \cdot \vec{x}-\vec{r}^{\prime} \mid d\right.
\end{aligned}
$$

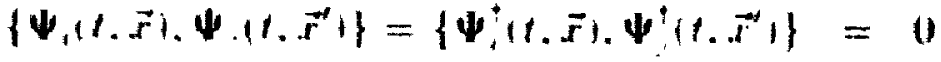


Appendix (: Field Therory (innention- I wd in thi- llith

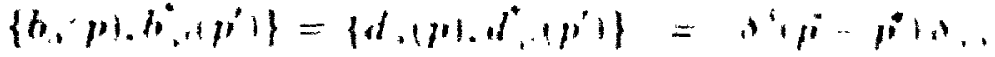

$$
\begin{aligned}
& \left.\left\{b,(p), b,\left(f^{\prime}\right)\right\}=\left\{d,(n), d, \cdot p^{i}\right)\right\}=\|
\end{aligned}
$$

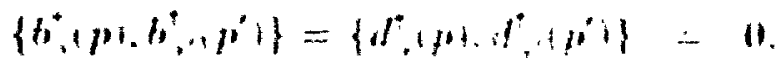

which gives for the normalization of ence particle vatem

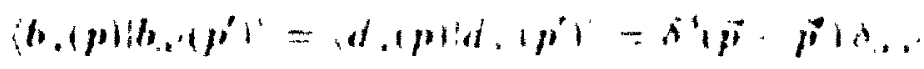




\section{Appendix D}

\section{Evaluating the Colour, Flavour}

\section{and Spin Overlaps for Models of}

\section{Meson Decay}

In the ${ }^{3} P_{0}$ and flux-tube breaking models of meson decay, the overlaps of the colour. flavour. spin and space wavefunctions of the mesons and the created pair must be calculater. The overlap of the space wavefunctions is accomplished by the integrals of Eys. 2.8 and 2.23. The other overlaps are discussed below.

\section{D.1 Colour Overlap}

The calculation of the colour overlap is particularly simple. because the mesons and the created pair are all colour singlets. with the wavefunction $\omega=\frac{1}{\sqrt{3}}\left(R \bar{R}+G_{i} \bar{G}+B \bar{B}\right)$ (using the arbitrary three colours Red. (ireen. Blue).

This may be represented in matrix form (this may seem excessive now. but will 
be useful for the flavour overlaps as well) by

$$
\dot{L}=\frac{1}{\sqrt{3}}\left(\begin{array}{lll}
1 & 0 & 0 \\
0 & 1 & 0 \\
0 & 0 & 1
\end{array}\right)
$$

where the rows indicate a quark of colour $R$. $i_{i}, B$, and the columns indicate antiquarks of colcr $\boldsymbol{R}_{.}, \dot{G}, \hat{\boldsymbol{B}}$.

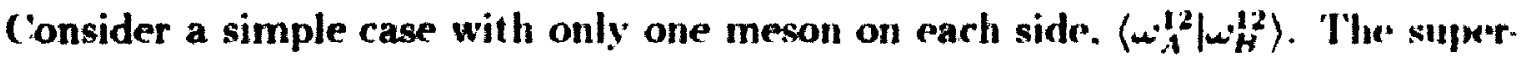
srripts indicate that the quark of $A$ (labelled 1 ) is also the quark of $B$. and similarly for the antiquark (labelled 2). A particular colour combination (e.g. $I R$ ) will only contribute to the overlap if it is found on both sidess of the bra-ket. and the resulting term is just the product of the coefficients in the mat rices for that colour combination. Then the total result is just the sum of these terms over the possible conours.

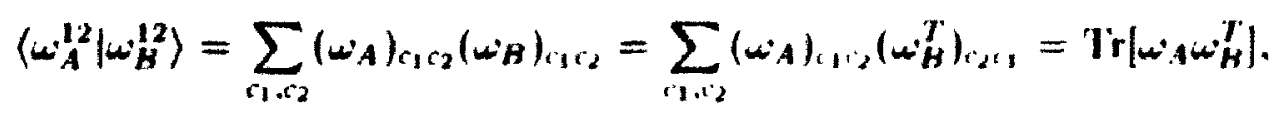

where $c_{1}\left(c_{2}\right)$ runs over the quark (antiquark) colours.

This is easily expanded to the case where there are two mesons on each side (e.g. for the first diagram of Figure 2.1 ):

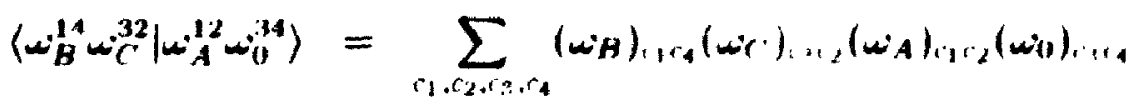

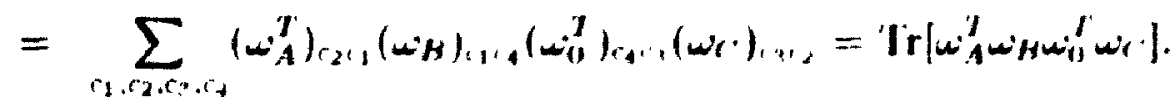

For the case where everything is a colour singlet. the results are

$$
\left\langle\omega_{B}^{14} \omega_{i}^{32} \mid \omega_{1}^{12} \omega_{i 1}^{34}\right\rangle=\left\langle\omega_{H}^{32} \omega_{1}^{34} \mid \omega_{1}^{12} \omega_{i j}^{34}\right\rangle=\frac{1}{3} .
$$




\section{D.2 Flavour Overlap}

The overlap of the flavour wavefunctions of the mesons and the created pair can be calculated using the matrix notation introduced in Appendix D.1. The flavour of a meson may be represented by a matrix where the rows indicate a quark of Gavour $u$. $d, s, \ldots$ and the columns indicate antiquarks of color $\bar{u} . \bar{d}, \bar{s}, \ldots$ For example. consider the $\pi^{0}$,

$$
\phi_{\pi^{0}}=\frac{1}{\sqrt{2}}(u \bar{u}-d \bar{d})=\frac{1}{\sqrt{2}}\left(\begin{array}{ccc}
1 & 0 & 0 \\
0 & -1 & 0 \\
0 & 0 & 0
\end{array}\right) .
$$

and the $\mathrm{K}^{++}$.

$$
\phi_{h+}=-u \bar{s}=\left(\begin{array}{ccc}
0 & 0 & -1 \\
0 & 0 & 0 \\
0 & 0 & 0
\end{array}\right)
$$

The ${ }^{3} P_{0}$ and flux-tube breaking models assume that the pair is created in an SII(N) flavour singlet; we take $N=3$, since we are not concerned with any mesons involving $c, b$ or $t$ quarks:

$$
\phi_{0}=\frac{1}{\sqrt{3}}(u \bar{u}+d d+s \bar{s})=\frac{1}{\sqrt{3}}\left(\begin{array}{ccc}
1 & 0 & 0 \\
0 & 1 & 0 \\
0 & 0 & 1
\end{array}\right)
$$

Taking $\mathrm{N}$ to be some ot her number would change the value of $\gamma\left(\gamma_{0}\right)$ needed to fit the data (because of the normalization in Eq. D.7). but would not change the calculated decay widths.

The overlap is given by expressions similar to Eq. D.3:

$$
\left\langle\phi_{B}^{14} \phi_{C}^{32} \mid \phi_{A}^{12} \phi_{0}^{34}\right\rangle=\operatorname{Tr}\left[\phi_{A}^{T} \phi_{B} \phi_{0}^{T} \phi_{C}\right]=\frac{1}{\sqrt{3}} \operatorname{Tr}\left[\phi_{A}^{T} \phi_{B} \phi_{C}\right]
$$


Appendix D. Evaluating the Colour. Flavour and Spin Overlaps...

$$
\left\langle\phi_{B}^{32} \phi_{C}^{14} \mid \phi_{A}^{12} \phi_{0}^{34}\right\rangle=\operatorname{Tr}\left[\phi_{A}^{T} \phi_{C} \phi_{0}^{T} \phi_{B}\right]=\frac{1}{\sqrt{3}} \operatorname{Tr}\left[\phi_{A}^{T}{\phi_{C}}_{C H}\right] .
$$

In many cases only one of these overlaps will be non-zero.

As an example. consider the flavour overlaps in the decay $h^{*}(8 ! 12)^{+} \rightarrow h^{+} \pi^{\prime \prime}$ : $\phi_{K \cdot(892)^{+}}=\phi_{K^{+}}$, giving us

$$
\begin{aligned}
& \left\langle\phi_{B}^{14} \phi_{C}^{32} \mid \phi_{A}^{12} \phi_{0}^{34}\right\rangle=0 \\
& \left\langle\phi_{B}^{32} \phi_{C}^{14} \mid \phi_{A}^{12} \phi_{0}^{34}\right\rangle=\frac{1}{\sqrt{6}} .
\end{aligned}
$$

\section{D.3 Spin Overlap}

Since quarks have spin $\frac{1}{2}$. their spins may only combine to give 10 of 1 in a meson. They combine in the usual singlet and triplet states (given as e.g. $\left.\left|S_{A}, M_{S_{A}}\right\rangle\right)$ :

$$
\begin{aligned}
& |00\rangle=\frac{1}{\sqrt{2}}\left[\left(\frac{1}{2} \frac{1}{2}\right)\left|\frac{1}{2}-\frac{1}{2}\right\rangle-\left(\frac{1}{2}-\frac{1}{2}\right) \mid\left(\frac{1}{2}\right)\right] \\
& \left\{\begin{aligned}
|11\rangle & =\left|\frac{1}{2} \frac{1}{2}\right\rangle\left|\frac{1}{2} \frac{1}{2}\right\rangle \\
|10\rangle & =\frac{1}{\sqrt{2}}\left[\left|\frac{1}{2} \frac{1}{2}\right\rangle\left|\frac{1}{2}-\frac{1}{2}\right\rangle+\left|\frac{1}{2}-\frac{1}{2}\right\rangle\left|\frac{1}{2}\right\rangle\right] . \\
|1-1\rangle & =\left|\frac{1}{2}-\frac{1}{2}\right\rangle\left|\frac{1}{2}-\frac{1}{2}\right\rangle
\end{aligned}\right.
\end{aligned}
$$

The overlap of the spin wavefunctions of the mesons and the creatied pair and be calculated by angular moment um algebra. It can be expanded in a sum over persibly. total spins (e.g. for the first diagram of Figure 2.1)

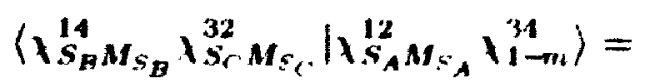

$$
\begin{aligned}
& \sum_{S, M_{S}}\left(\left(j_{1} j_{4}\right) S_{B} \cdot\left(j_{3} j_{2}\right) S_{C}: S M_{S} \mid\left(j_{1} j_{2}\right) S_{A} \cdot\left(j_{B, j_{1}}\right) 1: S H_{S}\right) \\
& \times\left\langle S_{B} M_{S_{B}} S_{C} \cdot M_{S_{C}} \mid S M_{s}\right\rangle\left\langle S_{A} M_{s_{A}}|-m| S M_{s}\right\rangle
\end{aligned}
$$


where the $j_{\mathrm{t}}$ 's are all $\frac{1}{2}$, but are necessarily written this way to show the connection between the left and right sides of the first factor. The first factor is a transformation between two different bases for combining four angular momenta. and is independent of $M_{s}$. On the left side, $j_{1}$ and $j_{4}$ are added together to obtain $S_{B}$. which is added to

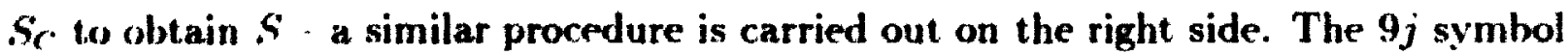
is defined in terms of this transformation.

$$
\begin{aligned}
& \left\langle\left(j_{1} j_{4}\right) J_{14},\left(j_{3} j_{2}\right) J_{32} ; J M \mid\left(j_{1} j_{2}\right) J_{12} \cdot\left(j_{3} j_{4}\right) J_{34}: J M\right\rangle= \\
& (-1)^{2 J_{12}+2 J_{14}+j_{2}-J_{4}+J_{32}-J_{34}} \sqrt{\left(2 J_{12}+1\right)\left(2 J_{34}+1\right)\left(2 J_{14}+1\right)\left(2 J_{32}-1\right)} \\
& \times\left\{\begin{array}{ccc}
j_{1} & j_{2} & J_{12} \\
j_{4} & j_{3} & J_{34} \\
J_{14} & J_{32} & J
\end{array}\right\} .
\end{aligned}
$$

which allows us to write the spin overlap in terms of a $9 j$ symbol:

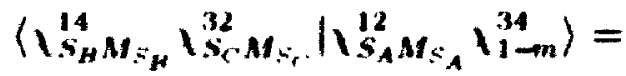

$$
\begin{aligned}
& (-1)^{s_{C}+1} \sqrt{3\left(2 S_{A}+1\right)\left(2 S_{B}+1\right)\left(2 S_{C}+1\right)} \sum_{S . M_{B}}\left(S_{B} M_{S_{B}} S_{C} \cdot M_{S_{A}}\left|S S_{S}\right\rangle\right. \\
& \times\left\langle S_{A} M_{S, 1-m\left|S M_{S}\right\rangle}\left\{\begin{array}{ccc}
\frac{1}{2} & \frac{1}{2} & S_{A} \\
\frac{1}{2} & \frac{1}{2} & 1 \\
S_{H} & S_{S} & S
\end{array}\right\}\right. \text {. }
\end{aligned}
$$

I'sing an alternative definition of the $9 j$ symbol that couples the quarks different ly. we a an get a similar expression for the spin overlap for the second diagram of Figure 2.1 which satisfies

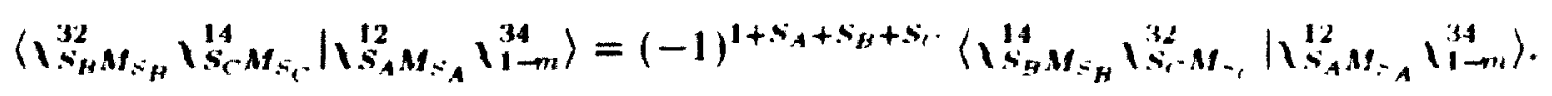


Appendix D. Evaluating the Colour. Flavour and Spin Overlaps...

Eq. D.15 was used to simplify. Eq. 2.7. 


\section{Appendix E}

\section{Converting to Partial Wave}

\section{Amplitudes for Models of Meson}

\section{Decay}

The deray amplitudes of the ${ }^{3} P_{0}$ and flux-tube breaking models derived in Sections 2.1 and 2.3. $M^{M J_{A} M J_{B} M J_{r}}$, are given for a particular basis of the final state: $\mid \theta$. o. $\left.M_{J_{B}}, M_{J_{C}}\right\rangle \equiv\left|\Omega . M_{J_{B}}, M_{J_{c}}\right\rangle$. Here $\theta$ and $\phi$ are the spherical polar angles of the cutgoing momentum of meson $B\left(\vec{P} \equiv \vec{P}_{B}\right)$ in the CM frame.

We would prefer to calculate amplitudes for particular outgoing partial waves. $|J, M . S, L\rangle$. since they are what are measured experimentally. Here $|J . M\rangle$ are the quantum numbers of the total angular momentum of the final state. $\left|S . M_{s}\right\rangle$ are the quant um numbers for the sum of the total angular momenta of $B$ and $C$. and $\left|L . . H_{L}\right\rangle$ are the quantum numbers for the orbital angular moment um between $B$ and $($.

The formula for the decay width in terms of partial wave amplitudes is different from Eq. 2.5:

$$
r=\sum_{s, L} r^{s L}
$$


where

$$
r^{S I}=\frac{\pi}{1} \frac{P \mathcal{S}}{1 M_{1}^{2}}\left|M^{S L}\right|^{2}
$$

$M^{S L}$ is a partial wave amplitude, and $\Gamma^{S L}$ is the partial width of that partial wave.

We use two methods to convert our calculated amplitudes to the partial wave basis [69]: by use of a recoupling calculation. and hy use of the Jacob-Wick Formula.

\section{E.1 Converting by a Recoupling Calculation}

The recoupling transformation directly relates the bases $\left|\Omega . M_{J_{I \prime}}, I_{J_{1}}\right\rangle$ and $\left.\mid J_{2}, I, . S, I\right\}$ :

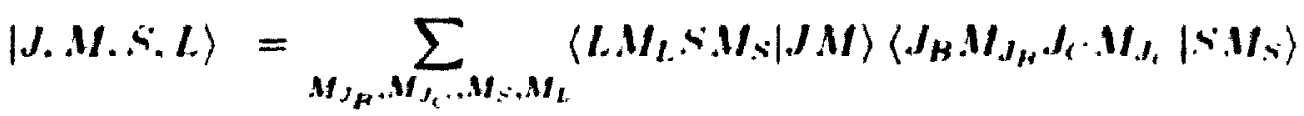

$$
\begin{aligned}
& \times \int d \Omega Y_{L M_{L}}(\Omega) \mid \Omega\left(M_{J_{B}}, M_{J_{C}}\right\rangle
\end{aligned}
$$

In terms of the amplitudes. the net result we want is the transformation

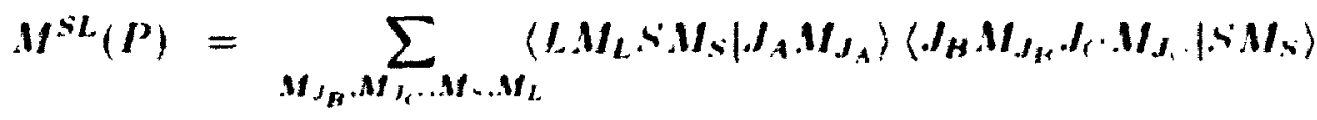

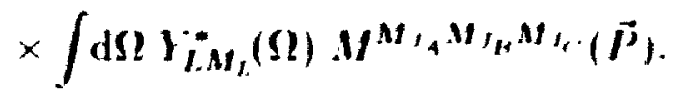

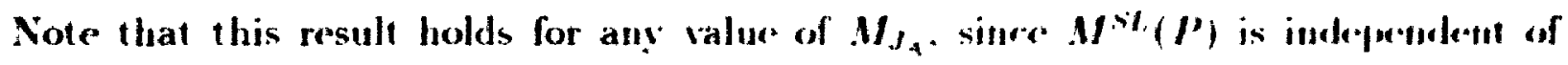
$\boldsymbol{M}_{J_{A}}$. If we wanted to avoid facouring one particular value. we comlal sum wer $I_{I_{A}}$. and convert the sum on the left side to a factor of $1 /\left(2 . I_{A}+1\right)$ an the right sidk. 


\section{E.2 Converting with the Jacob-Wick Formula}

The Jaroi-Wick formula

$$
|J . M, S, L\rangle=\sum_{\lambda_{B}, \lambda_{C}} \sqrt{\frac{2 L+1}{2 J+1}}\langle L 0 . S \lambda \mid . J \lambda\rangle\left\langle J_{B} \lambda_{B} J_{C} \cdot-\lambda_{C} \mid . S \lambda\right\rangle\left|J ., I . \lambda_{B}, \lambda_{C}\right\rangle
$$

relates the partial wave basis $|J, M, . S, L\rangle$ to the helicity basis $\left|J, M, \lambda_{H}, \lambda_{r^{\prime}}\right\rangle$. where $\lambda_{H}$ and $\lambda_{c}$, are the helicities of $B$ and $(\cdot$, respectively. To use it we must firsi relate the helicity basis to the basis $\left|\Omega . \lambda_{B} . \lambda_{C}\right\rangle$.

$$
\left|J . M, \lambda_{B}, \lambda_{C}\right\rangle=\sqrt{\frac{2 . I+1}{4 \pi}} \int d \Omega D_{A i,}^{\prime *}(0, \theta .0)\left|\Omega, \lambda_{B} \cdot \lambda_{C}\right\rangle
$$

where $D_{A f}^{d_{i}}(\phi, \theta, 0)$ is a rotation matrix. and then choose $\vec{P}$ to lie along the positive $z$ axis (in the (M frame still), so that $\lambda_{B}=M M_{J_{B}} . \lambda_{C}=-M_{J_{C}}$. and $\left|\Omega . M_{J_{P}} . M_{J_{C}}\right\rangle=$ $\left|\Omega, \lambda_{H},-\lambda_{C}\right\rangle$.

The final transformation betwen the amplit udes is

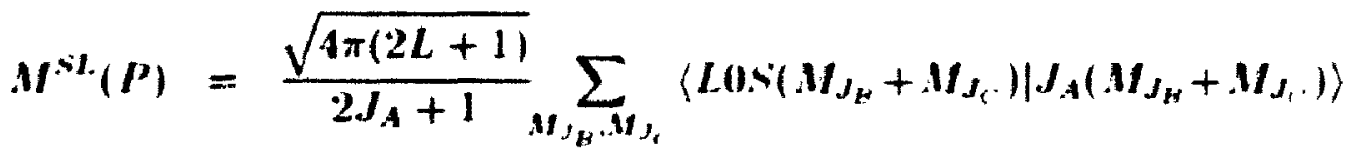

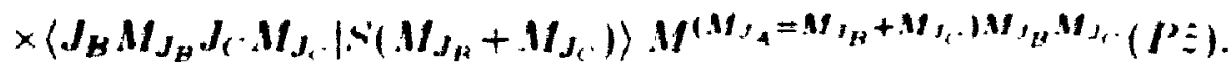

Note that $M_{J_{A}}$ in the calculated amplitude is replaced by $M_{J_{f}}+I_{J_{1}}$. 


\section{Bibliography}

[1] Particle Data Group. L. Montanet at al. "Review of Particle Propert ies". 1"hyss. Rev. D) 35. 1173 (1994) and 1995 off-year partial updnte for the lame colition available on the PDG; WWW pages (1KL: hatp://pdg.llh.gow/l.

[2] John hogut and Leonard Susskind. Physs. Rev. 1) 11, 3!9:. (1!nti).

[3] Nathan Isgur and Jack Paton, Phy's. Rev. 1) 31. 2910 (195i).

[1] Stephen Godfrey and Nathan Isgur. Physs. Rev. I) 32. I X!! (I!NKi).

[i] Richard Kokoski and Nathan Isgur. Phỵs. Ro.v. D) 35. 9617. (19ki).

[6] Harry (a. Hlundell and Stephen Godfrey. Phys. Rev. D) 53. 37(M) (IMMi).

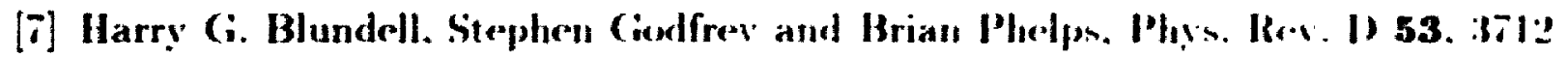
(1996).

[S] The ${ }^{3} P_{0}$ model of meson decay was first discussed by A. L. Yamatic. I. (Niver.

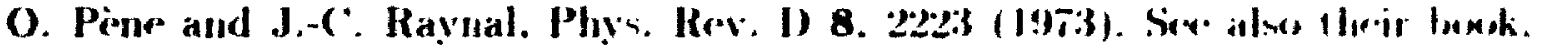

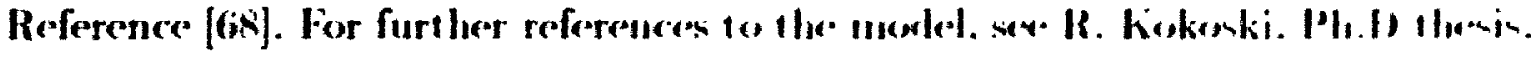
Iniversity of Toronto. 1984.

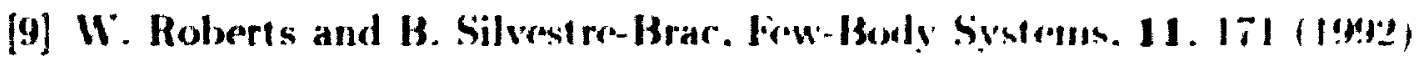

[10] Cameron Hayne and Nathan Isgur. Phys. Rex. 1) 25. 1914 (19):2. 
[11] Rirhard N. Zare, Angular Momentum: Pnderstanding Spatial Aspects in (bermistry and Physics (John Wiley $\mathrm{d}$. Sons. New York. 1988).

[12] Strphen Wolfram. Mathematira: A System for Doing Mathematirs by Computor. 2nd ed. (Addison-Wesley, Redwood (ity, 1991). For the flux-tube breaking model of meson decay. FOKTKAX cole was created using the Mathomatica packages Format.m and Optimize.m. written hy M. Sofroniou and available from MathSource (VRL: http://www.wri.com/).

[1:3] (i. Peter Lepage. J. (omput. Phys. 27. 192 (19ix): WEGAS - An Adaplive Multi-dimensional Integration Program". Cornell Cniversity. 'Teclinical Note (I.NS-80/447, 1980.

[1.1] Paul Cieiger and Eric S. Swanson. Phys. Kev. D 50. 685is (1994).

[15] Stephen Godfrey (private communication: Nathan Isgur (private communica(ion)).

[16] MAlk III Collaboration. First reported in a presentation by K.f. Einsweiler. International Europhysics Conference on High Energy Physics. Brighton. I'.K.. July 20-27. 1983: final analysis presented in K.M. Baltrusait is et al.. Plị.s. Rev. Lett. 56. 107 (1986).

[17] R. Michael Barnett. (ioran Senjanovic. L. Wolfenstein, and I). Wỵter. Phys. Lett. B 136. 191 (198.1): K.S. Willey: Phy's. Rev. Lett. 52. 5kis (1984): Howard E. Haber and (i.l. Kane. Plins. Lett. B 135. 196 (1984).

[18] Michael P. Shatz. Phys. Lett. B 138. 209 (1984).

[19] Kuang-Ta ( hao. Commun. Theor. Phys. 3. 757 (1981).

[20] Sandip Pakvasa, Mahiko Suzuki and S.F. Tuan. Phys. Lett. B 145. 135 (1984). 
[21] Seiji Ono. Phỵs. Rev. D 35. 944 (193i).

[22] Michael S. Chanowitz and Stephen R. Sharpe. Mly!s. Letl. H 13. M13. (16:3).

[23] Kuang-Ta (hao. Commun. Theor. Phys. 24, 373 (144:3).

[24] P.D. Barnes of al. Physs. Lett. B 309. 464 (19913).

[20] Serpukhor-Brussels-Los Alamon Anmecy (L.APl) ('ollatoration, 1). Nhk el at. Phys. Lett. B 177. 120 (1986).

[26] B.l. Bolonkin at al. Nucl. Phys. B 309. 42t (19k8).

[27] D. Aston $t$ al.. Phỵs. Lett. B 215. IMU (19kא).

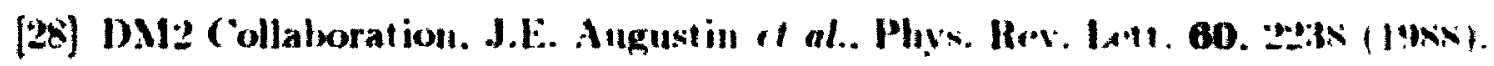

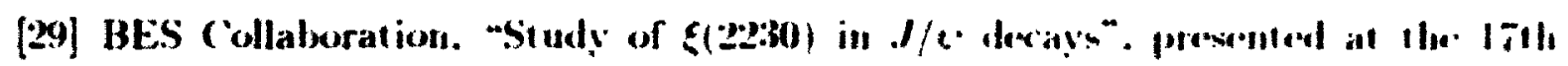
International Symposium on Lepton Photon Interactions. Majiung. Angust II 15. 1995.

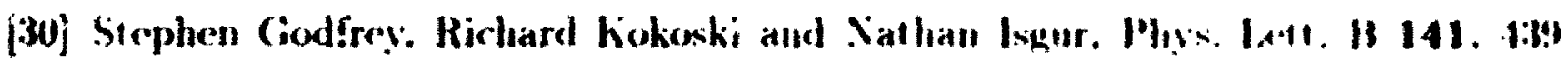
(1984).

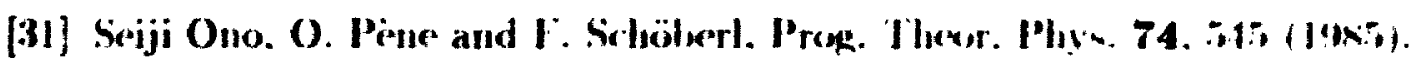

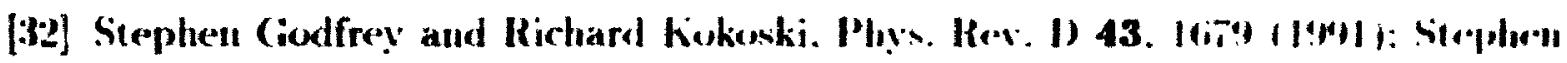
Godfrey (private communication).

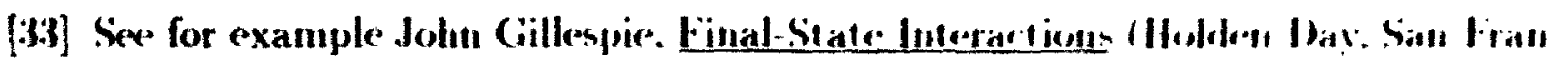
cisco. 19641$)$.

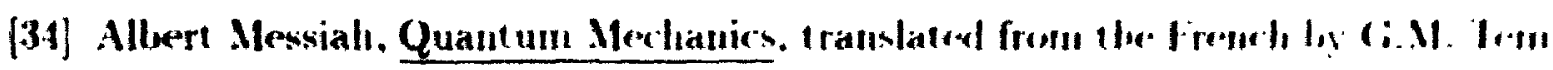

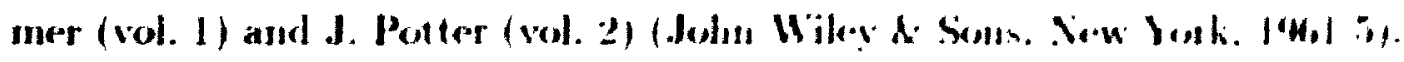


[3.1) Enrico Fermi. Elementary Particlem (Yale Iniversity Press. New Haven. 1951).

[36] A. Couran of al. Nucl. Phys. H 271.1 (1986).

[37] F.F. Low, Plyss. Kov 96. 142k (1951): M. (iell-Mann and M.L. Cioldberger, H'hys. Rm. 96. 1.1333 (1954): Henry 1).1. Abarbanel and Marvin L. Coldberger. Plyss. Rev. 165. 1594 (196is).

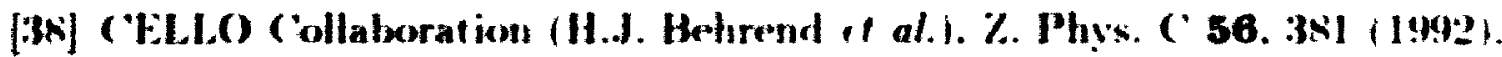

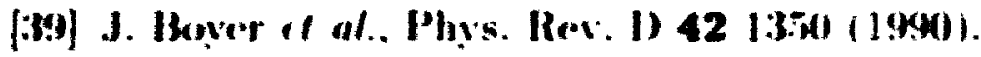

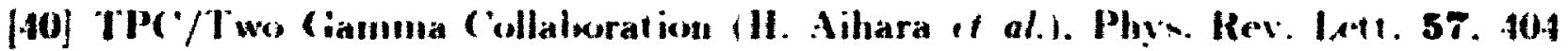
(19)(i).

Hil Some of the dath shown for the $9 \rightarrow \rightarrow \pi$, work was downloaded from the Durbam/RAL. HEP Reaction Data Database at htep://durpdg.dur.ar.uk/HEPDATA.

|42! Some of the data shown for the $99 \rightarrow \pi-5$ work wa obtained from D. Morgan. M.K. Pennington and M.K. Whalley. J. Phys. (; 20. Al 11991 .

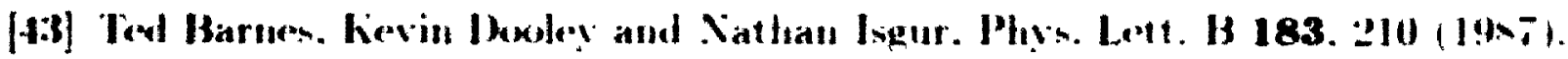

[4.1) W. Morgan and M.R. Penmington. Phe. Lett. Is 272.131 119911.

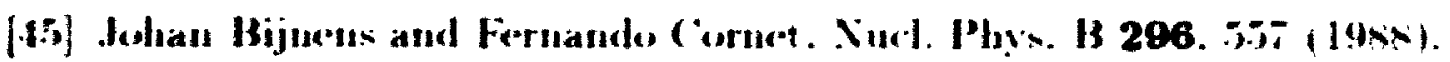

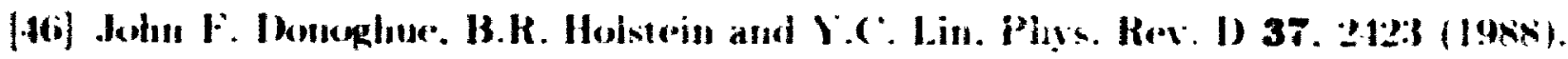

[47] S. Bellucri, J. (asserer and M.E. Sainic. Nucl. Ply's, H 423. No (1994): Erratum 431. $113(1941)$.

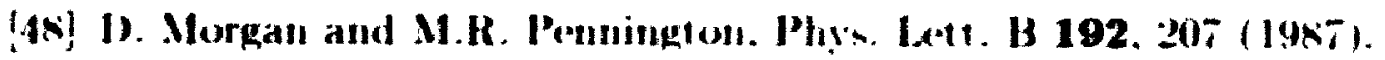


[49] John F. Donoghue and Barry R. Hoistein. Mhys. Hev. I) 48. 1:56 1:M:31

[50] Eric S. Swanson. Ann. Phy. (X.) 220 . .3 (1!n:2).

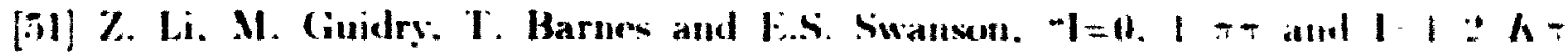

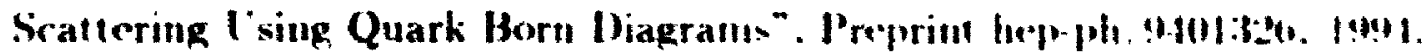

[52] Eric S. Swanson (private connmunication).

[53] T. Barnes and E.S. Swanson. Phys. Mex. 1) 46. 131 1 I6911).

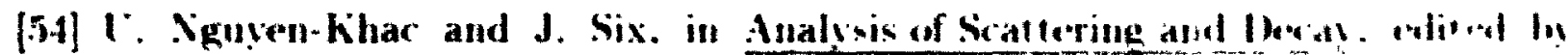
II. Nikulic (Cordon and Breach. New York. Ithix).

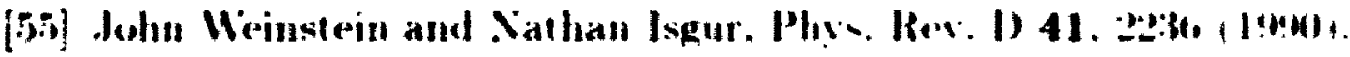

[56] John Weinstein. The Multichanned Quark Model". Preprim I MIIIt' 'Mo 10:1.

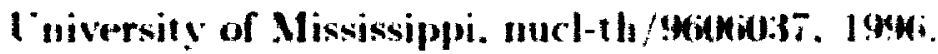

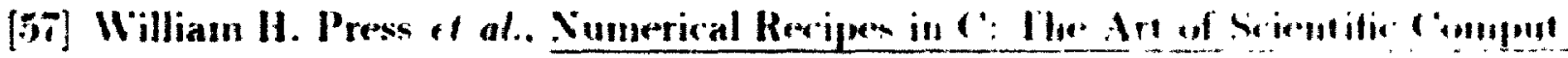

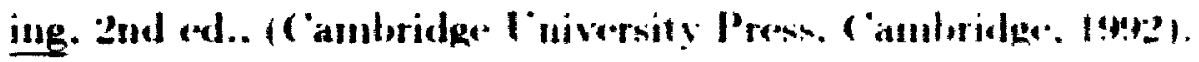

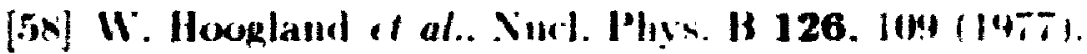

[59] J.P. Prukof at al. Phy, Rev. I) 10.2055 (1951).

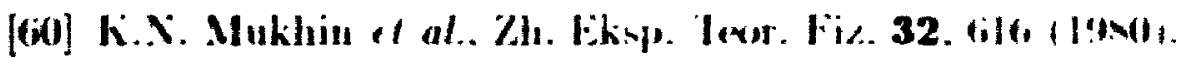

[61] L. Rosselet at al. Physs. Ret. 1) 15. i7 (1977i.

[62] P. Estabrooks and A.D. Nartin. Nucl. P'in. I3 79. 3(1) 1931 ,

[6.3) S.1). Protopopesen t al. Phys. Rev. 1) 7. 1279) 11973, 
[64] Crystal Ball Collaboration (J.K. Bienlein for the collaboration) in Proceedings of the IX International Workshop on Photon-Photon Collisions. edited by. D. Caldwell and II.P. Paar (World Scientific. Singapore. 1992). p. 241.

[65] Crystal Ball ('ollaboration (H. Marsiske al al.). Phys. Rev. D 41.3324 (1990).

[66] ('. Edwards ot al., Phys. Lett. B 110. 82 (1982).

[67] David (iriffiths, Introduction to Elementary Particles (John Wiley \& Sons. New York, 198i).

[68] Alain le Yaouanc, Lluis Oliver. Olivier Pène and Jean-Claude Raynal. Hadron Transitions in the Quark Model (Gordon and Breach Science Publishers. Now York, 1988).

[69] Suh tirk (hung, "Spin Formalisms". Technical Note ('ERN i1-s, CERN. 1971: Jaffrey D. Richman. "An Experimenter`s Guide to the Helicity Formalism”. Technical Note (ALT-68-1148. California Institute of Technology. 1984: M. Jacob and (i.C. Wick. Aun. Phys. 7 40.4 (1959). 

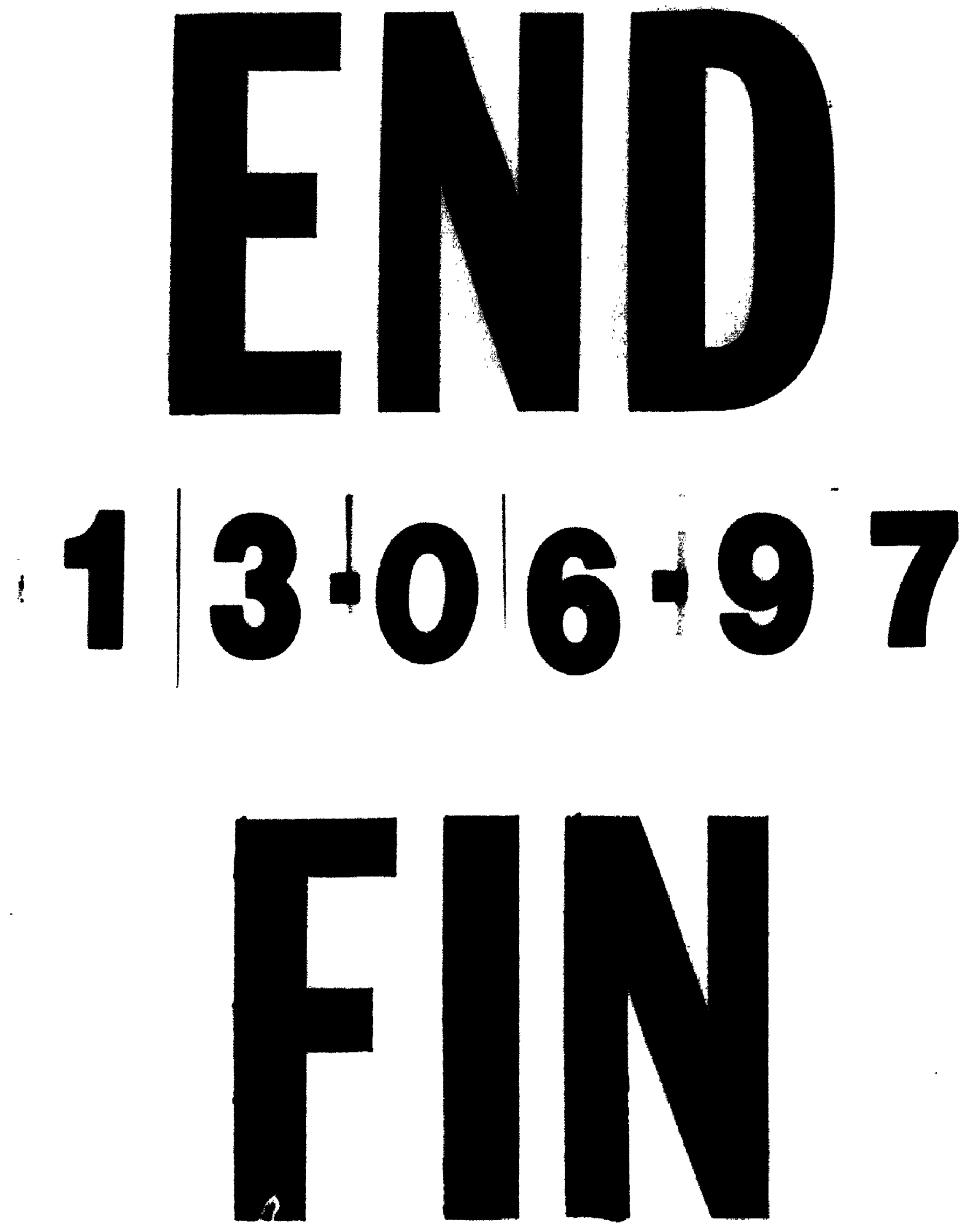